\title{
Action-angle Maps and Scattering Theory for Some Finite-dimensional Integrable Systems \\ II. Solitons, Antisolitons, and their Bound States
}

By

Simon N.M. RuIJSENAARs*

\begin{abstract}
We present an explicit construction of an action-angle map for the nonrelativistic Calogero-Moser systems with $1 / \mathrm{sh}^{2}$ and $-1 / \mathrm{ch}^{2}$ pair potentials, and for relativistic generalizations thereof. The map is used to obtain extensive information concerning dynamics and scattering. We also discuss the relation between the relativistic $N$-particle systems and the $N$-particle-like solutions of various soliton PDEs, including the sine-Gordon equation.
\end{abstract}

\section{Contents}

1. Introduction

2. The direct and inverse transforms
2A. First steps
2B. The case $\tilde{I I}_{n r}$
2C. The case $\tilde{I}_{\text {rel }}$

3. Holomorphy, canonicity and monodromy
3A. The case $\tilde{\mathrm{II}}_{\mathrm{nr}}$
3B. The case $\tilde{\mathrm{II}}_{\mathrm{rel}}$

4. The action-angle transform

4A. The case $\tilde{\mathrm{I}}_{\mathrm{nr}}$

4B. The case $\tilde{\mathrm{I}}_{\mathrm{rel}}$

5. The harmonic oscillator transform

Communicated by H. Araki, February 8, 1993.

1991 Mathematics Subject Classifications: 58F07

* Centre for Mathematics and Computer Science, P.O. Box 4079, 1009

AB Amsterdam, The Netherlands

Work supported by the Netherlands Organisation for the Advancement of Research (NWO). 

5A. The case $\tilde{I}_{n r}$
5B. The case $\tilde{\mathrm{II}}_{\mathrm{rel}}$

6. Dynamics and scattering

6A. The case $\tilde{\mathrm{II}}_{\mathrm{nr}}$

6B. The case $\tilde{\mathrm{I}}_{\text {rel }}(\tau<\pi / 2)$

6C. The case $\tilde{\mathrm{II}}_{\text {rel }}(\tau=\pi / 2)$

7. The relation to soliton solutions revisited

7A. The pure soliton case

7B. Solitons, antisolitons and breathers

Appendix A. Pseudo-self-adjoint and pseudo-unitary matrices

Appendix B. The Cauchy matrix revisited

Appendix C. Spectral asymptotics revisited

References

\section{Introduction}

In this paper we continue our construction and study of action-angle maps initiated in our previous paper [1], which we shall refer to as I. Some of the results reported here have been announced in [2]. A wider context for the results and more background information on the integrable systems at issue (in particular as regards their quantizations) can be found in our survey [3]. This article can be read independently of I, but we do need one key result from I, viz., the canonicity property established there.

The $N$-particle systems studied here can be physically characterized as describing $N_{+}$particles of positive charge (solitons) and $N_{-}$particles of negative charge (antisolitons) in one spatial dimension. From a mathematical viewpoint, we are dealing with integrable systems corresponding to the nonrelativistic Calogero-Moser type Hamiltonian

$$
\begin{aligned}
& H=\frac{1}{2} \sum_{1 \leqslant i \leqslant N_{+}}\left(p_{i}^{+}\right)^{2}+\frac{1}{2} \sum_{1 \leqslant j \leqslant N_{-}}\left(p_{j}^{-}\right)^{2}+\frac{1}{4} g^{2} \mu^{2}\left[\sum_{1 \leqslant i<k \leqslant N_{+}} \frac{1}{\operatorname{sh}^{2} \frac{1}{2} \mu\left(x_{i}^{+}-x_{k}^{+}\right)}\right.
\end{aligned}
$$

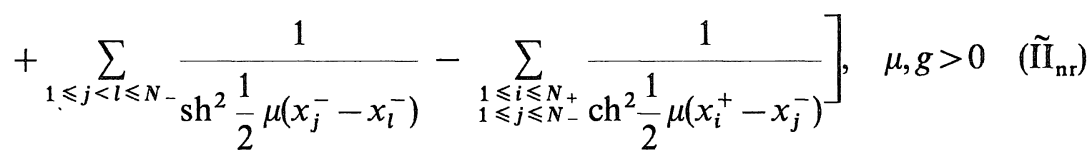


first studied in $[4,5]$, and its relativistic generalization

$$
H=\beta^{-1} \sum_{1 \leqslant i \leqslant N_{+}} \exp \left(\beta p_{i}^{+}\right) V_{i}^{+}+\beta^{-1} \sum_{1 \leqslant j \leqslant N_{-}} \exp \left(\beta p_{j}^{-}\right) V_{j}^{-} \quad\left(\tilde{\mathrm{I}}_{\mathrm{rel}}\right)
$$

introduced in [6]. In (1.2) the potentials and coupling constants are given by

$$
\begin{aligned}
& V_{i}^{+}=\prod_{\substack{1 \leqslant k \leqslant N_{+} \\
k \neq i}}\left[1+\frac{\sin ^{2} \tau}{\operatorname{sh}^{2} \frac{1}{2} \mu\left(x_{i}^{+}-x_{k}^{+}\right)}\right]^{1 / 2} \prod_{1 \leqslant j \leqslant N_{-}}\left[1-\frac{\sin ^{2} \tau}{\operatorname{ch}^{2} \frac{1}{2} \mu\left(x_{i}^{+}-x_{j}^{-}\right.}\right]^{1 / 2} \\
& V_{j}^{-}=\prod_{\substack{1 \leqslant l \leqslant N_{-} \\
l \neq j}}\left[1+\frac{\sin ^{2} \tau}{\operatorname{sh}^{2} \frac{1}{2} \mu\left(x_{j}^{-}-x_{l}^{-}\right)}\right]^{1 / 2} \prod_{1 \leqslant i \leqslant N_{+}}\left[1-\frac{\sin ^{2} \tau}{\operatorname{ch}^{2} \frac{1}{2} \mu\left(x_{j}^{-}-x_{i}^{+}\right)}\right]^{1 / 2} \\
& \tau=\frac{1}{2} \beta \mu g, \quad(\beta, \mu, g) \in(0, \infty)^{3} \cap\{\tau \leqslant \pi / 2\} .
\end{aligned}
$$

The radicands in (1.3) and (1.4) are positive, so we may and will take positive square roots.

In I the special 'pure soliton' case $N_{-}=0$ (or, equivalently, $N_{+}=0$ ) has been dealt with, the systems then being denoted by $\mathrm{II}_{\mathrm{nr}}$ and $\mathrm{II}_{\mathrm{rel}}$. In this paper (in contrast to I) we shall as a rule suppress the dependence on the parameters $\beta$, $\mu$ and $g$, as these are assumed to be fixed and this eases the notation. More generally, our notation is chosen such that many equations for the $\tilde{I}_{n \mathrm{r}}$ case apply to the $\tilde{\mathrm{II}}_{\mathrm{rel}}$ case, too.

For $N_{+} N_{-}>0$ the Hamiltonian (1.2) is not smooth on the hyperplanes $x_{i}^{+}=x_{j}^{-}$when $\tau$ takes its maximum allowed value $\pi / 2$. We shall return to this singular case after presenting some common features of the remaining two cases $\tilde{\mathrm{II}}_{\mathrm{nr}}$ and $\tilde{\mathrm{II}}_{\mathrm{rel}}$ with $\tau \in(0, \pi / 2)$.

First, the phase space will be taken to be the symplectic manifold $\langle\Omega, \omega\rangle$, where

$$
\begin{aligned}
& \Omega \equiv\left\{\left(x^{+}, x^{-}, p^{+}, p^{-}\right) \in R^{2 N} \mid x^{+} \in G_{N_{+}}, x^{-} \in G_{N_{-}}\right\} \\
& \omega \equiv \sum_{1 \leqslant i \leqslant N_{+}} d x_{i}^{+} \wedge d p_{i}^{+}+\sum_{1 \leqslant j \leqslant N_{-}} d x_{j}^{-} \wedge d p_{j}^{-} .
\end{aligned}
$$

Here and below, $G_{n}$ denotes the wedge 


$$
G_{n} \equiv\left\{x \in \boldsymbol{R}^{n} \mid x_{n}<\cdots<x_{1}\right\}
$$

The $N$ independent Hamiltonians in involution can be obtained from an $N \times N$ matrix-valued function $L$ on $\Omega$ specified in Chapter 2, cf. (2.21) and (2.70). A key property of this Lax matrix $L$ is that it is pseudo-self-adjoint w.r.t. the indefinite metric

$$
\mathscr{J} \equiv\left[\begin{array}{cc}
\mathbb{1}_{N_{+}} & 0 \\
0 & -\mathbb{l}_{N_{-}}
\end{array}\right]
$$

Correspondingly, we shall have occasion to use various (known) properties of pseudo-self-adjoint and pseudo-unitary matrices, which are summarized in Appendix A.

Most of our results pertain to the open dense full measure subset of $\Omega$ given by

$$
\Omega_{s} \equiv\{P \in \Omega \mid L(P) \text { has nondegenerate spectrum }\} .
$$

On $\Omega_{s}$ the Lax matrix has $l \in\{0,1, \cdots, m\}$ complex-conjugate pairs of eigenvalues, where

$$
m \equiv \min \left(N_{+}, N_{-}\right)
$$

Such pairs will be shown to correspond to soliton-antisoliton bound states ('breathers'). Any remaining simple eigenvalue $\lambda \in \mathbb{R}$ comes equipped with a signature, viz., the sign of $(\varphi, \mathscr{J} \varphi)$, where $\varphi$ is an eigenvector corresponding to $\lambda$. Specifically, setting

$$
k_{+} \equiv N_{+}-l, \quad k_{-} \equiv N_{-}-l, \quad l=0,1, \cdots, m
$$

there are $k_{+}\left(k_{-}\right)$positive (negative) signature eigenvalues, cf. Appendix A.

For the exceptional case $\tau=\pi / 2$ we take as phase space the toned-down symplectic manifold $\left\langle\Omega^{b}, \omega\right\rangle$, where

$$
\Omega^{b} \equiv\left\{\left(x^{+}, x^{-}, p^{+}, p^{-}\right) \in \Omega \mid x_{i}^{+} \neq x_{j}^{-}, i=1, \cdots, N_{+}, j=1, \cdots, N_{-}\right\} .
$$

With $\Omega_{s}$ replaced by $\Omega_{s}^{b}$ the algebraic features just sketched apply again. Generically, solutions to the Hamilton equations hit the hyperplanes where soliton and antisoliton positions coincide in finite time, so the $H$ flow is not complete on $\Omega^{b}$. However, this disease will be shown to have an effective-if somewhat exotic-cure. 
Having set the stage, we are now prepared to discuss the organization of the paper and its results in more detail. Roughly speaking, the paper consists of three parts. The first part concerns the construction of the action-angle map (Chapters 2-4) and its 'harmonic oscillator' extension (Chapter 5). The second part (Chapter 6) is devoted to a study of the flows generated by the Hamiltonians (1.1), (1.2) and the associated commuting flows. In the third part (Chapter 7) the results obtained in the first two parts are used to study particle-like solutions to various PDEs. The appendices $\mathrm{A}$ and $\mathrm{B}$ contain algebraic results that are used at various places in the paper. Appendix $\mathrm{C}$ deals with spectral asymptotics; it is used only in the second and third part.

The first part of the paper is quite long and involved. To assist the reader in keeping track of the flow chart, a sketch of the main steps - as regards the $\tilde{\mathrm{I}}_{\mathrm{nr}}$ case-now follows. (The treatment of the $\tilde{\mathrm{II}}_{\mathrm{rel}}$ case is quite similar from a conceptual viewpoint.) We shall refer to a number of key equations in Chapters $2-5$, so this sketch might be skipped at first reading and referred back to as needed. (Possibly, this is the best way to proceed as concerns all of the first part.)

Chapter 2 is concerned with the algebraic aspects of the construction of the action-angle map. First of all, this involves a determination of the range of variation of the spectrum of the Lax matrix $L(P)$ as $P$ varies over the subset $\Omega_{l}^{\#}$ of $\Omega_{s}$ where $L$ has $l$ complex-conjugate pairs of eigenvalues, cf. (1.10)-(1.11). A priori, the normal form (A8)-(A12) allows any eigenvalue vector $\lambda$ in the set $\Lambda_{l}$ (2.4). For the concrete Lax matrix (2.21), however, it is not even immediate that $\Omega_{s}$ is non-empty, let alone that $\Omega_{l}^{\#}$ is non-empty for all $l \in\{0,1, \cdots m\}$.

Our strategy is to first obtain restrictions on the set $\Lambda_{l}$ for the pseudo-selfadjoint matrices at hand, and then to prove that any eigenvalue vector in the restricted set actually occurs. The key to this restriction is the implication (2.18), which says that certain auxiliary functions are non-negative wherever a complex-conjugate pair of eigenvalues occurs. We have isolated this implication in Section $2 \mathrm{~A}$ before fully specifying the objects in the fundamental commutation relation (2.7), so as to handle the cases $\tilde{\Pi}_{\mathrm{nr}}$ and $\tilde{\Pi}_{\mathrm{rel}}$ simultaneously and without clutter.

Specializing to the $\widetilde{I}_{n r}$ case in Section $2 \mathrm{~B}$, it is an immediate consequence of the implication (2.18) and the transformed commutation relation (2.24) that the spectrum of the Lax matrix belongs to the closed strip (2.26). Until Chapter 5, however, attention is restricted to the subset $\Omega_{l}$ of $\Omega_{l}^{\#}$ on which the spectrum belongs to the interior of the strip. (The strip boundary corresponds to collapsing tori; such points are dealt with in Section 5A.) Thus, the range of variation $A_{l}$ (2.43) for the eigenvalue vector $\lambda$ arises. 
Via the auxiliary vector-valued function $e$ (given by (2.20)) and a suitable transformation of the commutation relation (2.7) we also associate a vector $v$ of 'norming constants' (2.40) to any point in $\Omega_{l}$. This vector is shown to vary over the set $N_{l}$ (2.44). (In Chapter 4 it will be made clear how $\lambda$ and $v$ determine the actions and angles, resp.; the reparametrizations involved would amount to distracting fuss in the algebraic context of Chapter 2.) In analogy with soliton theory, we have dubbed the transformation from $P \in \Omega_{l}$ to $(v, \lambda) \in N_{l} \times A_{l}$ the 'direct transform'.

The crux is now, that the reasoning can be 'run backwards'. That is, we can start from a point in $N_{l} \times A_{l}$, define matrices and vectors that satisfy the transformed commutation relation, and transform back to the form these objects take on $\Omega_{l}$. In this way it follows not only that $\Omega_{l}$ is non-empty, but also that the direct transform is a bijection from $\Omega_{l}$ onto the data set $D_{l} \equiv N_{l} \times A_{l}$.

The next problem consists in showing that the data set can be promoted to a symplectic manifold in such a way that the (reparametrized) direct transform is a symplectic diffeomorphism. Chapter 3 is a crucial step towards solving this problem: it enables us to reduce the problem to the special (pure soliton) case $N_{+} N_{-}=0$ already handled in I. Specifically, the idea is to exploit the canonicity and analytic features of the diagonalizing map $\Phi$ from $\mathbb{I}$.

Unfortunately, it turns out to be quite difficult to rigorously control the analytic continuation of this map from its definition domain $G_{N} \times \mathbb{R}^{N} \subset \boldsymbol{R}^{2 N}$ to the subsets of $C^{2 N}$ that correspond to the phase spaces (1.6) with $N_{+} N_{-}>0$. Therefore, we have opted for the simpler strategy of controlling the continuation of the inverse map $\mathscr{E}:$ in that case one can block part of the prolific branching that occurs by restricting attention to the domain (3.2). (The excluded branch varieties occur at the boundaries of the strip in (3.2).) The remaining branching can then be determined by estimating the relevant quantities along suitably chosen non-contractible loops, cf. Lemma 3.3.

In Chapter 4 the algebraic and analytic information assembled in Chapters 2 and 3 is combined with geometric information to solve the above problem. As already alluded to, this involves first of all a reparametrization of the data set $D_{l}$, cf. (4.1)-(4.4). The resulting set $\hat{\Omega}_{l}$ (4.5)-(4.7) can then be viewed as a symplectic manifold that arises from quotienting out a free symplectic action of the symmetric group $S_{l}$ on an open subset of a cotangent bundle, equipped with its obvious symplectic form.

The main result of Section 4A (Theorem 4.1) is now, that the reparametrized direct transform $\Phi_{l}$ is indeed a symplectomorphism from $\left\langle\Omega_{l}, \omega\right\rangle$ onto the quotient symplectic manifold $\left\langle\hat{\Omega}_{l}, \hat{\omega}_{l}\right\rangle$. In particular, this reveals that breathers 
cannot be distinguished, a property that is far from obvious a priori-and quite a surprise at the classical level. With hindsight, one may attribute this indistinguishability property to the solitons and antisolitons, too; this viewpoint is suggested especially by Theorem 6.3. But in contrast to the breathers, this picture of the (anti)solitons is not forced by the geometric state of affairs, since the relevant symmetric group action admits a fundamental set that is a manifold. As will be seen, this difference comes down to the fact that distinct real numbers - as opposed to complex ones-admit a continuous ordering.

The commutative diagram (4.19) encompasses a great deal of the notations and constructions of Chapters 2-4. Together with its pure soliton specialization (3.28), it can serve as a blueprint and memory aid. It is also used as a starting point for obtaining the main result of Section 5A, namely Theorem 5.1. Here we show that the action-angle map $\Phi_{l}$ extends to a symplectomorphism $\Phi_{l}^{\#}$ from $\left\langle\Omega_{l}^{\#}, \omega\right\rangle$ onto the symplectic manifold $\left\langle\hat{\Omega}_{l}^{\#}, \hat{\omega}_{l}^{\#}\right\rangle$ defined by (5.15)-(5.19). In new coordinates $u_{i}, v_{i}$ (defined via (5.3)) on the breather part $B_{l}$ of $\hat{\Omega}_{l}$ (cf. (4.5)-(4.7)), the extension amounts to allowing the origins $\left(u_{i}, v_{i}\right)=(0,0), i=1, \cdots, l$.

More specifically, when a pair of complex-conjugate eigenvalues of $L$ converges to the strip boundary, the radius $\left(u_{i}^{2}+v_{i}^{2}\right)^{1 / 2}$ of the corresponding torus goes to 0 . Now this much is plain from the transformation (5.3), but the main problem in proving Theorem 5.1 is to show that arbitrary spectral boundary points do occur and that the extended map remains bijective. The first difficulty is handled by showing that the inverse $\mathscr{E}_{l}$ of $\Phi_{l}$ extends to $\hat{\Omega}_{l}^{\sharp}$, which involves analytic and geometric arguments. In order to obviate the second one, we have to repeat a part of the algebraic reasoning of Section 2B, cf. (5.35)-(5.49). The point is, that a different gauge fixing is needed to control spectral boundary points. (The gauge freedom resides in the $G L(N, C)$ matrix diagonalizing $L$ on $\Omega_{s}$. Thus, the gauge group involved here is a semi-direct product of the $C^{* N}$-valued functions on $\Omega_{s}$ and the symmetric group $S_{N}$, the action of $S_{N}$ being the obvious one.)

The special case $N_{+}=N_{-}=1$ is spelled out in considerable detail at the beginning of Section 5A, cf. (5.1)-(5.12). This should serve as a guide for understanding the notation and the 'harmonic oscillator' terminology associated with the extension procedure for the general case. In Section 5A we also show that the Lax matrix is not diagonalizable on the exceptional set (separatrix) $\Omega_{e} \equiv \Omega \backslash \Omega_{s}$, and present explicit examples of phenomena occurring on $\Omega_{e}$.

After this rather detailed summary of the first part of the paper we now turn to a shorter description of the results in the second part, Chapter 6 . Here, the extensive information gathered in the first part is used to study the character 
and temporal asymptotics of a large class of mutually commuting Hamiltonian flows, containing in particular the flows generated by the Hamiltonians (1.1) and (1.2) in the nonrelativistic and relativistic case, resp. Just as in the pure soliton regime, the position part $\left(x^{+}(t), x^{-}(t)\right)$ of the flows can be expressed in terms of the eigenvalues of an explicitly known $t$-dependent $N \times N$ matrix. Therefore, studying scattering theory is again tantamount to studying spectral asymptotics.

In the pure soliton case we could determine the desired spectral asymptotics for matrices defined on open neighborhoods of phase space viewed as a subset of $C^{2 N}$. This led to uniform estimates without which the canonicity proof in I would break down. Here however, venturing into complex neighborhoods is troublesome, as this leads to breather dissociation.

However, since we solve the canonicity problem via analytic continuation, we only need the spectral asymptotics for the type of matrices arising on phase space. This is dealt with in Appendix $\mathrm{C}$ in a slightly more general setting. The main result, Theorem $\mathrm{C} 1$, is of independent interest. The reality restriction (as compared to Appendix A in I) enables us to obtain quite precise information on the times needed for eigenvalue clusters to collide and separate, and on the behavior before collision and after separation. For the matrices occurring in Chapter 6 the shifts of the center of mass of each cluster (and of the phase in case the cluster consists of one breather) can be calculated explicitly by exploiting an explicit formula for the inverse of the Cauchy matrix, cf. Appendix B. From a physical point of view, the corresonding elucidation of the long-time asymptotics of the various Hamiltonian flows (to be found in Theorems 6.2, 6.5 and 6.8) may be regarded as the principal result of this paper.

Besides the general case, Chapter 6 also deals with a special situation of considerable physical interest. It so happens that the collapse of the invariant tori already discussed above corresponds physically to breathers that do not breathe any more. In the special case $N_{+}=N_{-} \equiv M$ there exists an invariant submanifold of phase space characterized by $x^{+}=x^{-}, p^{+}=p^{-}$, which corresponds to $M$ such breathers. We prove that the action-angle map restricted to this submanifold coincides with the action-angle map for $M$ solitons up to rescalings, cf. Theorems 6.3, 6.6. Pictorially speaking, this says that dead breathers behave like solitons. (For the $\mathrm{II}_{\mathrm{nr}}$ case and the $H$ flow this phenomenon was first observed by Calogero [7].)

Another striking phenomenon pertaining to the singular $\tau$-value $\pi / 2$ is the existence of simultaneous collisions of all solitons and antisolitons. This can only happen when the asymptotic soliton and antisoliton momenta are interlaced, so that one must have $\left|N_{+}-N_{-}\right| \leqslant 1$. This restriction is quite surpris- 
ing; as a bonus, the existence proof corroborates the inevitability of somewhat complicated signs in several formulas occurring below.

Our results for the $\tilde{\mathrm{II}}_{\mathrm{nr}}$ and $\tilde{\mathrm{II}}_{\mathrm{rel}}(\tau<\pi / 2)$ systems are presented in Sections $6 \mathrm{~A}$ and $6 \mathrm{~B}$, resp. These are quite complete; the problems left open concern the exceptional set $\Omega \backslash \Omega_{s}$. In contrast, our account of the $\tilde{\mathrm{II}}_{\text {rel }}(\tau=\pi / 2)$ case in Section $6 \mathrm{C}$ leads to some questions that are left unanswered even for $\Omega_{s}^{b}$. These questions are connected with the fact that the commuting local flows on $\Omega^{b}$ are not global: Even though we have found a way to complete the flows on a jazzed-up phase space $\Omega^{\#}$, one winds up with some problems that appear quite elusive.

In order to discuss Chapter 7, we begin by commenting on our terminology 'solitons' and 'antisolitons', as applied to the point particles and antiparticles of the $\tilde{\mathrm{I}}_{\mathrm{nr}}$ and $\tilde{\mathrm{I}}_{\mathrm{rel}}$ systems. This terminology is natural first of all because the factorization of the $S$-map revealed in Chapter 6 is the hallmark of the scattering of the soliton-antisoliton solutions of the sine-Gordon and modified Kortewegde Vries equations. These solutions have been viewed as 'particle-like' ever since they were discovered some 20 years ago. However, the fact that the particle character of soliton solutions to various PDEs and infinite lattices (such as the Toda lattice) can be understood from an intimate relation to integrable finitedimensional Hamiltonian systems (viz., the $\mathrm{II}_{\text {rel }}$ systems) has only been demonstrated in recent years $[6,2,3]$.

We mention in passing that this relation has an intriguing resemblance to phenomena in the area of non-integrable PDEs, such as the Navier-Stokes equation [8]. Note in this connection that in actual applications of soliton PDEs the relevant PDE is typically obtained after some drastic approximations of a more accurate, but non-integrable description; in many such cases, the observed long-time behavior is indeed finite-dimensional (namely, 'solitonic').

In the first section of Chapter 7 we reconsider the particle-soliton relation, firstly with regard to the notion of soliton space-time trajectory $[6,2]$ and secondly with the aim of proving uniform decay bounds of the form

$$
\sup _{y \in \boldsymbol{R}}\left|\psi(t, y)-\psi^{ \pm}(t, y)\right|=O(\exp (\mp t r)), \quad t \rightarrow \pm \infty
$$

Here, $\psi(t, y)$ denotes an $N$-soliton solution and $\psi^{ \pm}(t, y)$ denote linear combinations of $N$ 1-soliton solutions; the constant $r>0$ can be expressed in terms of the action variables associated with the initial point in phase space that determines the Cauchy initial value of the soliton evolution equation. 
As it turns out, the correspondence between particles and solitons, combined with Appendix C, can be used to great advantage to prove (1.14). For the pure soliton solutions of the $\mathrm{KdV}$ and modified $\mathrm{KdV}$ equations these bounds improve previous results of Tanaka [9] and Ohmiya [10], resp., who proved that the lhs of $(1.14)_{ \pm}$converges to 0 for $t \rightarrow \pm \infty$. It is easy to check that our decay estimates cannot be sharpened for $N=2$, and we believe that they are best possible for general $N$, too.

In Section 7A we make essential use of (a special case of Theorem C1, but the previous chapters are only needed in as much as they subsume the pure soliton case already handled in $\mathbb{I}$. In contrast, Section 7B leans heavily on Chapters 2-6. Here, we use virtually all of our results on the $\tau=\pi / 2 \tilde{\mathrm{I}}_{\text {rel }}$ systems to study a class of sine-Gordon and modified $\mathrm{KdV}$ solutions. This class subsumes all real-valued Schwartz space solutions that yield a vanishing reflection coefficient and a transmission coefficient having $N$ poles in the upper half plane, each of which is simple. (Here and below, we use terminology associated with the Inverse Scattering Transform (IST), cf. e.g. [11, 12].)

We first generalize the bounds $(1.14)_{ \pm}$to those solutions that contain solitons, antisolitons and breathers with distinct asymptotic velocities. We then detail the link with the particle systems and prove that any solution in our class is reflectionless in the sense of the IST. Finally, after discussing the relation of the exceptional set $\Omega^{b} \backslash \Omega_{s}^{b}$ and multipole solutions [13-15], we extend the notion of space-time trajectories to any solution in our class and derive various properties of these trajectories. The occurrence of soliton-antisoliton and multiple collisions gives rise to a far richer picture than in the pure soliton case.

In Chapter 7 we also comment on related literature [2, 3, 9-17].

We conclude this Introduction with some general remarks concerning organisation and presentation. A superficial reading of this paper might give an impression of a certain redundancy, both in the notation and in the number of gauge fixings and permutations employed. Now we do not presume that the exposition cannot be improved, but we do believe that any clear-cut and rigorous account of the results occurring below will have a quite baroque appearance.

The strategy followed here has the advantage of clearly distinguishing the algebraic, analytic and geometric problems involved, at the expense of a small amount of repetition. In our choice and use of gauge-dependent objects we have attempted to obtain maximal information with minimal notation for each of the three aspects before putting the pieces together. The various permutations are a trifling nuisance from a conceptual point of view, but their use appears inevitable in establishing unambiguous notation and definitions. We should 
also mention that a rigorous grip on what we have dubbed the harmonic oscillator transform seems unattainable without first getting the action-angle map under control.

Finally, we are aware of results in abstract symplectic geometry (described e.g. in [18]) that may seem to have a bearing on some of the problems encountered below, but these results hinge on certain compactness assumptions. Our sole hypotheses are (1.1)-(1.5), and it so happens that these equations give rise to concrete integrable systems for which these compactness assumptions are violated. In fact, for better or worse we use very little geometric machinery and, more generally, this paper is largely self-contained.

\section{The Direct and Inverse Transforms}

\section{A. First Steps}

In this section we lay the groundwork for the construction of the direct transform for both cases at once, without detailing the Lax matrix $L$ yet. First, fixing a point $P$ in the subset $\Omega_{s}$ on which $L$ has simple spectrum, we may invoke Prop. A3 to conclude that a $\mathscr{J}$-unitary $U$ exists such that $U^{-1} L U$ takes the normal form (A8)-(A12). (For the critical value $\tau=\pi / 2$ we take $P \in \Omega_{s}^{b}$.) Setting

$$
K \equiv \operatorname{diag}\left(\mathbf{1}_{k_{+}}, 2^{-1 / 2}\left[\begin{array}{cc}
\mathbf{1}_{l} & \mathbf{1}_{l} \\
\mathbf{1}_{l} & -\mathbf{1}_{l}
\end{array}\right], \quad \mathbf{1}_{k_{-}}\right)=K^{-1}=K^{*}
$$

and

$$
\hat{L} \equiv K U^{-1} L U K
$$

we then obtain

$$
\hat{L}=\operatorname{diag}\left(p_{1}, \cdots, p_{k_{+}}, \rho_{1}+i \sigma_{1}, \cdots, \rho_{l}+i \sigma_{l}, \rho_{1}-i \sigma_{1}, \cdots, \rho_{l}-i \sigma_{l}, n_{1}, \cdots, n_{k_{-}}\right) .
$$

Since the positive and negative signature eigenvalues $p_{i}$ and $n_{j}$ are distinct, we may and will require that they are ordered such that $p \in G_{k_{+}}, n \in G_{k_{-}}$(recall (1.8)). Similarly, we may and will order $\rho_{1}+i \sigma_{1}, \cdots, \rho_{l}+i \sigma_{l}$ lexicographically; Namely, we require $\rho_{l} \leqslant \cdots \leqslant \rho_{1}$, and if $\rho_{k+1}=\rho_{k}$, then we require $\sigma_{k+1}<\sigma_{k}$.

With these ordering conventions in force from now on, we introduce a vector $\lambda$ in the set

$$
\Lambda_{l} \equiv\left\{\lambda \in C^{N} \mid\left(\lambda_{1}, \cdots, \lambda_{k_{+}}\right) \in G_{k_{+}},\left(\lambda_{N-k_{-}+1}, \cdots, \lambda_{N}\right) \in G_{k_{-}},\right.
$$




$$
\begin{aligned}
& \lambda_{k_{+}+l+n}=\bar{\lambda}_{k_{+}+n}, \operatorname{Im} \lambda_{k_{+}+n}>0, n=1, \cdots, l, \\
& \left.\lambda_{j} \neq \lambda_{k} \text { when } j \neq k, \lambda_{k_{+}+1}, \cdots, \lambda_{k_{+}+} \text {in lexicographic order }\right\}
\end{aligned}
$$

by writing $\hat{L}$ as

$$
\hat{L}=\operatorname{diag}\left(\lambda_{1}, \cdots, \lambda_{N}\right)
$$

(In (2.4) and below, bars denote complex conjugation.) A priori, any $\lambda \in \Lambda_{l}$ might arise. However, this is not the case for the matrices $L$ specified below. Just as in I, a key tool to obtain detailed spectral information is a commutation relation involving $L$ and a matrix-valued function $A$ on $\Omega$,

$$
A \equiv \operatorname{diag}\left(\exp \left(\mu x_{1}^{+}\right), \cdots, \exp \left(\mu x_{N_{+}}^{+}\right),-\exp \left(\mu x_{1}^{-}\right), \cdots,-\exp \left(\mu x_{N_{-}}^{-}\right)\right)
$$

This commutation relation takes the form

$$
c[A, L]=e \otimes e- \begin{cases}A & (\mathrm{nr}) \\ (A L+L A) / 2 & (\mathrm{rel})\end{cases}
$$

where the constant $c$ and vector $e$ will be detailed below (cf. (2.19), (2.20) and (2.68), (2.69)). Setting

$$
\begin{gathered}
\hat{A} \equiv K U^{-1} A U K \\
\hat{e} \equiv K U^{-1} e, \quad \tilde{e} \equiv K U^{t} e
\end{gathered}
$$

we may transform (2.7) to get

$$
c[\hat{A}, \hat{L}]=\hat{e} \otimes \tilde{e}-\left\{\begin{array}{lc}
\hat{A} & (\mathrm{nr}) \\
(\hat{A} \hat{L}+\hat{L} \hat{A}) / 2 & (\mathrm{rel}) .
\end{array}\right.
$$

Next, we derive an implication ((2.18) below), which can be combined with (2.10) to obtain information on the eigenvalue vector $\lambda$. First, we note that

$$
A \mathscr{J}>0
$$

cf. the definitions (2.6) and (1.9). This entails

where

$$
\hat{A} \hat{\mathscr{J}}>0 \text {, }
$$




$$
\hat{\mathscr{J}} \equiv K \mathscr{J} K=\operatorname{diag}\left(\mathbb{1}_{k_{+}}, \quad\left[\begin{array}{ll}
0 & \mathbb{1}_{l} \\
\mathbf{1}_{l} & 0
\end{array}\right],-\mathbb{1}_{k_{-}}\right),
$$

since we may rewrite $\hat{A} \hat{\mathscr{J}}$ as

$$
\hat{A} \hat{\mathscr{J}}=K U^{-1} A U \mathscr{J} K=\left(K U^{-1}\right)(A \mathscr{J})\left(K U^{-1}\right)^{*} .
$$

Second, in both cases it can be read off from the definition of $e$ (cf. (2.20), (2.69)) that

$$
\bar{e}=\mathscr{J} e
$$

This implies

$$
\tilde{e}=\hat{\mathscr{J}} \overline{\hat{e}}
$$

since we may write

$$
\tilde{e}=K U^{t} e=K \mathscr{J} \bar{U}^{-1} \mathscr{J} e=(K \mathscr{J} K)\left(\overline{K U^{-1}}\right) \bar{e} .
$$

Now let $\lambda_{j}$ be a non-real eigenvalue and let $k$ be the index such that $\lambda_{k}=\bar{\lambda}_{j}$. Due to (2.12) we have $(\hat{A} \hat{J})_{j j},(\hat{A} \hat{J})_{k k}>0$; also, combining (2.16) and (2.13) we infer $\tilde{e}_{j}=\overline{\hat{e}}_{k}, \tilde{e}_{k}=\hat{e}_{j}$. Therefore, the following implication holds true:

$$
\lambda_{j}=\rho_{n} \pm i \sigma_{n}, \lambda_{k}=\rho_{n} \mp i \sigma_{n} \Rightarrow \hat{A}_{j k}>0, \hat{e}_{j} \tilde{e}_{k} \geqslant 0 .
$$

At this point it is expedient to proceed with a separate treatment of both cases, detailing first $c, e$ and $L$. However, it should be noted that these quantities are in essence uniquely determined by the commutation relation (2.7) and the definition (2.6) of $A$, once one insists on $c \neq 0, L$ being $\mathscr{J}$-s.a. and $e$ satisfying (2.15).

\section{B. The Case $\tilde{\Pi}_{n r}$}

In the nonrelativistic case $c, e$ and $L$ are explicitly given by

$$
\begin{aligned}
& c \equiv-i / \mu g \quad \mu, g \in(0, \infty) \\
& e_{i} \equiv \exp \left(\frac{1}{2} \mu x_{i}^{+}\right), \quad e_{N_{+}+j} \equiv i \exp \left(\frac{1}{2} \mu x_{j}^{-}\right) \\
& L_{i k} \equiv \delta_{i k} p_{i}^{+}+\left(1-\delta_{i k}\right) \frac{i \mu g}{2 \operatorname{sh} \frac{1}{2} \mu\left(x_{i}^{+}-x_{k}^{+}\right)} \quad L_{i, N_{+}+l} \equiv \frac{-\mu g}{2 \operatorname{ch} \frac{1}{2} \mu\left(x_{i}^{+}-x_{l}^{-}\right)}
\end{aligned}
$$




$$
L_{N_{+}+j, k} \equiv \frac{\mu g}{2 \operatorname{ch} \frac{1}{2} \mu\left(x_{j}^{-}-x_{k}^{+}\right)} \quad L_{N_{+}+j, N_{+}+l} \equiv \delta_{j l} p_{j}^{-}+\left(1-\delta_{j l}\right) \frac{i \mu g}{2 \operatorname{sh} \frac{1}{2} \mu\left(x_{j}^{-}-x_{l}^{-}\right)}
$$

where $i, k \in\left\{1, \cdots, N_{+}\right\}$and $j, l \in\left\{1, \cdots, N_{-}\right\}$. Since $\mu$ and $g$ are positive, $L$ is of the form

$$
L=\left[\begin{array}{ll}
L_{++} & L_{+-} \\
L_{-+} & L_{--}
\end{array}\right], \quad L_{\delta \delta}^{*}=L_{\delta \delta}, L_{\delta,-\delta} *=-L_{-\delta, \delta}, \quad \delta=+,-
$$

so that $L$ is $\mathscr{J}$-s.a., as announced. Furthermore, the equality (2.15) is evident and the commutation relation (2.7) is easily verified. For later use we note the crucial relation

$$
H=\frac{1}{2} \operatorname{Tr} L^{2}
$$

cf. (1.1).

We now continue the construction of the direct transform begun in Section 2A. Rewriting the commutation relation (2.10) we obtain

$$
\hat{A}_{j k}\left[1+i\left(\lambda_{j}-\lambda_{k}\right) / \mu g\right]=\hat{e}_{j} \tilde{e}_{k}
$$

Combining this with the implication (2.18), we infer

$$
1 \mp 2 \sigma_{n} / \mu g \geqslant 0, \quad n=1, \cdots, l .
$$

But then we may conclude

$$
\sigma(L) \subset S_{\mu g / 2}^{c l}
$$

where $S_{w}$ denotes a strip defined by

$$
S_{w} \equiv\{\lambda \in C \| \operatorname{Im} \lambda \mid<w\}
$$

and where $c l$ denotes closure.

Next, we introduce the subsets

$$
\Omega_{i} \equiv\left\{P \in \Omega \mid \sigma(L(P)) \subset S_{\mu g / 2}\right\}
$$




$$
\begin{aligned}
& \Omega_{b} \equiv \Omega \backslash \Omega_{i} \\
& \Omega_{r} \equiv \Omega_{i} \cap \Omega_{s}
\end{aligned}
$$

(where $i, b, r$ stand for interior, boundary, regular). From (2.24) and (2.16) we now conclude

$$
\lambda_{j}=\rho_{n}+\frac{1}{2} i \mu g, \quad \lambda_{k}=\rho_{n}-\frac{1}{2} i \mu g \Rightarrow \hat{e}_{j}=\tilde{e}_{k}=0
$$

In contrast, we claim that

$$
\hat{e}_{j} \neq 0, \quad j=1, \cdots, N, \quad\left(\text { on } \Omega_{r}\right) .
$$

Indeed, the square brackets in (2.24) are non-zero on $\Omega_{r}$, so we may rewrite (2.24) as

$$
\hat{A}_{j k}=\hat{e}_{j} C(0, \mu,-g ; \lambda, \lambda)_{j k} \tilde{e}_{k}, \quad\left(\text { on } \Omega_{r}\right)
$$

where $C$ is the Cauchy matrix (B1). Now we clearly have $|\hat{A}|=|A| \neq 0$, cf. (2.6); moreover, $|C| \neq 0$ in view of Cauchy's identity (B4). Therefore, our claim (2.32) follows.

We proceed by introducing

$$
E \equiv \operatorname{diag}\left(\hat{e}_{1}, \cdots, \hat{e}_{N}\right)
$$

Restricting attention to $\Omega_{r}$ from now on, we see from (2.32) that $E$ is invertible. Thus we may define the product matrix

$$
\mathscr{P} \equiv E^{-1} K U^{-1}
$$

and then we get from (2.2), (2.5) and (2.9) the relations

$$
\begin{aligned}
& \mathscr{P} L^{P}{ }^{-1}=\operatorname{diag}\left(\lambda_{1}, \cdots, \lambda_{N}\right) \\
& \mathscr{P}_{e}=(1, \cdots, 1) \equiv \zeta
\end{aligned}
$$

We also introduce

$$
\begin{aligned}
& v \equiv \mathscr{P}^{-1 t} e \\
& \tilde{A} \equiv \mathscr{P} A \mathscr{P}^{-1} .
\end{aligned}
$$

Then we have from (2.9) 


$$
v_{k}=\hat{e}_{k} \tilde{e}_{k}, \quad k=1, \cdots, N
$$

and from (2.33)

$$
\tilde{A}_{j k}=C(0, \mu,-g ; \lambda, \lambda)_{j k} v_{k}
$$

Finally, recalling (2.16) and (2.13), we conclude from (2.40) and (2.32):

$$
\begin{array}{ll}
v_{i} \in(0, \infty), & i=1, \cdots, k_{+} \\
v_{N-k_{-}+j} \in(-\infty, 0), & j=1, \cdots, k_{-} \\
v_{k_{+}+l+n}=\bar{v}_{k_{+}+n} \in \mathbb{C}^{*}, & n=1, \cdots, l .
\end{array}
$$

Let us now take stock of our findings. We have associated to any point in $\Omega_{r}$ for which $L$ has $2 l$ (and only $2 l$ ) non-real eigenvalues the following data: An eigenvalue vector in the set

$$
A_{l} \equiv \Lambda_{l} \cap\left(S_{\mu g / 2}\right)^{N}
$$

(recall (2.4), (2.27)), and a vector $v$ of 'norming constants' in the set

$$
\begin{aligned}
& N_{l} \equiv\left\{v \in \mathbb{C}^{N} \mid v_{i} \in(0, \infty), \quad i=1, \cdots, k_{+}\right. \\
&\left.v_{N-k_{-}+j} \in(-\infty, 0), \quad j=1, \cdots, k_{-}, \quad v_{k_{+}+l+n}=\bar{v}_{k_{+}+n} \in \mathbb{C}^{*}, \quad n=1, \cdots, l\right\} .
\end{aligned}
$$

Moreover, the product matrix $\mathscr{P}$ used to define the association is uniquely determined by (2.36) and (2.37) (in contrast to its factors). Indeed, the eigenvalues $\lambda_{1}, \cdots, \lambda_{N}$ are distinct and ordered, so that (2.36) determines $\mathscr{P}$ up to left multiplication by a regular diagonal matrix, and then (2.37) fixes this gauge ambiguity.

The upshot is, therefore, that we obtain a well-defined direct transform

$$
\mathscr{D}: \Omega_{r} \rightarrow D, \quad\left(x^{+}, x^{-}, p^{+}, p^{-}\right) \mapsto(v, a), \quad a \equiv \lambda
$$

where the data set $D$ is given by

$$
D \equiv \bigcup_{l=0}^{m} D_{l}, \quad D_{l} \equiv N_{l} \times A_{l}
$$

At this point a comment on the change of notation $\lambda \rightarrow a$ in (2.45) (and below) is in order: We are anticipating the $\tilde{\Pi}_{r e l}$ case, where the action vector $a$ is not 
equal to the eigenvalue vector $\lambda$. Various formulas involving this symbol will apply without change to the $\tilde{\mathrm{II}}_{\mathrm{rel}}$ case because of this change in notation.

We continue by proving that $\mathscr{D}$ is a bijection. To this end, we choose a point $(v, a) \in D_{l}$ and define the matrices

$$
\begin{aligned}
& \tilde{A}(v, a)_{j k} \equiv C(0, \mu,-g ; a, a)_{j k} v_{k} \\
& \tilde{L}(a) \equiv \operatorname{diag}\left(a_{1}, \cdots, a_{N}\right) .
\end{aligned}
$$

Then the commutation relation

$$
c[\tilde{A}, \tilde{L}]=\zeta \otimes v-\tilde{A}
$$

is easily verified. Next, we introduce

$$
\begin{aligned}
& s_{i} \equiv v_{i}^{1 / 2}, \quad i=1, \cdots, k_{+} \\
& s_{i} \equiv\left\{\begin{array}{l}
v_{i} \\
1
\end{array}, \quad i=\left\{\begin{array}{l}
k_{+}+1, \cdots, k_{+}+l \\
k_{+}+l+1, \cdots, k_{+}+2 l
\end{array}\right.\right. \\
& s_{i} \equiv\left(-v_{i}\right)^{1 / 2}, \quad i=N-k_{-}+1, \cdots, N \\
& S \equiv \operatorname{diag}\left(s_{1}, \cdots, s_{N}\right),
\end{aligned}
$$

make a similarity transformation

$$
A_{s} \equiv S \tilde{A} S^{-1}
$$

and consider the matrix $A_{s} \hat{\mathscr{J}}$. Using the definition (2.13) of $\hat{\mathscr{J}}$ and the properties of the numbers $a_{k}, v_{k}$ and $s_{k}$, it readily follows that this matrix can be written

$$
\left(A_{s} \hat{\mathscr{J}}\right)_{j k}=s_{j} C(0, \mu,-g ; a, \bar{a})_{j k} \bar{s}_{k} .
$$

Let us now come to the point of this: The Cauchy matrix occurring in (2.55) is positive by virtue of Lemma B1. But then it is clear from (2.55) that $A_{s} \hat{\mathscr{J}}$ is positive, too. Therefore, $A_{s}$ is $\hat{\mathscr{J}}$-s.a. and we may invoke Prop. A1 to conclude that $A_{s}$ is diagonalizable and that $A_{s}$ has $N_{+}$positive and $N_{-}$negative eigenvalues.

As a result, we may conclude that there exists a regular matrix $\mathscr{M}$ such that

$$
\mathscr{M}^{-1} \tilde{A} \mathscr{M}=\operatorname{diag}\left(\alpha_{1}, \cdots, \alpha_{N}\right)
$$

where 


$$
\alpha_{1}, \cdots, \alpha_{N_{+}} \in(0, \infty), \quad \alpha_{N_{+}+1}, \cdots, \alpha_{N} \in(-\infty, 0)
$$

But now we can exploit the commutation relation (2.49): It entails

$$
\left(\mathscr{M}^{-1} \tilde{L} \mathscr{M}\right)_{j k}\left(\alpha_{j}-\alpha_{k}\right)=i \mu g\left(\left(\mathscr{M}^{-1} \zeta\right)_{j}\left(\mathscr{M}^{t} v\right)_{k}-\delta_{j k} \alpha_{j}\right)
$$

so that

$$
\left(\mathscr{M}^{-1} \zeta\right)_{j}\left(\mathscr{M}^{t} v\right)_{j}=\alpha_{j} \neq 0, \quad j=1, \cdots, N
$$

Hence the $r h s$ of (2.58) is non-zero for $j \neq k$, and so we may deduce that $\tilde{A}$ has simple spectrum. Thus, we may order the eigenvalues in such a way that the definitions

$$
\begin{array}{ll}
x_{i}^{+} \equiv \mu^{-1} \ln \alpha_{i}, & i=1, \cdots, N_{+} \\
x_{j}^{-} \equiv \mu^{-1} \ln \left(-\alpha_{N_{+}+j}\right), & j=1, \cdots, N_{-}
\end{array}
$$

entail

$$
x^{\delta} \in G_{N_{\tilde{\delta}}}, \quad \delta=+,-
$$

Clearly, this fixes $\mathscr{M}$ up to right multiplication by a regular diagonal matrix.

In order to complete the definition of the inverse transform, we now set

$$
\begin{array}{ll}
p_{i}^{+} \equiv\left(\mathscr{M}^{-1} \tilde{L} \mathscr{M}\right)_{i i}, & i=1, \cdots, N_{+} \\
p_{j}^{-} \equiv\left(\mathscr{M}^{-1} \tilde{L} \mathscr{M}\right)_{N_{+}+j, N_{+}+j}, & j=1, \cdots, N_{-} .
\end{array}
$$

We assert that these numbers are real. To prove this, we shall exploit Fact (6) from Appendix A, as follows. We have already shown that $A_{s}$ is $\hat{\mathscr{J}}$-s.a. and that $\tilde{A}$ has simple and real spectrum. Therefore, $K A_{s} K$ is $\mathscr{J}$-s.a. and has simple and real spectrum. Invoking now Prop.A3, we conclude that $K A_{s} K$ can be diagonalized by a $\mathscr{J}$-unitary $V$. The Fact mentioned above then implies that the numbers $\left(V^{-1} K \tilde{L} K V\right)_{k k}, k=1, \cdots, N$, are real. (Note that $K \tilde{L} K$ is $\mathscr{J}$-s.a., so that $V^{-1} K \tilde{L} K V$ is $\mathscr{J}$-s.a., too.) But these real numbers must be a permutation of the numbers $p_{i}^{+}, p_{j}^{-}$, since the matrices $S^{-1} K V$ and $\mathscr{M}$ both diagonalize $\tilde{A}$ and, therefore, are related via right multiplication by a permutation matrix and a diagonal matrix. Hence our assertion follows.

Since the gauge ambiguity in $\mathscr{M}$ does not influence the diagonal elements of $\mathscr{M}^{-1} \tilde{L} \mathscr{M}$, we have now obtained a well-defined map

$$
\mathscr{I}: D \rightarrow \Omega,(v, a) \mapsto\left(x^{+}, x^{-}, p^{+}, p^{-}\right) .
$$


Lemma 2.1. The map $\mathscr{I}$ is a bijection onto $\Omega_{r}$ whose inverse is the map $\mathscr{D}$.

Proof. Since $\left(\mathscr{M}^{-1} \zeta\right)_{j} \neq 0$ due to (2.59), we may and will fix the ambiguity in $\mathscr{M}$ by requiring

$$
\mathscr{M}^{-1} \zeta=e
$$

where $e$ is defined via (2.20) and (2.60), (2.61). Then it follows from (2.59) that

$$
\mathscr{M}^{t} v=e
$$

The point of this gauge choice is, that when we fix $(v, a) \in D$, yielding a point $P \equiv \mathscr{I}(v, a) \in \Omega$, then the matrix $L$ evaluated in $P$ equals $\mathscr{M}^{-1} \tilde{L} \mathscr{M}$. (To verify this, note these two matrices have equal diagonals in view of (2.63), (2.64) and (2.21). Next, combine (2.66), (2.67) with (2.58) to express the off-diagonal elements in terms of $x^{+}, x^{-}$, which yields the off-diagonal elements in (2.21).) Consequently, the numbers $a_{1}, \cdots, a_{N}$ are just the ordered eigenvalues of $L(P)$. Since they are distinct and belong to $S_{\mu g / 2}$ we conclude that $P \in \Omega_{r}$. Thus, $\mathscr{I}$ maps $D$ into $\Omega_{r}$.

Next, we note that the matrix $\mathscr{P}(P)$ must be equal to $\mathscr{M}$. Indeed, by construction $\mathscr{M}$ has the properties (2.36), (2.37) that uniquely determine $\mathscr{P}(P)$, cf. (2.66). But then we may deduce $\mathscr{D} \circ \mathscr{I}=\mathrm{id}_{D}$.

Conversely, picking some $P_{0} \in \Omega_{r}$, the matrix $\mathscr{M}$ evaluated in $\mathscr{D}\left(P_{0}\right)$ must coincide with $\mathscr{P}\left(P_{0}\right)$, again by uniqueness. Thus we also have $\mathscr{I} \circ \mathscr{D}=\mathrm{id}_{\Omega_{r}}$ and the lemma follows.

\section{C. The Case $\tilde{I}_{\text {rel }}$}

In the relativistic case $c, e$ and $L$ are defined by

$$
\begin{aligned}
& c \equiv-\frac{i}{2} \cot \tau, \quad \tau \equiv \frac{1}{2} \beta \mu g \in(0, \pi / 2] \\
& e_{i} \equiv \exp \left(\frac{1}{2} \mu x_{i}^{+}+\frac{1}{2} \beta p_{i}^{+}\right)\left(V_{i}^{+}\right)^{1 / 2}, \quad e_{N_{+}+j} \equiv i \exp \left(\frac{1}{2} \mu x_{j}^{-}+\frac{1}{2} \beta p_{j}^{-}\right)\left(V_{j}^{-}\right)^{1 / 2} \\
& L_{i k} \equiv \exp \left(\frac{1}{2} \beta\left[p_{i}^{+}+p_{k}^{+}\right]\right)\left(V_{i}^{+} V_{k}^{+}\right)^{1 / 2} \frac{i \sin \tau}{\operatorname{sh} \frac{1}{2} \mu\left(x_{i}^{+}-x_{k}^{+}+i \beta g\right)}
\end{aligned}
$$




$$
\begin{aligned}
& L_{N_{+}+j, N_{+}+l} \equiv \exp \left(\frac{1}{2} \beta\left[p_{j}^{-}+p_{l}^{-}\right]\right)\left(V_{j}^{-} V_{l}^{-}\right)^{1 / 2} \frac{i \sin \tau}{\operatorname{sh} \frac{1}{2} \mu\left(x_{j}^{-}-x_{l}^{-}+i \beta g\right)} \\
& L_{N_{+}+j, k} \equiv \exp \left(\frac{1}{2} \beta\left[p_{j}^{-}+p_{k}^{+}\right]\right)\left(V_{j}^{-} V_{k}^{+}\right)^{1 / 2} \frac{\sin \tau}{\operatorname{ch} \frac{1}{2} \mu\left(x_{j}^{-}-x_{k}^{+}+i \beta g\right)} \\
& L_{i, N_{+}+l} \equiv-\bar{L}_{N_{+}+l . i}
\end{aligned}
$$

where $i, k \in\left\{1, \cdots, N_{+}\right\}$and $j, l \in\left\{1, \cdots, N_{-}\right\}$, and where the potentials $V_{i}^{+}, V_{j}^{-}$are defined by (1.3), (1.4). Note that for $\tau=\pi / 2$ the Lax matrix is real-analytic on $\Omega^{b}$, but gets singular on the hyperplanes $x_{i}^{+}=x_{j}^{-}$. Substituting

$$
\beta \leftrightarrow \mu, \quad p, q \rightarrow\left(x_{1}^{+}, \cdots, x_{N_{+}}^{+}, x_{1}^{-}+i \pi / 2 \mu, \cdots, x_{N_{-}}^{-}+i \pi / 2 \mu\right)
$$

in (B1), (B4), one obtains

$$
|L|=\exp \beta\left[\sum_{1 \leqslant i \leqslant N_{+}} p_{i}^{+}+\sum_{1 \leqslant j \leqslant N_{-}} p_{j}^{-}\right]
$$

on $\Omega$ for $\tau \in(0, \pi / 2)$ and on $\Omega^{b}$ for $\tau=\pi / 2$. Again, $L$ is of the form (2.22) and hence is $\mathscr{J}$-s.a. Also, $(2.15)$ is obvious and the commutation relation (2.7) is readily verified. Here we have

$$
H=\beta^{-1} \operatorname{Tr} L
$$

cf. (1.2).

Continuing now with the reasoning in Section 2A, we get from (2.10)

$$
\hat{A}_{j k}\left[\frac{1}{2}\left(\lambda_{j}+\lambda_{k}\right)+\frac{i}{2} \cot \tau\left(\lambda_{j}-\lambda_{k}\right)\right]=\hat{e}_{j} \tilde{e}_{k}
$$

Combining this with (2.18) yields

$$
\rho_{n} \mp \sigma_{n} \cot \tau \geqslant 0, \quad n=1, \cdots, l .
$$

Therefore, any pair of complex-conjugate eigenvalues belongs to the closure of the sector $\exp \left(S_{\tau}\right)$ (recall $\left.(2.27)\right)$. More generally, we claim that 


$$
\sigma(L) \subset \exp \left(S_{\tau}^{c l}\right), \quad \tau=\frac{1}{2} \beta \mu g .
$$

Indeed, from (2.12) and (2.16) we deduce

$$
\begin{aligned}
& i \in\left\{1, \cdots, k_{+}\right\} \Rightarrow \hat{A}_{i i}>0, \quad \hat{e}_{i} \tilde{e}_{i} \geqslant 0 \\
& j \in\left\{N-k_{-}+1, \cdots, N\right\} \Rightarrow \hat{A}_{j j}<0, \quad \hat{e}_{j} \tilde{e}_{j} \leqslant 0
\end{aligned}
$$

so that (2.74) implies any real eigenvalue of $L$ is non-negative. Since $0 \notin \sigma(L)$ due to (2.72), our claim (2.76) follows.

We can now proceed in the same way as in Section 2B. We replace (2.28) by

$$
\Omega_{i} \equiv\left\{P \in \Omega \mid \sigma(L(P)) \subset \exp \left(S_{\tau}\right)\right\}
$$

and then define $\Omega_{b}$ and $\Omega_{r}$ by (2.29) and (2.30). (For $\tau=\pi / 2$ one should replace $\Omega$ 's by $\Omega^{b}$ 's throughout.) The analog of (2.31) reads

$$
\lambda_{j}=r \exp (i \tau), \quad \lambda_{k}=\operatorname{rexp}(-i \tau), \quad r>0 \Rightarrow \hat{e}_{j}=\tilde{e}_{k}=0
$$

the implication following from (2.74) and (2.16). Again, the components of $\hat{e}$ (and hence of $\tilde{e})$ do not vanish on $\Omega_{r}\left(\Omega_{r}^{b}\right.$ for $\left.\tau=\pi / 2\right)$ : Here, (2.74) yields after a straightforward calculation

$$
\hat{A}_{j k}=\hat{e}_{j} C(\beta, \mu,-g ; a, a)_{j k} \tilde{e}_{k} \quad\left(\text { on } \Omega_{r}^{(b)}\right)
$$

where

$$
a_{j} \equiv \beta^{-1} \ln \lambda_{j} \in S_{\mu g / 2}
$$

and $C$ is the Cauchy matrix (B1). Therefore, (2.32) and its $\tau=\pi / 2$ version follow in the same way as before.

Next, we introduce $\hat{E}$ by (2.34) and restrict attention to $\Omega_{r}\left(\Omega_{r}^{b}\right.$ for $\left.\tau=\pi / 2\right)$ henceforth. Then the product matrix (2.35) is well defined and (2.36), (2.37) follow again. Defining $v$ and $\tilde{A}$ by (2.38) and (2.39), we obtain (2.40); also, from (2.80) we infer

$$
\tilde{A}_{j k}=C(\beta, \mu,-g ; a, a)_{j k} v_{k}
$$

Then the properties (2.42) of the vector $v$ follow as before.

The upshot is, that $L$ again gives rise to a vector $a$ in the set $A_{l}$ and a vector $v$ in the set $N_{l}$. Once more, the matrix $\mathscr{P}$ is uniquely determined by (2.36) and 
(2.37), so that we obtain a well-defined map

$$
\mathscr{D}: \Omega_{r}^{(b)} \rightarrow D, \quad\left(x^{+}, x^{-}, p^{+}, p^{-}\right) \mapsto(v, a)
$$

where the data set $D$ is defined by (2.46).

In order to run the map backwards we fix a point $(v, a) \in D_{l}$, introduce

$$
\begin{aligned}
& \tilde{A}(v, a)_{j k} \equiv C(\beta, \mu,-g ; a, a)_{j k} v_{k} \\
& \tilde{L}(a) \equiv \operatorname{diag}\left(\exp \left(\beta a_{1}\right), \cdots, \exp \left(\beta a_{N}\right)\right)
\end{aligned}
$$

and verify the commutation relation

$$
c[\tilde{A}, \tilde{L}]=\zeta \otimes v-(\tilde{A} \tilde{L}+\tilde{L} \tilde{A}) / 2 .
$$

Defining $S$ by (2.50)-(2.53) and $A_{s}$ by (2.54), and invoking Lemma B1 once more, we infer $A_{s} \hat{\mathscr{J}}$ is positive. Then both the existence of a regular matrix $\mathscr{M}$ satisfying (2.56) and the properties (2.57) of the eigenvalues of $\tilde{A}$ follow again from Prop.A1.

Transforming (2.86) we now obtain

$$
\left(\mathscr{M}^{-1} \tilde{L} \mathscr{M}\right)_{j k}\left[-i \cos \tau\left(\alpha_{j}-\alpha_{k}\right)+\sin \tau\left(\alpha_{j}+\alpha_{k}\right)\right]=2 \sin \tau\left(\mathscr{M}^{-1} \zeta\right)_{j}\left(\mathscr{M}^{t} v\right)_{k}
$$

Clearly, the square bracket factor is non-zero on $D$ when $\tau \in(0, \pi / 2)$. However, when $\tau=\pi / 2$ the square bracket vanishes for those $(v, a) \in D$ for which $\tilde{A}$ has eigenvalues $\alpha_{j}, \alpha_{k}$ with $\alpha_{j}+\alpha_{k}=0$. Correspondingly, we introduce

$$
D_{(l)}^{b} \equiv\left\{(v, a) \in D_{(l)} \mid \alpha_{i} \neq-\alpha_{j}\right\} \quad(\tau=\pi / 2)
$$

and observe that all square brackets are non-zero on $D^{b}$. Writing $\alpha_{k}$ as

$$
\alpha_{k}=\exp \left(\mu x_{k}\right), \quad \operatorname{Im} x_{k} \equiv\left\{\begin{array}{c}
0 \\
\pi / \mu
\end{array} \quad k=\left\{\begin{array}{c}
1, \cdots, N_{+} \\
N_{+}+1, \cdots, N
\end{array}\right.\right.
$$

we obtain from (2.87) by using (B1)

$$
\left(\mathscr{M}^{-1} \tilde{L} \mathscr{M}\right)_{j k}=\left(\mathscr{M}^{-1} \zeta\right)_{j} C(\mu, \beta, g ; x, x)_{j k}\left(\mathscr{M}^{t} v\right)_{k} .
$$

(Here, we take $(v, a) \in D_{l}^{b}$ for $\tau=\pi / 2$.) Now we have $|\tilde{L}| \neq 0$ in view of (2.85). Hence, the Cauchy matrix is invertible and we have 


$$
\left(\mathscr{M}^{-1} \zeta\right)_{j}\left(\mathscr{M}^{t} v\right)_{j}=\left(\mathscr{M}^{-1} \tilde{L} \mathscr{M}\right)_{j j} \exp \left(\mu x_{j}\right) \neq 0, \quad j=1, \cdots, N
$$

From Cauchy's identity (B4) we may now deduce $x_{j} \neq x_{k}$ for $j \neq k$. But then $\sigma(\tilde{A})$ is simple, and so we may order the eigenvalues $\alpha_{1}, \cdots, \alpha_{N}$ such that the vectors $x^{+}, x^{-}$defined via (2.60), (2.61) satisfy (2.62). (Note that $x_{i}^{+} \neq x_{j}^{-}$for $\tau=\pi / 2$, since we are restricting attention to $D^{b}$ in that case.)

Next, consider the diagonal elements in (2.91). We claim that these are positive. Taking this for granted, we obtain uniquely determined real numbers $p_{i}^{+}$and $p_{j}^{-}$by requiring

$$
\begin{aligned}
& \exp \left(\beta p_{i}^{+}\right) V_{i}^{+}\left(x^{+}, x^{-}\right)=\left(\mathscr{M}^{-1} \tilde{L} \mathscr{M}\right)_{i i}, \quad i=1, \cdots, N_{+} \\
& \exp \left(\beta p_{j}^{-}\right) V_{j}^{-}\left(x^{+}, x^{-}\right)=\left(\mathscr{M}^{-1} \tilde{L} \mathscr{M}\right)_{N_{+}+j, N_{+}+j}, \quad j=1, \cdots, N_{-} .
\end{aligned}
$$

(Since $x_{i}^{+} \neq x_{j}^{-}$for $\tau=\pi / 2$, the potentials $V_{i}^{+}, V_{j}^{-}$are also positive for $\tau=\pi / 2$, cf. (1.3), (1.4).)

To prove the claim, we first note that we may repeat the reasoning below (2.64) to conclude that the diagonal elements are real. Moreover, we may choose the $\mathscr{J}$-unitary $V$ in such a way that (2.91) still holds true when $\mathscr{M}$ is replaced by $S^{-1} K V$ at the lhs. (This amounts to choosing an appropriate permutation matrix commuting with $\mathscr{J}$.) Now from (2.50)-(2.53) one readily verifies

$$
\mathscr{J} K S^{-1} v=K \bar{S} \zeta
$$

Therefore, we may infer

$$
\left(S^{-1} K V\right)^{t} v=\mathscr{J} \bar{V}^{-1} \mathscr{J} K S^{-1} v=\mathscr{J}\left(\overline{S^{-1} K V}\right)^{-1} \zeta
$$

where we used the $\mathscr{J}$-unitarity of $V$. This implies that the lhs of (2.91) is positive (negative) for $j \leqslant N_{+}\left(>N_{+}\right)$, and since this also holds true for the numbers $\exp \left(\mu x_{j}\right)$, the above claim is proved.

The upshot is, that for $\tau \in(0, \pi / 2)$ we obtain a map $\mathscr{J}$ given by (2.65), while for $\tau=\pi / 2$ we get a map

$$
\mathscr{J}: D^{b} \rightarrow \Omega^{b},(v, a) \mapsto\left(x^{+}, x^{-}, p^{+}, p^{-}\right) \quad(\tau=\pi / 2) .
$$

Lemma 2.2. For $\tau \in(0, \pi / 2)$ the assertion of Lemma 2.1 holds true. Now let $\tau=\pi / 2$. Then the map $\mathscr{J}$ is a bijection onto $\Omega_{r}^{b}$ whose inverse is the map $\mathscr{D}$.

Proof. In view of (2.91) we may and will fix the gauge ambiguity in $\mathscr{M}$ by 
requiring (2.66), where $e$ is now defined via (2.69). Combining (2.91)-(2.93) we then obtain again (2.67). The arguments in the proof of Lemma 2.1 now apply with obvious changes that need not be spelled out.

\section{Holomorphy, Canonicity and Monodromy}

\section{A. The Case $\tilde{\mathbf{I}}_{\mathrm{nr}}$}

In this section we are concerned with two vector-valued functions $\mathscr{V}$ and $\eta$ and two matrix-valued functions $\mathscr{L}$ and $\mathscr{A}$ whose arguments $(\hat{q}, \hat{\theta})$ belong to a domain $\mathscr{H} \subset C^{2 N}$ in which they are holomophic. (Here and below, 'domain' stands for 'open and connected set'.) The functions $v, \tilde{A}$ and $\tilde{L}$ on the data set $D$ of Section $2 \mathrm{~B}$ are related to restrictions of the holomorphic functions $\eta, \mathscr{L}$ and $\mathscr{A}$, resp., to subsets of $\mathscr{H}$ with $2 N$ real dimensions.

We first introduce the vector-valued potential function $\mathscr{V}$ by

$$
\mathscr{V}_{k} \equiv(-)^{k-1} \prod_{j \neq k}\left[\left(\hat{\theta}_{k}-\hat{\theta}_{j}\right)^{2}+\mu^{2} g^{2}\right]^{1 / 2} /\left(\hat{\theta}_{k}-\hat{\theta}_{j}\right), \quad k=1, \cdots, N, \quad \mu, g \in(0, \infty)
$$

Clearly, $\mathscr{V}_{k}$ is well-defined, holomorphic and non-zero in the domain

$$
\mathscr{H}_{\mathscr{r}} \equiv\left\{\hat{\theta} \in C^{N} \mid \hat{\theta}_{j} \in S_{\mu g / 2}, \hat{\theta}_{j} \neq \hat{\theta}_{l}, j \neq l, j, l=1, \cdots, N\right\}
$$

(the strip $S_{\mu g / 2}$ being defined by (2.27)), provided we fix the square-root sign ambiguities. (Indeed, all radicands in (3.1) stay away from $(-\infty, 0]$ on $\mathscr{H}_{\boldsymbol{\gamma}}$.) This we do by requiring the square roots to be positive when $\hat{\theta}_{k}-\hat{\theta}_{j}$ is real. For later use we note that this convention ensures

$$
\mathscr{V}_{k}(\hat{\theta})>0, \quad k=1, \cdots, N, \quad \hat{\theta} \in G_{N},
$$

where $G_{N}$ is defined by (1.8).

Next, we introduce the functions

$$
\begin{aligned}
& \eta_{k} \equiv \exp \left(\mu \hat{q}_{k}\right) \mathscr{V}_{k}(\hat{\theta}) \\
& \mathscr{L}_{j k} \equiv C(0, \mu,-g ; \hat{\theta}, \hat{\theta})_{j k} \eta_{k}(\hat{q}, \hat{\theta}) \\
& \mathscr{A} \equiv \operatorname{diag}\left(\hat{\theta}_{1}, \cdots, \hat{\theta}_{N}\right)
\end{aligned}
$$

which are clearly holomorphic in the domain 


$$
\mathscr{H} \equiv\left\{(\hat{q}, \hat{\theta}) \in C^{2 N} \mid \hat{q} \in C^{N}, \hat{\theta} \in \mathscr{H}_{\mathscr{r}}\right\}
$$

It is readily verified that on $\mathscr{H}$ one has

$$
\begin{gathered}
{[\mathscr{L}, \mathscr{A}]=i \mu g(\zeta \otimes \eta-\mathscr{L}), \quad \zeta \equiv(1, \cdots, 1)} \\
|\mathscr{L}|=\exp \mu\left(\hat{q}_{1}+\cdots+\hat{q}_{N}\right),
\end{gathered}
$$

the last equality following from Cauchy's identity (B4).

We continue by introducing the set

$$
\mathscr{H}_{s} \equiv\{P \in \mathscr{H} \mid \mathscr{L}(P) \text { has simple spectrum }\} .
$$

Using well-known results from several complex variables analysis, one readily deduces that $\mathscr{H}_{s}$ is a domain, and that the exceptional set

$$
\mathscr{H}_{e} \equiv \mathscr{H} \backslash \mathscr{H}_{s}
$$

is nowhere dense and has measure zero. Indeed, $\mathscr{H}_{e}$ equals the zero locus of the discriminant of the characteristic polynomial of $\mathscr{L}$. If the discriminant would vanish identically on $\mathscr{H}$, then the discriminant of the polynomial $\mid \operatorname{diag}\left(\exp \left(\mu \hat{q}_{1}\right)\right.$, $\left.\cdots, \exp \left(\mu \hat{q}_{N}\right)\right)-\alpha \mathbf{1}_{N} \mid$ would vanish for any $\hat{q} \in C^{N}$. (This follows e.g. by setting $\hat{\theta} \in G_{N}$ in (3.5) and then taking $\hat{\theta}_{k}-\hat{\theta}_{k+1} \rightarrow \infty$.) Since this is clearly false, $\mathscr{H}_{e}$ is empty or an analytic subvariety of $\mathscr{H}_{s}$ with (complex) codimension one. In either case the assertions follow. (In fact, $\mathscr{H}_{e}$ is not empty, but we do not need to know this.)

Lemma 3.1. Suppose that $\mathscr{U}$ is a subset of $\mathscr{H}_{s}$ such that any two points of $\mathscr{U}$ can be connected by a path in $\mathscr{U}$ consisting of finitely many line segments. If in addition $\mathscr{U}$ is simply-connected or $\mathscr{L}$ has real spectrum on $\mathscr{U}$, then there exists a function $\mathscr{B}: \mathscr{U} \rightarrow G L(N, C)$ that is holomorphic in $\mathscr{U}$ and satisfies

$$
\begin{aligned}
& \left(\mathscr{B}^{-1} \mathscr{L} \mathscr{B}\right)_{j k}=0, \quad j \neq k \\
& \mathscr{B} \zeta=\zeta, \quad \zeta \equiv(1, \cdots, 1) .
\end{aligned}
$$

Poof. Fix $P \in \mathscr{U}$. Since $\mathscr{L}$ has simple spectrum and is holomorphic in $P$, there exists a polydisc $D \subset \mathscr{H}_{s}$ around $P$ such that $\mathscr{L}$ has eigenvalues $\alpha_{1}, \cdots, \alpha_{N}$ and corresponding eigenvectors $u_{1}, \cdots, u_{N}$ that are holomorphic in $D$. Hence, the function 


$$
\mathscr{B} \equiv \operatorname{Col}\left(u_{1}, \cdots, u_{N}\right) \in G L(N, C)
$$

satisfies (3.12); also, $\mathscr{B}$ and the vector $\mathscr{B}^{-1} \zeta$ are holomorphic in $D$. We claim that $\mathscr{B}^{-1} \zeta$ has non-vanishing components. Indeed, when we transform the commutation relation (3.8) with $\mathscr{B}$, we obtain (2.58) and (2.59) with $\mathscr{M}, \tilde{L}$ and $v$ replaced by $\mathscr{B}, \mathscr{A}$ and $\eta$, so that the claim follows. Multiplying $\mathscr{B}$ from the right by the matrix diag $\left(\left(\mathscr{B}^{-1} \zeta\right)_{1}, \cdots,\left(\mathscr{B}^{-1} \zeta\right)_{N}\right)$ we obtain a holomorphic $G L(N, C)$ valued function in $D$ (again denoted $\mathscr{B}$ ) that satisfies (3.12) and (3.13) on $D$.

Obviously, any other holomorphic function $\mathscr{B}^{\prime}: D \rightarrow G L(N, C)$ that satisfies (3.12) and (3.13) must be related to $\mathscr{B}$ via $\mathscr{B}^{\prime}=\mathscr{B} \Pi_{\sigma}$, where $\Pi_{\sigma} \in O(N)$ is a permutation matrix. When $\mathscr{L}$ has real spectrum on $\mathscr{U}$, we may and will render $\mathscr{B}$ unique by ordering the eigenvalues in the point $P \in \mathscr{U}$. Specifically, we shall require $\alpha_{N}<\cdots<\alpha_{1}$.

Now choose a second point $P^{\prime} \in \mathscr{U}$ and a polygonal non-self-intersecting path $\Gamma \subset \mathscr{U}$ connecting $P$ and $P^{\prime}$. For any point on $\Gamma$ we can repeat the above construction, and eventually shrinking the radii of the polydisc we may assume that its intersection with $\Gamma$ is connected. A compactness argument now shows that finitely many polydiscs $D\left(P_{0}\right), D\left(P_{1}\right), \cdots, D\left(P_{n}\right)$ cover $\Gamma$, with $P_{0} \equiv P, P_{n} \equiv P^{\prime}$.

Next, we introduce the connected sets $D_{j} \equiv D\left(P_{j}\right) \cap \Gamma$ and $D_{j k} \equiv D_{j} \cap D_{k}$. Whenever $D_{j k}$ is non-empty, there exists a permutation matrix $\Pi_{j k}$ such that $\mathscr{B}_{j}=\mathscr{B}_{k} \Pi_{j k}$ on $D_{j k}$. Now if $\mathscr{U}$ is simply-connected, we may invoke the monodromy theorem to conclude the proof. Indeed, in that case we can redefine $\mathscr{B}_{1}, \cdots, \mathscr{B}_{n}$ such that $\Pi_{j k}=\mathbb{1}_{N}$ on $D_{j k}$. If $\mathscr{L}$ has real spectrum on $\mathscr{U}$, then our ordering convention ensures $\alpha_{N}<\cdots<\alpha_{1}$ in $P_{j}, j=0, \cdots, N$. Since $\mathscr{B}_{j}$ is continuous and $D_{j}$ connected, it follows that $\alpha_{N}<\cdots<\alpha_{1}$ on $D_{j}$, so that $\Pi_{j k}$ must equal $\mathbb{1}_{N}$

To proceed, we exploit some results from Section $2 \mathrm{~B}$ pertaining to the 'pure soliton' case $N_{-}=0$ (already dealt with in I). Let us denote the data set for this special case by $D_{\mathrm{I}}$, and let us reparametrize $D_{\mathrm{I}}$ with $2 N$ real coordinates $p_{i}^{s}, x_{i}^{s}$ defined by

$$
\left.\begin{array}{l}
a_{i} \equiv p_{i}^{s} \\
v_{i} \equiv \exp \left(\mu x_{i}^{s}\right) \mathscr{V}_{i}\left(p^{s}\right)
\end{array}\right\} \quad i=1, \cdots, N
$$

To verify that the numbers $x_{i}^{s}$ may indeed be chosen real, one need only recall (3.3) and the fact that $a \in G_{N}$ and $v_{i}>0$ for any $(v, a) \in D_{\mathrm{l}}$, cf. Section 2B. The definition (3.15) amounts to a bijection 


$$
\mathscr{C}_{\mathrm{I}}: D_{\mathrm{I}}=(0, \infty)^{N} \times G_{N} \rightarrow \boldsymbol{R}^{N} \times G_{N}, \quad(v, a) \mapsto\left(x^{s}, p^{s}\right)
$$

which is clearly such that

$$
\begin{gathered}
v=\eta\left(x^{s}, p^{s}\right) \\
\tilde{A}(v, a)=\mathscr{L}\left(x^{s}, p^{s}\right) \\
\tilde{L}(a)=\mathscr{A}\left(p^{s}\right) .
\end{gathered}
$$

It now readily follows from (3.18) that $\mathscr{L}$ has positive and simple spectrum on the set

$$
\mathscr{U}_{1} \equiv \boldsymbol{R}^{N} \times G_{N}
$$

so that $\mathscr{U}_{\mathrm{I}} \subset \mathscr{H}_{s}$. Indeed, combining (2.56) and (2.60) we obtain for $N_{-}=0$

$$
\left(\mathscr{M}^{-1} \tilde{A} \mathscr{M}\right)(v, a)=\operatorname{diag}\left(\exp \left(\mu x_{1}^{+}\right), \cdots, \exp \left(\mu x_{N}^{+}\right)\right)
$$

whence the assertion is clear. Therefore, we may invoke Lemma 3.1 to conclude that there exists a unique real-analytic function $\mathscr{B}_{1}: \mathscr{U}_{1} \rightarrow G L(N, C)$ obeying

$$
\left(\mathscr{B}_{1}^{-1} \mathscr{L} \mathscr{B}_{1}\right)\left(x^{s}, p^{s}\right)=\operatorname{diag}\left(\exp \left(\mu x_{1}^{+}\right), \cdots, \exp \left(\mu x_{N}^{+}\right)\right), \quad \mathscr{B}_{1} \zeta=\zeta .
$$

Combining (3.21) and (3.22), it now follows that there exists a diagonal matrix $D$ such that

$$
\mathscr{B}_{1}\left(x^{s}, p^{s}\right)=\mathscr{M}(v, a) D\left(x^{s}, p^{s}\right) .
$$

But then we may infer that

$$
\left(\mathscr{B}_{1}^{-1} \mathscr{A} \mathscr{B}\right)_{i i}\left(x^{s}, p^{s}\right)=\left(\mathscr{M}^{-1} \tilde{L} \mathscr{M}\right)_{i i}(v, a)=p_{i}^{+}, \quad i=1, \cdots, N
$$

where we used (3.19) and (2.63).

Having established the relevant properties of $\mathscr{B}_{1}$ on the set $\mathscr{U}_{\mathfrak{I}}$, we now invoke Lemma 3.1 once more to conclude that $\mathscr{B}_{1}$ can be analytically continued along paths in $\mathscr{H}_{s}$. This gives rise to a (multi-valued) holomorphic function in $\mathscr{H}_{s}$ that will be denoted by $\mathscr{R}$. As a result, the functions

$$
\begin{aligned}
q_{i} & \equiv \mu^{-1} \ln \left(\mathscr{B}_{1}^{-1} \mathscr{L} \mathscr{B}\right)_{i i}, \quad i=1, \cdots, N \\
\theta_{i} & \equiv\left(\mathscr{B}_{1}^{-1} \mathscr{A} \mathscr{B}_{1}\right)_{i i},
\end{aligned}
$$


(where the logarithms are chosen real) likewise extend to holomorphic functions in $\mathscr{H}_{s}$, giving rise to a holomorphic map

$$
R: \mathscr{H}_{s} \rightarrow C^{2 N}, \quad(\hat{q}, \hat{\theta}) \mapsto(q, \theta) .
$$

Before continuing with Lemma 3.2, let us summarize the above notation and constructions in the following commutative diagram:

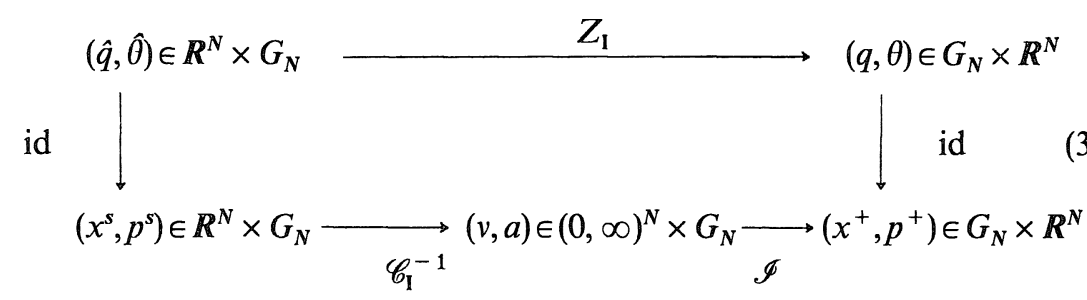

Here, the vertical maps are the identity maps, the map $\mathscr{I}$ is the inverse transform from Section $2 \mathrm{~B}$, the map $\mathscr{C}_{\mathrm{I}}$ is defined via (3.15), (3.16), and the map $Z_{1}$ is the branch (Zweig) of $R$ over $\mathscr{U}_{\mathrm{I}}$ that corresponds to the functions $q$ and $\theta$ as initially defined on $\mathscr{U}_{\mathrm{r}}$. The commutativity of the diagram amounts to the fact that the values of the latter functions coincide with $x^{+}$and $p^{+}$, cf. (3.22), (3.24). (At this stage part of the notation and all of the diagram may seem superfluous. However, we are anticipating notation and diagrams in Chapters 4 and 5 that will be indispensable in keeping track of the more elaborate state of affairs encountered there.)

We are now prepared for the following key lemma, whose proof hinges on results established in $\mathbb{I}$.

Lemma 3.2. The multi-valued holomorphic map $R$ defined by (3.27) admits a local holomorphic inverse and satisfies

$$
\left.\begin{array}{l}
\sum_{j=1}^{N}\left[\frac{\partial y_{k}}{\partial \hat{q}_{j}} \frac{\partial y_{l}}{\partial \hat{\theta}_{j}}-(\hat{q} \leftrightarrow \hat{\theta})\right]=0, \quad y=q, \theta \\
\sum_{j=1}^{N}\left[\frac{\partial q_{k}}{\partial \hat{q}_{j}} \frac{\partial \theta_{l}}{\partial \hat{\theta}_{j}}-(\hat{q} \leftrightarrow \hat{\theta})\right]=\delta_{k l}
\end{array}\right\} \begin{aligned}
& k, l=1, \cdots, N . \\
& k
\end{aligned}
$$

Proof. The map $Z_{\mathrm{I}}$ in the diagram (3.28) coincides with the map denoted by $\mathscr{E}$ in $\mathrm{I}$ (for the $\mathrm{II}_{\mathrm{nr}}$ case). Now we have proved in $\mathrm{I}$ that $\mathscr{E}$ is symplectic, in 
the sense that the equations (3.29) hold true on $\mathscr{U}_{1}$, cf. App. C in I. Clearly, these relations can be analytically continued along paths in $\mathscr{H}_{s}$, so that it remains to show that the matrix

$$
D R \equiv\left[\begin{array}{ll}
\partial_{\hat{q}} q & \partial_{\hat{\theta}} q \\
\partial_{\hat{q}} \theta & \partial_{\hat{\theta}} \theta
\end{array}\right]
$$

is invertible on $\mathscr{H}_{s^{*}}$. But the canonicity relations (3.29) can be rewritten

$$
(D R) \mathscr{S}(D R)^{t}=\mathscr{S}, \quad \mathscr{S} \equiv\left[\begin{array}{cc}
0 & \mathbb{1}_{N} \\
-\mathbb{1}_{N} & 0
\end{array}\right]
$$

so that $|D R| \neq 0$ on $\mathscr{H}_{s}$.

To conclude this section, we shall determine the relation between the values of $\mathscr{R}$ and $R$ lying over any point in $\mathscr{H}_{s}$. From Lemma 3.1 we see that the values of $\mathscr{R}$ must be related by permutation matrices $\Pi_{\sigma}, \sigma \in S_{N}$. The monodromy of $\mathscr{R}$ on the domain $\mathscr{H}_{s}$ can, therefore, be described by the finite group

$$
M(\mathscr{R}) \equiv\left\{\sigma \in S_{N} \mid \text { there exist branches } \mathscr{B}, \mathscr{B}^{\prime} \text { of } \mathscr{R} \text { related by } \mathscr{B}^{\prime}=\mathscr{B} \Pi_{\sigma}\right\}
$$

It then follows from (3.25)-(3.27) that the monodromy of $R$ is given by the discrete group

$$
\begin{aligned}
M(R) \equiv\left\{(k, \sigma) \in \mathbb{Z}^{N} \rtimes S_{N} \mid \text { there exist branches }(q, \theta),\right. \\
\\
\left.\left(q^{\prime}, \theta^{\prime}\right) \text { of } R \text { related by } q^{\prime}=q_{\sigma}+2 \pi i k / \mu, \theta^{\prime}=\theta_{\sigma}\right\}
\end{aligned}
$$

(The notation will be clear from context.)

Lemma 3.3. One has

$$
\begin{aligned}
& M(\mathscr{R})=S_{N} \\
& M(R)=E_{N} \rtimes S_{N}
\end{aligned}
$$

where

$$
E_{N} \equiv\left\{k \in Z^{N} \mid k_{1}+\cdots+k_{N}=0\right\} .
$$

Proof. From (3.9) and (3.25) we obtain

$$
\hat{q}_{1}+\cdots+\hat{q}_{N}=q_{1}+\cdots+q_{N} .
$$


Since this relation is preserved under analytic continuation and the $l h s$ is entire, additive multiples of $2 \pi i / \mu$ arising from going around non-contractible loops must sum to zero. Therefore, we need only exhibit loops $\Gamma_{1}, \cdots, \Gamma_{N-1}, \tilde{\Gamma}_{N}$ in $\mathscr{H}_{s}$ starting at a point in $\mathscr{U}_{I}$ which are such that:

(i) looping $\Gamma_{k}$ results in $q_{k}, q_{k+1} \rightarrow q_{k+1}, q_{k}, k=1, \cdots, N-1$;

(ii) looping $\tilde{\Gamma}_{N}$ results in $q_{N-1}, q_{N} \rightarrow q_{N-1}-2 \pi i / \mu, q_{N}+2 \pi i / \mu$.

Indeed, since $S_{N}$ is generated by transpositions, (i) entails that $M(\mathscr{R})$ equals $S_{N}$ and that $M(R)$ contains $S_{N}$; combining this with (ii), it follows that $M(R)$ is given by (3.35).

We shall prove the existence of such loops by reduction to the case $N=2$. Correspondingly, we first take $N=2$ and define loops $\Gamma$ and $\tilde{\Gamma}$ starting in

$$
\begin{aligned}
& \hat{q}_{\frac{1}{2}}=\mu^{-1} \ln d, \quad d>0 \\
& \hat{\theta}_{\frac{1}{2}}= \pm \frac{1}{2} \mu g \cot \gamma, \quad \gamma \in\left(0, \frac{\pi}{2}\right) .
\end{aligned}
$$

Clearly, this point belongs to $\mathscr{U}_{\mathrm{r}}$. The loop $\Gamma$ is defined by taking

$$
\hat{q}_{2}(t)=\mu^{-1}[\ln d \pm i x(t)]
$$

where

$$
x(t) \equiv \gamma\left(1-e^{i t}\right), \quad t \in[0,2 \pi]
$$

Then the eigenvalues of $\mathscr{L}$ along $\Gamma$ are given by

$$
\alpha_{ \pm}=d\left[\cos x \pm\left(\cos ^{2} x-\cos ^{2} \gamma\right)^{1 / 2}\right] / \cos \gamma
$$

From this one readily deduces that one can find $\gamma_{0} \in\left(0, \frac{1}{2}\right)$ such that

$$
\left.\left|\alpha_{ \pm}-d\right|<2 d \gamma, \quad\left|\alpha_{+}-\alpha_{-}\right|>d \gamma, \quad \gamma \in\left(0, \gamma_{0}\right] \quad \text { (on } \Gamma\right) \text {. }
$$

Taking $\gamma \leqslant \gamma_{0}$ from now on, it follows from the first estimate that the eigenvalues stay in the right half plane and from the second one that $\sigma(\mathscr{L})$ remains simple along $\Gamma$. Since $\cos ^{2} x(t)-\cos ^{2} \gamma$ winds once around the origin, it follows that $q_{1}, q_{2} \rightarrow q_{2}, q_{1}$ along $\Gamma$, as advertised.

Next, we define a loop $\tilde{\Gamma}$ by taking

$$
\hat{\theta}_{\frac{1}{2}}(t)= \pm \frac{1}{2} \mu g / \operatorname{sh}(\ln r-i t), \quad r \geqslant 2, \quad t \in[0,2 \pi]
$$


where $r$ is chosen large enough so that $\tilde{\Gamma} \subset \mathscr{H}$. Then the eigenvalues of $\mathscr{L}$ are given by

$$
\alpha_{+}=d r e^{-i t}, \quad \alpha_{-}=\frac{d}{r} e^{i t}, \quad r \geqslant 2
$$

entailing

$$
\left.\left|\alpha_{ \pm}\right| \leqslant d r, \quad\left|\alpha_{+}-\alpha_{-}\right|>d \quad \text { (on } \tilde{\Gamma}\right)
$$

Again, the loop $\tilde{\Gamma}$ stays in $\mathscr{H}_{s}$, but now $\alpha_{+}, \alpha_{-}$return to their values, winding once around the origin; more specifically, we obtain $q_{1}, q_{2} \rightarrow q_{1}-2 \pi i / \mu, q_{2}+2 \pi i / \mu$, as promised.

We are now prepared to handle the general $N$ case. By definition, the loop $\Gamma_{k}$ is given by

$$
\begin{aligned}
& \hat{q}_{j}= \begin{cases}\mu^{-1} \ln (N-j+1), & j \neq k, k+1 \\
\mu^{-1}\left[\ln \left(N-k+\frac{1}{2}\right) \pm i x(t)\right], & j=\left\{\begin{array}{l}
k \\
k+1
\end{array}\right.\end{cases} \\
& \hat{\theta}_{\substack{k+1 \\
k+1}}= \pm \frac{1}{2} \mu g \cot \gamma_{k}, \quad \gamma_{k}=\gamma_{0} /\left(N-k+\frac{1}{2}\right) \\
& \hat{\theta}_{j}-\hat{\theta}_{j+1}=\mu g \Lambda, \quad j \neq k
\end{aligned}
$$

where $\Lambda>0$ is at our disposal. We claim that when $\Lambda$ is chosen large enough, the loop remains in $\mathscr{H}_{s}$ and has the desired property (i).

To prove this claim, we denote $\mathscr{L}$ evaluated in points of $\Gamma_{k}$ by $\mathscr{L}(\Lambda)$ and observe that

$$
\lim _{\Lambda \rightarrow \infty} \mathscr{L}(\Lambda)=\operatorname{diag}\left(N, \cdots, N-k+2, \mathscr{L}_{2}(\Gamma), N-k-1, \cdots, 1\right) \equiv \mathscr{L}(\infty)
$$

Here, $\mathscr{L}_{2}(\Gamma)$ denotes the $N=2$ matrix $\mathscr{L}$ evaluated along the loop $\Gamma$, with $d=N-k+\frac{1}{2}$ and $\gamma=\gamma_{k}$. From the estimates (3.43) we then see that the eigenvalues of $\mathscr{L}(\infty)$ stay a distance at least $\gamma_{0}$ apart along $\Gamma . \quad\left(\right.$ Recall $\left.\gamma_{0}<1 / 2.\right) \quad$ Let us now use the matrix

$$
\mathscr{R}(\infty) \equiv \operatorname{diag}\left(1, \cdots, 1, \mathscr{R}_{2}(\Gamma), 1, \cdots, 1\right)
$$


diagonalizing $\mathscr{L}(\infty)$ along $\Gamma$ to write

$$
\begin{aligned}
\mathscr{R}(\infty)^{-1} \mathscr{L}(\Lambda) \mathscr{R}(\infty)= & \operatorname{diag}\left(N, \cdots, N-k+2, \alpha_{+}, \alpha_{-}, N-k-1, \cdots, 1\right) \\
& +\mathscr{R}(\infty)^{-1}[\mathscr{L}(\Lambda)-\mathscr{L}(\infty)] \mathscr{R}(\infty) \equiv D+V(\Lambda) .
\end{aligned}
$$

The point of this is, that one is then dealing with a perturbation $V(\Lambda)$ of a normal matrix $D$ whose eigenvalues stay at least a distance $\gamma_{0}$ apart along $\Gamma$. Therefore, the above claim will follow if for $\Lambda$ large enough one has $\|V(\Lambda)\|<\gamma_{0} / 2$ along $\Gamma_{k}$. (Here and from now on, $\|\cdot\|$ denotes the norm derived from the standard inner product on $\mathbb{C}^{N}$.) But this is clearly true: One has $\|\mathscr{L}(\Lambda)-\mathscr{L}(\infty)\|=O\left(\Lambda^{-1}\right)$ uniformly on $\mathbb{\Gamma}_{k}$, and $\left\|\mathscr{R}(\infty)^{-1}\right\|,\|\mathscr{R}(\infty)\|$ are independent of $\Lambda$ and bounded on $\Gamma$.

Next, we define $\tilde{\Gamma}_{N}$ by setting

$$
\begin{aligned}
& \hat{q}_{j}= \begin{cases}\mu^{-1} \ln (N-j+1), & j<N-1 \\
\mu^{-1} \ln (1 / \mathrm{r}), & j=N-1, N\end{cases} \\
& \hat{\theta}_{j}= \begin{cases}\mu g(N-j+1) \Lambda, & j<N-1 \\
\pm \frac{1}{2} \mu g / \operatorname{sh}(\ln r-i t), & j=\left\{\begin{array}{l}
N-1 \\
N
\end{array}\right.\end{cases}
\end{aligned}
$$

As before, we assert that choosing $\Lambda$ large enough ensures $\tilde{\Gamma}_{N}$ remains in $\mathscr{H}_{s}$ and has property (ii).

To prove this, we proceed in the same way as for $\Gamma_{k}$, obtaining

$$
\lim _{\Lambda \rightarrow \infty} \mathscr{L}(\Lambda)=\operatorname{diag}\left(N, N-1, \cdots, 3, \mathscr{L}_{2}(\tilde{\Gamma})\right) \equiv \mathscr{L}(\infty)
$$

as the analog of (3.50). Since the number $d$ in $\widetilde{\Gamma}$ equals $1 / r$, it follows from the estimates (3.46) that the eigenvalues of $\mathscr{L}(\infty)$ stay a distance at least $1 / r$ apart on $\tilde{\Gamma}$. Let us now set $k=N-1$ and

$$
\mathscr{R}(\infty) \equiv \operatorname{diag}\left(1, \cdots, 1, \mathscr{R}_{2}(\tilde{\Gamma})\right)
$$

in (3.52), and let us choose $\Lambda$ large enough so that $\|V(\Lambda)\|<1 / r^{2}$ along $\tilde{\Gamma}_{N}$. Since $r \geqslant 2$, it follows as before that $\tilde{\Gamma}_{N} \subset \mathscr{H}_{s^{*}}$ Moreover, the perturbed eigenvalues $\alpha_{ \pm}(\Lambda)$ must still wind around the origin and return to their values, since $\left|\alpha_{+}(\infty)\right|$ and $\left|\alpha_{-}(\infty)\right|$ equal 1 and $1 / r^{2}$, resp. (cf. (3.45)). 


\section{B. The Case $\tilde{\mathbf{I}}_{\text {rel }}$}

We proceed by handling the relativistic case. Here, the potential function $\mathscr{V}$ is defined by

$$
\begin{array}{r}
\mathscr{V}_{k} \equiv(-)^{k-1} \prod_{j \neq k}\left[\operatorname{sh} \frac{1}{2} \beta\left(\hat{\theta}_{k}-\hat{\theta}_{j}+i \mu g\right) \operatorname{sh} \frac{1}{2} \beta\left(\hat{\theta}_{k}-\hat{\theta}_{j}-i \mu g\right)\right]^{1 / 2} / \mathrm{sh} \frac{1}{2} \beta\left(\hat{\theta}_{k}-\hat{\theta}_{j}\right), \\
k=1, \cdots, N
\end{array}
$$

where

$$
(\beta, \mu, g) \in(0, \infty)^{3} \cap\{\tau \in(0, \pi / 2]\}, \quad \tau \equiv \frac{1}{2} \beta \mu g .
$$

Then $\mathscr{V}_{k}$ is again well defined, holomorphic and non-zero on the domain $\mathscr{H}_{v}$ of Section 3A, cf. (3.2); the restriction on $\tau$ ensures that the radicands stay away from $(-\infty, 0]$ on $\mathscr{H}_{\mathscr{r}}$, so that we may again fix the sign ambiguity by requiring the square roots to be positive for real $\hat{\theta}$. Then (3.3) is again clear.

Next, we define

$$
\begin{aligned}
& \eta_{k} \equiv \exp \left(\mu \hat{q}_{k}+\beta \hat{\theta}_{k}\right) \mathscr{V}_{k}(\hat{\theta}) \\
& \mathscr{L}_{j k} \equiv C\left(\beta, \mu,-g ; \hat{\theta}, \hat{\theta}_{j k} \eta_{k}(\hat{q}, \hat{\theta})\right. \\
& \mathscr{A} \equiv \operatorname{diag}\left(\exp \left(\beta \hat{\theta}_{1}\right), \cdots, \exp \left(\beta \hat{\theta}_{N}\right)\right) .
\end{aligned}
$$

Then $\eta, \mathscr{L}$ and $\mathscr{A}$ are holomorphic in $\mathscr{H}$, cf. (3.7), and on $\mathscr{H}$ one has the relations

$$
-i \cot \tau[\mathscr{L}, \mathscr{A}]=2 \zeta \otimes \eta-\mathscr{L} \mathscr{A}-\mathscr{A} \mathscr{L}
$$

and (3.9), cf. (B4). Introducing $\mathscr{H}_{s}$ and $\mathscr{H}_{e}$ by (3.10) and (3.11), it follows as before that $\mathscr{H}_{e}$ is empty or a codimension-one analytic subvariety, so that $\mathscr{H}_{s}$ is a domain. Now let $\alpha_{1}, \cdots, \alpha_{N}$ be the eigenvalues of $\mathscr{L}$ in some point $P \in \mathscr{H}_{s}$. Then $\alpha_{k}$ extends to a multi-valued holomorphic function in $\mathscr{H}_{s}$, the values lying over any point yielding some subset of $\left\{\alpha_{1}, \cdots, \alpha_{N}\right\}$. (As a matter of fact, this subset equals $\left\{\alpha_{1}, \cdots, \alpha_{N}\right\}$ for any point, cf. Lemma 3.6 below. However, this fact is not yet needed.) But the function

$$
\mathscr{F} \equiv \prod_{1 \leqslant j<k \leqslant N}\left[\left(\alpha_{j}-\alpha_{k}\right)^{2}+4 \alpha_{j} \alpha_{k} \sin ^{2} \tau\right]
$$

is invariant under permutations and hence one-valued on $\mathscr{H}_{s}$. Since $\mathscr{F}$ is not identically zero, its zero locus 


$$
\mathscr{N}_{\mathscr{F}} \equiv\left\{P \in \mathscr{H}_{\boldsymbol{s}} \mid \mathscr{F}(P)=0\right\}
$$

is empty or a codimension-one analytic subvariety of $\mathscr{H}_{s}$. In either case, the set

$$
\mathscr{H}_{s}^{b} \equiv \mathscr{H}_{s} \backslash \mathcal{N}_{\mathscr{F}}
$$

is a domain.

Lemma 3.4. With $\mathscr{H}_{s}$ replaced by $\mathscr{H}_{s}^{b}$, the assertions of Lemma 3.1 hold true.

Proof. We follow the reasoning in the proof of Lemma 3.1. Here, the commutation relation (3.62), transformed with $\mathscr{B}$, yields

$$
\left(\mathscr{B}^{-1} \mathscr{A} \mathscr{B}\right)_{j k}\left[e^{i \tau} \alpha_{j}-e^{-i \tau} \alpha_{k}\right]=2 i \sin \tau\left(\mathscr{B}^{-1} \zeta\right)_{j}\left(\mathscr{B}^{t} \eta\right)_{k}
$$

Since $\mathscr{F} \neq 0$ on $\mathscr{H}_{s}^{b}$, we may now invoke (B3) to deduce

$$
\left(\mathscr{B}^{-1} \zeta\right)_{j}\left(\mathscr{B}^{t} \eta\right)_{j}=\left(\mathscr{B}^{-1} \mathscr{A} \mathscr{B}\right)_{j j} \alpha_{j} \neq 0, \quad j=1, \cdots, N
$$

(Cf. the arguments leading to (2.91).) Then the proof can be completed as before.

We can now proceed as in the previous case. Thus, we reparametrize the $N_{-}=0$ data set $D_{\mathrm{I}}$ by setting

$$
\left.\begin{array}{l}
a_{i} \equiv p_{i}^{s} \\
v_{i} \equiv \exp \left(\mu x_{i}^{s}+\beta p_{i}^{s}\right) \mathscr{V}_{i}\left(p^{s}\right)
\end{array}\right\} \quad i=1, \cdots, N
$$

and then (3.17)-(3.19) again hold true. Also, since (3.21) is still valid, it follows as before that $\mathscr{L}$ has positive and simple spectrum on the set $\mathscr{U}_{\mathrm{r}}$. Therefore, a unique real-analytic function $\mathscr{B}_{1}: \mathscr{U}_{1} \rightarrow G L(N, C)$ satisfying (3.22) exists again, and $\mathscr{B}_{1}$ is related to the matrix $\mathscr{M}$ of Section $2 \mathrm{C}$ via a diagonal matrix $D$ as specified in (3.23). As a consequence we obtain

$$
\left(\mathscr{B}_{1}^{-1} \mathscr{A} \mathscr{B}_{1}\right)_{i i}\left(x^{s}, p^{s}\right)=\left(\mathscr{M}^{-1} \tilde{L} \mathscr{M}\right)_{i i}(v, a)=\exp \left(\beta p_{i}^{+}\right) V_{i}^{+}\left(x^{+}\right), \quad i=1, \cdots, N
$$

cf. (2.92).

Now $\mathscr{B}_{1}$ gives rise to a holomorphic function $\mathscr{R}$ in $\mathscr{H}_{s}^{b}$ in the same way as before. We proceed by introducing the functions

$$
q_{i} \equiv \mu^{-1} \ln \alpha_{i}, \quad \alpha_{i} \equiv\left(\mathscr{B}_{1}^{-1} \mathscr{L} \mathscr{B}_{1}\right)_{i i}
$$




$$
\theta_{i} \equiv \beta^{-1} \ln \left(\mathscr{B}_{1}^{-1} \mathscr{A} \mathscr{B}_{1}\right)_{i i}-\frac{1}{2} \beta^{-1} \ln \left(\prod_{k \neq i}\left[\frac{\left(e^{i \tau} \alpha_{i}-e^{-i \tau} \alpha_{k}\right)\left(e^{-i \tau} \alpha_{i}-e^{i \tau} \alpha_{k}\right)}{\left(\alpha_{i}-\alpha_{k}\right)^{2}}\right]\right)
$$

Here, all logarithms can and will be chosen real. Then we have again

$$
q_{i}=x_{i}^{+}, \quad \theta_{i}=p_{i}^{+}, \quad i=1, \cdots, N \quad\left(\text { on } \mathscr{U}_{\mathrm{I}}\right)
$$

cf. (3.22), (3.69), (1.3). Since the arguments of all logarithms are non-zero on $\mathscr{H}_{s}^{b}$ (recall (3.67) and (3.63)-(3.65)), analytic continuation to $\mathscr{H}_{s}^{b}$ does not meet obstructions, and hence a multi-valued holomorphic map

$$
R: \mathscr{H}_{s}^{b} \rightarrow C^{2 N}, \quad(\hat{q}, \hat{\theta}) \mapsto(q, \theta)
$$

arises. Then the diagram (3.28) again applies, commutativity being a consequence of (3.72).

Before continuing with Lemma 3.5, we would like to point out that (3.71) can be rewritten

$$
\left(\mathscr{B}_{1}^{-1} \mathscr{A} \mathscr{B}_{1}\right)_{k k}=\exp \left(\beta \theta_{k}\right) \mathscr{V}_{k}(\mu, \beta, g ; q) \quad\left(\text { on } \mathscr{U}_{\mathrm{I}}\right)
$$

where $\mathscr{V}_{k}(\beta, \mu, g ; \hat{\theta})$ is defined by (3.57). This fact is only one aspect of the self-duality of the $\mathrm{II}_{\mathrm{rel}}$ case, cf. $\mathrm{I}$. However, in this paper we have opted for denoting the $(q, \theta)$-space and $(\hat{q}, \hat{\theta})$-space objects by different symbols. Recall in this connection that we have restricted the definition domain of the function $\mathscr{V}_{k}(\beta, \mu, g ; \hat{\theta})$ so that it remains one-valued. Therefore, the function $\mathscr{V}_{k}(\mu, \beta, g ; q)$ would no longer be well defined when the imaginary part of $q_{j}$ gets large (compared to $|\beta g|$ ), and such $q$-values do arise in the $\widetilde{I}_{\text {rel }}$ case, cf. also (1.3), (1.4).

Lemma 3.5. The assertions of Lemma 3.2 hold true, with $R$ being the map (3.73).

Proof. The proof of Lemma 3.2 applies with obvious changes.

It is again clear that the monodromy of $\mathscr{R}$ on $\mathscr{H}_{s}^{b}$ can be encoded in the finite group $M(\mathscr{R})$ defined by (3.32). (We shall presently prove $M(\mathscr{R})=S_{N}$.) However, in the case at hand not only the definition of $q$ but also that of $\theta$ involves logarithms; specifically, we conclude from (3.70) and (3.71) that (3.33) should be replaced by 


$$
\begin{gathered}
M(R) \equiv\left\{(k, n, \sigma) \in\left(Z^{N} \times Z^{N}\right) \rtimes S_{N} \mid \text { there exist branches of } R\right. \text { related by } \\
\left.\qquad q^{\prime}=q_{\sigma}+2 \pi i k / \mu, \theta^{\prime}=\theta_{\sigma}+\pi i n / \beta\right\} .
\end{gathered}
$$

Just as in the previous case, the additive multiples must add up to 0 by virtue of the relations

$$
\Sigma \hat{q}_{j}=\Sigma q_{j}, \quad \Sigma \hat{\theta}_{j}=\Sigma \theta_{j}
$$

(The first equality follows again by writing $|\mathscr{L}|$ in terms of $\hat{q}$ and $q$, and the second one by writing $|\mathscr{A}|$ in terms of $\hat{\theta}$ and $\theta$, cf. (3.66), (3.74).)

We conjecture that for $\tau \in(0, \pi / 2)$ these sum rules are the only constraints. That is, using obvious notation (cf. (3.36)), we expect

$$
M(R)=\left(E_{N}(q) \times E_{N}(\theta)\right) \rtimes S_{N}, \quad \tau \in(0, \pi / 2)
$$

However, for $\tau=\pi / 2$ it is plausible that the group $M(R)$ is smaller. Specifically, we expect that in this case $M(R)$ is generated by the transformations

$$
\begin{aligned}
& q_{k}, q_{k+1}, \theta_{k}, \theta_{k+1} \mapsto q_{k+1}, q_{k}, \theta_{k+1}+i \pi / \beta, \theta_{k}-i \pi / \beta, \quad k=1, \cdots, N-1 \\
& q_{1}, q_{2} \mapsto q_{1}-2 \pi i / \mu, q_{2}+2 \pi i / \mu .
\end{aligned}
$$

These conjectures hold true for $N=2$, but we skip the proof. Here, we restrict ourselves to handling the $q$-monodromy.

Lemma 3.6. For any $\sigma \in S_{N}$ and $k \in Z^{N}$ with $\Sigma k_{j}=0$ there exist branches $\mathscr{B}$, $\mathscr{B}^{\prime}$ of $\mathscr{R}$ and branches $Z, Z^{\prime}$ of $R$ related by $\mathscr{B}^{\prime}=\mathscr{B} \Pi_{\sigma}$ and $q^{\prime}=q_{\sigma}+2 \pi i k / \mu$, $\theta^{\prime}=\theta_{\sigma}+\pi i n / \beta$, resp. Here, one has $n \in Z$ and $\Sigma n_{j}=0$.

Proof. We follow the reasoning in the proof of Lemma 3.3. The $N=2$ loop $\Gamma$ is defined in the same way as before, except that (3.39) is replaced by

$$
\hat{\theta}_{\frac{1}{2}}= \pm \beta^{-1} \operatorname{arsh}(\sin \tau \cot \gamma), \quad \gamma \in\left(0, \gamma_{0} \tau / 8\right]
$$

Then the eigenvalues of $\mathscr{L}$ are again given by (3.42) and using the bounds (3.43) one readily verifies

$$
\left.\left|e^{i \tau} \alpha_{ \pm}-e^{-i \tau} \alpha_{\mp}\right|>d \sin \tau, \quad \tau \in(0, \pi / 2] \quad \text { (on } \Gamma\right) .
$$

As a consequence, one may infer $\Gamma \subset \mathscr{H}_{s}^{b}$ and $q_{1}, q_{2} \rightarrow q_{2}, q_{1}$ along $\Gamma$. 
The loop $\tilde{\Gamma}$ is defined by replacing (3.44) by

$$
\hat{\theta}_{\frac{1}{2}}(t)= \pm \beta^{-1} \operatorname{arsh}(\sin \tau / \operatorname{sh}(\ln r-i t)), \quad r \geqslant 2, \quad t \in[0,2 \pi]
$$

(Once more, $r$ is chosen large enough to ensure $\hat{\theta}_{j}(t) \in S_{\mu g / 2}$.) Then (3.45) follows again, implying $\tilde{\Gamma} \subset \mathscr{H}_{s}^{b}$ and $q_{1}, q_{2} \rightarrow q_{1}-2 \pi i / \mu, q_{2}+2 \pi i / \mu$.

From the above the validity of the lemma for $N=2$ follows. Continuing with the general case, we replace $(3.48)$ by

$$
\hat{\theta}_{\substack{k+1 \\ k+1}}= \pm \beta^{-1} \operatorname{arsh}\left(\sin \tau \cot \gamma_{k}\right), \quad \gamma_{k} \equiv \gamma_{0} \tau / 8\left(N-k+\frac{1}{2}\right)
$$

and proceed as before. Choosing $\Lambda$ large enough so that $\|V(\Lambda)\|<\gamma_{0} \tau / 16$ along $\Gamma_{k}$, one readily deduces $\Gamma_{k} \subset \mathscr{H}_{s}^{b}$ and the property (i) by using the estimates (3.43) and (3.81).

Finally, in order to define $\tilde{\Gamma}_{N}$ we replace $\hat{\theta}_{N-1}$ in (3.54) by the $r h s$ of (3.82). Choosing $\Lambda$ such that one has $\|V(\Lambda)\|<1 / r^{2}$ along $\tilde{\Gamma}_{N}$ then guarantees $\tilde{\Gamma}_{N} \subset \mathscr{H}_{s}^{b}$ and property (ii). (Recall (3.45) and $d=1 / r$ on $\tilde{\Gamma}_{N}$, cf. (3.53).)

\section{The Action-angle Transform}

\section{A. The Case $\tilde{I}_{n r}$}

Using the results of Section $3 \mathrm{~A}$ as a guide, we continue by reparametrizing the data set $D$ with $2 N$ real coordinates that will be proved to be action-angle variables. Specifically, fixing $(v, a) \in D_{l}$ we set

$$
\begin{aligned}
& \left.\begin{array}{l}
v_{i}=\exp \left(\mu x_{i}^{s}\right)\left|\mathscr{V}_{i}(a)\right| \\
a_{i}=p_{i}^{s}
\end{array}\right\} \quad i=1, \cdots, k_{+} \quad \text { (solitons) } \\
& \left.\begin{array}{l}
v_{N-k_{-}+j}=-\exp \left(\mu x_{j}^{\bar{j}}\right)\left|\mathscr{V}_{N-k_{-}+j}(a)\right| \\
a_{N-k_{-}+j}=p_{j}^{\bar{s}}
\end{array}\right\} \quad j=1, \cdots, k_{-} \quad \text { (antisolitons) (4.2) } \\
& \left.\begin{array}{l}
v_{k_{+}+n}=(-)^{k_{+}+n-1} \exp \left(\mu\left[x_{n}+i \gamma_{n}\right]\right) \mathscr{V}_{k_{+}+n}(a) \\
a_{k_{+}+n}=\frac{1}{2}\left(p_{n}-i \delta_{n}\right)
\end{array}\right\} \quad n=1, \cdots, l \quad \text { (breathers) }
\end{aligned}
$$

(When $N_{-}=0$ we have $k_{-}=l=0$, and (3.15) results. Note also $\mathscr{V}_{i}(a), \mathscr{V}_{N-k_{-}+j}(a)$ 
are real, but not necessarily positive.) In this way we obtain a bijection

$$
\mathscr{C}_{l}: D_{l} \rightarrow \hat{\Omega}_{l},(v, a) \mapsto\left(x^{s}, x^{\bar{s}}, x, \gamma, p^{s}, p^{\bar{s}}, p, \delta\right)
$$

where $\hat{\Omega}_{l}$ can be written

$$
\begin{aligned}
& \hat{\Omega}_{l}=S_{k_{+}, k_{-}} \times B_{l} \\
& S_{k_{+}, k_{-}} \equiv\left\{\left(x^{s}, x^{\bar{s}}, p^{s}, p^{s}\right) \in \boldsymbol{R}^{2\left(k_{+}+k_{-}\right)} \mid p^{s} \in G_{k_{+}}, p^{\bar{s}} \in G_{k_{-}}, p_{i}^{s} \neq p_{j}^{s}\right\} \\
& B_{l} \equiv\left\{(x, \gamma, p, \delta) \in \boldsymbol{R}^{4 l} \mid \gamma_{n} \in(-\pi / \mu, \pi / \mu], \delta_{n} \in(-\mu g, 0),\right. \\
& \left.\quad p_{n}-i \delta_{n} \neq p_{k}-i \delta_{k}, p_{1}-i \delta_{1}, \cdots, p_{l}-i \delta_{l} \text { in lexicographic order }\right\} .
\end{aligned}
$$

Summing over $l$ now yields a set

$$
\hat{\Omega} \equiv \bigcup_{l=0}^{m} \hat{\Omega}_{l}, \quad m \equiv \min \left(N_{+}, N_{-}\right)
$$

and a bijection $\mathscr{C}: D \rightarrow \hat{\Omega}$.

Clearly, the soliton set $S_{k_{+} k_{-}}$is a manifold having $\left(k_{+}+k_{-}\right) ! / k_{+} ! k_{-}$! connected components, the binomial coefficient being the number of distinct interlacings of the soliton and antisoliton momenta. On the other hand, the breather set $B_{l}$ is not a manifold, as it stands. But we may and will reinterpret this set as a coordinatization of a manifold (denoted by the same symbol), as follows.

When we omit the ordering restriction and take $\gamma_{n} \in \boldsymbol{R}$ at the $r h s$ of (4.7), we do obtain a manifold $\tilde{B}_{l}$. On this manifold we may define an action of the symmetric group $S_{l}$ by simultaneous permutation of $x, \gamma, p, \delta \in \mathbb{R}^{l}$, and a $Z^{l}$-action given by

$$
\gamma \mapsto \gamma+2 \pi k / \mu, \quad k \in Z^{l}
$$

In this way we obtain a free action of a semi-direct product of $Z^{l}$ and $S_{l}$. We may and will view $B_{l}$ as the manifold obtained from $\tilde{B}_{l}$ by quotienting out this action:

$$
B_{l} \equiv \tilde{B}_{l} /\left(Z^{l} \rtimes S_{l}\right)
$$

It is to be noted that the covering manifold $\widetilde{B}_{l}$ is not simply-connected for $l>1$.

Next, we turn $\hat{\Omega}_{l}$ into a symplectic manifold by defining the symplectic form 


$$
\hat{\omega}_{l} \equiv \sum_{i=1}^{k_{+}} d x_{i}^{s} \wedge d p_{i}^{s}+\sum_{j=1}^{k_{-}} d x_{j}^{\bar{s}} \wedge d p_{j}^{\bar{s}}+\sum_{n=1}^{l}\left(d x_{n} \wedge d p_{n}+d \gamma_{n} \wedge d \delta_{n}\right)
$$

More precisely, the $r h s$ yields a symplectic form $\tilde{\hat{\omega}}_{l}$ on $S_{k_{+} . k_{-}} \times \tilde{B}_{l}$, w.r.t. which the $\left(Z^{l} \rtimes S_{l}\right)$-action is symplectic. Therefore, $\hat{\omega}_{l}$ descends to a well-defined symplectic form $\hat{\omega}_{l}$ under the covering projection $\widetilde{B}_{l} \rightarrow B_{l}$, for which (4.11) is a convenient shorthand.

We proceed by introducing the subset

$$
\Omega_{l} \equiv \mathscr{I}\left(D_{l}\right)
$$

of the phase space $\Omega$. On $\Omega_{l}$ the matrix $L$ has simple spectrum and $l$ complexconjugate pairs of eigenvalues in the strip $S_{\mu g / 2}$, cf. Section 2 B. Since $L$ is continuous on $\Omega$, the subset $\Omega_{l}$ is open and hence a manifold. Thus, restriction of the symplectic form $\omega$ (defined by (1.7)) to $\Omega_{l}$ yields a symplectic manifold $\left\langle\Omega_{l}, \omega\right\rangle$.

As a preparation for Theorem 4.1 below, we introduce some more notation. We denote the restrictions of the direct and inverse transforms to $\Omega_{l}$ and $D_{l}$ by $\mathscr{D}_{l}$ and $\mathscr{I}_{l}$, resp., and define bijections

$$
\begin{aligned}
& \Phi_{l} \equiv \mathscr{C}_{l} \circ \mathscr{D}_{l}: \Omega_{l} \rightarrow \hat{\Omega}_{l} \\
& \mathscr{E}_{l} \equiv \mathscr{I}_{l} \circ \mathscr{C}_{l}^{-1}: \hat{\Omega}_{l} \rightarrow \Omega_{l} .
\end{aligned}
$$

(Recall $\mathscr{C}_{l}$ is defined by (4.1)-(4.4).) Summing over $l$ now yields the symplectic manifolds

$$
\begin{aligned}
& \left\langle\Omega_{r}, \omega\right\rangle=\bigcup_{l=0}^{m}\left\langle\Omega_{l}, \omega\right\rangle \\
& \langle\hat{\Omega}, \hat{\omega}\rangle \equiv \bigcup_{l=0}^{m}\left\langle\hat{\Omega}_{l}, \hat{\omega}_{l}\right\rangle
\end{aligned}
$$

which are intertwined by the bijections

$$
\begin{aligned}
& \Phi \equiv \mathscr{C} \circ \mathscr{D}: \Omega_{r} \rightarrow \hat{\Omega} \\
& \mathscr{E} \equiv \mathscr{I} \circ \mathscr{C}^{-1}: \hat{\Omega} \rightarrow \Omega_{r} .
\end{aligned}
$$

Theorem 4.1. The maps $\Phi_{l}$ and $\Phi$ are symplectic diffeomorphisms with inverses $\mathscr{E}_{l}$ and $\mathscr{E}$, resp. 
Proof. We need only prove that $\mathscr{E}_{l}$ is a symplectic diffeomorphism from $\left\langle\hat{\Omega}, \hat{\omega}_{l}\right\rangle$ onto $\left\langle\Omega_{l}, \omega\right\rangle, l=0, \cdots, m$. To this end we first construct and then exploit the following commutative diagram:

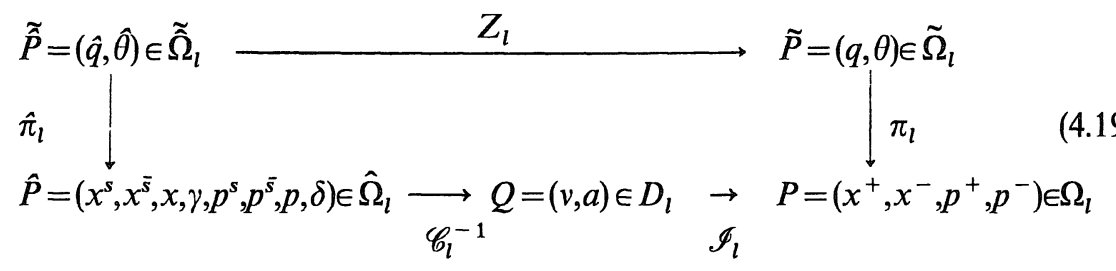

(The definition of this diagram will be such that it reduces to the diagram (3.28) for the special case $N_{-}=0$.) The bottom line of the diagram has already been detailed, so we need only specify $\tilde{\hat{\Omega}}_{l}, \hat{\pi}_{l}, Z_{l}, \tilde{\Omega}_{l}$ and $\pi_{l}$.

First, the set $\tilde{\Omega}_{l}$ consists of all points in $C^{2 N}$ of the following form (from now on, $\epsilon(\cdot)$ denotes the sign function):

$$
\begin{aligned}
& \left(\hat{\theta}_{1}, \cdots, \hat{\theta}_{k_{+}}\right) \in G_{k_{+}},\left(\hat{\theta}_{N-k_{-}+1}, \cdots, \hat{\theta}_{N}\right) \in G_{k_{-}}, \quad \hat{\theta}_{i} \neq \hat{\theta}_{N-k_{-}+j} \\
& \left(\hat{q}_{1}, \cdots, \hat{q}_{k_{+}}\right) \in C^{k_{+}}, \quad \operatorname{Im} \hat{q}_{i} \equiv\left\{\begin{array}{c}
0 \\
\pi / \mu
\end{array} \Leftrightarrow \prod_{j=1}^{k_{-}} \epsilon\left(\hat{\theta}_{i}-\hat{\theta}_{N-k_{-}+j}\right)= \pm 1, i=1, \cdots, k_{+}\right.
\end{aligned}
$$

$\left(\hat{q}_{N-k_{-}+1}, \cdots, \hat{q}_{N}\right) \in C^{k_{-}}, \operatorname{Im} \hat{q}_{N-k_{-}+j} \equiv\left\{\begin{array}{c}\pi / \mu \\ 0\end{array} \Leftrightarrow \prod_{i=1}^{k_{+}} \epsilon\left(\hat{\theta}_{i}-\hat{\theta}_{N-k_{-}+j}\right)= \pm 1, j=1, \cdots, k_{-}\right.$

$$
\begin{aligned}
& \left(\hat{q}_{k_{+}+1}, \cdots, \hat{q}_{k_{+}+l}, \hat{\theta}_{k_{+}+1}, \cdots, \hat{\theta}_{k_{+}+l}\right) \in C^{2 l}, \operatorname{Im} \hat{\theta}_{k_{+}+n} \in\left(0, \frac{1}{2} \mu g\right), \hat{\theta}_{k_{+}+n} \neq \hat{\theta}_{k_{+}+m} \\
& \hat{q}_{k_{+}+l+n}=\overline{\hat{q}}_{k_{+}+n}+i \pi\left[1-(-)^{l}\right] / 2 \mu, \hat{\theta}_{k_{+}+l+n}=\overline{\hat{\theta}}_{k_{+}+n}, n=1, \cdots, l
\end{aligned}
$$

Clearly, any such point belongs to $\mathscr{H}$, cf. (3.7), (3.2). Furthermore, the definitions of the imaginary parts are such that

$$
\eta(\hat{q}, \hat{\theta}) \in N_{l}, \quad(\hat{q}, \hat{\theta}) \in \tilde{\hat{\Omega}}_{l}
$$

where $N_{l}$ is given by (2.44). (This is readily verified from (3.4) and (3.1) by taking the ordering (4.20) of the real $\hat{\theta}_{k}$ into account; cf. also (3.3).)

Second, we may and will identify $\tilde{\Omega}_{l}$ with the above manifold $S_{k_{+}, k_{-}} \times \widetilde{B}_{l}$ by 
setting

$$
\left.\begin{array}{lll}
x_{i}^{s}=\operatorname{Re} \hat{q}_{i}, & p_{i}^{s}=\hat{\theta}_{i}, & i=1, \cdots, k_{+} \\
x_{j}^{\bar{s}}=\operatorname{Re} \hat{q}_{N-k_{-}+j}, & p_{j}^{\bar{s}}=\hat{\theta}_{N-k_{-}+j}, & j=1, \cdots, k_{-} \\
x_{n}=\operatorname{Re} \hat{q}_{k_{+}+n}, & \begin{array}{l}
p_{n}=2 \operatorname{Re} \hat{\theta}_{k_{+}+n} \\
\gamma_{n}=\operatorname{Im} \hat{q}_{k_{+}+n}+\left(k_{+}+n-1\right) \pi / \mu,
\end{array} \delta_{n}=-2 \operatorname{Im} \hat{\theta}_{k_{+}+n}
\end{array}\right\} \quad n=1, \cdots, l .
$$

Then the map $\hat{\pi}_{l}$ in the diagram (4.19) is defined by composing this identification map with the covering projection $\widetilde{B}_{l} \rightarrow B_{l}$, cf. (4.10). Combining (4.1)-(4.3) with (4.20)-(4.26) we now deduce the crucial relations

$$
\begin{aligned}
& v(Q)=T_{\sigma} \eta(\tilde{\hat{P}}) \\
& \tilde{A}(Q)=T_{\sigma} \mathscr{L}(\tilde{\hat{P}}) T_{\sigma}^{t} \\
& \tilde{L}(Q)=T_{\sigma} \mathscr{A}(\tilde{\hat{P}}) T_{\sigma}^{t}
\end{aligned}
$$

where

$$
T_{\sigma} \equiv \operatorname{diag}\left(\mathbf{1}_{k_{+}}, M_{\sigma}, M_{\sigma}, \mathbf{1}_{k_{-}}\right)
$$

and $M_{\sigma} \in O(l)$ is the permutation matrix putting $\hat{\theta}_{k_{+}+1}, \cdots, \hat{\theta}_{k_{+}+l}$ in lexicographic order. (This generalizes (3.17)-(3.19).)

Third, we define the map $Z_{l}$. To this end we first observe that $\tilde{\hat{\Omega}}_{l} \subset \mathscr{H}_{s}$. Indeed, from (2.56) and (2.60), (2.61) we have

$$
\left(\mathscr{M}^{-1} \tilde{A} \mathscr{M}\right)(Q)=\operatorname{diag}\left(\exp \left(\mu x_{1}^{+}\right), \cdots, \exp \left(\mu x_{N_{+}}^{+}\right),-\exp \left(\mu x_{1}^{-}\right), \cdots,-\exp \left(\mu x_{N_{-}}^{-}\right)\right)
$$

and combining this with (4.28) the inclusion is clear. Since the spectrum of $\mathscr{L}$ is real on $\tilde{\hat{\Omega}}_{l}$, we may now invoke Lemmas 3.1 and 3.3 to infer that there exists a branch $\mathscr{B}_{l}$ of $\mathscr{R}$ over $\tilde{\Omega}_{l}$ that is uniquely determined by

$$
\left(\mathscr{B}_{l}^{-1} \mathscr{L} \mathscr{B}_{l}\right)(\tilde{\hat{P}})=\operatorname{diag}\left(\exp \left(\mu x_{1}^{+}\right), \cdots,-\exp \left(\mu x_{N_{-}}^{-}\right)\right) .
$$

By definition, the map $Z_{l}$ is a corresponding branch of $R$ over $\tilde{\hat{\Omega}}_{l}$, uniquely determined by 


$$
q_{j} \equiv\left\{\begin{array}{l}
x_{j}^{+} \\
x_{j-N_{+}}^{-}+i \pi / \mu \\
x_{N_{-}}^{-}+(2 k+1) i \pi / \mu
\end{array}, \quad j=\left\{\begin{array}{l}
1, \cdots, N_{+} \\
N_{+}+1, \cdots, N-1 \\
N
\end{array}\right.\right.
$$

On account of (4.32) and Lemma 3.3 such a branch does occur, with $k \in \mathbb{Z}$ being given by (recall (3.37))

$$
k=\frac{\mu}{2 i \pi}\left[\sum_{k=1}^{N} \hat{q}_{k}-\sum_{i=1}^{N_{+}} x_{i}^{+}-\sum_{j=1}^{N-} x_{j}^{-}\right]-\frac{1}{2} N_{-} .
$$

(Whenever $\tilde{\widehat{\Omega}}_{l}$ has more than one connected component, this integer will depend on the component, cf. (4.21), (4.22).) Moreover, from (4.28) together with (4.31), (4.32) it follows that there exists a diagonal matrix $D$ such that

$$
\mathscr{B}_{l}(\tilde{\hat{P}})=T_{\sigma(\tilde{P})}^{t} \mathscr{M}(Q) D(\tilde{\hat{P}})
$$

(This generalizes (3.23).) Therefore, the map $Z_{l}$ yields a vector $\theta$ given by

$$
\theta_{j}=\left\{\begin{array}{l}
p_{j}^{+} \\
p_{j-N_{+}}^{-}
\end{array} \quad j=\left\{\begin{array}{l}
1, \cdots, N_{+} \\
N_{+}+1, \cdots, N
\end{array}\right.\right.
$$

cf. (4.29) and (2.63), (2.64).

It is now clear how the definition of the diagram (4.19) is to be completed: We set

$$
\tilde{\Omega}_{l} \equiv Z_{l}\left(\tilde{\hat{\Omega}}_{l}\right)
$$

and take for $\pi_{l}$ the obvious identification map

$$
\begin{aligned}
& x_{i}^{+}=q_{i}, \quad p_{i}^{+}=\theta_{i}, \quad i=1, \cdots, N_{+} \\
& x_{j}^{-}=\operatorname{Re} q_{N_{+}+j}, \quad p_{j}^{-}=\theta_{N_{+}+j}, \quad j=1, \cdots, N_{-} \text {. }
\end{aligned}
$$

Then the diagram commutes by construction.

Having detailed the algebraic bottom line, analytic top line and geometric side lines of the diagram (4.19), we can now proceed to the dynamical punch line: from the commutativity relation

$$
\mathscr{E}_{l} \circ \hat{\pi}_{l}=\pi_{l} \circ Z_{l}, \quad \mathscr{E}_{l} \equiv \mathscr{I}_{l} \circ \mathscr{C}_{l}^{-1}
$$


it follows that the bijection $\mathscr{E}_{l}$ is a symplectomorphism onto its range. Indeed, smoothness of $\mathscr{E}_{l}$ is clear from (4.39) and holomorphy of $Z_{l}$, smoothness of $\mathscr{E}_{l}^{-1}$ follows from the fact that $Z_{l}$ has a local holomorphic inverse, and the canonicity of $\mathscr{E}_{l}$ results from (4.33), (4.36) and (3.29) by specializing the holomorphic partials in the latter equations to the relevant directions, cf. (4.20)-(4.24) and (4.26).

Thus far we have concentrated our attention on the submanifold $\Omega_{r}=\Omega_{s} \cap \Omega_{i}$ for which the Lax matrix (2.21) has simple spectrum inside the strip $S_{\mu g / 2}$ cf. (2.28). Of course, for the special case $m \equiv \min \left(N_{+}, N_{-}\right)=0$ this is no restriction, since one then has $\Omega_{r}=\Omega$. However, assuming $m>0$ from now on, $\Omega_{r}$ must be smaller than $\Omega$. Indeed, $\Omega$ is a connected subset of $\boldsymbol{R}^{2 N}$, whereas $\Omega_{r}$ has more than one connected component. On the other hand, $\Omega_{r}$ is only slightly smaller: it is dense in $\Omega$ and has full measure.

To prove the assertion just made, we introduce the exceptional set

$$
\Omega_{e} \equiv \Omega \backslash \Omega_{s}
$$

and note that the complement of $\Omega_{r}$ can be written

$$
\Omega \backslash \Omega_{r}=\Omega_{e} \cup \Omega_{b},
$$

where $\Omega_{b}$ is the boundary set (2.29). Now let $\lambda_{1}, \cdots, \lambda_{N}$ be the roots of the polynomial $\left|L-\lambda \mathbf{1}_{N}\right|$, and define

$$
\begin{aligned}
& D \equiv \prod_{1 \leqslant j<k \leqslant N}\left(\lambda_{j}-\lambda_{k}\right)^{2} \\
& F \equiv \prod_{1 \leqslant j<k \leqslant N}\left[\left(\lambda_{j}-\lambda_{k}\right)^{2}+\mu^{2} g^{2}\right] .
\end{aligned}
$$

Since the functions $D$ and $F$ are invariant under permutations of the $\lambda_{i}$, they must be polynomials in the symmetric functions of $L$. Because $L$ is real-analytic on $\Omega$, it follows that $D$ and $F$ are real-analytic, as well. Now it is clear that $\Omega_{e}$ and $\Omega_{b}$ are just the zero loci of $D$ and $F$, resp., so that these sets are real-analytic subvarieties with codimension at least one. By virtue of (4.41) we may then deduce that $\Omega \backslash \Omega_{r}$ is nowhere dense and has measure zero, implying the assertion.

However, these simple observations leave quite a few questions open that are not as easily answered. In particular, at this stage it is by no means clear whether $\Omega_{e}$ and $\Omega_{b}$ are both non-empty, and what happens to the $\gamma_{n}$-torus when the action variable $\delta_{n}$ converges to $-\mu g$. We shall address such questions in 
Section 5A.

\section{B. The Case $\tilde{I I}_{\text {rel }}$}

When the parameter $\tau=\frac{1}{2} \beta \mu g$ belongs to $(0, \pi / 2)$, we can and will proceed in the same way as in the previous section. Thus, we reparametrize the data set $D$ again via (4.1)-(4.3), with $v_{k}$ replaced by $v_{k} \exp \left(-\beta a_{k}\right)$ and $\mathscr{V}_{k}$ given by (3.57), $k=1, \cdots, N$. (Thus, we get (3.68) in the pure soliton case $N_{-}=0$.) After these substitutions all definitions and relations preceding Theorem 4.1 apply without change.

For $\tau=\pi / 2$ we define the map $\mathscr{C}_{l}$ in the same way as for $\tau \in(0, \pi / 2)$, but now we restrict attention to the subsets

$$
\begin{aligned}
& \hat{\Omega}_{l}^{b} \equiv \mathscr{C}_{l}\left(D_{l}^{b}\right) \\
& \Omega_{l}^{b} \equiv \mathscr{I}\left(D_{l}^{b}\right)
\end{aligned}
$$

of the manifolds $\hat{\Omega}_{l}$ and $\Omega^{b}$, resp. At this point is not yet obvious that any of these sets is a manifold; in fact, thus far we have not even shown that $D_{l}^{b}$ is non-empty. Therefore, we may and will proceed just as for $\tau \in(0, \pi / 2)$, replacing $D$ 's and $\Omega$ 's by $D^{b}$ 's and $\Omega^{\natural}$ 's, but for the moment $\hat{\Omega}_{l}^{b}$ and $\hat{\Omega}^{b}$ can only be viewed as sets. However, in the following theorem these sets are promoted to $2 \mathrm{~N}$ dimensional manifolds, among other things.

Theorem 4.2. For $\tau \in(0, \pi / 2)$ the assertions of Theorem 4.1 hold true. Now let $\tau=\pi / 2$. Then $\hat{\Omega}_{l}^{b}$ is an open dense submanifold of $\hat{\Omega}_{l}, \Omega_{l}^{b}$ is an open submanifold of $\Omega^{b}$, and $\Phi_{l}$ is a symplectomorphism from $\left\langle\Omega_{l}^{b}, \omega\right\rangle$ onto $\left\langle\hat{\Omega}_{l}^{b}, \hat{\omega}_{l}\right\rangle$ with inverse $\mathscr{E}_{l}$. Moreover, $\Phi$ is a symplectomorphism from $\left\langle\Omega_{r}^{b}, \omega\right\rangle$ onto

$$
\left\langle\hat{\Omega}^{b}, \hat{\omega}\right\rangle \equiv \bigcup_{l=0}^{m}\left\langle\hat{\Omega}_{l}^{b}, \hat{\omega}_{l}\right\rangle
$$

with inverse $\mathscr{E}$.

Proof. We first take $\tau \in(0, \pi / 2)$ and follow the reasoning in the proof of Theorem 4.1. From (4.31) and the definition (3.63)-(3.65) of $\mathscr{K}_{s}^{b}$ we deduce $\tilde{\hat{\Omega}}_{l} \subset \mathscr{H}_{s}^{b}$. Then (4.32) again determines a unique branch $\mathscr{B}_{l}$ of $\mathscr{R}$. By definition, the map $Z_{l}$ is again a corresponding branch of $R$. We may and will require that the vector $q$ is given by (4.33), but since we have not determined the 
$\theta$-monodromy, it is not clear whether this requirement fixes a unique branch of $R$. (Most likely, it does not, cf. the paragraph preceding Lemma 3.6.) However, $\mathscr{B}_{1}(\tilde{\hat{P}})$ must again be related to $\mathscr{M}(Q)$ via (4.35), so that any branch corresponding to $\mathscr{B}_{l}$ yields a vector $\theta$ of the form

$$
\theta_{j}=\left\{\begin{array}{l}
p_{j}^{+}+i \pi n_{j} / \beta \\
p_{j-N_{+}}^{-}+i \pi n_{j} / \beta
\end{array} \quad j=\left\{\begin{array}{l}
1, \cdots, N_{+} \\
N_{+}+1, \cdots, N
\end{array}\right.\right.
$$

where $n_{j} \in Z$. Then a unique branch can be fixed by elimination. (For instance, one can proceed as follows: discard branches for which $n_{1} \neq 0$ except if no branch remains; in the latter case discard branches whose $\left|n_{1}\right|$ is larger than the minimum $\left|n_{1}\right|$; then discard branches whose $n_{1}$ is negative except if no branch remains. Repeating this procedure for $n_{2}, \cdots, n_{N-1}$, yields a unique set of integers $n_{1}, \cdots, n_{N}\left(n_{N}\right.$ being determined by $\Sigma n_{j}=0$, cf. (3.76)), and hence a unique branch $Z_{l}$.) To complete the definition of the diagram (4.19), we now define $\Omega_{l}$ by (4.37) and $\pi_{l}$ by (4.38) with $\theta_{k}$ replaced by $\operatorname{Re} \theta_{k}$. Then the diagram again commutes by construction, and the proof can be finished as before.

Now let $\tau=\pi / 2$. Then we can once more construct a commutative diagram: In (4.19) we replace the set $D_{l}$ by $D_{l}^{b}$ and the four $\Omega$ 's by $\Omega^{b}$ 's. The bottom line has already been defined, so we may proceed by setting

$$
\widetilde{\hat{\Omega}}_{l}^{b} \equiv \tilde{\hat{\Omega}}_{l} \backslash \mathscr{N}_{\mathscr{F}}
$$

Here, $\tilde{\hat{\Omega}}_{l}$ is defined as before, and $\mathscr{N}_{\mathscr{F}}$ is defined by (3.63), (3.64). We claim that $\tilde{\hat{\Omega}}_{l}^{b}$ is a (relatively) open dense subset of $\tilde{\hat{\Omega}}_{l}$. This claim will be justified shortly. Since the function $\mathscr{F}$ is invariant under the $\left(Z^{l} \rtimes S_{l}\right)$-action, we may compose the identification map (4.26) restricted to $\tilde{\Omega}_{l}^{b}$ with the covering projection $\tilde{B}_{l} \rightarrow B_{l}$. By definition, this yields the map $\hat{\pi}_{l}$, and now it also follows that $\hat{\Omega}_{l}^{b}$ is an open dense submanifold of $\hat{\Omega}_{l}$. Hence $D_{l}^{b}$ is an open dense subset of $D_{l}$ and $\Omega_{l}^{b}$ is non-empty. Now $\Omega_{l}^{b}$ is an open set (as is clear from its definition), so from here on our previous arguments apply. Therefore, we are left with proving the claim.

Clearly, it suffices to show that each connected component of $\tilde{\Omega}_{l}$ contains points that do not belong to $\mathscr{N}_{\mathscr{F}}$. This can be seen as follows. Fixing any interlacing in (4.20), we may choose $z \equiv \hat{\theta}(\hat{q})$ such that the real parts of $z_{1}, \cdots, z_{k_{+}+l}, z_{N-k_{-}+1}, \cdots, z_{N}$ are distinct and a minimal distance $\mu g \Lambda(R)$ apart and such that $\operatorname{Im} z_{k_{+}+n}$ equals $\mu g / 4(\pi / 2 \mu)$, cf. (4.23), (4.24). Then it is straightforward to verify that the limit $\mathscr{L}(\infty)$ of $\mathscr{L}(\Lambda) \equiv \mathscr{L}(\hat{q}, \hat{\theta})$ for $\Lambda \rightarrow \infty$ exists and that $\mathscr{L}(\infty)$ has eigenvalues $\alpha_{1}(\infty), \cdots, \alpha_{N}(\infty)$ such that $\alpha_{i}(\infty)+\alpha_{j}(\infty) \neq 0$, provided $R$ 
is chosen large enough. Fixing such an $R$, it follows from a perturbation argument exemplified by (3.52) that the eigenvalues of $\mathscr{L}(\Lambda)$ satisfy $\alpha_{i}(\Lambda)+$ $\alpha_{j}(\Lambda) \neq 0$ for $\Lambda$ large enough.

For $\tau \in(0, \pi / 2)$ one infers just as in the $\tilde{\mathrm{I}}_{\mathrm{nr}}$ case that $\Omega_{r}$ is a dense subset of $\Omega$ with full measure: One need only replace $(4.43)$ by

$$
F \equiv \prod_{1 \leqslant j<k \leqslant N}\left[\left(\lambda_{j}-\lambda_{k}\right)^{2}+4 \lambda_{j} \lambda_{k} \sin ^{2} \tau\right]
$$

where $\lambda_{1}, \cdots, \lambda_{N}$ are the roots of the characteristic polynomial of the Lax matrix (2.70), and follow the reasoning after Theorem 4.1.

For $\tau=\pi / 2$ we should compare $\Omega_{r}^{b}$ and $\Omega^{b}$. Since $\Omega^{b}$ is the subset of $\Omega$ on which $x_{i}^{+} \neq x_{j}^{-}, \quad i=1, \cdots, N_{+}, \quad j=1, \cdots, N_{-}$, it has $N ! / N_{+} ! N_{-} ! \equiv c$ connected components. Likewise, $\hat{\Omega}_{0} \simeq S_{N_{+}, N_{-}}$has $c$ components (cf. (4.6)), so that $\hat{\Omega}_{0}^{b}$ has at least $c$ components. Now $\hat{\Omega}_{0}^{b}$ is a proper open subset of $\hat{\Omega}^{b}$, so that $\hat{\Omega}^{b}$ has more than $c$ components. But then $\Omega_{r}^{b}$ has more components than $\Omega^{b}$, since $\hat{\Omega}^{b}$ and $\Omega_{r}^{b}$ are homeomorphic by virtue of the theorem just proved. Therefore, $\Omega^{b} \backslash \Omega_{r}^{b}$ is a codimension-one real-analytic subvariety of $\Omega^{b}$, and as such is nowhere dense and has measure zero. In Section 5B we shall see that only the discriminant function $D$ vanishes on $\Omega^{b} \backslash \Omega_{r}^{b}$; the function $F$ is non-zero on $\Omega^{b}$, so that $\Omega_{r}^{b}=\Omega_{s}^{b}$.

\section{The Harmonic Oscillator Transform}

\section{A. The Case $\tilde{\mathbf{I}}_{\mathrm{nr}}$}

As promised at the end of Section 4A, we shall now obtain more information on the sets $\Omega_{b}$ and $\Omega_{e}$, and clarify the behavior of the $\gamma_{n}$-tori as one or more of the actions $\delta_{n}$ converge to $-\mu g$. To provide background, intuition and notation for the general case, we begin by studying the case $N_{+}=N_{-}=1$, where virtually any question can easily be answered explicitly. In particular, one finds that $\Omega_{e}$ is a codimension-one submanifold on which $L$ is not diagonalizable, and that $\Omega_{b}$ is a codimension-two submanifold; specifically,

$$
\begin{aligned}
& \Omega_{e}=\left\{\left(x^{+}, x^{-}, p^{+}, p^{-}\right) \in \boldsymbol{R}^{4} \mid\left(p^{+}-p^{-}\right)^{2}=\mu^{2} g^{2} / \mathrm{ch}^{2} \frac{1}{2} \mu\left(x^{+}-x^{-}\right)\right\} \\
& \Omega_{b}=\left\{\left(x^{+}, x^{-}, p^{+}, p^{-}\right) \in \boldsymbol{R}^{4} \mid x^{+}=x^{-}, p^{+}=p^{-}\right\}
\end{aligned}
$$


Thus, $\Omega_{e}$ consists of two disjoint hypersurfaces separating the two components of the soliton manifold $S_{1,1}$ from the breather manifold $B_{1}$ (recall (4.6), (4.7)), and $\Omega_{b}$ consists of the 'dead breather' points.

Of course, in the case at hand it is evident from a phase space picture that the $\gamma$-torus collapses to a point when the action variable $\delta$ converges to $-\mu g$. To be more precise, let us introduce the 'harmonic oscillator variables'.

$$
\begin{gathered}
u \equiv(2 g)^{1 / 2}(1+\delta / \mu g)^{1 / 2} \sin \mu \gamma \quad \gamma=\frac{1}{\mu} \operatorname{arctg}(u / v) \\
\Leftrightarrow \\
v \equiv(2 g)^{1 / 2}(1+\delta / \mu g)^{1 / 2} \cos \mu \gamma \quad \delta=\frac{1}{2} \mu\left(u^{2}+v^{2}-2 g\right)
\end{gathered}
$$

and observe that this transformation densely embeds $B_{1}$ into an extended breather manifold

$$
B_{1}^{\sharp} \equiv\left\{(x, u, p, v) \in \boldsymbol{R}^{4} \mid u^{2}+v^{2}<2 \mathrm{~g}\right\} .
$$

Let us now compose the action-angle transform $\Phi_{1}$ from $\Omega_{1}$ onto $\hat{\Omega}_{1}=B_{1}$ with the coordinate change (5.3) and denote the resulting diffeomorphism again by $\Phi_{1}$. Then one readily verifies that $\Phi_{1}$ can be extended to a "harmonic oscillator transform' $\Phi_{1}^{\#}$ from $\Omega_{1}^{\#} \equiv \Omega_{1} \cup \Omega_{b}$ onto $\tilde{\Omega}_{1}^{\#} \equiv B_{1}^{\#}$. Explicitly, one finds that $\Phi_{1}^{\#}$ is given by

$$
\begin{aligned}
& x=\frac{1}{2}\left(x^{+}+x^{-}\right), \quad p=p^{+}+p^{-} \\
& u=-\frac{\delta}{\mu}\left(\frac{2}{g}\right)^{1 / 2}\left(1-\frac{\delta}{\mu g}\right)^{-1 / 2} \operatorname{sh} \frac{1}{2} \mu\left(x^{+}-x^{-}\right) \\
& v=\frac{1}{\mu}\left(\frac{2}{g}\right)^{1 / 2}\left(1-\frac{\delta}{\mu g}\right)^{-1 / 2}\left(p^{+}-p^{-}\right) \operatorname{ch} \frac{1}{2} \mu\left(x^{+}-x^{-}\right) \\
& \delta=-\left[\frac{\mu^{2} g^{2}}{\operatorname{ch}^{2} \frac{1}{2} \mu\left(x^{+}-x^{-}\right)}-\left(p^{+}-p^{-}\right)^{2}\right]^{1 / 2},
\end{aligned}
$$

whereas the inverse transformation $\mathscr{E}_{1}^{\#}$ reads 


$$
\begin{aligned}
& x^{ \pm}=x \pm \frac{1}{\mu} \operatorname{Arth}(u r) \\
& p^{ \pm}=\frac{1}{2} p \pm \frac{1}{4} \mu v r\left(2 g-u^{2}-v^{2}\right),
\end{aligned}
$$

where

$$
r \equiv\left[\frac{4 g-u^{2}-v^{2}}{4 g^{2}-4 g v^{2}+u^{2} v^{2}+v^{4}}\right]^{1 / 2}
$$

Moreover, equipping $\hat{\Omega}_{1}^{\sharp}$ with the symplectic form

$$
\hat{\omega}_{1}^{\#} \equiv d x \wedge d p+d u \wedge d v,
$$

the maps $\Phi_{1}^{\#}$ and $\mathscr{E}_{1}^{\sharp}$ are canonical transformations. Finally, we note that (5.6)-(5.8) entail

$$
\lim _{\delta \uparrow 0} u=0, \quad \lim _{\delta \uparrow 0} v=(2 g)^{1 / 2} \epsilon\left(p^{+}-p^{-}\right) \quad\left(p^{+}-p^{-} \text {fixed }\right) .
$$

It would be overly greedy to expect such explicitness for $N>2$. Even so, the main questions can again be qualitatively answered, as will now be detailed. First, let us present a quite direct argument showing $\Omega_{b}$ is not empty. (We shall greatly improve on this result in Theorem 5.1, but this involves a lot more preparation; the a priori bounds in the argument that follows will be needed again.)

We start from the symmetric functions of $\mathscr{L}$ on its definition domain $\mathscr{H}$. Using (3.1)-(3.5) and (B4) one verifies that these are given by

$$
S_{k}(\hat{q}, \hat{\theta})=\sum_{\substack{I \subset\{1, \cdots, N\} \\|I|=k}} \exp \left(\mu \sum_{i \in I} \hat{q}_{i}\right) \prod_{\substack{i \in I \\ j \notin I}}\left[\left(\hat{\theta}_{i}-\hat{\theta}_{j}\right)^{2}+\mu^{2} g^{2}\right]^{1 / 2} /\left(\hat{\theta}_{\min (i, j)}-\hat{\theta}_{\max (i, j)}\right)
$$

Now we specialize $(\hat{q}, \hat{\theta})$ to the covering manifold $\tilde{\hat{\Omega}}_{l}$ of $\hat{\Omega}_{l}($ defined by (4.20)-(4.24)) and kill the $n^{\text {th }}$ breather by taking $\operatorname{Im} \hat{\theta}_{k_{+}+n} \uparrow \frac{1}{2} \mu g$ (and, therefore, $\operatorname{Im} \hat{\theta}_{k_{+}+l+n} \downarrow-$ $\frac{1}{2} \mu g$ ). Using $\operatorname{Re} \hat{\theta}_{k_{+}+n}=\operatorname{Re} \hat{\theta}_{k_{+}+l+n}$ one reads off from (5.13) that $S_{k}$ has a limit to which only subsets $I$ contribute that either contain both indices $k_{+}+n$, 
$k_{+}+l+n$ or contain neither of the two. (Note the limit does not depend on $\operatorname{Im} \hat{q}_{k_{+}+n}$, cf. (4.24).)

More generally, we can kill as many breathers as we please, as long as we ensure that the real parts of the $\hat{\theta}_{k_{+}+n}$ involved are distinct. Since the functions $S_{1}, \cdots, S_{N}$ converge, the eigenvalues of $\mathscr{L}$ have limits, too. Moreover, none of the latter limits can be zero, since $|\mathscr{L}|=S_{N} \neq 0$. Therefore, the corresponding position vectors $x^{+}$and $x^{-}$converge as well.

However, the position limits might not belong to $G_{N_{+}}$and $G_{N_{-}}$, resp. Put differently, some points in $\sigma(\mathscr{L}(\hat{q}, \hat{\theta}))$ might collide, a priori. To exclude this contingency, we first observe that

$$
\frac{1}{2} \operatorname{Tr} \mathscr{A}(\tilde{\hat{P}})^{2}=\frac{1}{2} \operatorname{Tr} L(P)^{2}=H(P)
$$

cf. (4.19), (4.29), (2.23). Next, we note the lhs converges, so that $H$ has a limit, too. But from (1.1) it is then plain that there is a non-zero lower bound on the distances $\left|x_{i}^{\delta}-x_{j}^{\delta}\right|, \delta=+,-$, so no collisions can occur. Now (1.1) also entails that $p^{+}$and $p^{-}$remain bounded. Via a compactness argument it then follows that one can find convergent subsequences. Thus $\Omega_{b}$ is non-empty, as advertised.

Next, we are headed for a complete elucidation of the structure of $\Omega_{b} \cap \Omega_{s}$ (entailing in particular that $p^{+}$and $p^{-}$actually converge in the limit just studied). To this end we introduce an extended breather manifold $B_{l}^{\sharp}$ by trading $\gamma_{n}, \delta_{n}$ for $u_{n}, v_{n}$, cf. (5.3), and by setting

$$
\begin{array}{r}
B_{l}^{\#} \equiv\left\{(x, u, p, v) \in \boldsymbol{R}^{4 l} \mid u_{n}^{2}+v_{n}^{2}<2 g, p_{n}-i \delta_{n} \neq p_{k}-i \delta_{k},\right. \\
\left.p_{1}-i \delta_{1}, \cdots, p_{l}-i \delta_{l} \text { in lexicographic order }\right\} \\
\delta_{n} \equiv \frac{1}{2} \mu\left(u_{n}^{2}+v_{n}^{2}-2 g\right), \quad u_{n}^{2}+v_{n}^{2}<2 g, \quad n=1, \cdots, l
\end{array}
$$

(recall (4.7)). More precisely, one obtains a manifold $B_{l}^{\sharp c}$ when one omits the ordering restriction in (5.15), and then $B_{l}^{\sharp}$ can and will be viewed as

$$
B_{l}^{\#} \equiv B_{l}^{\# c} / S_{l} .
$$

We now introduce symplectic manifolds $\left\langle\hat{\Omega}_{l}^{\sharp(c)}, \hat{\omega}_{l}^{\sharp(c)}\right\rangle$ by

$$
\hat{\Omega}_{l}^{\#(c)} \equiv S_{k_{+}, k_{-}} \times B_{l}^{\#(c)}
$$




$$
\hat{\omega}_{l}^{\sharp(c)} \equiv \sum_{i=1}^{k_{+}} d x_{i}^{s} \wedge d p_{i}^{s}+\sum_{j=1}^{k_{-}} d x_{j}^{\bar{s}} \wedge d p_{j}^{\bar{s}}+\sum_{n=1}^{l}\left(d x_{n} \wedge d p_{n}+d u_{n} \wedge d v_{n}\right)
$$

(recall (4.5), (4.11)), and then set

$$
\left\langle\hat{\Omega}^{\sharp}, \hat{\omega}^{\sharp}\right\rangle \equiv \bigcup_{l=0}^{m}\left\langle\hat{\Omega}_{l}^{\#}, \hat{\omega}_{l}^{\sharp}\right\rangle .
$$

Moreover, we denote by $\Omega_{l}^{\sharp}$ the subset of $\Omega_{s}$ on which $L$ has $l$ (and only $l$ ) complex-conjugate pairs of eigenvalues. Thus we have

$$
\Omega_{s}=\bigcup_{l=0}^{m} \Omega_{l}^{\#} .
$$

We are now prepared for the principal result of this section.

Theorem 5.1. The maps $\Phi_{l}$ and $\Phi$ extend to symplectomorphisms $\Phi_{l}^{\#}$ and $\Phi^{\sharp}$ from $\left\langle\Omega_{l}^{\#}, \omega\right\rangle$ onto $\left\langle\hat{\Omega}_{l}^{\#}, \hat{\omega}_{l}^{\#}\right\rangle$ and from $\left\langle\Omega_{s}, \omega\right\rangle$ onto $\left\langle\hat{\Omega}^{\#}, \hat{\omega}^{\#}\right\rangle$, resp. The sets $\Omega_{e}$ and $\Omega_{b} \cap \Omega_{s}$ have codimension one and two, resp., and $L$ is not diagonalizable on $\Omega_{e}$.

Proof. In order to prove the existence of smooth extensions we are going to make use of notation and arguments from the proofs of Theorem 4.1 and Lemma 3.1. We begin by identifying $\tilde{\hat{\Omega}}_{l}$ with the manifold $S_{k_{+}, k_{-}} \times \widetilde{B}_{l} \subset \boldsymbol{R}^{2 N}$ via the identification map (4.26). Then the commutativity of the diagram (4.19) entails that $\mathscr{B}_{l}$ and $Z_{l}$ give rise to real-analytic functions of $\left(x^{s}, x^{\bar{s}}, p^{s}, p^{\bar{s}} ; x, \gamma, p, \delta\right)$ that are invariant under the $Z^{l}$-action (4.9) on $\tilde{B}_{l}$. Therefore, $\mathscr{B}_{l}$ and $Z_{l}$ descend to real-analytic functions (again denoted $\mathscr{B}_{l}, Z_{l}$ ) on

$$
\hat{\Omega}_{l}^{c} \equiv S_{k_{+}, k_{-}} \times B_{l}^{c}, \quad B_{l}^{c} \equiv \widetilde{B}_{l} / Z^{l} .
$$

We may and will view $B_{l}^{c}$ as the open subset of $\boldsymbol{R}^{4 l}$ obtained via the transformations $\gamma_{n}, \delta_{n} \mapsto u_{n}, v_{n}, n=1, \cdots, l$, cf. (5.3). Explicitly, this amounts to

$$
B_{l}^{c} \equiv\left\{(x, u, p, v) \in \boldsymbol{R}^{4 l} \mid 0<u_{n}^{2}+v_{n}^{2}<2 g, p_{n}-i \delta_{n} \neq p_{k}-i \delta_{k}\right\} .
$$

Correspondingly, we may and will view $\hat{\Omega}_{l}^{c}$ as the open dense submanifold obtained from $\hat{\Omega}_{l}^{\# c}$ by requiring $\left(u_{n}, v_{n}\right) \neq(0,0), n=1, \cdots, l$.

As a result of these considerations, we may now replace (4.19) by the commutative diagram 


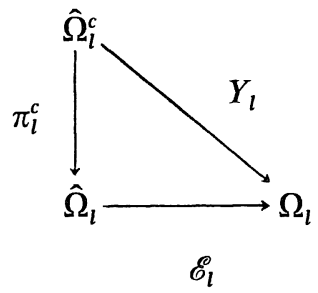

Here, the map

$$
Y_{l} \equiv \pi_{l} \circ Z_{l}
$$

is real-analytic and symplectic, and invariant under the $S_{l}$-action on the breather variables $(x, u, p, v)$. Moreover, $\pi_{l}^{c}$ is the covering projection that quotients out the $S_{l}$-action, and just as before we abuse notation by denoting the images of $\mathscr{E}_{l}$ and $\hat{\Omega}_{l}$ under the canonical coordinate change $(\gamma, \delta) \mapsto(u, v)$ by the same symbols.

We are now aiming for an extension of (5.24) to the following diagram:

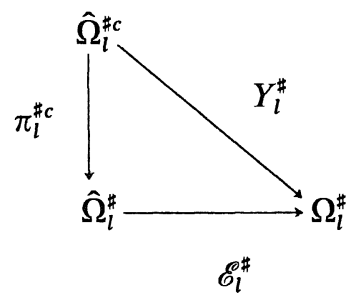

Here, the manifolds $\hat{\Omega}_{l}^{\#(c)}$ and $\Omega_{l}^{\#}$ have been defined already (recall (5.18), (5.21)) and $\pi_{l}^{\# c}$ is the covering projection extending $\pi_{l}^{c}$. Thus, the definition of the diagram will be complete once we define the maps $Y_{l}^{\sharp}$ and $\mathscr{E}_{l}^{\#}$.

We proceed by doing so. The result will be that $Y_{l}^{\#}$ is a real-analytic symplectic map from $\hat{\Omega}_{l}^{\# c}$ into $\Omega_{l}^{\#}$ that extends $Y_{l}$, whereas $\mathscr{E}_{l}^{\#}$ is a bijection from $\hat{\Omega}_{l}^{\#}$ onto $\Omega_{l}^{\#}$ that extends $\mathscr{E}_{l}$. The diagram will commute by construction, so that

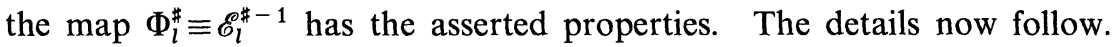

First, we restrict the vector-valued function $\eta$ and matrix-valued function $\mathscr{L}$ from Section $3 \mathrm{~A}$ to $\tilde{\Omega}_{l} \simeq S_{k_{+}, k_{-}} \times \widetilde{B}_{l}$ and trade $(\gamma, \delta)$ for $(u, v)$. Then we obtain real-analytic functions (denoted by the same symbols) on $\hat{\Omega}_{l}^{c}$. Now one readily deduces from (3.4), (4.26) and (5.3) that $\eta$ has a real-analytic extension to $\hat{\Omega}_{l}^{\sharp c}$, whereas (3.5) and (B1) entail that the matrix element $\mathscr{L}_{k_{+}+n, k_{+}+l+n}$ diverges when $\left(u_{n}, v_{n}\right)$ converges to $(0,0)$. Therefore, one should not expect that the matrix-valued function $\mathscr{B}_{l}$ occurring in the proof of Theorem 4.1 has a 
real-analytic extension (and indeed it does not, as we shall presently see).

To cope with this problem we introduce a renormalized matrix

$$
\mathscr{L}_{r} \equiv D_{r} \mathscr{L} D_{r}^{-1}
$$

were

$$
D_{r} \equiv \operatorname{diag}\left(\mathbb{1}_{k_{+}}, \eta_{k_{+}+1}, \cdots, \eta_{k_{+}+l}, \mathbb{1}_{N_{-}}\right) .
$$

The point is, that the singularity in the matrix element $C_{j k}$ arising for $\left(u_{n}, v_{n}\right) \rightarrow(0,0)$ is then canceled by zeros coming from $\eta_{j}$ and $\eta_{k}$, where $j \equiv k_{+}+n, k \equiv k_{+}+l+n$. Indeed, a straightforward calculation yields

$$
\begin{aligned}
& \left(\mathscr{L}_{r}\right)_{j k}=\exp \left(2 \mu x_{n}\right) \mu g\left(2 \operatorname{Im} a_{j}+\mu g\right)\left(2 \operatorname{Im} a_{j}\right)^{-2} \\
& \cdot \prod_{i \neq j, k} \frac{\left[\left(a_{j}-a_{i}\right)^{2}+\mu^{2} g^{2}\right]^{1 / 2}}{\left(a_{j}-a_{i}\right)} \cdot \frac{\left[\left(a_{k}-a_{i}\right)^{2}+\mu^{2} g^{2}\right]^{1 / 2}}{\left(a_{k}-a_{i}\right)} \\
& j=k_{+}+n, k=j+l, n=1, \cdots, l .
\end{aligned}
$$

(Here, we have used the notation

$$
a \equiv\left(p^{s}, \frac{1}{2}(p-i \delta), \frac{1}{2}(p+i \delta), p^{\bar{s}}\right)
$$

and $\delta$ is to be viewed as a function of $u$ and $v, \mathrm{cf}$ (5.16)). Therefore, $\mathscr{L}_{r}$ does have a real-analytic extension to $\hat{\Omega}_{l}^{\sharp c}$. Furthermore, the renormalized vectors

$$
\zeta_{r} \equiv D_{r} \zeta, \quad \eta_{r} \equiv D_{r}^{-1} \eta
$$

have real-analytic extensions, as well.

The crux is now, that $\mathscr{L}_{r}$ is not only real-analytic, but also has real and simple spectrum on $\hat{\Omega}_{l}^{\sharp c}$. (Indeed, we know already this holds true on the dense submanifold $\hat{\Omega}_{l}^{c}$. Taking limits, reality is obviously preserved, and no collisions can occur by virtue of the argument in the paragraph containing (5.14).) Consequently, we may mimic the reasoning in the proof of Lemma 3.1 to infer that there exists a real-analytic function $\mathscr{B}_{l, r}$ from $\hat{\Omega}_{l}^{\# c}$ to $G L(N, C)$ satisfying

$$
\begin{aligned}
& \left(\mathscr{B}_{l, r}^{-1} \mathscr{L}_{r} \mathscr{B}_{l, r}\right)_{j k}=0, \quad j \neq k \\
& \mathscr{B}_{l, r}^{-1} \zeta_{r}=\zeta .
\end{aligned}
$$


Moreover, this function is unique up to right multiplication by a permutation matrix. We can and will fix this ambiguity by requiring

$$
\mathscr{B}_{l, \mathrm{r}}=D_{r} \mathscr{B}_{l} \quad \text { (on } \hat{\Omega}_{l}^{c} \text { ). }
$$

(Since $\mathscr{B}_{l, r}$ is invertible on $\hat{\Omega}_{l}^{\# c}$ and $\left|D_{r}\right|=0$ when one or more $\left(u_{n}, v_{n}\right)$ equal $(0,0)$, one can now deduce that $\mathscr{B}_{l}$ does not admit an extension, as asserted above.)

We now define $Y_{l}^{\#}$ in the same way as $Y_{l}$, except that we use $\mathscr{L}_{r}$ and $\mathscr{B}_{l, r}$. Since the matrices entering into this definition are all real-analytic on $\hat{\Omega}_{l}^{\sharp c}$, the map $Y_{l}^{\#}$ is real-analytic. Moreover, since $\mathscr{A}$ is similar to $L, Y_{l}^{\#}$ maps into $\Omega_{l}^{\#}$, as promised. Finally, since $Y_{l}^{\sharp}$ coincides with $Y_{l}$ on $\hat{\Omega}_{l}^{c}, Y_{l}^{\#}$ inherits the salient properties of $Y_{l}$, on account of real-analyticity and the denseness of $\hat{\Omega}_{l}^{c}$ in $\hat{\Omega}_{l}^{\sharp c}$; specifically, $Y_{l}^{\#}$ is symplectic and invariant under the $S_{l}$-action on $B_{l}^{\sharp c}$.

We are now in the position to define $\mathscr{E}_{l}^{\#}$ and hence to complete the definition of the diagram (5.26). To this end, let $\hat{P} \in \hat{\Omega}_{l}^{\sharp}$ and let $\hat{P}^{c} \in \pi_{l}^{\sharp c-1}(\hat{P})$. Then we set $\mathscr{E}_{l}^{\sharp}(\hat{P}) \equiv Y_{l}^{\sharp}\left(\hat{P}^{c}\right)$. (This is well defined, since $Y_{l}^{\sharp}$ is $S_{l}$-invariant.) As a consequence, the diagram commutes and $\mathscr{E}_{l}^{\#}$ is a smooth symplectic extension of the symplectomorphism $\mathscr{E}_{l}$. Therefore, it remains to show that $\mathscr{E}_{l}^{\#}$ is a bijection onto $\Omega_{l}^{\#}$. To this end we shall construct a map $\varphi_{l}: \Omega_{l}^{\#} \rightarrow \hat{\Omega}_{l}^{\#}$ satisfying

$$
\begin{gathered}
\mathscr{E}_{l}^{\sharp} \circ \varphi_{l}=\mathrm{id}_{\Omega_{l}^{\sharp}} \\
\varphi_{l} \circ \mathscr{E}_{l}^{\sharp}=\mathrm{id}_{\Omega_{l}^{\sharp}} .
\end{gathered}
$$

(Once we succeed in doing so, we have $\Phi_{l}^{\sharp}=\varphi_{l}$, of course.)

As a preparation for the definition of $\varphi_{l}$ we choose a point $P \in \Omega_{l}^{\#}$ and diagonalize $L(P)$ with

$$
\mathscr{P}_{r} \equiv E_{r}^{-1} K U^{-1}
$$

where

$$
E_{r} \equiv \operatorname{diag}\left(\hat{e}_{1}, \cdots, \hat{e}_{k_{+}}, 1 / \tilde{e}_{k_{+}+1}, \cdots, 1 / \tilde{e}_{k_{+}+l}, \hat{e}_{k_{+}+l+1}, \cdots, \hat{e}_{N}\right)
$$

Here we are using the matrices $U, K$ and vectors $\hat{e}, \tilde{e}$ from Sections $2 \mathrm{~A}$ and 2B. (To verify that the components of $\hat{e}$ and $\tilde{e}$ entering $E_{r}$ are non-zero one need only recall (2.18) and (2.24). Note that $E$ is not invertible when $P \in \Omega_{l}^{\sharp} \backslash \Omega_{l}$, cf. (2.31), (2.34).) It is easy to see that $\mathscr{P}_{r}$ is the unique matrix such that

$$
\mathscr{P}_{r} L \mathscr{P}_{r}^{-1}=\operatorname{diag}\left(a_{1}, \cdots, a_{N}\right), \quad a \in A_{l}^{\#}
$$

where 


$$
A_{l}^{\#} \equiv \Lambda_{l} \cap\left(S_{\mu g / 2}^{c l}\right)^{N}
$$

(cf. (2.4) and (2.43)) and such that

$$
\begin{array}{ll}
\left(\mathscr{P}_{r} e\right)_{j}=1, & j \neq k_{+}+1, \cdots, k_{+}+l \\
\left(\mathscr{P}_{r}^{-1 t} e\right)_{j}=1, & j=k_{+}+1, \cdots, k_{+}+l .
\end{array}
$$

Next, we note that

$$
\begin{array}{ll}
\left(\mathscr{P}_{r} e\right)_{j}=v_{j}, & j=k_{+}+1, \cdots, k_{+}+l \\
\left(\mathscr{P}_{r}^{-1 t} e\right)_{j}=v_{j}, & j \neq k_{+}+1, \cdots, k_{+}+l
\end{array}
$$

where $v$ is defined by (2.40). Now it follows just as in the special case $P \in \Omega_{l}$ that (2.42) holds true, except that $C^{*}$ should be replaced by $C$. Moreover, setting

$$
\tilde{A}_{r} \equiv \mathscr{P}_{r} A \mathscr{P}_{r}^{-1}
$$

we deduce

$$
\left(\tilde{A}_{r}\right)_{j k}=\tilde{e}_{j} \hat{A}_{j k} \hat{e}_{k}>0, \quad j=k_{+}+n, \quad k=j+l, \quad n=1, \cdots, l
$$

by using (2.18) and (2.16).

We are finally in the position to specify $\varphi_{l}$. First, we define vectors $x^{s} \in \boldsymbol{R}^{k_{+}}$, $x^{\bar{s}} \in \boldsymbol{R}^{k-}$ by setting

$$
\begin{aligned}
& v_{i} \equiv \exp \left(\mu x_{i}^{s}\right)\left|\mathscr{V}_{i}(a)\right|, \quad i=1, \cdots, k_{+} \\
& v_{N-k_{-}+j} \equiv-\exp \left(\mu x_{j}^{\bar{s}}\right)\left|\mathscr{V}_{N-k_{-}+j}(a)\right|, \quad j=1, \cdots, k_{-} .
\end{aligned}
$$

Second, we define a vector $x \in \boldsymbol{R}^{l}$ by parametrizing $\left(\tilde{A}_{r}\right)_{k_{+}+n, k_{+}+l+n}$ via the $r h s$ of (5.29). (In view of (2.42) and (5.44) all positions can indeed be chosen real.) Third, we define $u, v \in \boldsymbol{R}^{l}$ by writing

$$
\begin{aligned}
v_{j} \equiv & (-)^{j-1} \exp \left(\mu x_{n}\right)\left(u_{n}-i v_{n}\right)(\mu / 2)^{1 / 2}\left(2 \operatorname{Im} a_{j}+\mu g\right)^{1 / 2}\left(2 \operatorname{Im} a_{j}\right)^{-1} \\
& \cdot \prod_{i \neq j, j+l} \frac{\left[\left(a_{j}-a_{i}\right)^{2}+\mu^{2} g^{2}\right]^{1 / 2}}{a_{j}-a_{i}}, \quad j=k_{+}+n, \quad n=1, \cdots, l .
\end{aligned}
$$

Fourth, we parametrize the vector $a \in A_{l}^{\#}$ by using (5.30), which yields $p^{s} \in G_{k_{+}}$, 
$p^{\bar{s}} \in G_{k_{-}}, p \in \boldsymbol{R}^{l}$ and $\delta \in[-\mu g, 0)^{l}$.

Setting now

$$
\varphi_{l}(P) \equiv\left(x^{s}, x^{\bar{s}}, p^{s}, p^{\bar{s}} ; x, u, p, v\right), \quad P \in \Omega_{l}^{\#}
$$

we claim that the rhs belongs to $\hat{\Omega}_{l}^{\sharp}$. To prove this, we need only show that $\delta_{n}$ is related to $u_{n}, v_{n}$ via (5.16), all other properties then being clear. To this purpose we observe that on the one hand the parametrizations just detailed imply

$$
\hat{e}_{j} \tilde{e}_{k} / \hat{A}_{j k}=v_{j} v_{k} /\left(\tilde{A}_{r}\right)_{j k}=\left(u_{n}^{2}+v_{n}^{2}\right) / 2 g, \quad j=k_{+}+n, \quad k=j+l .
$$

(Recall also (5.44) and (2.42) to verify this.) On the other hand, we infer from (2.24) that

$$
\hat{e}_{j} \tilde{e}_{k} / \hat{A}_{j k}=1+\delta_{n} / \mu g, \quad j=k_{n}+n, \quad k=j+l
$$

so that the desired relation (5.16) results.

It remains to prove that $\varphi_{l}$ satisfies (5.35) and (5.36). To this end we identify $\varphi_{l}(P)$ with a point $\hat{P}^{c} \in \hat{\Omega}_{l}^{\# c}$ in the obvious way (namely, by keeping the lexicographic ordering). Then we must have

$$
\mathscr{B}_{l, r}\left(\hat{P}^{c}\right)=\mathscr{P}_{r}(P) \operatorname{diag}\left(e_{1}(P), \cdots, e_{N}(P)\right)
$$

since the matrix at the $r h s$ has the properties that uniquely determine $\mathscr{B}_{l, r}\left(\hat{P}^{c}\right)$. From this (5.35) is clear. Conversely, picking $\hat{P} \in \hat{\Omega}_{l}^{\#}$ and identifying $\hat{P}$ with $\hat{P}^{c} \in \hat{\Omega}_{l}^{\sharp c}$, we obtain an image point $P \in \Omega_{l}^{\#}$ whose $\mathscr{P}_{r}$ must satisfy (5.50), again by uniqueness. Thus, (5.36) follows and the proof of the first assertion of the theorem is complete.

To prove the assertions concerning codimension, we note that $\Omega$ is connected, whereas $\Omega_{s}$ is not (recall our standing assumption $m>0$ ). Therefore, the real-analytic subvariety $\Omega_{e}$ must have codimension one. Also, the set $\Omega_{b} \cap \Omega_{s}$ consists of those points whose images under $\Phi^{\#}$ belong to $\hat{\Omega}_{l}^{\#} \backslash \hat{\Omega}_{l}$ for some $l>0$, and the latter set manifestly has codimension two. Thus, $\Omega_{b} \cap \Omega_{s}$ has codimension two.

To complete the proof of the theorem, we need only show that whenever $P \in \Omega$ is such that there exists $M \in G L(N, C)$ with

$$
M^{-1} L(P) M=\operatorname{diag}\left(\lambda_{1}, \cdots, \lambda_{N}\right)
$$

one must have $\lambda_{j} \neq \lambda_{k}$ for $j \neq k$. We shall now derive a contradiction from the assumption that two $\lambda$ 's are equal. Eventually making a permutation, we may 
assume $\lambda_{1}=\lambda_{2} \equiv \lambda$. Then we obtain as the analog of (2.24)

$$
\left(M^{-1} A M\right)_{j k}\left[1+i\left(\lambda_{j}-\lambda_{k}\right) / \mu g\right]=\left(M^{-1} e\right)_{j}\left(M^{t} e\right)_{k}, \quad \lambda_{1}, \cdots, \lambda_{N} \in S_{\mu g / 2}^{c l}
$$

Viewing this as an equality between $N \times N$ matrices, it follows that the matrix at the lhs has rank one. Since $\left|M^{-1} A M\right|=|A| \neq 0$, this gives rise to a contradiction for $N=2$, so we may assume $N>2$.

Next, we set

$$
r_{k} \equiv 1+i\left(\lambda-\lambda_{k}\right) / \mu g, \quad c_{j} \equiv 1+i\left(\lambda_{j}-\lambda\right) / \mu g, \quad j, k=1, \cdots, N
$$

which entails $r_{1}=r_{2}=c_{1}=c_{2} \neq 0$ and $r_{j}+c_{j} \neq 0$. If all $r_{k}$ would be non-zero, then (5.52) would imply that the first two rows of $M^{-1} A M$ are linearly dependent, contradicting $|A| \neq 0$. Hence, we may as well assume $r_{3}=0$. But then we have $c_{3} \neq 0$ and also

$$
\lambda_{3}=\lambda-i \mu g
$$

Thus, $\lambda$ must belong to the upper boundary and $\lambda_{3}$ to the lower one. Since $\lambda=\lambda_{1}=\lambda_{2}$, this is not possible for $N=3$, so we may assume $N>3$. Likewise, if all $c_{j}$ would be non-zero, then $M^{-1} A M$ would have two linearly dependent columns, so we may assume $c_{4}=0$. But then we have

$$
\lambda_{4}=\lambda+i \mu g
$$

so that $\lambda$ must belong to the lower boundary. This contradiction completes the proof.

The theorem just proved yields a complete elucidation of the state of affairs on $\Omega_{s}$. However, it leaves open some natural questions concerning $\Omega_{e}$. For instance, it does not specify the dimension of $\Omega_{e} \cap \Omega_{b}$, and it does not make clear whether collisions off the real axis can occur. Another open question is: Are there points in $\Omega$ for which $\sigma(L)$ has cardinality one? (We suspect the answer is 'yes' if and only if $\left|N_{+}-N_{-}\right| \leqslant 1$.)

We shall finish this section by exhibiting such phenomena for the special case $N_{+}=N_{-}=2$, taking

$$
x_{1}^{+}=x_{1}^{-}=-x_{2}^{+}=-x_{2}^{-} \equiv x>0, \quad p_{1}^{+}=p_{2}^{+}=-p_{1}^{-}=-p_{2}^{-} \equiv p .
$$

First, choosing 


$$
p=\mu g / 2 \operatorname{ch} \mu x
$$

one finds an $x$-value for which $|\sigma(L)|=1$ :

$$
\operatorname{sh} \mu x=1 \Rightarrow \sigma(L)=\{0\}
$$

Second, with (5.57) in force one obtains, indicating (algebraic) multiplicities,

$$
\begin{array}{ll}
\operatorname{sh} \mu x<1 \Rightarrow \sigma(L)=\{a, a,-a,-a\}, & a \equiv \mu g\left(1-\operatorname{sh}^{2} \mu x\right)^{1 / 2} / 2 \operatorname{sh} \mu x \\
\operatorname{sh} \mu x>1 \Rightarrow \sigma(L)=\{b, b,-b,-b\}, & b \equiv i \mu g\left(\operatorname{sh}^{2} \mu x-1\right)^{1 / 2} / 2 \operatorname{sh} \mu x .
\end{array}
$$

Therefore, multiple collisions and collisions off the real axis cannot be excluded in general. Third, one finds

$$
\operatorname{sh} \mu x=2, p^{2}=9 \mu^{2} g^{2} / 80 \Rightarrow \sigma(L)=\left\{0,0, \frac{1}{2} i \mu g,-\frac{1}{2} i \mu g\right\}
$$

so that $\Omega_{e} \cap \Omega_{b}$ is not empty in general.

\section{B. The Case $\tilde{\Pi}_{\text {rel }}$}

As already mentioned, the cases $\tau \in(0, \pi / 2)$ and $\tau=\pi / 2$ are quite different as regards spectral boundary points. Therefore, we shall first handle the case $\tau \in(0, \pi / 2)$ by following the path laid out in the previous section and then study the $\tau=\pi / 2$ case.

Starting with the special case $N_{+}=N_{-}=1$, one finds that $\Omega_{b}$ is again given by $(5.2)$, whereas

$$
\mathbf{\Omega}_{e}=\left\{\left(x^{+}, x^{-}, p^{+}, p^{-}\right) \in \boldsymbol{R}^{4} \mid \operatorname{th}^{2} \frac{1}{2} \beta\left(p^{+}-p^{-}\right) \operatorname{ch}^{2} \frac{1}{2} \mu\left(x^{+}-x^{-}\right)=\sin ^{2} \tau\right\} .
$$

Thus, $\Omega_{e}$ is a codimension-one submanifold having two connected components, just as (5.1). Again, $L$ is not diagonalizable on $\Omega_{e}$. Changing variables according to (5.3) and using notation introduced below (5.3), one finds that $\Phi_{1}^{\#}$ is given by (5.5) and by

$$
u=-(2 g)^{1 / 2} \sin \frac{1}{2} \beta \delta\left[\frac{1+\delta / \mu g}{\sin ^{2} \tau-\sin ^{2} \frac{1}{2} \beta \delta}\right]^{1 / 2} \operatorname{sh} \frac{1}{2} \mu\left(x^{+}-x^{-}\right)
$$




$$
v=(2 g)^{1 / 2} \cos \frac{1}{2} \beta \delta\left[\frac{1+\delta / \mu g}{\sin ^{2} \tau-\sin ^{2} \frac{1}{2} \beta \delta^{1 / 2}}\right]^{1 / 2} \frac{1}{2} \beta\left(p^{+}-p^{-}\right) \operatorname{ch} \frac{1}{2} \mu\left(x^{+}-x^{-}\right)
$$

where

$$
\begin{gathered}
\delta=-\frac{2}{\beta} \operatorname{Arctg}\left(\left[\sin ^{2} \tau-\operatorname{th}^{2} \frac{1}{2} \beta\left(p^{+}-p^{-}\right) \operatorname{ch}^{2} \frac{1}{2} \mu\left(x^{+}-x^{-}\right)\right] /\right. \\
\left.\left[\operatorname{ch}^{2} \frac{1}{2} \mu\left(x^{+}-x^{-}\right)-\sin ^{2} \tau\right]\right)^{1 / 2} .
\end{gathered}
$$

Also, the inverse transformation $\mathscr{E}_{1}^{\#}$ reads

$$
\begin{aligned}
& x^{ \pm}=x \pm \frac{1}{\mu} \operatorname{Arth}(u r) \\
& p^{ \pm}=\frac{1}{2} p \pm \frac{1}{\beta} \operatorname{Arth}\left(v r \operatorname{tg}\left[\tau-\frac{1}{4} \beta \mu\left(u^{2}+v^{2}\right)\right]\right)
\end{aligned}
$$

where

$$
r \equiv\left[\frac{\sin ^{2} \tau-\sin ^{2}\left[\tau-\frac{1}{4} \beta \mu\left(u^{2}+v^{2}\right)\right]}{u^{2} \sin ^{2} \tau+v^{2} \sin ^{2}\left[\tau-\frac{1}{4} \beta \mu\left(u^{2}+v^{2}\right)\right]}\right]^{1 / 2}
$$

As expected, these formulas reduce to (5.6)-(5.10) when $\beta \rightarrow 0$. (Recall $\tau \equiv \beta \mu g / 2$.) Once again, $\Phi_{1}^{\#}$ and $\mathscr{E}_{1}^{\#}$ are symplectomorphisms w.r.t. (5.11), and (5.12) holds true.

We continue with the general case. Replacing (5.13) by its $\tilde{\Pi}_{r e l}$ generalization

$$
S_{k}(\hat{q}, \hat{\theta})=\sum_{|I|=k} \exp \left(\mu \sum_{i \in I} \hat{q}_{i}\right) \prod_{\substack{i \in I \\ j \notin I}}\left[\operatorname{sh}^{2} \frac{1}{2} \beta\left(\hat{\theta}_{i}-\hat{\theta}_{j}\right)+\sin ^{2} \tau\right]^{1 / 2} / \operatorname{sh} \frac{1}{2} \beta\left(\hat{\theta}_{\min (i, j)}-\hat{\theta}_{\max (i, j)}\right)
$$

(cf. (3.57)-(3.60) and (B4)) we shall first show $\Omega_{b}$ is non-empty. To this end we follow the reasoning after (5.13), replacing (5.14) by 


$$
\frac{1}{2} \operatorname{Tr}\left[\mathscr{A}(\tilde{\hat{P}})+\mathscr{A}(\tilde{\hat{P}})^{-1}\right]=\frac{1}{2} \operatorname{Tr}\left[L(P)+L(P)^{-1}\right]=P_{0}(P)
$$

where $P_{0}$ is the Hamiltonian

$$
P_{0} \equiv \sum_{i=1}^{N_{+}} \operatorname{ch}\left(\beta p_{i}^{+}\right) V_{i}^{+}\left(x^{+}, x^{-}\right)+\sum_{j=1}^{N_{-}} \operatorname{ch}\left(\beta p_{j}^{-}\right) V_{j}^{-}\left(x^{+}, x^{-}\right)
$$

cf. (1.3), (1.4). (The second equality in (5.69) follows by using (B4).) From this the desired a priori estimates readily follow. (Note $H=\beta^{-1} \operatorname{Tr} L$ cannot be used, since $e^{x}$ is not bounded away from zero.)

Next, we may and will copy the definitions and relations (5.15)-(5.21), which prepares us for the following theorem.

Theorem 5.2. For $\tau \in(0, \pi / 2)$ the assertions of Theorem 5.1 hold true.

Proof. The reasoning in the proof of Theorem 5.1 applies nearly verbatim, with Theorem 4.2, Lemma 3.4, (5.69) and Section 2C playing the role of Theorem 4.1, Lemma 3.1, (5.14) and Section 2B, resp. Therefore, we only list the changes, to wit: $(5.29),(5.39) \rightarrow$

$$
\begin{aligned}
&\left(\mathscr{L}_{r}\right)_{j k}= \exp \left(2 \mu x_{n}+\beta \operatorname{Re} a_{j}\right) \sin \tau \sin \left(\tau+\beta \operatorname{Im} a_{j}\right)\left(\sin \beta \operatorname{Im} a_{j}\right)^{-2} \\
& \cdot \prod_{i \neq j, k} \frac{\left[\operatorname{sh}^{2} \frac{1}{2} \beta\left(a_{j}-a_{i}\right)+\sin ^{2} \tau\right]^{1 / 2}}{\operatorname{sh} \frac{1}{2} \beta\left(a_{j}-a_{i}\right)} \cdot \frac{\left[\operatorname{sh}^{2} \frac{1}{2} \beta\left(a_{k}-a_{i}\right)+\sin ^{2} \tau\right]^{1 / 2}}{\operatorname{sh} \frac{1}{2} \beta\left(a_{k}-a_{i}\right)} \\
& \mathscr{P}_{r} L \mathscr{P}_{r}^{-1}=\operatorname{diag}\left(\exp \left(\beta a_{1}\right), \cdots, \exp \left(\beta a_{N}\right)\right),
\end{aligned}
$$

resp.; $v_{k} \rightarrow \exp \left(-\beta a_{k}\right) v_{k}$ at the lhs of (5.45); (5.46), (5.48), (5.49) $\rightarrow$

$$
\begin{gathered}
v_{j}=(-)^{j-1} \exp \left(\mu x_{n}+\beta a_{j}\right)\left(u_{n}-i v_{n}\right)(\beta \mu)^{1 / 2}\left(2 \sin \beta \operatorname{Im} a_{j}\right)^{-1} \\
{\left[\frac{\sin \left(\tau-\beta \operatorname{Im} a_{j}\right) \sin \left(\tau+\beta \operatorname{Im} a_{j}\right)}{\tau-\beta \operatorname{Im} a_{j}}\right]^{1 / 2} \prod_{i \neq j, j+l} \frac{\left[\operatorname{sh}^{2} \frac{1}{2} \beta\left(a_{j}-a_{i}\right)+\sin ^{2} \tau\right]^{1 / 2}}{\operatorname{sh} \frac{1}{2} \beta\left(a_{j}-a_{i}\right)}} \\
\hat{e}_{j} \tilde{e}_{k} / \hat{A}_{j k}=\left(u_{n}^{2}+v_{n}^{2}\right) \frac{\beta \mu}{4 \sin \tau} \exp \left(\beta \operatorname{Re} a_{j}\right) \frac{\sin \left(\tau-\beta \operatorname{Im} a_{j}\right)}{\tau-\beta \operatorname{Im} a_{j}}
\end{gathered}
$$




$$
\hat{e}_{j} \tilde{e}_{k} / \hat{A}_{j k}=\exp \left(\beta \operatorname{Re} a_{j}\right)\left[\cos \left(\beta \operatorname{Im} a_{j}\right)-\cot \tau \sin \left(\beta \operatorname{Im} a_{j}\right)\right]
$$

resp.; (5.52)-(5.55) $\rightarrow$

$$
\begin{aligned}
& \left(M^{-1} A M\right)_{j k}\left[\frac{1}{2}\left(\lambda_{j}+\lambda_{k}\right)+\frac{i}{2} \cot \tau\left(\lambda_{j}-\lambda_{k}\right)\right]=\left(M^{-1} e\right)_{j}\left(M^{t} e\right)_{k}, \\
& \lambda_{1}, \cdots, \lambda_{N} \in \exp \left(S_{\tau}^{c l}\right) \\
& r_{k} \equiv \frac{1}{2}\left(\lambda+\lambda_{k}\right)+\frac{i}{2} \cot \tau\left(\lambda-\lambda_{k}\right), \quad c_{j} \equiv \frac{1}{2}\left(\lambda_{j}+\lambda\right)+\frac{i}{2} \cot \tau\left(\lambda_{j}-\lambda\right) \\
& \lambda_{3}=\lambda \exp (-2 i \tau) \\
& \lambda_{4}=\lambda \exp (2 i \tau)
\end{aligned}
$$

resp. (Note $r_{j}+c_{j} \neq 0$ since $\operatorname{Re} \lambda_{i}>0, i=1, \cdots, N$.)

The first paragraph after Theorem 5.1 applies again. Moreover, the situation concerning collisions for the special case $N_{+}=N_{-}=2$ is the same as before. Indeed, consider again points in $\Omega$ of the form (5.56), Choosing first

$$
\operatorname{ch} \beta p=\left[1-\sin ^{2} \tau / \operatorname{ch}^{2} \mu x\right]^{-1 / 2}
$$

one finds as the generalization of (5.58)-(5.60)

$$
\sigma(L)=\left\{e^{\alpha}, e^{\alpha}, e^{-\alpha}, e^{-\alpha}\right\},
$$

where $\alpha$ is defined by

$$
\operatorname{ch} \alpha=\cos \tau\left[1+\sin ^{2} \tau / \operatorname{sh}^{2} \mu x\right]^{1 / 2} .
$$

(In particular, $\sigma(L)=\{1\}$ when $\operatorname{sh} \mu x=\cos \tau$.) Also, one obtains

$$
\sigma(L)=\{1,1, \exp (i \tau), \exp (-i \tau)\}
$$

(and, therefore, a point in $\Omega_{e} \cap \Omega_{b}$ ), when one chooses

$$
\begin{aligned}
& \operatorname{sh} \mu x=\left[2 \cos \tau+2 \cos ^{2} \tau\right]^{1 / 2} \\
& \operatorname{ch} \beta p=\frac{1}{\cos ^{2} \tau}\left[\frac{1+2 \cos \tau+2 \cos ^{2} \tau}{4+6 \cos \tau}\right]^{1 / 2}\left[(1+\cos \tau)^{1 / 2}-\sin \tau(1-\cos \tau)^{1 / 2}\right] .
\end{aligned}
$$


We proceed by studying the case $\tau=\pi / 2$, taking again $N_{+}=N_{-}=1$ for a first orientation. From (2.70) one then infers

$$
x^{+}-x^{-} \rightarrow 0 \pm \Rightarrow L \rightarrow \pm i \exp \left(\frac{1}{2} \beta\left[p^{+}+p^{-}\right]\right)\left[\begin{array}{ll}
0 & 1 \\
1 & 0
\end{array}\right] \text {. }
$$

Thus, $L$ has a jump discontinuity on $\Omega \backslash \Omega^{b}$, but $\sigma(L)$ is continuous. Consequently, one may include the hypersurface $x^{+}=x^{-}$as regards spectral properties. Doing so, one finds

$$
\mathbf{\Omega}_{b}=\mathbf{\Omega} \backslash \mathbf{\Omega}^{b}=\left\{\left(x^{+}, x^{-}, p^{+}, p^{-}\right) \in \boldsymbol{R}^{4} \mid x^{+}=x^{-}\right\}
$$

whereas $\Omega_{e}$ is given by (5.62) with $\tau=\pi / 2$. Thus, $\Omega_{e}$ now consists of four disjoint hypersurfaces. Moreover, after the change of variables (5.3) one finds that $\Phi_{1}$ and $\mathscr{E}_{1}$ are given by (5.5) and (5.63)-(5.67) with $\tau=\pi / 2$, but now these realanalytic symplectomorphisms do not have sensible extensions. Indeed, when $x^{+} \rightarrow x^{-}$one gets $u, v \rightarrow 0$ for any $p^{+}, p^{-}$; when $u, v \rightarrow 0$ one gets $x^{+} \rightarrow x^{-}$, whereas $p^{+}, p^{-}$have direction-dependent limits.

Next, we turn to the general case. The formulas (5.68)-(5.70) now do not lead to useful conclusions, since the potentials in $P_{0}$ are not bounded away from zero for $\tau=\pi / 2$, cf. (1.3), (1.4). However, it is not hard to see directly that the Lax matrix has no imaginary eigenvalues on $\Omega^{b}$. Indeed, let $P \in \Omega^{b}$ and assume $L(P) \varphi=\lambda \varphi, \varphi \neq 0, \operatorname{Re} \lambda=0$. Then it follows that $\left(\varphi,\left(L+L^{*}\right) \varphi\right)=0$. But from (2.70) one obtains

$$
\frac{1}{2}\left(L+L^{*}\right)=\left[\begin{array}{ll}
L_{++} & 0 \\
0 & L_{--}
\end{array}\right]=D_{+-}\left[\begin{array}{cc}
L\left(N_{+}\right) & 0 \\
0 & L\left(N_{-}\right)
\end{array}\right] D_{+-}
$$

where

$$
D_{+-} \equiv \operatorname{diag}\left(\prod_{1 \leqslant j \leqslant N_{-}}\left|\operatorname{th} \frac{1}{2} \mu\left(x_{1}^{+}-x_{j}^{-}\right)\right|^{1 / 2}, \cdots, \prod_{1 \leqslant i \leqslant N_{+}}\left|\operatorname{th} \frac{1}{2} \mu\left(x_{N}^{-}-x_{i}^{+}\right)\right|^{1 / 2}\right)
$$

and where $L\left(N_{+}\right) / L\left(N_{-}\right)$are pure soliton/antisoliton Lax matrices. Since all of these matrices are positive on $\Omega^{b}$, one has $L+L^{*}>0$, so that $\varphi=0$. This is a contradiction, so that no spectral boundary points can arise on $\Omega^{b}$.

Just as for the above special case, $\sigma(L)$ has a continuous extension to $\Omega$, whereas $L$ has jump discontinuities on $\Omega \backslash \Omega^{b}$. For example, from (2.70) and 
(1.3), (1.4) one reads off

$$
x_{i}^{+}-x_{j}^{-} \rightarrow 0 \pm \Rightarrow\left\{\begin{array}{l}
L_{i, N_{+}+j}, L_{N_{+}+j, i} \rightarrow \pm i \exp \left(\frac{1}{2} \beta\left[p_{i}^{+}+p_{j}^{-}\right]\right) \\
L_{i k}, L_{k i}, L_{l, N_{+}+j}, L_{N_{+}+j, l} \rightarrow 0, \quad k \neq N_{+}+j, \quad l \neq i
\end{array}\right.
$$

whereas the remaining matrix elements lose their dependence on $x_{i}^{+}$and $x_{j}^{-}$. Thus, any point in $\Omega \backslash \Omega^{b}$ yields imaginary eigenvalues and the corresponding dead breathers are forgotten altogether, in contrast to the situation for $\tau<\pi / 2$.

We now summarize and extend these findings.

Theorem 5.3. Let $\tau=\pi / 2$. Then one has

$$
\Omega_{b}=\Omega \backslash \Omega^{b}, \quad \Omega_{r}^{b}=\Omega_{s}^{b} .
$$

The set $\Omega_{e}^{b} \equiv \Omega^{b} \backslash \Omega_{s}^{b}$ has codimension one and $L$ is not diagonalizable on $\Omega_{e}^{b}$.

Proof. We have shown above that no point in $\Omega^{b}$ yields imaginary eigenvalues, whereas any point in $\Omega \backslash \Omega^{b}$ does, cf. (5.89). Hence (5.90) follows. Next, we recall that we have already proved that $\Omega^{b} \backslash \Omega_{r}^{b}$ has codimension one, cf. the last paragraph of Section 4B. Since $\Omega_{e}^{b}=\Omega^{b} \backslash \Omega_{r}^{b}$, it remains to prove non-diagonalizability of $L$ on $\Omega_{e}^{b}$. But this follows as before; in fact, already (5.78) yields a contradiction, since $\operatorname{Re} \lambda_{j}>0$.

The points (5.56) do not belong to $\Omega^{b}$ and hence cannot be used to obtain illuminating examples for $\tau=\pi / 2$. However, just as in the previous cases it is easy to see that for $N_{+}=2, N_{-}=1$ there exist points in $\Omega_{e}^{b}$ yielding $|\sigma(L)|=1$.

\section{Dynamics and Scattering}

\section{A. The Case $\tilde{I I}_{n r}$}

Thus far, we have been engaged in the construction and study of the action-angle transform $\Phi$ and its harmonic oscillator extension $\Phi^{\sharp}$. In this enterprise the Hamiltonian (1.1) has been invoked only once, namely to yield bounds relevant for studying the boundary set $\Omega_{b}$, cf. (5.14). However, it should be stressed at this point that we proved the crucial canonicity property of $\Phi^{(\#)}$ by reduction to the case $m=0$ studied in I, and our canonicity proof 
for this special case does make essential use of prior information concerning the $H$ flow and its temporal asymptotics, cf. I.

We proceed by obtaining detailed information on the $m>0 \mathrm{H}$ flow and its asymptotics. In fact, we shall study along with $H=\frac{1}{2} \operatorname{Tr} L^{2}$ the class of Hamiltonians of the form

$$
H_{h} \equiv \operatorname{Tr} h(L), \quad h \in \mathscr{C}
$$

where $\mathscr{C}$ is the class of all entire functions $h$ of the form $h(z)=\sum_{n=0}^{\infty} a_{n} z^{n}$, $a_{n} \in \boldsymbol{R}$. Since the Hamiltonians $\operatorname{Tr} L^{n}$ are real-valued real-analytic functions on $\Omega$ (recall $L$ is $\mathscr{J}$-s.a. and real-analytic), the same is true for any $H_{h}$.

We begin by noting that the $H$ flow is complete on all of $\Omega$. Indeed, conservation of $H$ yields both a non-zero lower bound on the distances $\left|x_{i}^{\delta}-x_{j}^{\delta}\right|$, $i \neq j, \delta=+,-$ (so that any integral curve stays away from $\partial \Omega$ ) and an upper bound on $\left|p^{+}\right|$and $\left|p^{-}\right|$(so that the particles cannot escape to infinity in finite time).

To handle, more generally, the $H_{h}$ flow, we shall make extensive use of the relations

$$
L(P) \sim \tilde{L}(\hat{P}), \quad A(P) \sim \tilde{A}(\hat{P}), \quad P \in \Omega_{l}, \quad \hat{P} \equiv \Phi_{l}(P) \in \hat{\Omega}_{l} .
$$

(Here and from now on, $\sim$ denotes similarity. Also the matrices $\tilde{A}$ and $\tilde{L}$, originally defined by (2.47), (2.48), are here viewed as functions on $\hat{\Omega}_{l}$ via the coordinate change $(4.1)-(4.3)$.) We begin by introducing the Hamiltonians

$$
\hat{H}_{h, l} \equiv H_{h} \circ \mathscr{E}_{l}, \quad l=0, \cdots, m, \quad h \in \mathscr{C}
$$

on $\hat{\Omega}_{l}$. In view of (6.1) and (6.2) we have

$$
\hat{H}_{h, l}(\hat{P})=\sum_{i=1}^{k_{+}} h\left(p_{i}^{s}\right)+\sum_{j=1}^{k_{-}} h\left(p_{j}^{\bar{s}}\right)+2 \sum_{n=1}^{l} \operatorname{Reh}\left(\frac{1}{2}\left(p_{n}-i \delta_{n}\right)\right)
$$

cf. also Section 4A. It is evident from this that all of these Hamiltonians commute, and since $\mathscr{E}_{l}$ is symplectic, the Hamiltonians $H_{h}$ commute on $\Omega_{l}$, and hence on the open dense subset $\Omega_{r}$ of $\Omega$. On account of their real-analyticity, they then commute on all of $\Omega$. In particular, $H$ is conserved under the $H_{h}$ flow, so that any $H_{h}$ integral curve stays away from $\partial \Omega$ and has bounded $\left|p^{+}\right|$ and $\left|p^{-}\right|$. Therefore, completeness of the integral curve $\exp \left(t H_{h}\right)(P)$ through 
$P \in \Omega$ will follow if we can prove that the position part of the curve does not diverge in finite time.

Before deriving this from an explicit description of the position part, it should be noticed that completeness of $\exp \left(t H_{h}\right)$ on $\Omega_{r}$ easily follows from (6.3) and (6.4). Indeed, due to (6.3) one has

$$
\exp \left(t H_{h}\right) \mathscr{E}_{l}=\mathscr{E}_{l} \exp \left(t \hat{H}_{h, l}\right)
$$

and it is obvious from (6.4) that the $\hat{H}_{h, l}$ flow reads

$$
\begin{gathered}
\exp \left(t \hat{H}_{h . l}\right)\left(x^{s}, x^{\bar{s}}, p^{s}, p^{\bar{s}} ; x, \gamma, p, \delta\right)=\left(x_{1}^{s}+t h^{\prime}\left(p_{1}^{s}\right), \cdots, x_{k_{-}}^{\bar{s}}+t h^{\prime}\left(p_{k_{-}}^{\bar{s}}\right), p^{s}, p^{\bar{s}} ;\right. \\
\left.x_{1}+t \operatorname{Re} h^{\prime}\left(\frac{1}{2}\left(p_{1}-i \delta_{1}\right)\right), \cdots, \gamma_{l}+t \operatorname{Im} h^{\prime}\left(\frac{1}{2}\left(p_{l}-i \delta_{l}\right)\right), p, \delta\right)
\end{gathered}
$$

and so is complete on $\hat{\Omega}_{l}$. (Using the results of Section $5 A$ it is easy to extend the previous argument to $\Omega_{s}$ ) However, completeness of a Hamiltonian flow is not implied by completeness on an open dense set, so we need an extra argument to handle the general case. Among other things, such an argument is provided by the following theorem, which involves the matrix

$$
A_{h}(t, P) \equiv A(P) \exp \left(t \mu h^{\prime}(L(P))\right)
$$

Theorem 6.1. Let $h \in \mathscr{C}, t \in \mathbb{R}$ and $P \in \Omega$. Then the matrix $A_{h}(t, P)$ has simple and real spectrum and its eigenvalues $\alpha_{1}(t), \cdots, \alpha_{N}(t)$ can be ordered such that

$$
\alpha_{1}(t)>\cdots>\alpha_{N_{+}}(t)>0, \quad \alpha_{N_{+}+1}(t)<\cdots<\alpha_{N}(t)<0 .
$$

Moreover, the integral curve $\exp \left(t H_{h}\right)(P)$ is complete and its position part is given by

$$
\begin{aligned}
& x_{i}^{+}(t)=\mu^{-1} \ln \left(\alpha_{i}(t)\right), \quad i=1, \cdots, N_{+} \\
& x_{j}^{-}(t)=\mu^{-1} \ln \left(-\alpha_{N_{+}+j}(t)\right), \quad j=1, \cdots, N_{-} .
\end{aligned}
$$

Proof. We have already shown that the $H_{h}$ flow is complete on $\Omega_{l}$, cf. (6.5), (6.6). Taking first $P \in \Omega_{l}$, we claim that

$$
A\left(\exp \left(t H_{h}\right)(P)\right) \sim A_{h}(t, P) .
$$

To prove this, we note that on the one hand 


$$
A\left(\exp \left(t H_{h}\right)(P)\right)=A\left(\mathscr{E}_{l} \exp \left(t \hat{H}_{h, l}\right) \Phi_{l}(P)\right) \sim \tilde{A}\left(\exp \left(t \hat{H}_{h, l}\right)(\hat{P})\right)
$$

where we used (6.5) in the first step and (6.2) in the second one. On the other hand, we infer from (6.6) and the explicit form of $\tilde{A}$ and $\tilde{L}$ (cf. (2.47), (2.48) and (4.1)-(4.3)) that

$$
\tilde{A}\left(\exp \left(t \hat{H}_{h, l}\right)(\hat{P})\right)=\tilde{A}(\hat{P}) \exp \left(t \mu h^{\prime}(\tilde{L}(\hat{P}))\right) \sim A_{h}(t, P) .
$$

(To verify the second step one need only recall that the similarities in (6.2) are effected by the same matrix.) Thus, (6.10) follows for $P \in \Omega_{l}$, as claimed.

From (6.10) and the definition (2.6) of $A$ one now sees that the theorem holds true for any $P \in \Omega_{l}$ and hence for any $P \in \Omega_{r}$. To handle the general case, we observe that by virtue of standard ODE lore we can find a time interval $\left[-\epsilon_{0}, \epsilon_{0}\right]$ and a closed ball $B_{0} \subset \Omega$ around any $P_{0} \in \Omega$ such that the map $\left[-\epsilon_{0}, \epsilon_{0}\right] \times B_{0} \rightarrow \Omega,(t, P) \mapsto \exp \left(t H_{h}\right)(P)$ is well defined and real-analytic. On the other hand, the matrix $A_{h}(t, P)$ has simple spectrum on $\{0\} \times B_{0}$, so one can find $\epsilon_{1} \in\left(0, \epsilon_{0}\right]$ such that it has simple spectrum and real-analytic eigenvalues on $\left[-\epsilon_{1}, \epsilon_{1}\right] \times B_{0}$. Furthermore, since $\Omega_{r} \cap B_{0}$ is dense in $B_{0}$, the position part of $\exp \left(t H_{h}\right)\left(P_{0}\right)$ must be related to the eigenvalues of $A_{h}\left(t, P_{0}\right)$ via (6.9) for any $t \in\left[-\epsilon_{1}, \epsilon_{1}\right]$.

A moment's thought now shows that a contradiction arises when one assumes that the maximal $\epsilon\left(P_{0}\right)$ for which the relation holds is finite. Indeed, we have already seen that the integral curve $\exp \left(t H_{h}\right)\left(P_{0}\right)$ can only fail to be complete due to divergence in finite time of its position part, whereas the spectrum of $A_{h}\left(t, P_{0}\right)$ clearly stays away from 0 and $\pm \infty$ for finite times.

The theorem just proved entails that the $|t| \rightarrow \infty$ asymptotics of the position part of the $H_{h}$ integral curve through $P$ reduces to the spectral asymptotics of the matrix $A_{h}(t, P)$. To study this, we first choose $P \in \Omega_{l}$. Recalling the diagonal similarity transformation (2.54), we deduce from (6.12)

$$
A_{h}(t, P) \sim M_{l} \hat{\grave{J}} \exp \left(t \mu h^{\prime}(\tilde{L}(\hat{P}))\right), \quad P \in \Omega_{l}
$$

where we have introduced the positive matrix

$$
M_{l} \equiv A_{s} \hat{\mathscr{F}}
$$

Combining (2.50) -(2.55) with (4.1)-(4.3), we may and will view $M_{l}$ as a function on $\hat{\Omega}_{l}$, explicitly given by 


$$
M_{l}=S C(0, \mu,-g ; a, \bar{a}) S^{*}
$$

where

$$
\begin{aligned}
& S=\operatorname{diag}\left(s_{1}, \cdots, s_{N}\right) \equiv \operatorname{diag}\left(\exp \left(\frac{1}{2} \mu x_{1}^{s}\right)\left|\mathscr{V}_{1}(a)\right|^{1 / 2}, \cdots\right. \\
& \exp \left(\frac{1}{2} \mu x_{k_{+}}^{s}\right)\left|\mathscr{V}_{k_{+}}(a)\right|^{1 / 2},(-)^{k_{+}} \exp \left(\mu\left[x_{1}+i \gamma_{1}\right]\right) \mathscr{V}_{k_{+}+1}(a), \cdots \\
& (-)^{k_{+}+l-1} \exp \left(\mu\left[x_{l}+i \gamma_{l}\right]\right) \mathscr{V}_{k_{+}+l}(a), 1, \cdots, 1, \exp \left(\frac{1}{2} \mu x_{1}^{\bar{s}}\right)\left|\mathscr{V}_{N-k_{-}+1}(a)\right|^{1 / 2}, \cdots \\
& \left.\exp \left(\frac{1}{2} \mu x_{k_{-}}^{\bar{s}}\right)\left|\mathscr{V}_{N}(a)\right|^{1 / 2}\right) \\
& a \equiv\left(p_{1}^{s}, \cdots, p_{k_{+}}^{s}, \frac{1}{2}\left(p_{1}-i \delta_{1}\right), \cdots, \frac{1}{2}\left(p_{l}+i \delta_{l}\right), p_{1}^{\bar{s}}, \cdots, p_{k_{-}}^{\bar{s}}\right) .
\end{aligned}
$$

From this one readily infers that $M_{l}$ has a continuous and positive extension to $\hat{\Omega}_{l}^{\#}$, cf. Section 5A. Indeed, as $\delta_{n} \downarrow-\mu g$, the matrix element $C_{k_{+}+n, k_{+}+n}$ diverges, but $\left(M_{l}\right)_{k_{+}+n, k_{+}+n}$ does not, since $\mathscr{V}_{k_{+}+n} \rightarrow 0$. Explicitly, one obtains

$$
\delta_{n} \downarrow-\mu g \Rightarrow\left(M_{l}\right)_{i j} \rightarrow\left\{\begin{array}{ccc}
0, & i=k_{+}+n, & j \neq k_{+}+n \\
0, & i \neq k_{+}+n, & j=k_{+}+n \\
c_{n}, & i=k_{+}+n, & j=k_{+}+n
\end{array}\right.
$$

where

$$
c_{n} \equiv 2 \exp \left(2 \mu x_{n}\right) \prod_{j \neq k_{+}+n, k_{+}+l+n}\left|1+\frac{\mu^{2} g^{2}}{\left(a_{k_{+}+n}-a_{j}\right)^{2}}\right| .
$$

We proceed by introducing real numbers $\xi_{j}, \eta_{j}$ via

$$
\begin{aligned}
\left(\mu h^{\prime}\left(a_{1}\right), \cdots, \mu h^{\prime}\left(a_{N}\right)\right) \equiv & \left(\xi_{1}, \cdots, \xi_{k_{+}}, \xi_{k_{+}+1}+i \eta_{1}, \cdots, \xi_{k_{+}+l}+i \eta_{l},\right. \\
& \left.\xi_{k_{+}+1}-i \eta_{1}, \cdots, \xi_{k_{+}+l}-i \eta_{l}, \xi_{N-k_{-}+1}, \cdots, \xi_{N}\right)
\end{aligned}
$$

and then set

$$
\left\{\xi_{1}, \cdots, \xi_{N}\right\} \equiv\left\{d_{1}, \cdots, d_{K}\right\}, \quad d_{K}<\cdots<d_{1}, \quad 1 \leqslant K \leqslant N-l
$$

Next, we define a permutation $\sigma$, as follows: first, we permute the rhs of $(6.20)$ into 


$$
\left(\xi_{1}, \cdots, \xi_{k_{+}}, \xi_{k_{+}+1}+i \eta_{1}, \xi_{k_{+}+1}-i \eta_{1}, \cdots, \xi_{k_{+}+l}+i \eta_{l}, \xi_{k_{+}+l}-i \eta_{l}, \xi_{N-k_{-}+1}, \cdots, \xi_{N}\right)
$$

(so that $\hat{\mathscr{J}}$ turns into $\operatorname{diag}\left(\mathbf{1}_{k_{+}}, \sigma_{1}, \cdots, \sigma_{1},-\mathbf{1}_{k_{-}}\right), \sigma_{1} \equiv\left[\begin{array}{ll}0 & 1 \\ 1 & 0\end{array}\right]$ occurring $l$ times), and then we reorder (6.22) so that the real parts are decreasing from left to right, retaining the ordering of (6.22) in each cluster.

We now transform the rhs of (6.13) with the permutation matrix $\Pi_{\sigma}$ representing the permutation $\sigma$ just defined. This yields

$$
A_{h}(t, P) \sim E(t), \quad P \in \Omega_{l}
$$

where $E(t)$ is given by (C4) with

$$
\begin{gathered}
M=\Pi_{\sigma} M_{l} \Pi_{\sigma}^{-1} \\
\mathscr{J}_{\rho}(t)=\operatorname{diag}\left(\mathbf{1}_{n(s)},\left[\begin{array}{lc}
0 & \exp \left(-i t \eta_{n_{1}}\right) \\
\exp \left(i t \eta_{n_{1}}\right) & 0
\end{array}\right], \cdots,\left[\begin{array}{lc}
0 & \exp \left(-i t \eta_{n_{n(b)}}\right) \\
\exp \left(i t \eta_{n_{n(b)}}\right) & 0
\end{array}\right]\right. \\
\left.-\mathbf{1}_{n(\bar{s})}\right)
\end{gathered}
$$

and with $d_{1}, \cdots, d_{K}$ defined via (6.20), (6.21); thus one has

$$
\left|I_{\rho}\right|=n(s)+2 n(b)+n(\bar{s})
$$

The point of these transformations is, that the assumptions (C3), (C5), (C6) are now satisfied. Therefore, we may read off from Theorem $\mathrm{C} 1$ the gross features of the temporal asymptotics of the positions $x_{N_{+}}^{+}(t)<\cdots<x_{1}^{+}(t)$, $x_{N_{-}}^{-}(t)<\cdots<x_{1}^{-}(t)$ : For $|t| \rightarrow \infty$ there are $K$ separated clusters moving with velocities $d_{K}<\cdots<d_{1}$ and the number of solitons, antisolitons and breathers in each cluster stays the same. (However, rearrangements must occur in general, so as to preserve the two orderings involved.)

To study the asymptotics in more detail, we should determine the cluster matrices $(\mathrm{C} 10)$ for the case at hand. Since $\mathscr{J}_{\rho}(t)$ is given by $(6.25)$, we need only calculate the matrices $m_{\rho}^{\delta}$ defined by (C9). We begin by doing so when $M$ is of the form

$$
M_{j k}=f_{j} C(0, \mu,-g ; p, \bar{p})_{j k} \bar{f}_{k}, \quad f_{j} \in C^{*}, \quad j, k=1, \cdots, N
$$

(Here, $p \in C^{N}$ is chosen such that Lemma $\mathrm{B} 1$ applies.) To this end we invoke 
Lemma B2: it enables us to calculate the inverses of the three Cauchy matrices that are involved. Explicitly, this yields

$$
\left(m_{\rho}^{\delta}\right)_{j-n_{\rho-1}, k-n_{\rho-1}}=g_{j}^{\delta} M_{j k} \overline{g_{k}^{\delta}}, \quad \delta=+,-, \quad j, k \in I_{\rho}
$$

where

$$
g_{j}^{\delta} \equiv \prod_{m \in I_{\rho}^{\delta}} \frac{p_{j}-p_{m}}{p_{j}-\bar{p}_{m}-i \mu g}, \quad j \in I_{\rho}
$$

(Recall (C1) and (C8).)

In the case at hand $M$ is of the form (6.27) with

$$
f_{j}=s_{\sigma^{-1}(j)}, \quad p_{j}=a_{\sigma^{-1}(j)}
$$

provided $P \in \Omega_{l}$. However, (6.28) holds true for $P \in \Omega_{l}^{\#}$, too, since all eigenvalues of $M$ stay away from 0 on $\hat{\Omega}_{l}^{\#}$, cf, (6.24), (6.14).

We are now in the position to determine the asymptotics of the center of mass position of cluster $\rho$ by combining Theorem $\mathrm{C} 1$ with the explicit formulas just derived. To determine the asymptotics of the individual positions is not feasible in general; we shall only handle the three cases in which $n(s)+n(b)+n(\vec{s})=1$. Since $h(z)$ is assumed to be entire, the subset of $\Omega_{l}^{\#}$ for which all clusters satisfy this restriction is an open dense full measure subset whenever $h$ depends nonlinearly on $z$. In particular, for the Hamiltonian $H$ (for which $h(z)=\frac{1}{2} z^{2}$, cf. (2.23)) this subset is given by

$$
\Omega_{l \neq}^{\#} \equiv\left\{P \in \Omega_{l}^{\#} \mid p_{1}^{s}, \cdots, p_{k_{+}}^{s}, p_{1}^{\bar{s}}, \cdots, p_{k_{-}}^{\bar{s}}, \frac{1}{2} p_{1}, \cdots, \frac{1}{2} p_{l} \text { distinct }\right\}
$$

The scattering will be detailed in terms of the center shift and phase shift functions

$$
\begin{aligned}
& \delta_{c}: S_{\mu g}^{c l} \backslash\{0, \pm i \mu g\} \rightarrow \boldsymbol{R}, \quad z \mapsto \mu^{-1} \ln \left|1+\mu^{2} g^{2} / z^{2}\right| \\
& \delta_{p}: S_{\mu g} \backslash\{0\} \rightarrow 2 \mu^{-1}(-\pi, \pi], \quad z \mapsto 2 \mu^{-1} \arg \left(\left[z^{2}+\mu^{2} g^{2}\right]^{1 / 2} / z\right) .
\end{aligned}
$$

Here, $S_{\mu g}$ is defined by (2.27) and the square root sign is defined by requiring

$$
\operatorname{Re}\left[z^{2}+\mu^{2} g^{2}\right]^{1 / 2}>0, \quad z \in S_{\mu g}
$$

Theorem 6.2. Let $P \in \Omega_{l}^{\#}$ and let $x^{+}(t), x^{-}(t)$ denote the position part of 
the integral curve $\exp \left(t H_{h}\right)(P)$. Let $\sigma$ be the permutation defined in the paragraph containing (6.22). Let $\rho \in\{1, \cdots, K\}$ and let $d_{\rho}$ be defined via (6.20), (6.21). Finally, let $\mathscr{I}_{\rho}(t),\left|I_{\rho}\right|$ and $r_{\rho}$ be given by (6.25), (6.26) and $(C 12)$, resp. Then there exist uniquely determined indices $i_{1}, \cdots, i_{n(s)} \in\left\{1, \cdots, k_{+}\right\}, j_{1}, \cdots, j_{n(s)} \in\left\{1, \cdots, k_{-}\right\}, n_{1}, \cdots$, $n_{n(b)} \in\{1, \cdots, l\}, i_{\delta, 1}, \cdots, i_{\delta, n(s)+n(b)} \in\left\{1, \cdots, N_{+}\right\}, j_{\delta, 1}, \cdots, j_{\delta, n(\tilde{s})+n(b)} \in\left\{1, \cdots, N_{-}\right\}$such that

$$
\begin{gathered}
\frac{1}{\left|I_{\rho}\right|}\left[\sum_{k=1}^{n(s)+n(b)} x_{i_{\delta, k}}^{+}(t)+\sum_{k=1}^{n(s)+n(b)} x_{j_{\delta, k}}^{-}(t)\right]=\frac{1}{\left|I_{\rho}\right|}\left[\sum_{k=1}^{n(s)} x_{i_{k}}^{s}+\sum_{k=1}^{n(s)} x_{j_{k}}^{\bar{s}}+2 \sum_{k=1}^{n(b)} x_{n_{k}}\right] \\
-\frac{\delta}{2} \Delta_{\rho}+t d_{\rho} / \mu+O\left(\exp \left(-|t| r_{\rho}\right)\right), \quad t \rightarrow \delta \infty
\end{gathered}
$$

where $\delta=+,-$ and where

$$
\Delta_{\rho} \equiv \frac{1}{\left|I_{\rho}\right|} \sum_{j \in I_{\rho}}\left[\sum_{k \in I_{\rho}^{+}}-\sum_{k \in I_{\bar{\rho}}^{-}}\right] \delta_{c}\left(a_{\sigma^{-1}(j)}-a_{\sigma^{-1}(k)}\right) .
$$

Next, consider the following special cases.

$$
\left|I_{\rho}\right|=1, \quad \mathscr{J}_{\rho}(t)=1 .
$$

Then there are indices $i \in\left\{1, \cdots, k_{+}\right\}$and $i_{\delta} \in\left\{1, \cdots, N_{+}\right\}$such that

$$
x_{i_{\delta}}^{+}(t)=x_{i}^{s}-\frac{\delta}{2} \Delta_{i}^{s}+t h^{\prime}\left(p_{i}^{s}\right)+O\left(\exp \left(-|t| r_{\rho}\right)\right), \quad t \rightarrow \delta \infty
$$

where

$$
\Delta_{i}^{s} \equiv\left[\sum_{k \in I_{\rho}^{+}}-\sum_{k \in I_{\rho}^{-}}\right] \delta_{c}\left(p_{i}^{s}-a_{\sigma^{-1}(k)}\right)
$$

(ii) $\quad\left|I_{\rho}\right|=1, \quad \mathscr{J}_{\rho}(t)=-1$.

Then there are indices $j \in\left\{1, \cdots, k_{-}\right\}$and $j_{\delta} \in\left\{1, \cdots, N_{-}\right\}$such that

$$
x_{j_{\delta}}^{-}(t)=x_{j}^{\bar{s}}-\frac{\delta}{2} \Delta_{j}^{\bar{s}}+t h^{\prime}\left(p_{j}^{\bar{s}}\right)+O\left(\exp \left(-|t| r_{\rho}\right)\right), \quad t \rightarrow \delta \infty
$$

where

$$
\Delta_{j}^{\bar{s}} \equiv\left[\sum_{k \in I_{\rho}^{+}}-\sum_{k \in I_{\rho}^{-}}\right] \delta_{c}\left(p_{j}^{\bar{s}}-a_{\sigma^{-1}(k)}\right)
$$


(iii) $\left|I_{\rho}\right|=2, \mathscr{J}_{\rho}(t)=\left[\begin{array}{lc}0 & \exp \left(-i t \eta_{n}\right) \\ \exp \left(i t \eta_{n}\right) & 0\end{array}\right], \eta_{n} \equiv \mu \operatorname{Im} h^{\prime}\left(\frac{1}{2}\left(p_{n}-i \delta_{n}\right)\right), n \in\{1, \cdots, l\}$.

Then there are indices $i_{\delta} \in\left\{1, \cdots, N_{+}\right\}$and $j_{\delta} \in\left\{1, \cdots, N_{-}\right\}$such that

$$
\frac{1}{2}\left[x_{i_{\delta}}^{+}(t)+x_{j_{\delta}}^{-}(t)\right]=x_{n}-\frac{\delta}{2} \Delta_{n}^{c}+t \operatorname{Re} h^{\prime}\left(\frac{1}{2}\left(p_{n}-i \delta_{n}\right)\right)+O\left(\exp \left(-|t| r_{\rho}\right)\right), t \rightarrow \delta \infty
$$

where

$$
\Delta_{n}^{c} \equiv\left[\sum_{k \in I_{\rho}^{+}}-\sum_{k \in I_{\rho}^{-}}\right] \delta_{c}\left(\frac{1}{2}\left(p_{n}-i \delta_{n}\right)-a_{\sigma^{-1}(k)}\right) .
$$

Moreover, when $\delta_{n}>-\mu \mathrm{g}$ one has

$$
\begin{aligned}
\operatorname{sh} \frac{1}{2} \mu\left(x_{i_{\delta}}^{+}(t)-x_{j_{\delta}}^{-}(t)\right) & =\left[\frac{\mu^{2} g^{2}}{\delta_{n}^{2}}-1\right]^{1 / 2} \sin \mu\left(\gamma_{n}-\frac{\delta}{2} \Delta_{n}^{p}+t \operatorname{Im} h^{\prime}\left(\frac{1}{2}\left(p_{n}-i \delta_{n}\right)\right)\right) \\
+ & O\left(\exp \left(-|t| r_{\rho}\right)\right), \quad t \rightarrow \delta \infty
\end{aligned}
$$

where

$$
\Delta_{n}^{p} \equiv\left[\sum_{k \in I_{\rho}^{+}}-\sum_{k \in I_{\rho}^{-}}\right] \delta_{p}\left(\frac{1}{2}\left(p_{n}-i \delta_{n}\right)-a_{\sigma^{-1}(k)}\right)
$$

and when $\delta_{n}=-\mu \mathrm{g}$ one has

$$
x_{i_{\delta}}^{+}(t)-x_{j_{\delta}}^{-}(t)=O\left(\exp \left(-|t| r_{\rho}\right)\right), \quad t \rightarrow \delta \infty .
$$

Proof. Since the eigenvalues of the cluster matrices are bounded away from 0 and $\pm \infty$, it follows from (C15) that

$$
\sum_{j=1}^{\left|I_{\rho}\right|} \ln \left|\alpha_{\rho j}^{\delta}(t)\right|=\ln \left|\operatorname{det} c_{\rho}^{\delta}(t)\right|+t\left|I_{\rho}\right| d_{\rho}+O\left(\exp \left(-|t| r_{\rho}\right)\right), \quad t \rightarrow \delta \infty
$$

Now $\left|c_{\rho}^{\delta}(t)\right|$ equals $(-)^{n(b)+n(\bar{s})}\left|m_{\rho}^{\delta}\right|$, and the latter determinant can be calculated by using (6.27)-(6.30). Doing so, the first assertion readily follows. Then (6.38), (6.41) and (6.44) are obtained by specializing (6.35). (Recall non-real $a_{j}^{\prime} s$ occur in complex-conjugate pairs.)

It remains to prove (6.46) and (6.48). To this end we combine $(\mathrm{C} 15)$ with 
(6.49) to infer

$$
\operatorname{sh} \frac{1}{2} \mu\left(x_{i_{\delta}}^{+}(t)-x_{j_{\delta}}^{-}(t)\right)=\frac{1}{2}\left|\operatorname{det} c_{\rho}^{\delta}(t)\right|^{-1 / 2} \operatorname{Tr} c_{\rho}^{\delta}(t)+O\left(\exp \left(-|t| r_{\rho}\right)\right), \quad t \rightarrow \delta \infty .
$$

Calculating the rhs from (6.27)-(6.30) and (6.43), we now obtain (6.46) and (6.48).

We continue with some remarks on the results just obtained. First, the occurrence of rearrangement collisions already mentioned above is best illustrated by considering a special case: Take $N_{+}=2, N_{-}=1, l=1$, $\frac{1}{2} p_{1}=-p_{1}^{s}>0, \delta_{1}=-\mu g, \quad x_{1}=x_{1}^{s}=0, h(z)=\frac{1}{2} z^{2}$. Then the integral curve describes the following process. For $t \rightarrow-\infty$ one sees a dead breather $x_{2}^{+}(t) \simeq x_{1}^{-}(t)$ at the far left and a soliton $x_{1}^{+}(t)$ at the far right; for $t \rightarrow \infty$ one sees a dead breather $x_{1}^{+}(t) \simeq x_{1}^{-}(t)$ at the far right and a soliton $x_{2}^{+}(t)$ at the far left; for finite times the breather is alive and rearrangement occurs.

Second, it should be noticed that when one would omit the factor $(-)^{k++n-1}$ in the definition (4.3) of $\gamma_{n}$ (so that corresponding terms in (4.26) and (6.16) drop out), then one would obtain an extra factor $(-)^{k_{+}+n-1}$ at the rhs of (6.46).

Third, taking $\delta_{n}>-\mu g$, the shift in the complex breather positions $x_{n} \pm i \gamma_{n}$ due to the scattering at the other clusters can be written in terms of the multi-valued shift function

$$
\delta(z) \equiv \mu^{-1} \ln \left(1+\mu^{2} g^{2} / z^{2}\right), \quad z \neq 0, \pm i \mu g .
$$

Specifically, one has

$$
\Delta\left(x_{n} \pm i \gamma_{n}\right)=\left(\sum_{k \in I_{\rho}^{-}}-\sum_{k \in I_{\rho}^{+}}\right) \delta\left(\frac{1}{2}\left(p_{n} \mp i \delta_{n}\right)-a_{\sigma^{-1}(k)}\right) \quad(\bmod 2 \pi i / \mu) .
$$

Clearly, $\delta_{c}(z)$ equals $\operatorname{Re} \delta(z)$. However, one cannot replace $\delta_{p}$ in (6.47) by $\operatorname{Im} \delta$. Indeed, this renders (6.46) ill defined, since the factor $1 / 2$ multiplying $\Delta_{n}^{p}$ gives rise to sign ambiguities after this substitution.

Fourth, we point out that the above results regarding asymptotics do not include the following items: the asymptotics of $p^{ \pm}(t)$, a description in terms of wave maps, an invariance principle for the latter, and an 'asymptotic constancy' generalization. (See Chapter 4 and Section 5A of I for the pure soliton case.)

We conclude this section by studying the 'dead breathers $\simeq$ solitons' 
phenomenon already described in the Introduction. Thus, we restrict attention to the special case

$$
N_{+}=N_{-}=\frac{1}{2} N \equiv M
$$

and the submanifold

$$
\left.\Omega_{d b} \equiv\left\{x^{+}, x^{-}, p^{+}, p^{-}\right) \in \Omega \mid x^{+}=x^{-}, p^{+},=p^{-}\right\} .
$$

We identify $\Omega_{d b}$ with the manifold

$$
\mathbf{\Omega}^{(M)} \equiv\left\{(q, \theta) \in \boldsymbol{R}^{2 M} \mid q \in G_{M}\right\}
$$

by setting

$$
x^{\delta}=q, \quad p^{\delta}=\frac{1}{2} \theta, \quad \delta=+,-
$$

and equip $\Omega^{(M)}$ with the symplectic form

$$
\omega^{(M)} \equiv \sum_{j=1}^{M} d q_{j} \wedge d \theta_{j}=i^{*}(\omega), \quad i: \Omega^{(M)} \cong \Omega_{d b} \subset \Omega
$$

where $i$ denotes the identification (6.56).

Now consider the Hamiltonian vector field $X \equiv X_{H}$ (with $H$ given by (1.1)) restricted to $\Omega_{d b}$. It is readily seen that $X$ is tangent to $\Omega_{d b}$. Indeed, this amounts to the symmetry properties

$$
X_{j}=X_{M+j}, \quad X_{2 M+j}=X_{3 M+j}, \quad j=1, \cdots, M \quad\left(\text { on } \Omega_{d b}\right)
$$

which are easily verified. Therefore, the flow $\exp (t H)$ leaves $\Omega_{d b}$ invariant and corresponds to the Hamiltonian flow on the symplectic manifold $\left\langle\Omega^{(M)}, \omega^{(M)}\right\rangle$ generated by

$$
H_{r} \equiv i^{*}(H)
$$

In view of (1.1) this Hamiltonian reads

$$
H_{r}(q, \theta)=\frac{1}{4} \sum_{j=1}^{M} \theta_{j}^{2}+2 g^{2} \mu^{2} \sum_{1 \leq j<k \leq M} \frac{1}{\operatorname{sh}^{2} \mu\left(q_{j}-q_{k}\right)}-\frac{1}{4} M g^{2} \mu^{2} .
$$

Thus, its flow equals the pure $M$-soliton flow up to a factor $1 / 2$ (the soliton 
mass is doubled) and a doubling of $\mu$ and $g$.

As a consequence of this state of affairs, we may now infer from Th. 3.2 in I (with $\mu, \rho, N \rightarrow 2 \mu, 2 i g, M$ ) that on $\Omega_{d b}$ one has

$$
\begin{array}{ll}
x_{j}^{\delta}(t) \sim \hat{q}_{j}-\frac{1}{2} \Delta_{j}(\hat{\theta})+\frac{1}{2} t \hat{\theta}_{j} & t \rightarrow \infty \\
p_{j}^{\delta}(t) \sim \frac{1}{2} \hat{\theta}_{j} & t \rightarrow \infty .
\end{array}
$$

Here, $(\hat{q}, \hat{\theta})$ belongs to the action-angle phase space

$$
\hat{\mathbf{\Omega}}^{(M)} \equiv\left\{\left(\hat{q}, \hat{\theta} \in \boldsymbol{R}^{2 M} \mid \hat{\theta} \in G_{M}\right\}, \quad \hat{\omega}^{(M)} \equiv \sum_{j=1}^{M} d \hat{q}_{j} \wedge d \hat{\theta}_{j}\right.
$$

of $\mathrm{I}$ and is given by

$$
(\hat{q}, \hat{\theta})=\Phi_{\mathrm{nr}}(2 \mu, 2 i g ; q, \theta), \quad q \equiv x^{\delta}(0), \quad \theta \equiv 2 p^{\delta}(0)
$$

where $\Phi_{\mathrm{nr}}$ is the map from Th. 2.3 in $\mathrm{I}$; moreover,

$$
\Delta_{j}\left(\theta_{1}, \cdots, \theta_{M}\right)=\left(\sum_{i<j}-\sum_{i>j}\right)(2 \mu)^{-1} \ln \left[1+\frac{16 \mu^{2} g^{2}}{\left(\theta_{j}-\theta_{i}\right)^{2}}\right] .
$$

Now it follows from (6.61), (6.62) that the matrix $L_{t} \equiv L \circ \exp (t H)$ on $\Omega_{d b}$ (with $L$ given by (2.21)) has the $t \rightarrow \infty$ limit

$$
L_{\infty}=\left[\begin{array}{cc}
\mathscr{D} & -\mathscr{C} \\
\mathscr{C} & \mathscr{D}
\end{array}\right], \quad \mathscr{D} \equiv \frac{1}{2} \operatorname{diag}\left(\hat{\theta}_{1}, \cdots, \hat{\theta}_{M}\right), \quad \mathscr{C} \equiv \frac{1}{2} \mu g \mathbf{1}_{M} .
$$

Since the flow is isospectral, this entails

$$
\sigma\left(L_{0}\right)=\sigma\left(L_{\infty}\right)=\left\{\frac{1}{2} \hat{\theta}_{1} \pm \frac{1}{2} i \mu g, \cdots, \frac{1}{2} \hat{\theta}_{M} \pm \frac{1}{2} i \mu g\right\}
$$

so that $\Omega_{d b}$ indeed consists of dead breather states.

More precisely, since $L_{0}$ has $2 M=N$ distinct eigenvalues on the boundary of the strip $S_{\mu g / 2}$, it follows that $\Omega_{d b} \subset \Omega_{s}$ and that $\Phi^{\sharp}$ maps $\Omega_{d b}$ into

$$
\hat{\Omega}_{d b} \equiv\left\{(x, u, p, v) \in \hat{\Omega}_{M}^{\sharp} \mid u_{1}=v_{1}=\cdots=u_{M}=v_{M}=0\right\}
$$


cf. Section 5A. Therefore, we may now invoke Th. 6.2 to deduce

$$
x_{j}^{\delta}(t) \sim x_{j}-\frac{1}{2} \Delta_{j}^{c}+\frac{1}{2} t p_{j} \quad t \rightarrow \infty
$$

where

$$
\Delta_{j}^{c}=\left(\sum_{n<j}-\sum_{n>j}\right)\left[\delta_{c}\left(\frac{1}{2} p_{j}-\frac{1}{2} p_{n}\right)+\delta_{c}\left(\frac{1}{2} p_{j}-\frac{1}{2} p_{n}+i \mu g\right)\right]
$$

cf. (6.44), (6.45). Comparing this to (6.61), we infer successively $p_{j}=\hat{\theta}_{j}, \Delta_{j}^{c}=\Delta_{j}(\hat{\theta})$ (recall (6.32) to check this), $x_{j}=\hat{q}_{j}, j=1, \cdots, M$.

To summarize and extend these findings, we now identify $\hat{\Omega}^{(M)}$ with $\hat{\Omega}_{d b}$ by setting

$$
x=\hat{q}, \quad p=\hat{\theta} .
$$

Denoting this identification by $\hat{i}$, we clearly have

$$
\hat{\omega}^{(M)}=\hat{i}^{*}\left(\hat{\omega}_{M}^{\#}\right), \quad \hat{i}: \hat{\Omega}^{(M)} \cong \hat{\Omega}_{d b} \subset \hat{\Omega}_{M}^{\#}
$$

Theorem 6.3. The map $\Phi^{\sharp}$ restricts to a canonical transformation from $\left\langle\Omega_{d b}, \omega\right\rangle$ onto $\left\langle\hat{\Omega}_{d b}, \hat{\omega}_{M}^{\#}\right\rangle$ which satisfies

$$
\left(\hat{i}^{-1} \circ \Phi^{\#} \circ i\right)(q, \theta)=\Phi_{\mathrm{nr}}(2 \mu, 2 i g ; q, \theta) .
$$

Proof. We have already shown that the diagram

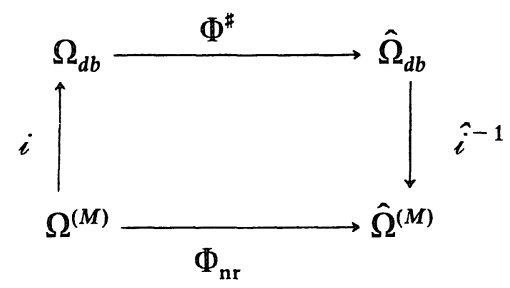

commutes. Thus, it remains to prove $\Phi^{\#}$ maps onto $\hat{\Omega}_{d b}$. But since $\Phi_{\mathrm{nr}}$ is a bijection, this is clear from commutativity.

We close this section with some remarks. First, recall from (6.14)-(6.19) 
that the matrix-valued function $A_{s}$ on $\hat{\Omega}_{M}$ admits an extension to $\hat{\Omega}_{M}^{\#}$. Thus one gets a function $A_{s}(\hat{q}, \hat{\theta})$ on $\hat{\Omega}^{(M)}$ via (6.71). Substituting (6.56) in $A$ (recall (2.6)), one readily deduces

$$
\left|A_{s}(\hat{q}, \hat{\theta})-\alpha \mathbf{1}_{2 M}\right|=\prod_{j=1}^{M}\left(\alpha^{2}-\exp 2 \mu q_{j}\right) .
$$

Since the map $(q, \theta) \mapsto(\hat{q}, \hat{\theta})$ is canonical, it follows that the symmetric functions of $A_{s}(\hat{q}, \hat{\theta})$ Poisson commute. In point of fact, these functions can be explicitly found: from (6.14)-(6.19) one obtains

$$
A_{s}(\hat{q}, \hat{\theta})=\left[\begin{array}{lc}
0 & \operatorname{diag}\left(c_{1}, \cdots, c_{M}\right) \\
\frac{1}{2} C(0,2 \mu,-2 g ; \hat{\theta}, \hat{\theta}) & 0
\end{array}\right]
$$

where $C$ is given by $(\mathrm{B} 1)$ and where

$$
c_{j}=2 \exp \left(2 \mu \hat{q}_{j}\right) \prod_{k \neq j}\left[1+\frac{16 \mu^{2} g^{2}}{\left(\hat{\theta}_{j}-\hat{\theta}_{k}\right)^{2}}\right]^{1 / 2} .
$$

From Cauchy's identity (B4) it then follows that the functions $S_{2 k}\left(A_{s}\right)$ coincide with the functions $S_{k}\left(\mathrm{I}_{\text {rel }}\right)$ of I with $\mu \rightarrow 2 \mu, \rho \rightarrow 2 i g, N \rightarrow M$.

Second, when we substitute (6.56) in the Lax matrix (2.21), yielding a $2 M \times 2 M$ matrix $L^{(2)}(q, \theta)$, then the symmetric functions of $L^{(2)}$ are real-valued and commute. Indeed, by virtue of (6.67) one has

$$
\left|L^{(2)}(q, \theta)-\lambda \mathbf{1}_{2 M}\right|=\prod_{j=1}^{M}\left[\left(\lambda-\frac{1}{2} \hat{\theta}_{j}\right)^{2}+\frac{1}{4} \mu^{2} g^{2}\right]
$$

which proves the assertion.

Third, using $k^{\text {th }}$ roots of unity the fusion procedure can be generalized to obtain $k M \times k M$ matrices $L^{(k)}$ on $\Omega^{(M)}$ with real-valued commuting symmetric functions, the function $\frac{1}{2} k \operatorname{Tr} L^{(k)}(q, \theta)$ (minus an irrelevant constant) being equal to the Hamiltonian $H$ from Th. 3.2 in I, with $\mu, \rho, N \rightarrow k \mu$, kig, $M$. This entails remarkable restriction properties of the holomorphic function $R$ from Chapter 3 , which generalize (6.73).

Finally, we point out that the $C_{l}$ and $B C_{l}$ reduction described in Th. 5.2 of I generalizes to the $\tilde{\Pi}_{n r}$ case. The point is, that when one requires that 
the two particles at equal distance from the origin have the same charge, then the vector field $X_{H}$ is again tangent to (the obvious generalization of) $\Omega^{e}$ for $N=2 l$ and to $\Omega^{0}$ for $N=2 l+1$, resp.

\section{B. The Case $\tilde{\Pi}_{\mathrm{rel}}(\tau<\pi / 2)$}

Just as in previous chapters, provided that $\tau \in(0, \pi / 2)$, the reasoning for the $\tilde{\Pi}_{n r}$ case can be followed to a considerable extent. In particular, we can consider the same class $\mathscr{C}$ of entire functions $h$, but now (6.1) should be replaced by

$$
H_{h} \equiv \operatorname{Tr} h\left(\beta^{-1} \ln L\right), \quad h \in \mathscr{C} .
$$

Here, the matrix $\ln L$ can (and will) be defined by the (Riesz-Dunford) functional calculus. (Recall $\sigma(L)$ belongs to the open right half plane.) Then $\ln L$ is real-analytic on $\Omega$, so that $H_{h}$ is real-analytic on $\Omega$, too. Moreover, on $\Omega_{s}$ one has

$$
\beta^{-1} \ln L \sim \operatorname{diag}\left(a_{1}, \cdots, a_{N}\right)
$$

cf. (5.72). Therefore, $H_{h}$ is real-valued on $\Omega_{s}$ and hence on $\Omega$ as well.

Clearly, the Hamiltonian $H$ given by (1.2) arises when one takes $h(z) \equiv \beta^{-1} \exp \beta z$, cf. (2.73). In the present case however, completeness of the $H$ flow is not immediate, since the functions $\exp \beta p_{k}^{\delta}$ in (1.2) are not bounded away from 0 on $\Omega$. But if we start from $h(z) \equiv \operatorname{ch} \beta z$, then we obtain the Hamiltonian $P_{0}$ given by (5.70), and completeness of the $P_{0}$ flow is again obvious. Now (6.2) still holds, and the definition (6.3) then leads once again to (6.4). Therefore, all Hamiltonians $H_{h}, h \in \mathscr{C}$, commute on $\Omega$, and from (6.5) and (6.6) one infers that their flows are complete on $\Omega_{r}$. Setting

$$
A_{h}(t, P) \equiv A(P) \exp \left(t \mu h^{\prime}\left(\beta^{-1} \ln L(P)\right)\right)
$$

we are now prepared for the analog of Th. 6.1 .

Theorem 6.4. For $\tau \in(0, \pi / 2)$ the assertions of Th. 6.1 hold true.

Proof. The proof of Th. 6.1 can easily be adapted. Specifically, the role of (2.47), (2.48) is played by (2.84), (2.85), and (4.1)-(4.3) should be modified as detailed in the first paragraph of Section 4B. Substituting 


$$
\tilde{L}(\hat{P}) \rightarrow \beta^{-1} \ln \tilde{L}(\hat{P})
$$

in (6.12), the reasoning now applies verbatim.

Continuing as in the previous case, the definition (6.14) leads to (6.13) with the replacement (6.82). Then (6.15) holds true with 0 replaced by $\beta$, and with $S$ defined via (6.16), (6.17) with the following changes: in (6.16) one should substitute

$$
\begin{aligned}
& \mu x_{i}^{s} \rightarrow \mu x_{i}^{s}+\beta p_{i}^{s}, \quad i=1, \cdots, k_{+} \\
& \mu\left(x_{n}+i \gamma_{n}\right) \rightarrow \mu\left(x_{n}+i \gamma_{n}\right)+\frac{1}{2} \beta\left(p_{n}-i \delta_{n}\right), \quad n=1, \cdots, l \\
& \mu x_{j}^{\bar{s}} \rightarrow \mu x_{j}^{\bar{s}}+\beta p_{j}^{\bar{s}}, \quad j=1, \cdots, k_{-}
\end{aligned}
$$

and $\mathscr{V}_{k}$ is now given by (3.57). Then (6.18) follows once more, with (6.19) replaced by

$$
c_{n} \equiv 2 \cos \tau \exp \left(2 \mu x_{n}+\frac{1}{2} \beta p_{n}\right) \prod_{j \neq k_{+}+n, k_{+}+l+n}\left|1+\frac{\sin ^{2} \tau}{\operatorname{sh}^{2} \frac{1}{2} \beta\left(a_{k_{+}+n}-a_{j}\right)}\right| .
$$

Next, we fix $h \in \mathscr{C}$ and proceed as before: (6.20)-(6.26) apply again, and in (6.27) we need only replace 0 by $\beta$. Lemma B2 then yields (6.28), with (6.29) generalized to

$$
g_{j}^{\delta} \equiv \prod_{m \in I_{\rho}^{\delta}} \frac{\operatorname{sh} \frac{1}{2} \beta\left(p_{j}-p_{m}\right)}{\operatorname{sh} \frac{1}{2} \beta\left(p_{j}-\bar{p}_{m}-i \mu g\right)}, \quad j \in I_{\rho} .
$$

Then (6.30) applies again. To state the analog of Th. 6.2, we replace (6.32)-(6.34) by the definitions

$$
\begin{aligned}
& \delta_{c}: S_{\mu g}^{c l} \backslash\{0, \pm i \mu g\} \rightarrow R, \quad z \mapsto \mu^{-1} \ln \left|1+\frac{\sin ^{2} \tau}{\operatorname{sh}^{2} \frac{1}{2} \beta z}\right|, \quad \tau \equiv \frac{1}{2} \beta \mu g \\
& \delta_{p}: S_{\mu g} \backslash\{0\} \rightarrow 2 \mu^{-1}(-\pi, \pi], \quad z \mapsto 2 \mu^{-1} \arg \left[\frac{\left[\operatorname{sh}^{2} \frac{1}{2} \beta z+\sin ^{2} \tau\right]^{1 / 2}}{\operatorname{sh} \frac{1}{2} \beta z}\right]
\end{aligned}
$$




$$
\operatorname{Re}\left[\operatorname{sh}^{2} \frac{1}{2} \beta z+\sin ^{2} \tau\right]^{1 / 2}>0, \quad z \in S_{\mu g}
$$

Theorem 6.5. For $\tau \in(0, \pi / 2)$ the assertions of Th. 6.2 hold true, with $\delta_{c}$ and $\delta_{p}$ given by (6.86) and (6.87), and with the replacement

$$
\left[\frac{\mu^{2} g^{2}}{\delta_{n}^{2}}-1\right]^{1 / 2} \rightarrow\left[\frac{\sin ^{2} \tau}{\sin ^{2} \frac{1}{2} \beta \delta_{n}}-1\right]^{1 / 2}
$$

in (6.46).

Proof. Since (6.49) and (6.50) are still valid, we can proceed as before, using the generalization (6.85) of (6.29).

The remarks after Th. 6.2 apply with obvious changes. In particular, (6.51) should be replaced by

$$
\delta(z) \equiv \mu^{-1} \ln \left[1+\frac{\sin ^{2} \tau}{\operatorname{sh}^{2} \frac{1}{2} \beta z}\right], \quad z \neq 2 \pi i k \beta^{-1}, \pm i \mu g+2 \pi i k \beta^{-1}, \quad k \in Z
$$

and then (6.52) holds true again.

We continue by generalizing the reduction result Th. 6.3, starting again from (6.53)-(6.57). With $H$ given by (1.2), the vector field $X \equiv X_{H}$ again has the symmetry properties (6.58). Thus, the $H$ flow restricts to $\Omega_{d b}$ and can be identified with the flow on $\Omega^{(M)}$ generated by

$$
H_{r}(q, \theta)=\left(\frac{1}{2} \beta\right)^{-1} \cos \tau \sum_{j=1}^{M} \exp \left(\frac{1}{2} \beta \theta_{j}\right) \prod_{k \neq j}\left[1+\frac{\sin ^{2} 2 \tau}{\operatorname{sh}^{2} \mu\left(q_{j}-q_{k}\right)}\right]^{1 / 2} \text {. }
$$

(Indeed, $H_{r}$ is related to $H$ via (6.59).) Hence one obtains the pure $M$-soliton flow of Th. 3.4 in I, with $\beta, \mu, z=i \tau$ replaced by $\frac{1}{2} \beta, 2 \mu, 2 z$ and with a scale factor $\cos \tau$. (Physically speaking, the latter corresponds to the mass defect 
of the relativistic bound state.) From I 1.c. it then follows that the asymptotics of the $H$ flow on $\Omega_{d b}$ is given by

$$
x_{j}^{\delta}(t) \sim \hat{q}_{j}-\frac{1}{2} \Delta_{j}(\hat{\theta})+t \cos \tau \exp \frac{1}{2} \beta \hat{\theta}_{j} \quad t \rightarrow \infty
$$

and by $(6.62)$, with $(\hat{q}, \hat{\theta})$ in the phase space $(6.63)$ and with $(6.64),(6.65)$ replaced by

$$
\begin{aligned}
& (\hat{q}, \hat{\theta})=\Phi_{\mathrm{rel}}\left(\frac{1}{2} \beta, 2 \mu, i \beta \mu g ; q, \theta\right), \quad q \equiv x^{\delta}(0), \quad \theta \equiv 2 p^{\delta}(0) \\
& \Delta_{j}\left(\theta_{1}, \cdots, \theta_{M}\right)=\left(\sum_{i<j}-\sum_{i>j}\right)(2 \mu)^{-1} \ln \left[1+\frac{\sin ^{2} 2 \tau}{\operatorname{sh}^{2} \frac{1}{4} \beta\left(\theta_{j}-\theta_{i}\right)}\right] .
\end{aligned}
$$

Using (6.92), (6.62) we now get as the analog of (6.66)

$$
L_{\infty}=\left[\begin{array}{rr}
\cos \tau \mathscr{D} & -\sin \tau \mathscr{D} \\
\sin \tau \mathscr{D} & \cos \tau \mathscr{D}
\end{array}\right], \quad \mathscr{D} \equiv \operatorname{diag}\left(\exp \frac{1}{2} \beta \hat{\theta}_{1}, \cdots, \exp \frac{1}{2} \beta \hat{\theta}_{M}\right)
$$

(recall (2.70)), so that

$$
\sigma\left(L_{\infty}\right)=\left\{\exp \frac{1}{2} \beta\left(\hat{\theta}_{1} \pm i \mu g\right), \cdots, \exp \frac{1}{2} \beta\left(\hat{\theta}_{M} \pm i \mu g\right)\right\}
$$

Therefore, one has again $\Omega_{d b} \subset \Omega_{s}$, $\Phi^{\sharp}\left(\Omega_{d b}\right) \subset \hat{\Omega}_{d b}$, cf. (6.68). From Th. 6.5 we then obtain

$$
x_{j}^{\delta}(t) \sim x_{j}-\frac{1}{2} \Delta_{j}^{c}+t \cos \tau \exp \frac{1}{2} \beta p_{j} \quad t \rightarrow \infty
$$

with $\Delta_{j}^{c}$ given by (6.70), (6.86), and comparing this to (6.92) we deduce again $p_{j}=\hat{\theta}_{j}, \Delta_{j}^{c}=\Delta_{j}(\hat{\theta}), x_{j}=\hat{q}_{j}$. Defining $\hat{i}$ by (6.71) and recalling (6.72), we are now prepared for the analog of Th. 6.3.

Theorem 6.6. With (6.73) replaced by

$$
\left(\hat{i}^{-1} \circ \Phi^{\#} \circ i\right)(q, \theta)=\Phi_{\mathrm{rel}}\left(\frac{1}{2} \beta, 2 \mu, i \beta \mu g ; q, \theta\right)
$$


the assertion of Th. 6.3 holds true.

Proof. After replacing $\Phi_{\mathrm{nr}}$ by $\Phi_{\text {rel }}$ the proof of Th. 6.3 applies verbatim.

The remarks after Th. 6.3 can be adapted with minor changes. First, (6.75) is still valid, and (6.76), (6.77) should be replaced by

$$
\begin{aligned}
& A_{s}(\hat{q}, \hat{\theta})=\left[\begin{array}{cc}
0 & \operatorname{diag}\left(c_{1}, \cdots, c_{M}\right) \\
(2 \cos \tau)^{-1} C\left(\frac{1}{2} \beta, 2 \mu,-2 g ; \hat{\theta}, \hat{\theta}\right) & 0
\end{array}\right] \\
& c_{j}=2 \cos \tau \exp \left(2 \mu \hat{q}_{j}+\frac{1}{2} \beta \hat{\theta}\right) \prod_{k \neq j}\left[1+\frac{\sin ^{2} 2 \tau}{\operatorname{sh}^{2} \frac{1}{4} \beta\left(\hat{\theta}_{j}-\hat{\theta}_{k}\right)}\right]^{1 / 2}
\end{aligned}
$$

In the present case, (B4) entails equality of the functions $S_{2 k}\left(A_{s}\right)$ and the functions $S_{k}\left(\mathrm{II}_{\mathrm{rel}}\right)$ of I with the replacements $\beta \rightarrow \beta / 2, \mu \rightarrow 2 \mu, z \rightarrow i \beta \mu g, N \rightarrow M$.

Second, in view of $(6.96)$ the analog of $(6.78)$ reads

$$
\left|L^{(2)}(q, \theta)-\lambda \mathbb{1}_{2 M}\right|=\prod_{j=1}^{M}\left[\lambda^{2}-2 \lambda \cos \tau \exp \left(\frac{1}{2} \beta \hat{\theta}_{j}\right)+\exp \left(\beta \hat{\theta}_{j}\right)\right]
$$

Third, assuming $\tau \in(0, \pi / 2)$ satisfies $\sin k \tau \neq 0, k$-fold fusion can again be performed, the function $\sin k \tau(k \sin \tau)^{-1} \operatorname{Tr} L^{(k)}$ being equal to the Hamiltonian $H$ from Th. 3.4 in I with $\beta, \mu, z, N \rightarrow k^{-1} \beta, k \mu, \frac{1}{2} i k \beta \mu g, M$.

Finally, the $C_{l} / B C_{l}$ reduction remark applies again, with $X_{H}$ replaced by $X_{P_{0}}$.

$$
\text { 6C. The Case } \tilde{\Pi}_{\text {rel }}(\tau=\pi / 2)
$$

In this section we study the singular case $\beta \mu g=\pi$. Taking $P \in \Omega^{b}$, one can again define $\ln L(P)$ by the functional calculus. Then (6.80) holds true on $\Omega_{s}^{b}$, and for any $h \in \mathscr{C}$ the definition (6.79) yields a real-valued real-analytic Hamiltonian $H_{h}$ on $\Omega^{b}$. Defining $\hat{H}_{h, l}$ on $\hat{\Omega}_{l}^{b}$ by (6.3), it now follows from (6.79), (6.80) and (6.2) (with $\Omega \rightarrow \Omega^{b}$ ) that (6.4) holds true for any $\hat{P} \in \hat{\Omega}_{l}^{b}$. Arguing as before, this entails that all Hamiltonians $H_{h}$ commute on $\Omega^{b}$.

However, the $H_{h}$ flow is not complete on $\Omega^{b}$ whenever $h(z)$ depends 
nonlinearly on $z$, as will be clear from what follows. (Of course, $\Omega^{b}$ is left invariant when $h(z)=a_{0}+a_{1} z$.) For instance, it is easily seen that any integral curve of

$$
P_{0}=\left(\operatorname{ch} \beta p^{+}+\operatorname{ch} \beta p^{-}\right)\left|\operatorname{th} \frac{1}{2} \mu\left(x^{+}-x^{-}\right)\right| \quad\left(N_{+}=N_{-}=1\right)
$$

yields $x^{+}-x^{-} \rightarrow 0,\left|p^{+}\right|,\left|p^{-}\right| \rightarrow \infty$ in finite time.

When one is willing to ignore sets of measure zero, there is a quite simple solution to this problem, which will now be detailed. Fixing $P \in \Omega_{l}^{b}$ with image $\hat{P} \in \hat{\Omega}_{l}^{b}$ under $\Phi_{l}$, we define a curve $\hat{P}(t)$ in $\hat{\Omega}_{l}$ via the $r h s$ of (6.6). Then the set $C_{h . P}$ of times for which this curve intersects $\hat{\Omega}_{l} \backslash \hat{\Omega}_{l}^{b}$ does not contain an interval around $t=0$. We claim that $C_{h . P}$ is actually discrete.

Indeed, lifting the curve to a curve $\tilde{\hat{P}}(t)$ in the covering manifold $\tilde{\hat{\Omega}}_{l} \subset \mathscr{H} \subset C^{2 N}$, the set consists of those times at which $\tilde{\hat{P}}(t) \in \mathcal{N}_{\mathscr{F}}$, cf. (4.48), (3.64). Since the function $t \mapsto \mathscr{F}(\tilde{\hat{P}}(t))$ is real-analytic on $\mathbb{R}$, existence of a limit point in $C_{h, P}$ would entail $\tilde{P}(t) \in \mathscr{N}_{\mathscr{F}}$ for any $t \in \mathbb{R}$, a contradiction. Thus our claim follows.

Obviously, for any connected component $I$ of $R \backslash C_{h, P}$ the curve $I \rightarrow \Omega^{b}$, $t \mapsto \mathscr{E}_{I}(\hat{P}(t)) \equiv \exp \left(t H_{n}\right)(P)$ is a maximal integral curve of $H_{h}$. Thus we obtain piecewise integral curves going through any $P \in \Omega_{s}^{b}$. In the Koopman picture of classical mechanics (cf. e.g. [19]), this piecewise definition of $\exp \left(t H_{h}\right)(P)$, $P \in \Omega_{s}^{b}$, yields the pointwise action of a strongly continuous 1-parameter group of measure-preserving (hence unitary) transformations on $L^{2}(\Omega)$. Indeed, the $r h s$ of (6.6) gives rise to such a group $\hat{U}(t)$ on $L^{2}(\hat{\Omega})$, and the canonical map $\mathscr{E}$ from $\hat{\Omega}^{b}$ onto $\Omega_{s}^{b}$ gives rise to an isometry $W$ from $L^{2}(\hat{\Omega})$ onto $L^{2}(\Omega)$, since $\hat{\Omega} \backslash \hat{\Omega}^{b}$ and $\Omega \backslash \Omega_{s}^{b}$ have measure zero. Thus the image group $U(t)$ on $L^{2}(\Omega)$ has the asserted properties, entailing completeness in the functional analytic sense.

However, this solution leaves much to be desired. We shall present a more satisfactory remedy at the end of this section, after having assembled extensive information that is of interest in its own right. First of all, we shall show that there exists a clear-cut method to piece together maximal integral curves for any initial point in the exceptional set $\Omega_{e}^{b}$ too. To this end we shall make use of the matrix-valued function $A_{h}(t, P)$, cf. (6.81). We begin by noting that for $P \in \Omega_{l}^{p}$ and $t \in R \backslash C_{h . P}$ one has

$$
A_{h}(t, P) \sim \tilde{A}(\hat{P}) \exp \left(t \mu h^{\prime}\left(\beta^{-1} \ln \tilde{L}(\hat{P})\right)\right)=\tilde{A}\left(\exp \left(t \hat{H}_{h . l}\right)(\hat{P})\right) \sim A\left(\exp \left(t H_{h}\right)(P)\right)
$$


on account of $(6.2)-(6.6)$. Therefore, $A_{h}(t, P)$ has simple and real spectrum and its eigenvalues can be ordered according to (6.8); moreover, the position part of $\exp \left(t H_{h}\right)(P)$ is given by (6.9).

Now consider the general case $P \in \Omega^{b}$. Then the argument below (6.12) still applies, so that (6.8) and (6.9) hold true for small $|t|$. Next, let us introduce

$$
\mathscr{F}(t, P) \equiv \prod_{j<k}\left[\alpha_{j}(t)+\alpha_{k}(t)\right]^{2}
$$

where $\alpha_{1}(t), \cdots, \alpha_{N}(t)$ are the roots of $\left|A_{h}(t, P)-\alpha \mathbf{1}_{N}\right|$. Since the rhs is invariant under permutations, $\mathscr{F}$ is well defined and can be written as a polynomial in the symmetric functions of $A_{h}(t, P)$. Thus $\mathscr{F}$ is real-analytic on $\boldsymbol{R} \times \Omega^{b}$; moreover, $\mathscr{F}$ is non-zero and real-valued for $|t|$ small enough. But then it follows that $\mathscr{F}$ is real-valued on $\boldsymbol{R}$ and that the set

$$
C_{h, P} \equiv\{t \in \boldsymbol{R} \mid \mathscr{F}(t, P)=0\}
$$

is discrete.

Of course, for $P \in \Omega_{s}^{b}$ the set $C_{h, P}$ coincides with the collision set already encuntered. Fixing $P \in \Omega_{e}^{b}$ and a connected component $I$ of $C_{h . P}$ not containing $t=0$, the question is now whether the spectrum of $A_{h}(t, P)$ is again related via (6.8) and (6.9) to an $H_{h}$ integral curve on $I$ that is uniquely determined by $P$. (We have already seen that this is indeed the case when $0 \in I$.)

To answer this question in the affirmative, we begin by recalling $\Omega_{s}^{b}$ is dense in $\Omega^{b}$. To exploit this, we fix a closed ball with center $P$ whose radius $r$ is chosen small enough so that the ball belongs to $\Omega^{b}$, and we consider the line segments connecting $P$ to the surface of the ball. Recalling that $\Omega_{e}^{b}$ is the zero locus of the discriminant function $D$, which is real-analytic on $\Omega^{\text {b }}$ (cf. (4.42)), it follows that a line segment either belongs to $\Omega_{e}^{b}$ or meets $\Omega_{e}^{b}$ in a finite set. Since $\Omega_{e}^{b}$ is nowhere dense, there exists a segment for which the second possibility applies. Therefore, there exists a segment $S:(0, \delta) \rightarrow \Omega^{b}$, $s \mapsto P_{s}$ of length $\delta \leqslant r$ such that $P_{s} \rightarrow P$ as $s \rightarrow 0$ and such that $S$ does not meet $\Omega_{e}^{b}$. Now $\Omega_{e}^{b}$ is the complement of the connected components of $\Omega_{s}^{b}$, so it follows that $S$ belongs to a component of $\Omega_{l}^{b}$ for some $l \in\{0, \cdots, m\}$.

Next, we fix a compact interval $K \subset I$ and note $\mathscr{F}\left(t, P_{s}\right) \neq 0$ for $t \in K$ and $s \leqslant \epsilon \leqslant \delta$. (Indeed, $\mathscr{F}(t, P)$ is non-zero on $I$ and hence stays a finite distance from 0 on $K$. Since $P_{s} \rightarrow P$ as $s \rightarrow 0$ and $\mathscr{F}(\cdot ;)$ is continuous on $R \times \Omega^{b}$, this also holds true for $\mathscr{F}\left(t, P_{s}\right)$, provided $s$ is small enough.) Consequently, for 
any $s \in(0, \epsilon]$ the piecewise integral curve $\exp \left(t H_{h}\right)\left(P_{s}\right) \equiv P_{s}(t)$ is defined on $K$. Our aim is now to show that the limit $P(t)$ of $P_{s}(t)$ for $s \rightarrow 0$ exists for any $t \in K$ and yields an integral curve $K \rightarrow \Omega^{b}, t \mapsto P(t)$.

We begin by noting that there is a finite lower bound on the quantities $\left|\alpha_{j s}(t)+\alpha_{k s}(t)\right|$ for $(s, t) \in(0, \epsilon] \times K$. (Indeed, all eigenvalues $\alpha_{l s}(t)$ of $A_{h}\left(t, P_{s}\right)$ remain bounded and $\mathscr{F}\left(t, P_{s}\right)$ stays away from 0 .) Using constancy of $\operatorname{Tr}\left(L+L^{-1}\right)$ along integral curves in a by now familiar way, it follows that there is a finite lower bound on the quantities $\left|\alpha_{j s}(t)-\alpha_{k s}(t)\right|$ and a finite upper bound on the norm of the momentum part $p_{s}(t)$ of $P_{s}(t)$ for $(s, t) \in(0, \epsilon] \times K$. Therefore, the position part $x_{s}(t)$ of $P_{s}(t)$ does converge to a position vector $x(t)$ in the configuration space of $\Omega^{b}$ for any $t \in K$.

Now $p_{s}(t)$ remains bounded for $t \in K$, so the set $\Lambda_{t}$ of limit points of $p_{s}(t)$ as $s \rightarrow 0$ is non-empty. We claim that $\left|\Lambda_{t}\right|=1$, i.e., that $p_{s}(t)$ has a limit $p(t) \in \boldsymbol{R}^{N}$ as $s \rightarrow 0$. Accepting this for a moment, let us first show that the limit $P(t) \equiv(x(t), p(t))$ of $P_{s}(t)$ for $s \rightarrow 0$ and $t \in K$ is in fact an integral curve of $H_{h}$, as advertised above. To this end we exploit Hamilton's equation $\dot{P}_{s}(t)=\left(\mathscr{S} \nabla H_{h}\right)$ $\left(P_{s}(t)\right)$, where the $r h s$ is the symplectic gradient, cf. (3.31). Since $H_{h}$ is real-analytic on $\Omega^{b}$, its gradient remains bounded on compacts of $\Omega^{b}$. Therefore, when we integrate the Hamilton equation over an interval belonging to $K$, the integrand at the $r h s$ remains bounded as $s \rightarrow 0$. Since it converges pointwise, we may use the domainated convergence theorem and the fundamental theorem of calculus to deduce that $P(t), t \in K$, is an integral curve of $H_{h}$.

Next, we prove our claim $\left|\Lambda_{t}\right|=1$. First, we note that $p_{s}(t), t \in K$, is $\cdots$ continuous on $(0, \epsilon]$. (Indeed, $p_{s}(0)$ is continuous, so this follows from inspection of (6.6) and continuity of $\Phi_{l}$ and $\mathscr{E}_{l}$.) Therefore, the limit set $\Lambda_{t}$ must be connected. So if $\left|\Lambda_{t}\right|>1$, then $\Lambda_{t}$ is not a finite set. We now continue by ruling out $\left|\Lambda_{t}\right|=\infty$.

We first observe that the spectrum of the Lax matrix in points of the form

$$
(x, p), \quad x=\lim _{s \rightarrow 0} x_{s}(t), \quad p \in \Lambda_{t}, \quad t \in K
$$

equals $\sigma(L(P))$. (To see this, use $\sigma\left(L\left(P_{s}(0)\right)\right)=\sigma\left(L\left(P_{s}(t)\right)\right)$.) $\quad$ Now the impossibility of $\left|\Lambda_{t}\right|=\infty$ follows from a Fact that is of some interest by itself, viz.: For a fixed $(x, \tilde{p}) \in \Omega^{b}$, there are at most finitely many $p$ such that $\sigma(L(x, p))=\sigma(L(x, \tilde{p}))$.

To prove this, we write 


$$
L(x, p) \sim \operatorname{diag}\left(z_{1}, \cdots, z_{N}\right) \widetilde{C}(x) \equiv M(z, x), \quad z_{j} \equiv \exp \left(\beta p_{j}\right)
$$

cf. (2.70). Next, we consider the zero locus $V \subset C^{N}$ of the polynomials

$$
S_{k}(M(z, x))-S_{k}(M(\tilde{z}, x)), \quad k=1, \cdots, N, \quad \tilde{z}_{j} \equiv \exp \left(\beta \tilde{p}_{j}\right)
$$

in $z_{1}, \cdots, z_{N}$. Since $V$ is an affine algebraic variety, $V$ is either a finite point set (containing in particular $\tilde{z}$ ) or $V$ is unbounded. Suppose $V$ is unbounded. Then there exists a sequence $z^{(n)} \in V$ and $k \geqslant 1$ indices $i_{1}, \cdots, i_{k}$ such that $z_{i_{1}}^{(n)}, \cdots, z_{i_{k}}^{(n)}$ diverge for $n \rightarrow \infty$, whereas the remaining $z_{i}$ stay bounded. Since $S_{k}\left(M\left(z^{(n)}, x\right)\right)$ equals $S_{k}(M(\tilde{z}, x))$, we may then deduce

$$
0=\lim _{n \rightarrow \infty} S_{k}\left(M\left(z^{(n)}, x\right)\right) / z_{i_{1}}^{(n)} \cdots z_{i_{k}}^{(n)}=\tilde{C}\left(i_{1}, \cdots, i_{k}\right)
$$

where the rhs denotes the relevant principal minor of $\tilde{C}(x)$, cf. (6.107). But since $(x, \tilde{p}) \in \Omega^{b}$, this minor does not vanish, a contradiction. Thus, the Fact is now proved. (It is easy to adapt this argument to the $\tilde{\mathbb{I}}_{\mathrm{rel}}(\tau<\pi / 2)$ and $\tilde{\mathbb{I}}_{n r}$ systems, with $\Omega^{b}$ replaced by $\Omega$. Thus the Fact is a fact for these systems, too.)

Where do we stand? We have shown that for any $P \in \Omega_{e}^{b}$ and $h \in \mathscr{C}$ one can piece together maximal integral curves of $H_{h}$ whose position part is related to $\sigma\left(A_{h}(t, P)\right), t \in \mathbb{R} \backslash C_{h, P}$, via (6.8) and (6.9). In order to study the uniqueness of this procedure (and for later purposes) it is important to extend the reasoning to $n$-parameter flows. Thus, let $P \in \Omega^{b}, h_{1}, \cdots, h_{n} \in \mathscr{C}$ and $i=\left(t_{1}, \cdots, t_{n}\right) \in \mathbb{R}^{n}$. Then the above arguments and the equations (6.103)-(6.105) have straightforward generalizations. In particular, $\exp \left(\sum_{j=1}^{n} t_{j} H_{h_{j}}\right)(P)$ has an obvious meaning for $P \in \Omega_{s}^{b}$ and $t \notin C_{h_{1}, \cdots h_{n} . P}$, and then the above approximation argument shows that through any $P$ in the exceptional set, too, there passes a piecewise orbit $P(t)$ (locally a submanifold of $\Omega^{b}$ with dimension $\leqslant \min (N, n)$ ), whose configuration space projection is uniquely determined by $\sigma\left(A_{h_{1}, \cdots h_{n}}(t, P)\right)$, $t \in \mathbb{R}^{n} \backslash C_{h_{1}, \cdots h_{n} . P}$, via (6.8) and (6.9).

Returning to a fixed $h \in \mathscr{C}$, we now discuss the uniqueness of the piecing. Here we hit a snag: A priori, there might be two different maximal curves having equal $\sigma(L)$ and equal position parts. We believe that this cannot happen for $h(z)$ nonlinear, but we are unable to rule out this contingency. Fortunately, we do not need to know this to show that to all intents and purposes the above piecing is unique.

To be specific, we shall prove that whenever a sequence $P_{n} \in \Omega_{s}^{p}$ converges to $P \in \Omega_{e}^{b}$ as $n \rightarrow \infty$, the piecewise integral curves $\exp \left(t H_{h}\right)\left(P_{n}\right)$ converge to 
the piecewise integral curve through $P$ defined via the above line segment $S$. Arguing as before, this is plain for the position parts. Consider now the limit set $\omega_{t}, t \in K$, for the momentum parts $p_{n}(t)$ as $n \rightarrow \infty$. This set is non-empty, but not connected, a priori. Suppose $\omega_{t} \neq \Lambda_{l_{t}}=\{p(t)\}$, where $p(t)$ is obtained via the segment $S$. Then there exists $\tilde{p}(t) \in \omega_{t}$ such that $\tilde{p}(t) \neq p(t)$, and a subsequence converging to $\tilde{p}(t)$.

Next, we compare the piecewise orbits $\exp \left(t_{1} H_{h}+t_{2} H_{h_{2}}\right)(P), h_{2}(z) \equiv \beta^{-1}$ $\exp (\beta z)$, as obtained via this subsequence and as obtained via $S$. Of course, these have equal position parts and they coincide on the component of $\boldsymbol{R}^{2} \backslash C_{h, h_{2} . P}$ containing the origin. However, by assumption one has $(t, 0) \notin C_{h, h_{2}, P}$ and $\tilde{p}(t, 0) \neq p(t, 0)$. That is, near $(t, 0)$ the position vectors are equal, but the momentum vectors are not. This yields the desired contradiction, as $\partial_{t_{1}} x_{j}=\exp \left(\beta \tilde{p}_{j}\right) V_{j} \neq \exp \left(\beta p_{j}\right) V_{j}=\partial_{t_{2}} x_{j}$ for at least one $j$.

Now that we have proved uniqueness of the integral curves $\boldsymbol{R} \backslash C_{h, P} \rightarrow \Omega^{b}$, $t \mapsto \exp \left(t H_{h}\right)(P)$, we can also show that the curves have a continuous dependence on the initial point. Specifically, we assert that whenever a sequence $P_{n} \in \Omega^{b}$ converges to $P \in \Omega^{b}$ as $n \rightarrow \infty$, the corresponding curves converge, too. To prove this, one need only repeat the previous argument: It applies almost verbatim. (Of course, for $P \in \Omega_{s}^{b}$ this property is evident; the non-trivial case is sequences in $\Omega_{e}^{b}$.)

There is yet another way to obtain the above piecewise integral curves that is illuminating in itself and that will be quite useful shortly. This consists in exploiting Th. 6.4 and continuity in $g$. More specifically, fixing $P \in \Omega^{b}$ and $h \in \mathscr{C}$, the limit of the global integral curve $R \rightarrow \Omega, t \mapsto \exp \left(t H_{h}(g)\right)(P)$ as $g \uparrow \pi / \mu \beta$ exists provided $t \in R \backslash C_{h, P}$, and coincides with the piecewise integral curve of $H_{h}=H_{h}(\pi / \mu \beta)$ defined above. Again, the proof of this characterization is simply a variation on previous arguments: Convergence of the position part follows from continuity of $A_{h}(t, P)$ in $g$, and then the momentum part must 'come along'. (To bypass the above-mentioned snag, the argument should be generalized again to the $n$-parameter case, which is straightforward.)

We shall now study the behavior of the curves near times in the discrete set $C_{h . P}$ at which solitons collide with antisolitons. To this end it is expedient to introduce the 'multiple collision' and 'smooth' sets

$$
C_{h, P}^{m} \equiv\left\{t \in \boldsymbol{R} \mid \sigma\left(A_{h}(t, P)\right) \text { is not simple }\right\}, \quad S_{h, P} \equiv \boldsymbol{R} \backslash C_{h, P}^{m}
$$

Thus, the former set consists of times for which at least two solitons or two antisolitons collide. Of course, this can only happen when an $\bar{s}$-collision 
occurs at the same time and position, so that $C_{h, P}^{m} \subset C_{h, P}$. Before showing by example that $C_{h, P}^{m}$ need not be empty, we summarize and extend the above findings.

Theorem 6.7. Let $\tau=\pi / 2, h \in \mathscr{C}$ and $P \in \Omega^{b}$. For any $t \in S_{h, P}$ the matrix $A_{h}(t, P)$ has distinct eigenvalues, of which $N_{+}\left(N_{-}\right)$are positive (negative). Ordering the eigenvalues according to (6.8), the position part of the piecewise integral curve $\boldsymbol{R} \backslash C_{h, P} \rightarrow \Omega^{b}, t \mapsto \exp \left(t H_{h}\right)(P)$ defined above is given by (6.9). It has a continuous extension to $\mathbb{R}$ which is real-analytic on $S_{h, P}$.

Now let $T \in C_{h, P}$. Then the matrix elements of $L$ remain bounded as $t \rightarrow T$. Suppose $x_{i}^{+}, x_{j}^{-} \rightarrow x_{0}$ for $t \rightarrow T$, while no other $x_{k}^{\delta}$ converges to $x_{0}$. Then $p_{i}^{+}+p_{j}^{-}$remains bounded as $t \rightarrow T$, whereas either one has

$$
\begin{aligned}
& \lim _{t \rightarrow T} p_{i}^{+}=\infty, \quad \lim _{t \rightarrow T} p_{j}^{-}=-\infty \\
& \lim _{t \rightarrow T} \operatorname{ext}\left(\operatorname{sh} p_{i}^{+} V_{i}^{+}\right) \in(0, \infty), \lim _{t \rightarrow T} \operatorname{ext}\left(-\operatorname{sh} p_{j}^{-} V_{j}^{-}\right) \in(0, \infty), \quad \text { ext }=\inf , \text { sup }
\end{aligned}
$$

or the same formulas hold true with $p_{i}^{+}, p_{j}^{-}$replaced by $-p_{i}^{+},-p_{j}^{-}$.

Proof. The first two assertions have already been proved. The third one easily follows from the discreteness of $C_{h . P}$ and the fact that the spectrum of $A_{h}(t, P)$ is continuous in $t$ on $\boldsymbol{R}$ and real-analytic in $t$ wherever it is simple.

To prove the fourth assertion we recall that any $k^{\text {th }}$ order principal minor $M_{k}$ of $L$ is positive on $\Omega^{b}$. Since the flow is isospectral, it follows that $M_{k}\left(L_{t}\right)$ is bounded above by $S_{k}\left(L_{0}\right)$ and bounded below by 0 for any $t \in \boldsymbol{R} \backslash C_{h, P}$. In particular, this entails that the diagonal elements remain bounded as $t \rightarrow T$. Recalling (2.70) we infer that, for instance,

$$
\begin{aligned}
\left|L_{i, N_{+}+j}\right|^{2} & =\exp \left(\beta\left[p_{i}^{+}+p_{j}^{-}\right]\right) V_{i}^{+} V_{j}^{-} / \operatorname{sh}^{2} \frac{1}{2} \mu\left(x_{i}^{+}-x_{j}^{-}\right) \\
& \leqslant-L_{i, N_{+}+j} L_{N_{+}+j, i}+L_{i i} L_{N_{+}+j, N_{+}+j} \leqslant S_{2}\left(L_{0}\right)
\end{aligned}
$$

and so $L_{t}$ remains bounded as $t \rightarrow T$.

To prove the last assertion we note the assumption entails 


$$
\lim _{t \rightarrow T} V_{i}^{+} V_{j}^{-} / \operatorname{sh}^{2} \frac{1}{2} \mu\left(x_{i}^{+}-x_{j}^{-}\right)=1
$$

cf. (1.3), (1.4). In view of (6.113) this implies that $p_{i}^{+}+p_{j}^{-}$remains bounded above as $t \rightarrow T$. Likewise, consideration of the relevant term in $S_{N}^{-1} S_{N-2}$ leads to the conclusion that $p_{i}^{+}+p_{j}^{-}$remains bounded below.

Next, we develop $\left|L-\lambda \mathbb{1}_{N}\right| \equiv P(\lambda)$ w.r.t. row $i$ and then w.r.t. row $N_{+}+j$. Then we obtain the equality

$$
\begin{aligned}
P(\lambda)= & {\left[\lambda^{2}+\exp \left(\beta\left[p_{i}^{+}+p_{j}^{-}\right]\right) V_{i}^{+} V_{j}^{-} / \operatorname{sh}^{2} \frac{1}{2} \mu\left(x_{i}^{+}-x_{j}^{-}\right)\right] Q(\lambda) } \\
& +\exp \left(\frac{1}{2} \beta p_{i}^{+}\right)\left(V_{i}^{+}\right)^{1 / 2} R(\lambda)+\exp \left(\frac{1}{2} \beta p_{j}^{-}\right)\left(V_{j}^{-}\right)^{1 / 2} S(\lambda)
\end{aligned}
$$

where $Q, R, S$ are polynomials whose coefficients remain bounded as $t \rightarrow T$. Now assume both $p_{i}^{+}$and $p_{j}^{-}$remain bounded. Then the second and third term at the rhs converge to 0 as $t \rightarrow T$, so that two roots of $P(\lambda)$ move towards the imaginary axis. But $P(\lambda)$ does not depend on $t$, a contradiction.

Therefore, as $t \rightarrow T-$, one must have either $p_{i}^{+} \rightarrow \infty, p_{j}^{-} \rightarrow-\infty$ or $p_{i}^{+} \rightarrow-\infty, p_{j}^{-} \rightarrow \infty$. In the first case (e.g.) the third term converges to 0 , so that $\exp \left(\beta p_{i}^{+}\right) V_{i}^{+}$must stay at a finite distance from 0 for $t \rightarrow T-$ to avoid moving eigenvalues. Since $p_{i}^{+}+p_{j}^{-}$and $V_{i}^{+} / V_{j}^{-}$remain bounded, this must hold true for $\exp \left(-\beta p_{j}^{-}\right) V_{j}^{-}$, too. Thus for $t \rightarrow T-$ the desired conclusion follows. Clearly, for $t \rightarrow T+$ we can argue in the same way, so that we are now reduced to showing that $p_{i}^{+}$(say) has equal limits from the right and the left. We assume that this is not the case and derive a contradiction.

To this end we recall that near $t=T$ the integral curve is the pointwise limit of a global integral curve of $H_{h}(g)$ as $g \uparrow \pi / \mu \beta$. From the assumption it readily follows that $p_{i}^{+}(g, t)$ has a zero at $t=t_{g}$ such that $t_{g} \rightarrow T$ for $g \uparrow \pi / \mu \beta$. Considering now the generalization of (6.115) to $g \leqslant \pi / \mu \beta$ we arrive at the desired contradiction: Taking $t=t_{g}$ and sending $g$ to $\pi / \mu \beta$ the second and third term go to 0 , so that two eigenvalues of $L\left(g, t_{g}\right)$ move towards the imaginary axis.

Of course, one has $S_{h, P}=\boldsymbol{R}$ when $N_{+}=N_{-}=1$. Next, we detail the simplest case for which $C_{h, P}^{m}$ is not empty: Take 


$$
\begin{aligned}
& N_{+}=2, \quad N_{-}=1, \quad h(z)=\operatorname{ch} \beta z, \quad x_{1}^{+}=-x_{2}^{+} \equiv x>0, \\
& p_{1}^{+}=-p_{2}^{+} \equiv p<0, \quad x^{-}=p^{-} \equiv 0 .
\end{aligned}
$$

Then it is readily verified that a triple collision occurs at the origin after time $T>0$. More specifically one finds

$$
\begin{aligned}
& x^{-}(t)=p^{-}(t)=0 \\
& t \rightarrow T \pm \Rightarrow \pm \dot{x}_{1}^{+}(t), \pm \dot{x}_{2}^{+}(t), \pm \frac{1}{2} \beta \operatorname{sh} \beta p_{1}^{+}(t), \mp \frac{1}{2} \beta \operatorname{sh} \beta p_{2}^{+}(t) \rightarrow \frac{1}{2} \beta\left(P_{0}^{2}-1\right)^{1 / 2}
\end{aligned}
$$

where

$$
P_{0}=2 \operatorname{ch} \beta p \text { th } \frac{1}{2} \mu x \operatorname{cth} \mu x+\operatorname{th}^{2} \frac{1}{2} \mu x>1 .
$$

Thus the collision amounts to elastic reflection of the two solitons. Note that none of the momenta diverges for $t \rightarrow T$. Note also the initial point belongs to the exceptional set $\Omega_{e}^{b}$ if and only if $P_{0}(x, p)=3$.

For $N \geqslant 4$ we have no information on $C_{h . P}^{m}$ for $P \in \Omega_{e}^{b}$ and $h(z)$ nonlinear. In contrast, for $P \in \Omega_{s}^{b}$ a quite detailed picture can be obtained, but it is convenient to determine first the long-time asymptotics of the positions. To this end we fix $P \in \Omega_{l}^{b}$ and proceed in the same way as for $\tau<\pi / 2$. Thus, (6.83) should again be substituted in (6.16), together with (3.57). Now, we need not and shall not consider the limit $\delta_{n} \downarrow-\mu g$, as this takes us out of $\Omega^{b}$ (recall (5.90)). But (6.85) and (6.30) are still valid, while (6.86)-(6.88) may be replaced by

$$
\begin{aligned}
& \delta_{c}: S_{\mu g} \backslash\{0\} \rightarrow \mathbb{R}, \quad z \mapsto 2 \mu^{-1} \ln \left|\operatorname{cth} \frac{1}{2} \beta z\right|, \quad \beta \mu g=\pi \\
& \delta_{p}: S_{\mu g} \backslash\{0\} \rightarrow 2 \mu^{-1}(-\pi, \pi], \quad z \mapsto 2 \mu^{-1} \arg \left(\operatorname{cth} \frac{1}{2} \beta z\right), \quad \beta \mu g=\pi .
\end{aligned}
$$

Theorem 6.8. Let $\tau=\pi / 2, h \in \mathscr{C}$ and $P \in \Omega_{l}^{b}$. Denote by $x^{+}(t), x^{-}(t), t \in \mathbb{R}$, the continuous extension of the position part of the piecewise integral curve $\exp \left(t H_{h}\right)(P), t \in \mathbb{R} \backslash C_{h, P}$. Then the assertions of Theorem 6.2 hold true with the following changes: $\delta_{c}$ and $\delta_{p}$ are given by (6.119) and (6.120), in (6.46) one should substitute 


$$
\left[\frac{\mu^{2} g^{2}}{\delta_{n}^{2}}-1\right]^{1 / 2} \rightarrow\left|\cot \frac{1}{2} \beta \delta_{n}\right|
$$

and the assertion (6.48) should be omitted.

Proof. Using (6.103) with $t \in \boldsymbol{R} \backslash C_{h . P}$, this follows from (6.49), (6.50) and (6.85) in the same way as before.

Just as in the previous case, the remarks after Th. 6.2 can easily be adapted. Here, (6.51) may be replaced by

$$
\delta(z) \equiv 2 \mu^{-1} \ln \left(\operatorname{cth} \frac{1}{2} \beta z\right), \quad z \neq \pi i k \beta^{-1}, \quad k \in Z, \quad \beta \mu g=\pi
$$

and then $\delta_{c}$ and $\delta_{p}$ are equal to $\operatorname{Re} \delta$ and $\operatorname{Im} \delta$, resp.

As we have seen at the end of Section $5 \mathrm{~B}$, dead breathers decouple altogether in the case at hand. Therefore, no counterpart of Ths. 6.3 and 6.6 exists. However, in the present case three or more positions can become equal at the same time, and we continue by shedding more light on this multiple collision phenomenon. As announced above, we restrict our considerations to $\Omega_{l}^{b}$. A suitable use of (6.6) then enables us to reduce dynamical questions concerning occurrence and character of multiple collisions to a problem that is of a kinematical nature. This problem consists in describing the collision sets

$$
D_{l}^{c} \equiv D_{l} \backslash D_{l}^{b}, \quad l \in\{0,1, \cdots, m\}
$$

and the possible spectra of $\tilde{A}(v, a)$ on $D_{l}^{c}$. Indeed, once this problem is solved, one can use (6.6) to establish which points in

$$
\hat{\Omega}_{l}^{c} \equiv \hat{\Omega}_{l} \backslash \hat{\Omega}_{l}^{b}=\mathscr{C}_{l}\left(D_{l}^{c}\right)
$$

can be reached from $\hat{\Omega}_{l}^{b}$ for the dynamics at hand.

We shall not present a complete solution to this problem. However, we are going to show that $\tilde{A}$ can have multiple eigenvalues on $D_{l}^{c}$ for any $N \geqslant 3$ and $l \in\{0, \cdots, m\}$, but that certain constraints on the spectrum do occur (in addition to (2.57), of course).

First of all, let us note that so far we have not even shown that the set

$$
D^{c} \equiv \bigcup_{l=0}^{m} D_{l}^{c}
$$


is non-empty. But this easily follows from Th. 6.8: choosing $h(z)=\operatorname{ch} \beta z$ (e.g.) and suitable momenta, it follows upon comparing the $t \rightarrow \infty$ and $t \rightarrow-\infty$ asymptotics that soliton/antisoliton collisions must have occurred along the orbit (infinitely often for $l>0$ ).

Fixing now $\hat{P}^{c} \in \hat{\Omega}_{l}^{c}$, we can also use Th. 6.8 to conclude that a suitable choice of $h$ ensures that the orbit of $\hat{P}^{c}$, defined by the rhs of (6.6), will not remain in $\hat{\Omega}_{l}^{c}$. As a consequence there exist $h \in \mathscr{C}, P \in \Omega_{l}^{b}$ and $T>0$ such that $\exp \left(t \hat{H}_{h, l}\right)(\hat{P}) \rightarrow \hat{P}^{c}$ as $t \rightarrow T$.

Next, we recall from Th. 6.7 that $L_{t}$ remains bounded as $t \rightarrow T$. Thus, there exists a sequence $t_{n} \rightarrow T$ such that $L_{t_{n}}$ has a limit $L_{T}$. Now we also have

$$
\lim _{t \rightarrow T} A_{t}=\operatorname{diag}\left(\exp \left[\mu x_{1}^{+}(T)\right], \cdots,-\exp \left[\mu x_{N_{-}}^{-}(T)\right]\right)
$$

Moreover, we may write (cf. (2.69), (2.70))

$$
e_{k}=\left\{\begin{array}{l}
\left(A_{k k} L_{k k}\right)^{1 / 2} \\
i\left(-A_{k k} L_{k k}\right)^{1 / 2}
\end{array} \quad k=\left\{\begin{array}{l}
1, \cdots, N_{+} \\
N_{+}+1, \cdots, N
\end{array}\right.\right.
$$

so it follows that $e_{t_{n}}$ has a limit $e_{T}$. But now it follows from (2.7) that

$$
L_{T} A_{T}+A_{T} L_{T}=2 e_{T} \otimes e_{T}
$$

Here, $L_{T}$ is a $\mathscr{J}$-s.a. matrix with non-negative $(\delta \delta)$-elements and purely imaginary $(\delta,-\delta$ )-elements (recall (2.22)), and with simple spectrum in the open right half plane, $A_{T}$ is a matrix of the form

$$
\operatorname{diag}\left(\alpha_{1}, \cdots, \alpha_{N}\right), \quad \alpha_{1} \geqslant \cdots \geqslant \alpha_{N_{+}}>0, \quad \alpha_{N_{+}+1} \leqslant \cdots \leqslant \alpha_{N}<0
$$

and $e_{T k}$ is given by the $r h s$ of (6.127) with $A, L \rightarrow A_{T}, L_{T}$, these features being preserved under the limit $t_{n} \rightarrow T$, cf. (2.70).

Conversely, assume $L$ is a matrix with the above properties and $A$ is a matrix of the form (6.129); defining $e$ by (6.127), assume in addition that $L$, $A, e$ fulfil (6.128) (with subscripts omitted). Then all assumptions of Section $2 \mathrm{~A}$ are satisfied, so we are again lead to $(2.82)$ with $(v, a) \in D$.

The upshot of this train of thought is, that we obtain a well-defined surjection

$$
\mathscr{D}^{\#}: \mathscr{P}_{s} \rightarrow D, \quad(A, L) \mapsto(v, a)
$$

where $\mathscr{P}_{s}$ denotes the set of all pairs $A, L$ with the above-mentioned 
properties. Later on, we shall detail the relation between $\Omega_{s}^{b}$ and $\mathscr{P}_{s}$, and discuss the extent to which $\mathscr{D}^{\#}$ fails to be injective. Right now, our aim is still to obtain information on the possible spectra of $\tilde{A}$ on $D^{c}$, and the above developments enable us to trade this problem for the more accessible problem of characterizing those $A$ of the form (6.129) for which a pair $(A, L) \in \mathscr{P}_{s}$ exists. (Recall that the above scattering theory argument ensures that whenever a pair $(A, L)$ belongs to $\mathscr{P}_{s}$, one can find $\left(A, L_{T}\right) \in \mathscr{P}_{s}$ with the same image under $\mathscr{D}^{\#}$.)

As a first step along these lines, we note that pairs $(A, L) \in \mathscr{P}_{s}$ exist such that: (1) $\mathscr{D}^{\sharp}(A, L) \in D_{l}^{c}$; (2) $\sigma(A)$ is simple; (3) $k \leqslant l$ pairs of solitons/antisoliton positions coincide. Indeed, using a by now familiar perturbation argument the existence proof can be reduced to the case $N_{+}=N_{-}=1$, where we can take (e.g.)

$$
A=\left[\begin{array}{cc}
a & 0 \\
0 & -a
\end{array}\right], \quad L=\left[\begin{array}{cc}
0 & i b \\
i b & c
\end{array}\right], \quad a, b, c>0, \quad c \neq 2 b .
$$

As a second step, suppose that for $(A, L) \in \mathscr{P}_{s}$ an eigenvalue $\alpha$ of $A$ has multiplicity $n>1$. Then we have $L_{j k}\left(\alpha+\alpha_{k}\right)=2 e_{j} e_{k}$ for the $n$ indices $j$ that are involved. Since $|L| \neq 0$, we deduce $\alpha+\alpha_{k}=0$ for at least $n-1$ indices $k$, and repeating the argument for $-\alpha$ we deduce $\alpha+\alpha_{k}=0$ for at most $n+1$ indices $k$. Therefore, the numbers $c_{+} / c_{-}$of solitons/antisolitons involved in any multiple collision must satisfy $\left|c_{+}-c_{-}\right| \leqslant 1$.

Finally, to show that degeneracies (and hence multiple collisions) do occur, it suffices to prove the claim that for any $N_{+}, N_{-}$such that $N_{+}+N_{-} \geqslant 3$, $\left|N_{+}-N_{-}\right| \leqslant 1$, there exist $(A, L) \in \mathscr{P}_{s}$ such that $|\sigma(A)|=2$. Indeed, using perturbation arguments we may then conclude that in (6.129) one can have arbitrary multiplicities subject to the constraint in the previous paragraph, provided the distances between the positions that are involved are sufficiently large. (Actually we believe that the proviso may be omitted; to prove this an explicit determination of $\mathscr{P}_{s}$ is probably required.)

To prove the claim just made, it would be enough to exhibit pairs $A, L$ with the stated properties. However, we shall proceed differently, so as to obtain an explicit picture of the all-in collision subset of $D^{c}$. In view of (4.19) this amounts to an explicit description of those points $(\hat{q}, \hat{\theta})$ in

$$
\tilde{\hat{\Omega}} \equiv \bigcup_{l=0}^{m} \tilde{\hat{\Omega}}_{l}=\hat{\pi}^{-1}(\hat{\Omega})
$$


for which $\mathscr{L}(\hat{q}, \hat{\theta})$ has spectrum $\{\alpha,-\alpha\}$.

To embark on this we introduce

$$
\hat{\Omega}_{l}^{\text {all }} \equiv\left\{\hat{P} \in \hat{\Omega}_{l} \mid \exists \alpha>0 \quad \text { such that } \quad \sigma\left(\mathscr{L}\left(\hat{\pi}^{-1}(\hat{P})\right)\right)=\{\alpha,-\alpha\}\right\}
$$

and assert that one has

$$
\begin{aligned}
& \operatorname{dim}\left(\hat{\Omega}_{l}^{\text {all }}\right)=N+1, \quad l=0, \cdots, m, \quad\left|N_{+}-N_{-}\right| \leqslant 1, \quad N \geqslant 2 \\
& \bigcup_{l=0}^{m} \hat{\Omega}_{l}^{\text {all }}=\varnothing, \quad\left|N_{+}-N_{-}\right|>1 .
\end{aligned}
$$

More in detail, we assert that for $\left|N_{+}-N_{-}\right| \leqslant 1$ the points in $\hat{\Omega}_{l}^{\text {all }}$ correspond to $(\hat{q}, \hat{\theta}) \in \tilde{\hat{\Omega}}_{l}$ for which $\hat{\theta}_{1}, \cdots, \hat{\theta}_{N}$ are solely constrained by a certain ordering and for which $\operatorname{Im} \hat{q}_{1}, \cdots, \operatorname{Im} \hat{q}_{N}$ are fixed and $\operatorname{Re} \hat{q}_{1}=\cdots=\operatorname{Re} \hat{q}_{N}$. To prove these assertions, we observe that points in $\tilde{\hat{\Omega}}$ yielding spectrum $\{\alpha,-\alpha\}$ can be characterized by equality of $\mathscr{L}^{-1}$ and $\alpha^{-2} \mathscr{L}$ (since $\mathscr{L}$ is diagonalizable on $\tilde{\hat{\Omega}}$ ), and that $\mathscr{L}^{-1}$ can be calculated explicitly. Specifically, we have from (3.57) $-(3.60)$ and (B1)

$$
\mathscr{L}(\hat{q}, \hat{\theta})_{j k}=\frac{\exp \left[-\frac{1}{2} \beta\left(\hat{\theta}_{j}+\hat{\theta}_{k}\right)\right]}{\operatorname{ch} \frac{1}{2} \beta\left(\hat{\theta}_{j}-\hat{\theta}_{k}\right)} \exp \left(\mu \hat{q}_{k}+\beta \hat{\theta}_{k}\right)(-)^{k-1} \prod_{n \neq k} \operatorname{cth} \frac{1}{2} \beta\left(\hat{\theta}_{k}-\hat{\theta}_{n}\right) .
$$

Since the Cauchy matrix is symmetric in the present case, Lemma B2 now leads to the striking relation

$$
\mathscr{L}^{-1}(\hat{q}, \hat{\theta})_{j k}=(-)^{j+k} \exp \left[-\mu\left(\hat{q}_{j}+\hat{q}_{k}\right)\right] \mathscr{L}(\hat{q}, \hat{\theta})_{j k} \quad(\tau=\pi / 2) .
$$

Consequently, the desired spectrum occurs if and only if

$$
\exp \mu\left(\hat{q}_{j}+\hat{q}_{k}\right)=(-)^{j+k} \exp 2 \mu x, \quad x=\mu^{-1} \ln \alpha .
$$

Taking $j=k$, this equality entails

$$
\hat{q}_{j}=x+i s_{j} \pi / \mu(\bmod 2 \pi i / \mu), \quad s_{j} \in\{0,1\}, \quad j=1, \cdots, N
$$

and then it follows that $(6.138)$ is equivalent to $(6.139)$ with $s_{j}$ being equal to 
$0 / 1$ for $j$ odd/even or for $j$ even/odd. Now one infers from (4.23) $-(4.24)$ both choices can be made for the breather $\hat{q}_{j}$ 's. However, from (4.21) and (4.22) one sees that $k_{+}$and $k_{-}$cannot be chosen at will. Indeed, to ensure alternation of 0 and $\pi / \mu$ both in (4.21) and in (4.22) the $s$ and $\bar{s}$ momenta have to alternate in the natural ordering. (If e.g. two soliton momenta occur successively, the product in (4.21) would not change sign.) As a consequence, one cannot have $\mathscr{L}^{-1}=\alpha^{-2} \mathscr{L}$ unless $\left|k_{+}-k_{-}\right|=\left|N_{+}-N_{-}\right| \leqslant 1$. Moreover, the latter constraint suffices: For $\left|N_{+}-N_{-}\right| \leqslant 1$ it is possible to interlace the $s$ and $\bar{s}$ momenta in the right way; taking then $\operatorname{Re} \hat{q}_{j}=x$, alternation of zeros and ones in (6.139) results for $\hat{q}_{1}, \cdots, \hat{q}_{k_{+}}$and for $\hat{q}_{k_{+}+2 l+1}, \cdots, \hat{q}_{N}$; choosing finally the corresponding alternation for $\hat{q}_{k_{+}+1}, \cdots, \hat{q}_{k_{+}+l}$ ensures (6.138). Therefore, the proof of the above assertions is now complete.

To finish this section, we tie up some of the loose ends (creating some new ones in the process). First, we tie up the ends of the piecewise integral curves. Consider the set of triples $(P, h, t), P \in \Omega^{b}, h \in \mathscr{C}, t \in \boldsymbol{R}$, with the equivalence relation

$$
\begin{aligned}
& \left(P_{1}, h_{1}, t_{1}\right) \sim\left(P_{2}, h_{2}, t_{2}\right) \Leftrightarrow\left(t_{1},-t_{2}\right) \in \mathbb{R}^{2} \backslash C_{h_{1}, h_{2}, P_{1}} \quad \text { and } \\
& \exp \left(-t_{2} H_{h_{2}}+t_{1} H_{h_{1}}\right)\left(P_{1}\right)=P_{2} .
\end{aligned}
$$

We denote the set of equivalence classes by $\Omega^{\sharp}$, and inject $\Omega^{b}$ into $\Omega^{\sharp}$ by setting $i(P) \equiv(P, 0,0)$. Now fix $h \in \mathscr{C}$. We are going to define a global transformation group $\exp \left(t H_{h}^{\sharp}\right)$ on $\Omega^{\#}$ that coincides with (the image under $i$ of) $\exp \left(t H_{h}\right)$ on (the image of) $\Omega^{b}$.

To this end we fix a triple $\left(P_{1}, h_{1}, t_{1}\right)$. For $t \notin C_{h . P_{1}}$ we put $\exp \left(t \tilde{H}_{h}\right)\left(P_{1}, h_{1}, t_{1}\right)$ $\equiv\left(\exp \left(t H_{h}\right)\left(P_{1}\right), h_{1}, t_{1}\right)$. Fixing now $T \in C_{h, P_{1}}$, one can find $\epsilon>0$ such that $-\epsilon, T-\epsilon \notin C_{h, P_{1}}$. Clearly, the triple $\left(P_{2}, h_{2}, t_{2}\right) \equiv\left(\exp \left(-\epsilon H_{h}\right)\left(P_{1}\right), \epsilon h+t_{1} h_{1}, 1\right)$ is equivalent to $\left(P_{1}, h_{1}, t_{1}\right)$ and one has $T \notin C_{h_{2}, P_{2}}$. Thus we may define $\exp \left(T H_{h}\right)\left(P_{2}, h_{2}, t_{2}\right) \equiv\left(\exp \left(T H_{h}\right)\left(P_{2}\right), h_{2}, t_{2}\right)$.

Now assume $\left(P_{3}, h_{3}, t_{3}\right) \sim\left(P_{1}, h_{1}, t_{1}\right)$. Then one easily checks $\left(\exp \left(t H_{h}\right)\left(P_{1}\right), h_{1}, t_{1}\right)$ $\sim\left(\exp \left(t H_{h}\right)\left(P_{3}\right), h_{3}, t_{3}\right)$ for $t \notin C_{h, P_{1}} \cup C_{h, P_{3}}$. Consequently, fixing an equivalence class $Q$ of triples and fixing $t \in \boldsymbol{R}$, there exists a non-empty subclass of triples (in general depending on $t$ ) on which $\exp \left(t \tilde{H}_{h}\right)$ is defined, yielding triples that belong to an equivalence class $Q_{t}$.

The upshot is, that the partially defined map $\exp \left(t \tilde{H}_{h}\right)$ on triples descends to an everywhere defined map $\exp \left(t H_{h}^{\sharp}\right)$ on $\Omega^{\sharp}$, and there is no difficulty in verifying that this gives rise to a 1-parameter group with the properties 
announced above. As a result, we have now completed the piecewise flows on $\Omega^{b}$ to global flows on the 'phase space' $\Omega^{\#}$. In an obvious sense, $\Omega^{\sharp}$ is the minimal extension of $\Omega^{b}$ serving this purpose.

However, the above construction has the advantage of theft over toil: One really wants a concrete picture of $\Omega^{\sharp}$, preferably as a manifold in which $\Omega^{b}$ is dense, equipped with a symplectic structure that coincides with $\omega$ on $\Omega^{b}$.

To supply at least part of such a picture, consider first the extension $\Omega_{l}^{\sharp}$ of $\Omega_{l}^{b}$. The map sending the triple $(P, h, t)$ to the point $\exp \left(t \hat{H}_{h, l}\right)(\hat{P})$ in $\hat{\Omega}_{l}$ (cf.(6.6)) clearly descends to $\Omega_{l}^{\#}$, and then given rise to a bijection between $\Omega_{l}^{\#}$ and $\hat{\Omega}_{l}$ that intertwines the flows. Thus $\Omega_{l}^{\sharp}$ may be viewed as the manifold $\hat{\Omega}_{l}$ (in which $\hat{\Omega}_{l}^{b}$ is indeed dense), and the extension $\Omega_{s}^{\sharp}$ of $\Omega_{s}^{b}$ may be thought of as $\hat{\Omega}$.

To get a more explicit picture of all of $\Omega^{\#}$, we introduce a set $\mathscr{P}$ of pairs $(A, L)$ which is defined just as the above pair set $\mathscr{P}_{s}$, except that $\sigma(L)$ need not be simple. Any point $P=(x, p) \in \Omega^{b}$ gives rise to a pair $(A, L) \equiv(A(x), L(x, p)) \in \mathscr{P}$ for which $L$ has positive diagonal elements, cf. (2.70). Conversely, suppose $(A, L) \in \mathscr{P}$ and the diagonal elements of $L$ are positive. From $L_{i j}\left(\alpha_{i}+\alpha_{j}\right)=2 e_{i} e_{j}$ with $i=j$ we then infer $e_{1}, \cdots, e_{N} \neq 0$; taking $i \neq j$ now yields $\alpha_{i}+\alpha_{j} \neq 0$; since $|L| \neq 0$, one must have strict inequalities in (6.129); thus, a unique $P=(x, p) \in \Omega^{b}$ exists satisfying $(A(x), L(x, p))=(A, L)$.

To summarize: We may view $\Omega_{(s)}^{b}$ as a subset of $\mathscr{P}_{(s)}$ and for any $(A, L)$ in

$$
\mathscr{P}_{(s)}^{c} \equiv \mathscr{P}_{(s)} \backslash \Omega_{(s)}^{b}
$$

at least one diagonal element of $L$ vanishes; moreover, for $(A, L) \simeq P \in \Omega_{s}^{b}$ one has $\mathscr{D}^{\sharp}(A, L)=\mathscr{D}(P)$, so $\mathscr{D}^{\sharp}$ may viewed as an extension of $\mathscr{D}$.

The point is now, that there exists a quite natural correspondence between $Q \in \Omega^{\sharp}$ and a non-empty subset $\Lambda(Q)$ of $\mathscr{P}$. To detail this, let $(P, h, T) \in Q$. Then this triple yields at least one pair $(A, L) \in \mathscr{P}$, where $L$ is the limit of a sequence $L_{t_{n}}$ as considered above. Letting the sequences and the representants of $Q$ vary, one gets of course the same $A$, but if $Q \in \Omega^{\sharp} \backslash \Omega^{b}$, then $L$ can be highly non-unique. For instance, one readily verifies that the triple collision point in $D^{c} \simeq \Omega_{s}^{\#} \backslash \Omega_{s}^{b}$ arising from (6.116) for $t \rightarrow T$ yields a 1-parameter family of $L$ (which reflects the fact that $A$ has a non-trivial commutant).

We now define $\Lambda(Q)$ as the set of all pairs $(A, L)$ obtained from triples in $Q$ via limits. Of course, one has $|\Lambda(Q)|=1$ for $Q \in \Omega^{b} \subset \Omega^{\sharp}$. Also, assuming $Q_{1} \neq Q_{2}$, one gets disjoint limit sets $\Lambda\left(Q_{1}\right)$ and $\Lambda\left(Q_{2}\right)$ whenever at least one of $Q_{1}, Q_{2}$ belongs to $\Omega^{b}$. Moreover, for $Q \in \Omega_{s}^{\#}$ corresponding to $\hat{P} \in \hat{\Omega}$ we clearly have 


$$
\Lambda(Q) \subset \mathscr{D}^{\sharp-1}\left(\mathscr{C}^{-1}(\hat{P})\right) .
$$

Thus, $\Lambda\left(Q_{1}\right) \cap \Lambda\left(Q_{2}\right)=\varnothing$ whenever at least one of $Q_{1}, Q_{2}$ belongs to $\Omega_{s}^{\#}$.

Unfortunately, we have no complete proof for the obvious conjecture that $\Lambda\left(Q_{1}\right)$ and $\Lambda\left(Q_{2}\right)$ are disjoint whenever $Q_{1} \neq Q_{2}$. But the proof can be reduced to showing that the snag encountered above is a phantom. More precisely, the conjecture holds true when one can prove that for $h(z)$ nonlinear, there do not exist any piecewise integral curves in $\Omega_{e}^{b}$ with equal $\sigma(L)$ and position parts, but with unequal momentum parts.

To explain this, suppose the triples $\left(P_{i}, h_{i}, T_{i}\right) \in Q_{i} \in \Omega^{\sharp} \backslash \Omega^{b}$ yield the same pair $(A, L)$ via sequences $t_{n i} \rightarrow T_{i}, i=1,2$. Then we have

$$
\begin{aligned}
A\left(\exp \left(t_{2} H_{h_{2}}\right)\left(P_{2}\right)\right) & \sim A_{h_{2}}\left(t_{2}-t_{n 2}, \exp \left(t_{n 2} H_{h_{2}}\right)\left(P_{2}\right)\right) \\
& \sim A \exp \left[\left(t_{2}-T_{2}\right) \mu h_{2}^{\prime}\left(\beta^{-1} \ln L\right)\right] \\
& \sim A_{h_{1}, h_{2}}\left(\left(T_{1}-t_{n 1}, t_{2}-T_{2}\right), \exp \left(t_{n 1} H_{h_{1}}\right)\left(P_{1}\right)\right) \\
& \sim A\left(\exp \left(t_{2} H_{h_{2}}\right)\left(\tilde{P}_{2}\right)\right), \quad \tilde{P}_{2} \equiv \exp \left(-T_{2} H_{h_{2}}+T_{1} H_{h_{1}}\right)\left(P_{1}\right)
\end{aligned}
$$

where the second and third similarities follow by taking $n \rightarrow \infty$. (Note $A\left(\exp \left(t H_{h}\right)(P)\right)$ is well defined even if $t \in C_{h . P}$, cf. Th. 6.7.) Taking now $t_{2}=0$ in (6.143), one infers $\left(T_{1},-T_{2}\right) \in \boldsymbol{R}^{2} \backslash C_{h_{1}, h_{2}, P_{1}}$ and $\left(P_{1}, h_{1}, T_{1}\right) \sim\left(P_{2}, h_{2}, T_{2}\right)$, cf. (6.140)). More generally, (6.143) says that the piecewise integral curve $\exp \left(t_{2} H_{h_{2}}\right)\left(P_{2}\right)$ has the same position part as $\exp \left(t_{2} H_{h_{2}}\right)\left(\tilde{P}_{2}\right)$. Obviously, the spectra of the Lax matrix on these two curves are equal, too. Thus, if one can show all this entails $\tilde{P}_{2}=P_{2}$, then one has $\left(P_{1}, h_{1}, T_{1}\right) \sim\left(P_{2}, h_{2}, T_{2}\right)$ and the conjecture would be proved.

Apart from this open question, we do not know the answer to another obvious question: Can one obtain any $(A, L) \in \mathscr{P}$ via some triple $(P, h, T)$ ? (We have not even answered this question for $\mathscr{P}_{s}^{c}$, which is why we write $\subset$, not $=$, in (6.142).) We conjecture that the answer is 'yes'.

At any rate, the conclusion reads: Provided the two conjectures involved hold true, one may view $\Omega^{\sharp}$ as $\mathscr{P} / \sim$, where $\left(A_{1}, L_{1}\right) \sim\left(A_{2}, L_{2}\right)$ if and only if the pairs belong to the same limit set. 


\section{The Relation to Soliton Solutions Revisited}

The context and the results of this last chapter have been delineated in the Introduction. As announced there, we elaborate on the relation of the particle systems at issue to various soliton PDEs and lattices. We shall restrict ourselves to an explicit consideration of the particle-like solutions to the sine-Gordon, modified $\mathrm{KdV}$ and $\mathrm{KdV}$ equations, in the form

$$
\begin{array}{ll}
\varphi_{y y}-\varphi_{t t}-\sin \varphi=0 & (\mathrm{sG}) \\
v_{t}+24 v^{2} v_{y}+v_{y y y}=0 & (\mathrm{mKdV}) \\
u_{t}-6 u u_{y}+u_{y y y}=0 & (\mathrm{KdV})
\end{array}
$$

However, equations in the associated hierarchies can be handled in similar ways, just as several other soliton hierarchies related to the above particle systems.

In fact, much of the analysis needed to handle the pure soliton case (Section 7A) applies uniformly to the $\mathbb{I I}_{\mathrm{nr}}$ and $\mathbb{I}_{\mathrm{rel}}$ systems. (As will be briefly recalled below, the soliton $\mathrm{PDEs}(7.1)-(7.3)$ all correspond to the $\tau=\pi / 2 \mathbb{I I}_{\mathrm{rel}}$ systems [6].) The generality thus obtained has notational advantages and it renders the results applicable to soliton equations associated with $\tau$-values not equal to $\pi / 2$ (such as the Boussinesq and Hirota-Satsuma equations [2]).

On the other hand, we specialize to the $s G$ and $m K d V$ equations in Section $7 \mathrm{~B}$, where the case of solitons, antisolitons and their bound states is considered. This is because in that case a general setup would be notationally unwieldy; moreover, a study of space-time trajectories would be forbidding without specializing to concrete dynamics.

\section{A. The Pure Soliton Case}

For several reasons it is convenient to trade the symbols $x^{+}, p^{+}, x^{s}, p^{s}$ of the pure soliton case for the symbols $q, \theta, \hat{q}, \hat{\theta}$, resp., cf. the diagram (3.28). (For one, this facilitates comparison with previous work $[1,2,3,6]$.) The connection to soliton solutions can be made via the matrix

$$
\mathscr{L}(\hat{q}, \hat{\theta})_{j k} \equiv C(\beta, 1,-g ; \hat{\theta}, \hat{\theta})_{j k} \eta_{k}(\hat{q}, \hat{\theta}), \quad(\hat{q}, \hat{\theta}) \in \mathbb{R}^{N} \times G_{N}
$$

where $C$ is the Cauchy matrix (B1) and 


$$
\eta_{k} \equiv \exp \left(\hat{q}_{k}+\beta \hat{\theta}_{k}\right) \prod_{j \neq k}\left[1+\sin ^{2}\left(\frac{1}{2} \beta g\right) / \operatorname{sh} \frac{1}{2} \beta\left(\hat{\theta}_{k}-\hat{\theta}_{j}\right)\right]^{1 / 2}
$$

cf. (3.1) $-(3.5)$ and (3.57) $-(3.60)$.

To be specific, pure soliton solutions to various PDEs and lattices can be expressed in terms of the matrix

$$
\tilde{A}(t, y) \equiv \mathscr{L}\left(\hat{q}_{1}+\sigma_{1}\left(v_{1} t-y\right), \cdots, \hat{q}_{N}+\sigma_{N}\left(v_{N} t-y\right), \hat{\theta}\right)
$$

where the parameters depend on the soliton equation at hand. It so happens that in all cases studied thus far the parameters can be chosen to satisfy

$$
\sigma_{1}, \cdots, \sigma_{N}>0, \quad v_{N}<\cdots<v_{1}, \quad \tau=\beta g / 2 \in[0, \pi / 2]
$$

(up to overall signs). In particular, for the $s G$ and $(m) K d V$ cases we may (and shall) take $\beta=1, g=\pi$, and

$$
\begin{aligned}
& \sigma_{j}=\operatorname{ch} \hat{\theta}_{j}, \quad v_{j}=\operatorname{th} \hat{\theta}_{j}, \quad j=1, \cdots, N \quad(\mathrm{sG}) \\
& \sigma_{j}=\exp \hat{\theta}_{j}, \quad v_{j}=\exp 2 \hat{\theta}_{j}, \quad j=1, \cdots, N \quad((\mathrm{~m}) \mathrm{KdV}) .
\end{aligned}
$$

Then the $N$-soliton solutions read [6]

$$
\begin{array}{rlrl}
\varphi & =4 \operatorname{Tr} \operatorname{Arctg} \tilde{A}(t, y) & & (\mathrm{sG}) \\
v & =\partial_{y} \operatorname{Tr} \operatorname{Arctg} \tilde{A}(t, y) & & (\mathrm{mKdV}) \\
u=-2 \partial_{y}^{2} \operatorname{Tr} \ln \left(\mathbb{1}_{N}+\tilde{A}(t, y)\right) & & (\mathrm{KdV}) .
\end{array}
$$

(It should be mentioned that (7.10) and (7.11) are not universally called $N$-soliton solutions. For instance, [11] and [12] would refer to (7.10) as $N$-antikink and $\mathrm{N}$-antisoliton solutions, resp.)

Substituting (7.8) or (7.9) in (7.6) and using the canonical transformation $\mathscr{E}$, one readily deduces

$$
\begin{aligned}
& \operatorname{Tr} \operatorname{Arctg} \tilde{A}(t, y)=\sum_{j=1}^{N} \operatorname{Arctg}\left(\exp \left[q_{j}(t, y)\right]\right) \\
& \operatorname{Tr} \ln \left(\mathbf{1}_{N}+\tilde{A}(t, y)\right)=\sum_{j=1}^{N} \ln \left(1+\exp \left[q_{j}(t, y)\right]\right)
\end{aligned}
$$

where 


$$
q_{j}(t, y)=\left[\exp \left(t H^{0}-y H^{1}\right) \circ \mathscr{E}(\hat{q}, \hat{\theta})\right]_{j}, \quad j=1, \cdots, N
$$

with the space-time translation generators being given by

$$
\begin{aligned}
& H^{0}=\frac{1}{2} \operatorname{Tr}\left(L+L^{-1}\right), \quad H^{1}=\frac{1}{2} \operatorname{Tr}\left(L-L^{-1}\right) \\
& H^{0}=\frac{1}{3} \operatorname{Tr} L^{3}, \quad H^{1}=\operatorname{Tr} L \quad((\mathrm{~m}) \mathrm{KdV}) .
\end{aligned}
$$

(Cf. (6.5), (6.6), specialized to the case $N_{-}=0$.)

As a consequence of these formulas the study of the space-time dependence of soliton solutions can be reduced to the study of the eigenvalues $\exp \left[q_{j}(t, y)\right]$, whose space-time dependence is governed by the particle Hamiltonians (7.16), (7.17). It should be noted at this point that the above representations of soliton solutions in terms of linear superpositions of $N$ single soliton eigenvalue functions differs from similar representations in terms of eigenfunctions of the associated linear problem. (The latter have e.g. been used in $[9,10]$ to prove that the lhs of $(1.14)_{ \pm}$goes to 0 for $t \rightarrow \pm \infty$; cf. also [16] and references given there.) This is because the latter functions are meromorphic in $t$ and $y$, in contrast to the former. Moreover, the latter representation suggests a picture of individual solitons regaining their velocities after interaction, whereas in our picture soliton velocities are exchanged (as is obvious from the following).

We proceed to study the eigenvalues $\exp \left[q_{j}(t, y)\right]$ of $\tilde{A}(t, y)$, without committing ourselves to a special choice of parameters; the only assumption we make is that (7.7) holds true. Once a choice is made, one can always define functions $H^{0}, H^{1}$ such that the (logarithms of the) eigenvalues are given by (7.15). However, this is not necessary for the results we shall obtain at first, as these only involve (7.7) and Appendix C.

We begin by supplementing the definition of space-time trajectories given in [6] with a specification of their asymptotics for $t \rightarrow \pm \infty$. In concrete applications to soliton PDEs this result says that for all practical purposes these particle trajectories coincide with the soliton trajectories, once the latter can be read off from a plot of the solution, i.e., before and after all collisions.

Theorem 7.1. Assume (7.7) holds true and let $\exp \left[q_{N}(t, y)\right]<\cdots<\exp \left[q_{1}(t, y)\right]$ be the eigenvalues of $\tilde{A}(t, y)$. Then the equations

$$
q_{j}(t, y)=0, \quad j=1, \cdots, N, \quad t \text { fixed }
$$


admit uniquely determined solutions $y_{N}(t)<\cdots<y_{1}(t)$. These solutions ('soliton space-time trajectories') obey the estimates

$$
y_{N_{N-j+1}}(t)=\frac{1}{\sigma_{j}}\left(\hat{q}_{j} \mp \frac{1}{2} \Delta_{j}(\hat{\theta})\right)+v_{j} t+O\left(\exp \left(\mp t r_{j}\right)\right), \quad t \rightarrow \pm \infty
$$

where

$$
\begin{aligned}
& \Delta_{j}(\hat{\theta}) \equiv\left[\sum_{k<j}-\sum_{k>j}\right] \ln \left[1+\frac{\sin ^{2} \frac{1}{2} \beta g}{\operatorname{sh}^{2} \frac{1}{2} \beta\left(\hat{\theta}_{j}-\hat{\theta}_{k}\right)}\right] \\
& r_{j} \equiv \min _{k \neq j} \sigma_{k}\left|v_{k}-v_{j}\right| .
\end{aligned}
$$

Proof. We assert that (7.7) entails

$$
\partial_{y} q_{j}(t, y) \in\left[-\sigma_{+},-\sigma_{-}\right] \text {. }
$$

where

$$
\sigma_{ \pm} \equiv \max _{\min }\left(\sigma_{1}, \cdots, \sigma_{N}\right) \in(0, \infty)
$$

This assertion will be justified shortly. Accepting it for the moment, existence and uniqueness of the solutions $y_{j}(t)$ to (7.18) is an obvious consequence.

Next, we prove $(7.19)_{+}$. To this end we note

$$
q_{j}\left(t, \zeta_{j}+v_{j} t\right)=\int_{y_{j}(t)}^{\zeta_{j}+v_{j} t}\left(\partial_{y} q_{j}\right)(t, s) d s, \quad \zeta_{j} \equiv \frac{1}{\sigma_{j}}\left(\hat{q}_{j}-\frac{1}{2} \Delta_{j}(\hat{\theta})\right)
$$

and then use (7.22) to deduce

$$
\left|y_{j}(t)-\zeta_{j}-v_{j} t\right| \leqslant \frac{1}{\sigma_{-}}\left|q_{j}\left(t, \zeta_{j}+v_{j} t\right)\right| .
$$

To estimate the $r h s$ we first recall $\exp \left[q_{j}\left(t, \zeta_{j}+v_{j} t\right)\right]$ is an eigenvalue of 


$$
\tilde{A}\left(t, \zeta_{j}+v_{j} t\right)=\mathscr{L}\left(\hat{q}_{1}+\sigma_{1}\left[\left(v_{1}-v_{j}\right) t-\zeta_{j}\right], \cdots, \frac{1}{2} \Delta_{j}(\hat{\theta}), \cdots, \hat{q}_{N}+\sigma_{N}\left[\left(v_{N}-v_{j}\right) t-\zeta_{j}\right], \hat{\theta}\right)
$$

cf. (7.6). Now let $\sigma_{+} \in S_{j-1}$ and $\sigma_{-} \in S_{N-j}$ be permutations that put the positive numbers $\sigma_{1}\left(v_{1}-v_{j}\right), \cdots, \sigma_{j-1}\left(v_{j-1}-v_{j}\right)$ and the negative numbers $\sigma_{j+1}\left(v_{j+1}-v_{j}\right), \cdots, \sigma_{N}\left(v_{N}-v_{j}\right)$ in the natural order, resp. (Note these numbers are not necessarily distinct.) Then the matrix

$$
E(t) \equiv \Pi_{\sigma} A_{s}\left(0, \zeta_{j}\right) \operatorname{diag}\left(\exp \left[\sigma_{1}\left(v_{1}-v_{j}\right) t\right], \cdots, \exp \left[\sigma_{N}\left(v_{N}-v_{j}\right) t\right]\right) \Pi_{\sigma}^{-1} \sim \tilde{A}\left(t, \zeta_{j}+v_{j} t\right)
$$

where

$$
\Pi_{\sigma} \equiv \operatorname{diag}\left(\Pi_{\sigma_{+}}, 1, \Pi_{\sigma_{-}}\right)
$$

satisfies the assumptions of Appendix C. Here, $A_{s}$ denotes the positive matrix given by (2.54), (2.53) and (2.50). (Note $k_{+}=N$ and $v_{i}=\eta_{i}>0$ in the case at hand.) More specifically, one has

$$
\begin{aligned}
& \mathscr{J}_{\rho}(t)=\mathbb{1}_{\left|I_{\rho}\right|}, \quad \rho=1, \cdots, K \\
& M=\Pi_{\sigma} A_{s}\left(0, \zeta_{j}\right) \Pi_{\sigma}^{-1}
\end{aligned}
$$

and there exists an index $\rho$ such that $d_{\rho}=0,\left|I_{\rho}\right|=1$. Combining (C15) with the cluster matrix calculations in Chapter 6 we obtain

$$
\exp \left[q_{j}\left(t, \zeta_{j}+v_{j} t\right)\right]-1=O\left(\exp \left(-t r_{j}\right)\right), \quad t \rightarrow \infty
$$

and on account of (7.25) we may now deduce (7.19) $)_{+}$The proof of (7.19) is similar.

It remains to prove our assertion (7.22). To this end, suppose $B \in M_{N}(C)$ is positive and has simple spectrum. Now consider

$$
B(\epsilon) \equiv e^{\epsilon D} B e^{\epsilon D}, \quad D \equiv-\frac{1}{2} \operatorname{diag}\left(\sigma_{1}, \cdots, \sigma_{N}\right), \quad \epsilon \in \boldsymbol{R} .
$$

For $|\epsilon|$ small $B(\epsilon)$ has positive simple spectrum $\left\{\gamma_{1}(\epsilon), \cdots, \gamma_{N}(\epsilon)\right\}$ and there exists a smooth orthonormal base $\left\{b_{1}(\epsilon), \cdots, b_{N}(\epsilon)\right\}$ such that $B(\epsilon) b_{j}(\epsilon)=\gamma_{j}(\epsilon) b_{j}(\epsilon)$. Differentiating the identity $\gamma_{j}(\epsilon)=\left(b_{j}(\epsilon), B(\epsilon) b_{j}(\epsilon)\right)$ now yields $\gamma_{j}^{\prime}(\epsilon)=\left(b_{j}(\epsilon), B_{j}^{\prime}(\epsilon) b_{j}(\epsilon)\right)$, since $B(\epsilon)$ is self-adjoint and $b_{j}(\epsilon)$ is a unit vector. Using (7.32) this entails 


$$
\gamma_{j}^{\prime}(0) / \gamma_{j}(0)=2\left(b_{j}(0), D b_{j}(0)\right) \in\left[-\sigma_{+},-\sigma_{-}\right]
$$

Applying this result to $B \equiv A_{s}(t, y),(7.22)$ follows at once.

Next, we determine the long-time asymptotics of the eigenvalue $\exp \left[q_{j}(t, y)\right]$ for arbitrary $y$. (Observe (7.31) specifies the asymptotics along the $j^{\text {th }}$ soliton ray.) The result we are heading for (Theorem D2) can be used in applications to soliton PDEs to obtain quite precise information on the temporal asymptotics of pure soliton solutions, as will be illustrated for the $\mathrm{sG}$ and $(\mathrm{m}) \mathrm{KdV}$ equations. To ease the notation we only consider the $t \rightarrow \infty$ behavior.

We now embark on a preview of Th. 7.2 and its proof; this serves to introduce notation and should be of help in keeping track of the overall strategy. To determine the desired eigenvalue asymptotics, we shall exploit Appendix $\mathrm{C}$ just as in the proof of Th. 7.1. Thus, fixing $j \in\{1, \cdots, N\}$ we are going to consider a matrix

$$
\begin{array}{r}
E(t) \equiv \Pi_{\sigma} A_{s}\left(0, \zeta_{j}\right) \operatorname{diag}\left(\exp \left[\sigma_{1}\left(v_{1}-v_{j}-s\right) t\right], \cdots, \exp \left[\sigma_{N}\left(v_{N}-v_{j}-s\right) t\right]\right) \Pi_{\sigma}^{-1} \sim \tilde{A}\left(t, y_{j}(s, t)\right) \\
y_{j}(s, t) \equiv \zeta_{j}+v_{j} t+s t, \quad s \in \boldsymbol{R}, \quad t>0
\end{array}
$$

which reduces to (7.27) for $s=0$. The permutation $\sigma$ takes care of the desired ordering of the numbers

$$
d_{j i}(s) \equiv \sigma_{i}\left(v_{i}-v_{j}-s\right)
$$

and (7.29), (7.30) apply again. However, the cluster number $K$, cluster sizes $\left|I_{I}\right|, \cdots,\left|I_{K}\right|$ and permutation matrix $\Pi_{\sigma}$ now depend not only on $j$, but also on $s$, in general.

To control the $s$-dependence it is expedient to introduce the quantities

$$
\begin{aligned}
& F_{j i}(s) \equiv \operatorname{sgn}(j-i)\left[d_{j i}(s)-d_{j j}(s)\right], \quad i \neq j \\
& F_{j i}^{ \pm}(s) \equiv F_{j i}(s) \pm \sigma_{j} s \\
& r_{j}(s) \equiv \min _{i \neq j} F_{j i}(s) \\
& r_{j}^{ \pm}(s) \equiv r_{j}(s) \pm \sigma_{j} s .
\end{aligned}
$$

In view of our standing assumption (7.7) we have 


$$
F_{j i}(0) \geqslant r_{j}(0)=r_{j}
$$

(recall (7.21)). Now observe that when $s$ moves away from 0 , some $F_{j i}(s)$ will decrease to 0 , in general. Thus, $r_{j}(s)$ will go to 0 and, consequently, we are no longer entitled to invoke the estimate (C15) (with $d_{\rho}$ equal to $d_{j j}(s)$ $=-\sigma_{j} s$ and $\left.c_{\rho}^{+}(t)=1\right)$. Specifically, this bound can only be used for $t>T_{j}(s) \in(0, \infty)$, where $T_{j}(s)$ is defined by the requirement

$$
\exp \left[-T_{j}(s) r_{j}(s)\right]=C_{-, j} / 3 C_{+, j}
$$

cf. (C13). (Here, $C_{+, j} / C_{-, j}$ denotes the maximal/minimal eigenvalue of $\tilde{A}\left(0, \zeta_{j}\right)$, cf. (7.30).) Put differently, we can only use (C15) for $y$ varying over an interval

$$
\Sigma_{j} \equiv\left[y_{j}\left(-s_{j}, t\right), y_{j}\left(s_{j}, t\right)\right]
$$

around the $j^{\text {th }}$ soliton, where $s_{j}$ is yet to be specified.

Fortunately, for $y$ to the right/left of $\Sigma_{j}$ one only needs upper bounds on $\exp \left(q_{j}\right) / \exp \left(-q_{j}\right)$, resp. As it happens, these can be obtained by combining the monotonicity of $q_{j}(t, y)$ in $y$ already proved above (recall (7.22)) with an estimate on the difference of $\exp \left[q_{j}(t, y)\right]$ and the asymptotic (for $t \rightarrow \infty$ ) single soliton function

$$
\exp \left[q_{j}^{\mathrm{as}}(t, y)\right] \equiv \exp \left[\sigma_{j}\left(\zeta_{j}+v_{j} t-y\right)\right], \quad \zeta_{j} \equiv \frac{1}{\sigma_{j}}\left(\hat{q}_{j}-\frac{1}{2} \Delta_{j}(\hat{\theta})\right)
$$

obtained via (C15) (taking $y$ equal to $y_{j}\left(s_{j}, t\right) / y_{j}\left(-s_{j}, t\right)$ ).

Turning now to the details, we set

$$
\begin{aligned}
& R_{j} \equiv \min \left(r_{j}, \sigma_{j}\left(v_{j-1}-v_{j}\right), \sigma_{j}\left(v_{j}-v_{j+1}\right)\right) \\
& \tilde{r}_{j} \equiv \min \left(R_{j}, \min _{i \neq j} \sigma_{i}^{2}\left|v_{i}-v_{j}\right| / \sigma_{j}\right)
\end{aligned}
$$

and introduce $\tilde{T}_{j} \in(0, \infty)$ by requiring

$$
\exp \left(-\tilde{T}_{j} \tilde{r}_{j}\right)=C_{-, j} / 3 C_{+, j}
$$

Finally, we set

$$
s_{j} \equiv R_{j} / \sigma_{j}
$$




$$
y_{j}^{ \pm} \equiv y_{j}\left( \pm s_{j}, t\right)=\zeta_{j}+v_{j} t \pm s_{j} t, \quad y_{j}^{0} \equiv y_{j}(0, t)=\zeta_{j}+v_{j} t .
$$

From now on we use the symbol $C$ to denote positive constants depending only on the initial value $(\hat{q}, \hat{\theta})$ and on the numbers $\sigma_{1}, \cdots, \sigma_{N}$ (such as $C_{+, j}$, $C_{-, j}$ and positive combinations thereof; in applications to soliton PDEs and lattices one has $\left.\sigma_{j}=\sigma\left(\hat{\theta}_{j}\right)\right)$.

Theorem 7.2. One has

$$
\begin{aligned}
& r_{j}(s) \geqslant \tilde{r}_{j}, \quad s \in\left[-s_{j}, s_{j}\right] \\
& r_{j}^{+}(s) \geqslant R_{j}, \quad s \in\left[0, s_{j}\right] \\
& r_{j}^{-}(s) \geqslant R_{j}, \quad s \in\left[-s_{j}, 0\right] .
\end{aligned}
$$

Now let $t>\tilde{T}_{j}$ and set $q_{j}^{(\text {as) }} \equiv q_{j}^{(\text {as) }}\left(t, y_{j}(s, t)\right)$. Then one has for any $s \in\left[-s_{j}, s_{j}\right]$ :

$$
\begin{aligned}
& q_{j}+t \sigma_{j} s \in[-C, C] \\
& q_{j \pm 1}-t d_{j, j \pm 1}(s) \lessgtr \pm C \\
& \left|\exp \left(q_{j}\right)-\exp \left(q_{j}^{(\mathrm{as})}\right)\right|<C \exp \left(-t r_{j}^{+}(s)\right) \\
& \left|\exp \left(-q_{j}\right)-\exp \left(-q_{j}^{(\mathrm{as})}\right)\right|<C \exp \left(-t r_{j}^{-}(s)\right) .
\end{aligned}
$$

Furthermore,

$$
\begin{aligned}
& \exp \left(q_{j}^{\text {(as) }}\right)<C \exp \left(-t R_{j}\right) \quad s>s_{j} \\
& \exp \left(-q_{j}^{\text {(as) }}\right)<C \exp \left(-t R_{j}\right) \quad s<-s_{j} .
\end{aligned}
$$

Proof. To prove (7.50) we should show

$$
F_{j i}(s) \geqslant \tilde{r}_{j}, \quad|s| \leqslant s_{j}, \quad i \neq j
$$

Consider first

$$
F_{j l}(s)=\sigma_{l}\left(v_{l}-v_{j}\right)+s\left(\sigma_{j}-\sigma_{l}\right), \quad l<j .
$$

Fixing $s \in\left[0, s_{j}\right]$ one has $F_{j l}(s) \geqslant r_{j}$, unless $\sigma_{j}<\sigma_{l}$. But then one gets, using $s_{j} \leqslant v_{j-1}-v_{j}$, 


$$
\begin{aligned}
F_{j l}(s) & \geqslant F_{j l}\left(s_{j}\right) \geqslant \sigma_{l}\left(v_{l}-v_{j}\right)+\left(v_{j-1}-v_{j}\right)\left(\sigma_{j}-\sigma_{l}\right) \\
& =\sigma_{l}\left(v_{l}-v_{j-1}\right)+\sigma_{j}\left(v_{j-1}-v_{j}\right) \geqslant \sigma_{j}\left(v_{j-1}-v_{j}\right) \\
& \geqslant R_{j} .
\end{aligned}
$$

Next, taking $s \in\left[-s_{j}, 0\right]$, one has $F_{j l}(s) \geqslant r_{j}$, unless $\sigma_{j}>\sigma_{l}$. Using $s_{j} \leqslant r_{j} / \sigma_{j}$ $\leqslant \sigma_{l}\left(v_{l}-v_{j}\right) / \sigma_{j}$ one then gets

$$
\begin{aligned}
F_{j l}(s) & \geqslant F_{j l}\left(-s_{j}\right) \geqslant \sigma_{l}\left(v_{l}-v_{j}\right)-\sigma_{l}\left(v_{l}-v_{j}\right)\left(\sigma_{j}-\sigma_{l}\right) / \sigma_{j} \\
& =\sigma_{l}^{2}\left(v_{l}-v_{j}\right) / \sigma_{j} \geqslant \tilde{r}_{j} .
\end{aligned}
$$

Therefore, (7.59) holds for $i<j$, and arguing in a similar way for

$$
F_{j k}(s)=\sigma_{k}\left(v_{j}-v_{k}\right)+s\left(\sigma_{k}-\sigma_{j}\right), \quad k>j
$$

we obtain the same conclusion for $i>j$. Hence, (7.50) follows.

To prove (7.51) we should show

$$
F_{j i}^{+}(s) \geqslant R_{j}, \quad s \in\left[0, s_{j}\right], \quad i \neq j .
$$

To this end we note that

$$
F_{j l}^{+}(s)=\sigma_{l}\left(v_{l}-v_{j}\right)+s\left(2 \sigma_{j}-\sigma_{l}\right), \quad l<j
$$

satisfies $F_{j l}^{+}(s) \geqslant r_{j}$, unless $2 \sigma_{j}<\sigma_{l}$. But then

$$
\begin{aligned}
F_{j l}^{+}(s) & \geqslant F_{j l}^{+}\left(s_{j}\right) \geqslant \sigma_{l}\left(v_{l}-v_{j}\right)+\left(v_{j-1}-v_{j}\right)\left(2 \sigma_{j}-\sigma_{l}\right) \\
& =\sigma_{l}\left(v_{l}-v_{j-1}\right)+2 \sigma_{j}\left(v_{j-1}-v_{j}\right) \geqslant 2 \sigma_{j}\left(v_{j-1}-v_{j}\right) \\
& >R_{j},
\end{aligned}
$$

as desired. Next, consider

$$
F_{j k}^{+}(s)=\sigma_{k}\left(v_{j}-v_{k}\right)+s \sigma_{k}, \quad k>j
$$

Since $F_{j k}^{+}(s) \geqslant F_{j k}^{+}(0) \geqslant r_{j}$, we may now conclude that (7.64) holds true, so (7.51) follows.

Similarly, (7.52) is equivalent to

$$
F_{j i}^{-}(s) \geqslant R_{j}, \quad s \in\left[-s_{j}, 0\right], \quad i \neq j
$$

and these inequalities can be verified just as (7.64). 
We proceed with the proof of the time-dependent estimates. In view of (7.47), (7.42) and the bound (7.50) we are entitled to use Th. C1 for $s \in\left[-s_{j}, s_{j}\right]$, the matrix $E(t)$ being given by (7.34).

Specifically, the first assertion implies (7.53) and (7.54), while (C15) entails (7.55). Noting

$$
\left|\exp \left(-q_{j}\right)-\exp \left(-q_{j}^{\text {as }}\right)\right|=\exp \left(-q_{j}-q_{j}^{\text {as }}\right)\left|\exp \left(q_{j}\right)-\exp \left(q_{j}^{\text {as }}\right)\right|
$$

and using (7.53), (7.44) and (7.55) we deduce (7.56) (note $\left.r^{-}(s)=r^{+}(s)-2 \sigma_{j} s\right)$.

Finally, since $q_{j}(t, y)$ is a decreasing function of $y$ we obtain for $y>y_{j}^{+}$ (recall (7.44), (7.48), (7.49))

$$
\begin{aligned}
\exp \left[q_{j}(t, y)\right]<\exp \left[q_{j}\left(t, y_{j}^{+}\right)\right] \\
\quad \leqslant\left|\exp \left[q_{j}\left(t, y_{j}^{+}\right)\right]-\exp \left[q_{j}^{\mathrm{as}}\left(t, y_{j}^{+}\right)\right]\right|+\exp \left(-\sigma_{j} s_{j} t\right) \\
\quad<C \exp \left(-t R_{j}\right),
\end{aligned}
$$

where we used (7.55) and (7.51) in the last step. Analogously, (7.58) follows by using (7.56) and (7.52).

We proceed by using Th. 7.2 to prove the decay bound $(1.14)_{+}$for the pure soliton solutions of the $\mathrm{sG}$ and $(\mathrm{m}) \mathrm{kdV}$ equations. (It will be clear from this how (1.14)_ can be proved.) In all three cases the decay rate $r$ is given by

$$
r \equiv \min \left(r_{1}, \cdots, r_{N}\right) \equiv \min _{i \neq j} \sigma_{i}\left|v_{i}-v_{j}\right|=\min \left(R_{1}, \cdots, R_{N}\right)
$$

cf. (7.45).

Sine-Gordon. Here, $(1.14)_{+}$amounts to (cf. (7.10), (7.13), (7.44))

$$
\sup _{y \in \boldsymbol{R}}\left|\sum_{j=1}^{N}\left[4 \operatorname{Arctg}\left(\exp \left[q_{j}(t, y)\right]\right)-4 \operatorname{Arctg}\left(\exp \left[q_{j}^{\text {as }}(t, y)\right]\right)\right]\right|=O(\exp (-t r))
$$

To prove this bound, consider the $j^{\text {th }}$ difference. For $y \in\left[y_{j}^{0}, y_{j}^{+}\right]$we can use $|\operatorname{Arctg} a-\operatorname{Arctg} b| \leqslant|a-b|$ and the estimates (7.55), (7.51) to get an $O\left(\exp \left(-t R_{j}\right)\right)$ bound. Similarly, for $y \in\left[y_{j}^{-}, y_{j}^{0}\right]$ and $y<y_{j}^{-}$one gets such a bound from (7.56), (7.52) by noting $|\operatorname{Arctg} a-\operatorname{Arctg} b|=\left|\operatorname{Arctg} b^{-1}-\operatorname{Arctg} a^{-1}\right| \leqslant\left|b^{-1}-a^{-1}\right|$. To estimate the tails $y>y_{j}^{+}$and $y<y_{j}^{-}$one need only use $|a-b| \leqslant|a|+|b|$ and $\left|b^{-1}-a^{-1}\right| \leqslant|b|^{-1}+|a|^{-1}$, and invoke (7.57) and (7.58), resp. Hence, (7.72) 
follows.

Modified KdV. Here we should show (cf. (7.11), (7.13))

$$
\partial_{y} \sum_{j=1}^{N}\left[\operatorname{Arctg}\left(\exp \left[q_{j}(t, y)\right]-\operatorname{Arctg}\left(\exp \left[q_{j}^{\mathrm{as}}(t, y)\right]\right)\right]=O(\exp (-t r))\right.
$$

uniformly in $y$. Note first that when the $y$-partial is omitted, then the bound follows just as (7.72). Thus, the new problem consists in proving that the $y$-differentiation does not alter the long-time asymptotics. Specifically, we are going to prove

$$
\sup _{y \in \boldsymbol{R}}\left|\frac{\partial_{y} q_{j}(t, y)}{\operatorname{ch} q_{j}(t, y)}+\frac{\sigma_{j}}{\operatorname{ch} q_{j}^{\mathrm{as}}(t, y)}\right|=O\left(\exp \left(-t R_{j}\right)\right)
$$

from which (7.73) is immediate, cf. (7.71).

First, we observe $1 / \operatorname{ch} q_{j}^{\text {as }}(t, y)$ is $O\left(\exp \left(-t R_{j}\right)\right)$ for $y>y_{j}^{+}$and $y<y_{j}^{-}$due to (7.57) and (7.58), resp. Now note the function $\left(\partial_{y} q_{j}\right)(t, y)$ is bounded on $\mathbb{R}^{2}$. (It equals $-\exp \left[\theta_{j}(t, y)\right] V_{j}^{+}(q(t, y)$ ), and $-\operatorname{Tr} L$ does not depend on $(t, y)$, cf. (7.15), (7.17).) Therefore, it remains to handle the interval $\Sigma_{j}$ around the $j^{\text {th }}$ soliton, cf. (7.43).

To this end we begin by exploiting the estimates (7.53), (7.55), (7.56), (7.51) and (7.52), as follows:

$$
\begin{aligned}
& \left|1 / \operatorname{ch} q_{j}-1 / \operatorname{ch} q_{j}^{\text {as }}\right|=\left|\operatorname{ch} q_{j}^{\text {as }}-\operatorname{ch} q_{j}\right| / \operatorname{ch} q_{j} \operatorname{ch} q_{j}^{\text {as }} \\
& \quad \leqslant 2 \exp \left(-\left|q_{j}\right|-\left|q_{j}^{\text {as }}\right|\right)\left(\left|\exp \left(q_{j}\right)-\exp \left(q_{j}^{\text {as }}\right)\right|+\left|\exp \left(-q_{j}\right)-\exp \left(-q_{j}^{\text {as }}\right)\right|\right) \\
& \quad<C \exp \left(-2 t \sigma_{j}|s|\right)\left(\exp \left[-t r_{j}^{+}(s)\right]+\exp \left[-t r_{j}^{-}(s)\right]\right) \\
& \quad<C \exp \left(-t R_{j}\right) .
\end{aligned}
$$

Hence, we need only show that $\left(\partial_{y} q_{j}+\sigma_{j}\right) / \operatorname{ch} q_{j}^{\text {as }}$ is $O\left(\exp \left(-t R_{j}\right)\right)$ on $\Sigma_{j}$ to complete the proof of (7.74). Since $q_{j}^{\text {as }}(t, y)$ has a zero on $\Sigma_{j}$, we need to majorize $\left|\partial_{y} q_{j}+\sigma_{j}\right|$.

It would be quite awkward to do so in the general setting of Th. 7.2, but here the particle Hamiltonians governing the space-time dependence have a simple relation to the Lax matrix, and with due labor this yields the desired estimate. First, since $H^{1}=\operatorname{Tr} L$ determines the $y$-dependence, we have (using also (7.9)) 


$$
\partial_{y} q_{j}+\sigma_{j}=-\exp \left(\theta_{j}\right) V_{j}^{+}(q)+\exp \left(\hat{\theta}_{j}\right)
$$

Now $\theta_{j}(t, y)$ is bounded on $\boldsymbol{R}^{2}$ (since the Hamiltonian $\Sigma \operatorname{ch} \theta_{j} V_{j}^{+}$is conserved), so we need only majorize $\left|V_{j}^{+}-1\right| / \operatorname{ch} q_{j}^{\text {as }}$ and $\left|\exp \left(\theta_{j}\right)-\exp \left(\hat{\theta}_{j}\right)\right| / \operatorname{ch} q_{j}^{\text {as }}$ on $\Sigma_{j}$.

To estimate $\left|V_{j}^{+}-1\right|$ we recall

$$
V_{j}^{+}(q)=\prod_{k \neq j} \operatorname{cth} \frac{1}{2}\left|q_{j}-q_{k}\right|
$$

cf. (1.3). Thus we have $0<V_{j}^{+}-1<V_{j}^{+2}-1$, and telescoping in the obvious way we deduce

$$
V_{j}^{+}(q)-1<C \max \left(\exp \left(q_{j}-q_{j-1}\right), \exp \left(q_{j+1}-q_{j}\right)\right) \quad\left(\text { on } \Sigma_{j}\right)
$$

Now from (7.53), (7.54) we have

$$
\max \left(\exp \left(q_{j}-q_{j-1}\right), \exp \left(q_{j+1}-q_{j}\right)\right)<C \exp \left[-t r_{j}(s)\right] \quad\left(\text { on } \Sigma_{j}\right)
$$

so that

$$
\begin{aligned}
\left|V_{j}^{+}(q)-1\right| / \operatorname{ch} q_{j}^{\text {as }} & <C \exp \left[-t r_{j}(s)-t \sigma_{j}|s|\right] \\
& <C \exp \left(-t R_{j}\right)
\end{aligned}
$$

where we used (7.51) for $s \in\left[0, s_{j}\right]$ and (7.52) for $s \in\left[-s_{j}, 0\right]$.

It remains to estimate $\left|\exp \left(\theta_{j}\right)-\exp \left(\hat{\theta}_{j}\right)\right|$ on $\Sigma_{j}$. To this end we first study $\theta_{j}\left(t, y_{j}(s, t)\right)$ as a function of $t$. From an inspection of $\tilde{A}\left(t, y_{j}(s, t)\right)$ (cf. (7.6), (7.9)) it follows that we may view the $t$-dependence as being generated by the Hamiltonian

$$
H_{j}(s) \equiv \frac{1}{3} \operatorname{Tr} L^{3}-\left(v_{j}+s\right) \operatorname{Tr} L
$$

cf. (6.5), (6.6), specialized to the case at hand. Now from the definition of $L$ (viz., (2.70) with $N_{+}=N, x^{+}, p^{+} \rightarrow q, 0, \beta=\mu=1, \tau=\pi / 2$ ) one readily sees that the following functions are bounded on $\boldsymbol{R}^{2}$, viewed as functions of $(t, y)$ : $\operatorname{ch} \frac{1}{2}\left(q_{k}-q_{l}\right) L_{k l}, \operatorname{ch} \frac{1}{2}\left(q_{j}-q_{k}\right) \partial_{q_{J}} L_{j k}$ and $\min \left(\exp \left(q_{j-1}-q_{j}\right), \exp \left(q_{j}-q_{j+1}\right)\right) \partial_{q_{J}} L_{m k}$ for $m=k$ and for $m, k \neq j$. Moreover, noting $L_{i j}=L_{j i}$, one obtains

$$
\dot{\theta}_{j}=\left\{\theta_{j}, H_{j}(s)\right\}=-\sum_{k, l, m} L_{k l} L_{l m} \partial_{q_{j}} L_{m k}+\left(v_{j}+s\right) \sum_{k} \partial_{q_{j}} L_{k k}
$$


A moment's thought then shows

$$
\left|\dot{\theta}_{j}\right|<C \max \left(\exp \left(q_{j}-q_{j-1}\right), \exp \left(q_{j+1}-q_{j}\right)\right) \quad\left(\text { on } \Sigma_{j}\right) .
$$

Next, we fix $s \in\left[-s_{j}, s_{j}\right]$ and combine (7.83) with (7.79) to infer

$$
\left|\dot{\theta}_{j}\left(u, y_{j}(s, u)\right)\right|<C \exp \left[-u r_{j}(s)\right], \quad u>\tilde{T}_{j}
$$

This estimate enables us to show that $\theta_{j}\left(T, y_{j}(s, T)\right)$ has a limit for $T \rightarrow \infty$. Indeed, since $r_{j}(s)>0$, the $r h s$ of the equality

$$
\theta_{j}\left(T, y_{j}(s, T)\right)-\theta_{j}\left(t, y_{j}(s, t)\right)=\int_{t}^{T} \dot{\theta}_{j}\left(u, y_{j}(s, u)\right) d u
$$

has a limit for $T \rightarrow \infty$, so that $\theta_{j}\left(T, y_{j}(s, T)\right)$ has a limit $\theta_{j}(\infty, s)$. Furthermore, (7.84) also entails

$$
\left|\theta_{j}(\infty, s)-\theta_{j}\left(t, y_{j}(s, t)\right)\right|<\frac{C}{r_{j}(s)} \exp \left[-t r_{j}(s)\right], \quad t>\tilde{T}_{j}
$$

so using (7.50) we may deduce

$$
\exp \left[\theta_{j}\left(t, y_{j}(s, t)\right)\right]-\exp \left[\theta_{j}(\infty, s)\right]=O\left(\exp \left[-\operatorname{tr}_{j}(s)\right]\right.
$$

Consequently, the desired estimate

$$
\left|\exp \left(\theta_{j}\right)-\exp \left(\hat{\theta}_{j}\right)\right| / \operatorname{ch} q_{j}^{\text {as }}=O\left(\exp \left(-t R_{j}\right)\right)
$$

follows just as (7.80) from (7.51) and (7.52), provided we can show $\theta_{j}(\infty, s)$ equals $\hat{\theta}_{j}$.

In order to prove this, we first show $\theta_{j}(\infty, s)$ does not depend on $s$. Indeed, we have

$$
\left|\partial_{y} \theta_{j}(t, y)\right|=\left|\sum_{k} \partial_{q_{j}} L_{k k}\right|<C \max \left(\exp \left(q_{j}-q_{j-1}\right), \exp \left(q_{j+1}-q_{j}\right)\right)
$$

which entails

$$
\left|\partial_{y} \theta_{j}\left(t, y_{j}(u, t)\right)\right|<C \exp \left(-t \tilde{r}_{j}\right), \quad u \in\left[-s_{j}, s_{j}\right]
$$


by virtue of (7.79) and (7.50). Integrating $\partial_{y} \theta_{j}$ we deduce

$$
\left|\theta_{j}\left(t, y_{j}(s, t)\right)-\theta_{j}\left(t, y_{j}^{0}\right)\right|<C|s| t \exp \left(-t \tilde{r}_{j}\right)
$$

and since the rhs goes to 0 for $t \rightarrow \infty, \theta_{j}(\infty, s)$ is equal to $\theta_{j}(\infty, 0)$.

It remains to prove $\theta_{j}(\infty, 0)=\hat{\theta}_{j}$. To this end we note (7.31) implies

$$
f(t) \equiv q_{j}\left(t, y_{j}^{0}\right)=O\left(\exp \left(-t r_{j}\right)\right)
$$

and study the rhs of

$$
\dot{f}(t)=\left\{q_{j}, H_{j}(0)\right\}=\sum_{k, l, m} L_{k l} L_{l m} \partial_{\theta_{j}} L_{m k}-v_{j} L_{j j}
$$

for $t \rightarrow \infty$. The matrix elements of $L\left(t, y_{j}^{0}\right)$ remain bounded and the function $\partial_{\theta_{j}} L_{m k}\left(t, y_{j}^{0}\right)$ converges to 0 unless $m=k=j$, in which case it has limit $\exp \left(\theta_{j}(\infty, 0)\right)$, cf. (2.70); moreover, the function $L_{j l}\left(t, y_{j}^{0}\right)$ has limit 0 unless $l=j$, in which case it has limit $\exp \left(\theta_{j}(\infty, 0)\right)$. The upshot is that we may conclude

$$
\lim _{t \rightarrow \infty} \dot{f}(t)=\exp \left(3 \theta_{j}(\infty, 0)\right)-\exp \left(2 \hat{\theta}_{j}+\theta_{j}(\infty, 0)\right)
$$

where we used (7.9). But in view of (7.92) $f(t)$ goes to 0 for $t \rightarrow \infty$, so that the rhs of (7.94) must vanish. From this we obtain $\hat{\theta}_{j}=\theta_{j}(\infty, 0)$, which completes the proof of the estimate $(1.14)_{+}$for the pure soliton solutions to the $\mathrm{mKdV}$ equation.

KdV. In this case we should prove

$$
\sup _{y \in \boldsymbol{R}} \mid \sum_{j=1}^{N}\left[-2 \partial_{y}^{2} \ln \left(1+\exp \left[q_{j}(t, y)\right]\right)+2 \partial_{y}^{2} \ln \left(1+\exp \left[q_{j}^{\text {as }}(t, y)\right]\right) \mid=O(\exp (-t r))\right.
$$

cf. (7.12), (7.14). Using (7.17) one readily verifies

$$
\partial_{y}^{2} q_{j}=2 \sum_{k \neq j} \exp \left(\theta_{k}+\theta_{j}\right) V_{k}^{+} \partial_{k} V_{j}^{+}
$$

Therefore, we should estimate the function 


$$
-4 \sum_{k \neq j} \frac{\exp \left(\theta_{k}+\theta_{j}\right) V_{k}^{+} \partial_{k} V_{j}^{+}}{1+\exp \left(-q_{j}\right)}-\frac{1}{2} \sum_{j}\left[\frac{\left(\partial_{y} q_{j}\right)^{2}}{\operatorname{ch}^{2} \frac{1}{2} q_{j}}-\frac{\sigma_{j}^{2}}{\operatorname{ch}^{2} \frac{1}{2} q_{j}^{\text {as }}}\right]
$$

Now the second sum can be handled in the same way as for $\mathrm{mKdV}$; one need only replace $(7.75)$ by

$$
\left|1 / \operatorname{ch}^{2} \frac{1}{2} q_{j}-1 / \operatorname{ch}^{2} \frac{1}{2} q_{j}^{\text {as }}\right| \leqslant \frac{1}{2}\left|\operatorname{ch}_{j}^{\text {as }}-\operatorname{ch}_{j}\right| / \operatorname{ch}^{2} \frac{1}{2} q_{j} \operatorname{ch}^{2} \frac{1}{2} q_{j}^{\text {as }}
$$

and proceed as before. Thus, it remains to estimate the first sum in (7.97). To this end we use (7.77) to rewrite the first sum as

$$
-4 \sum_{j>k} \exp \left(\theta_{k}+\theta_{j}\right) V_{k}^{+} V_{j}^{+} \frac{1}{\operatorname{sh}\left(q_{j}-q_{k}\right)}\left[\frac{1}{1+\exp \left(-q_{j}\right)}-\frac{1}{1+\exp \left(q_{k}\right)}\right]
$$

Now $\exp \left(\theta_{l}\right) V_{l}^{+}$is bounded on $\boldsymbol{R}^{2}$, so we need only estimate $\Sigma_{j>k} Q_{j k}$, where

$$
Q_{j k} \equiv 1 / 8 \operatorname{ch} \frac{1}{2}\left(q_{j}-q_{k}\right) \operatorname{ch} \frac{1}{2} q_{j} \operatorname{ch} \frac{1}{2} q_{k}<\exp \left[-\frac{1}{2}\left(q_{k}-q_{j}+\left|q_{j}\right|+\left|q_{k}\right|\right)\right]
$$

First, from (7.57) we deduce that $q_{j}$ is negative to the right of $\Sigma_{j}$ for $t>\hat{T}_{j} \geqslant \tilde{T}_{j}$, so that

$$
Q_{j k}<\exp \left(q_{j}\right)<C \exp \left(-t R_{j}\right), \quad s>s_{j}, \quad t>\hat{T}_{j}
$$

Second, we observe that the region to the left of $\Sigma_{j}$ is also to the left of $\Sigma_{k}$ for $t>T_{j k} \geqslant \tilde{T}_{k}$. Hence we may use (7.58) with $j \rightarrow k$ to conclude that $q_{k}$ is positive to the left of $\Sigma_{j}$ for $t>\hat{T}_{j k} \geqslant T_{j k}$, so that

$$
Q_{j k}<\exp \left[-q_{k}\left(t, y_{j}(s, t)\right)\right]<C \exp \left(-t R_{k}\right), \quad s<-s_{j}, \quad t>\hat{T}_{j k} .
$$

Finally, we estimate $Q_{j k}$ on $\Sigma_{j}$.

As $k \leqslant j-1$, one has

$$
q_{k} \geqslant q_{j-1}>-C+t d_{j, j-1}(s), \quad s \in\left[-s_{j}, s_{j}\right]
$$

in view of (7.54). Using $\left|q_{k}\right| \geqslant q_{k}$ and the bound (7.53), it readily follows from (7.100) that 


$$
Q_{j k}<C \exp \left[-t\left(d_{j, j-1}(s)+\frac{1}{2} \sigma_{j}[s+|s|]\right)\right], \quad s \in\left[-s_{j}, s_{j}\right]
$$

For non-positive $s$ this entails (recall (7.36))

$$
\begin{aligned}
Q_{j . k} & <C \exp \left[-t d_{j \cdot j-1}(s)\right] \\
& \leqslant C \exp \left[-t \sigma_{j-1}\left(v_{j-1}-v_{j}\right)\right]=O\left(\exp \left(-t r_{j}\right)\right), \quad s \in\left[-s_{j}, 0\right]
\end{aligned}
$$

whereas for non-negative $s$ we get

$$
Q_{j k}<C \exp \left[-t\left(\sigma_{j-1}\left(v_{j-1}-v_{j}\right)+s\left(\sigma_{j}-\sigma_{j-1}\right)\right)\right], \quad s \in\left[0, s_{j}\right]
$$

Recalling $\sigma_{j}=\exp \left(\hat{\theta}_{j}\right)<\exp \left(\hat{\theta}_{j-1}\right)=\sigma_{j-1}$ and $s_{j} \leqslant v_{j-1}-v_{j}$, (7.106) implies

$$
\begin{aligned}
Q_{j k} & <C \exp \left[-t\left(\sigma_{j-1}\left(v_{j-1}-v_{j}\right)+\left(v_{j-1}-v_{j}\right)\left(\sigma_{j}-\sigma_{j-1}\right)\right)\right] \\
& =C \exp \left[-t \sigma_{j}\left(v_{j-1}-v_{j}\right)\right]=O\left(\exp \left(-t R_{j}\right)\right), \quad s \in\left[0, s_{j}\right] .
\end{aligned}
$$

Combining the estimates (7.101), (7.102), (7.105) and (7.107) we may conclude

$$
Q_{j k}=O\left(\exp \left[-t \min \left(R_{j}, R_{k}\right)\right]\right)
$$

uniformly for $y \in \boldsymbol{R}$. As a result, the first sum in (7.97) has a modulus that is bounded above by $C(\exp (-t r))$, where $C$ depends only on the initial value $(\hat{q}, \hat{\theta})$. Thus, the $\mathrm{KdV} N$-soliton solutions are indeed approximated by a linear superposition of $N$ 1-soliton solutions according to the uniform exponential decay bound $(1.14)_{+}$.

Before turning to the general case we add three remarks. First, we would like to mention that the assumption (42) in our paper [2] entails the asymptotics (43) in [2] provided that the position $x$ in (43) (here denoted $y$ to avoid ambiguities) is assumed to be fixed. Indeed, this follows from Appendix C, and also from Th. A2 in I, since (42) implies $h_{0}^{\prime}\left(\hat{\theta}_{1}\right)<\cdots<h_{0}^{\prime}\left(\hat{\theta}_{N}\right)$. However, to obtain long-time asymptotics that is uniform in $y$, the key assumption is strict monotonicity of the velocity function $v(\theta) \equiv h_{0}^{\prime}(\theta) / h_{1}^{\prime}(\theta)$ (and not of $h_{0}^{\prime}(\theta)$ ), as will be clear from the above.

Second, we note that when one sets

$$
\sigma_{j}=-\operatorname{sh} \hat{\theta}_{j}, \quad v_{j}=\operatorname{cth} \hat{\theta}_{j}, \quad j=1, \cdots, N \quad \text { (sG; tachyons) }
$$


in (7.6), then

$$
\varphi \equiv 4 \operatorname{Tr} \operatorname{Arctg} \tilde{A}(t, y)-\pi N
$$

solves the sine-Gordon equation (7.1), provided $N$ is odd. (Indeed, (7.109) amounts to taking $t \leftrightarrow y$ in (7.6), as compared to (7.8).) Taking all $\hat{\theta}_{j}$ either positive or negative, the assumption (7.7) holds true up to overall signs. Thus, Th. 7.2 may be invoked to conclude that for odd $N$ such solutions are approximated by linear superpositions of $N$ 1-tachyon solutions according to the exponential decay bounds $(1.14)_{ \pm}, r$ being given by (7.71) with $\sigma_{i} \rightarrow\left|\sigma_{i}\right|$.

Third, combining (7.10)-(7.17) with (7.1)-(7.3) we can derive functional equations involving the potentials $V_{1}^{+}, \cdots, V_{N}^{+}$. Indeed, we can evaluate the partials in (7.1)-(7.3) by using Hamilton's equations. Doing so in the sine-Gordon case, we obtain the functional equation

$$
2 \sum_{j=1}^{N} \frac{\partial}{\partial q_{j}}\left[\frac{1}{\operatorname{ch} q_{j}} \prod_{k \neq j} \operatorname{cth}^{2} \frac{1}{2}\left(q_{j}-q_{k}\right)\right]=\sin \left(4 \sum_{j=1}^{N} \operatorname{Arctg}\left(\exp q_{j}\right)\right) .
$$

At first sight this identity looks utterly unlikely, and indeed a direct proof appears forbidding for $N>2$. It should be noted that our indirect proof hinges on the canonicity of the pure soliton map $\mathscr{E}$ established in I.

\section{B. Solitons, Antisolitons, and Breathers}

As announced, in this section we restrict attention to the sine-Gordon and modified $\mathrm{KdV}$ equations. Our starting point is the matrix $\tilde{A}(t, y)$ defined by (7.6), with the parameters $\sigma_{j}, v_{j}$ given by (7.8), (7.9), resp., and with $(\hat{q}, \hat{\theta}) \in \mathbb{R}^{N} \times G_{N}$. Since $\tilde{A}(t, y) \rightarrow 0$ for $y \rightarrow \infty$, we may write

$$
\operatorname{Tr} \operatorname{Arctg} \tilde{A}(t, y)=\frac{1}{2 i} \ln \left(\left|\mathbb{1}_{N}+i \tilde{A}(t, y)\right| /\left|\mathbb{1}_{N}-i \tilde{A}(t, y)\right|\right)
$$

provided that the logarithm is defined such that the rhs converges to 0 for $y \rightarrow \infty$. More explicitly, this can be rewritten

$$
\begin{aligned}
& \operatorname{Tr} \operatorname{Arctg} \tilde{A}(t, y)=-\frac{1}{2} i \ln \left(\left[1+\sum_{k=1}^{N} i^{k} S_{k}(\tilde{A}(t, y))\right] / \text { c.c. }\right) \\
& S_{k}=\sum_{|I|=k} \exp \left[\sum_{i \in I}\left(\hat{q}_{i}+\left[t \rho\left(\hat{\theta}_{i}\right)-y \sigma\left(\hat{\theta}_{i}\right)\right]\right)\right] \prod_{\substack{i \in I \\
j \notin I}} \operatorname{cth} \frac{1}{2}\left(\hat{\theta}_{\min (i, j)}-\hat{\theta}_{\max (i, j)}\right)
\end{aligned}
$$




$$
\rho(\theta) \equiv\left\{\begin{array}{ll}
\operatorname{sh} \theta \\
\exp 3 \theta
\end{array}, \quad \sigma(\theta) \equiv \begin{cases}\operatorname{ch} \theta & (\mathrm{sG}) \\
\exp \theta & (\mathrm{mKdV})\end{cases}\right.
$$

where (7.114) follows from (B4).

Consider now analytic continuation in $\hat{q}$ and $\hat{\theta}$. As long as we keep all $\hat{\theta}_{i}$ distinct and in the strip $\left|\operatorname{Im} \hat{\theta}_{i}\right|<\pi / 2$, we encounter no singularities in $S_{k}$ and retain exponential decay for $y \rightarrow \infty$. In particular, we may continue to the subset $\tilde{\hat{\Omega}}$ of $C^{2 N}$ defined by (4.20)-(4.24) and (6.132). (Recall $\tilde{\hat{\Omega}}$ depends on the choice of $N_{+}, N_{-}$; as before, this dependence will be suppressed.) Fixing $\tilde{\hat{P}} \in \tilde{\hat{\Omega}}$, we claim that the rhs of (7.113) defines a function $\psi(t, y)$ such that $4 \psi / \partial_{y} \psi$ is a real-valued real-analytic solution to the $s \mathrm{G} / \mathrm{mKdV}$ equation, resp.

To prove this claim, we first observe that $\tilde{A}(t, y)$ is diagonalizable and that we may write its spectrum as

$$
\sigma(\tilde{A}(t, y))=\left\{\exp \left[x_{1}^{+}(t, y)\right], \cdots,-\exp \left[x_{N_{-}}^{-}(t, y)\right]\right\}, \quad x_{1}^{\delta} \geqslant \cdots \geqslant x_{N_{\tilde{\delta}}}^{\delta}, \quad \delta=+,-
$$

(Recall (4.19), (2.56), (2.57) and the paragraph containing (2.84) to verify this.) Next, we infer from reality of $\sigma(\tilde{A})$ that the determinants $\left|\mathbf{1}_{N} \pm i \tilde{A}\right|$ are non-zero on $\boldsymbol{R}^{2}$. From the formulas (7.112)-(7.114) it is then plain that $\psi$ is real-valued and real-analytic on $\boldsymbol{R}^{2}$. Now the claim readily follows from the solution property for points in $\boldsymbol{R}^{N} \times G_{N}$ and real-analyticity of $\psi$. (Note that by picking $y$ large enough one can stay away from singularities while analytically continuing.)

Of course, one obtains the same solution for any two points in $\tilde{\hat{\Omega}}$ with the same image in $\hat{\Omega}$, cf. (4.19), (4.26). The solutions thus associated to points in $\hat{\Omega}$ are well known from the IST formalism. They all yield a vanishing reflection and a transmission coefficient with $N$ simple poles in the upper half plane. We are going to study a slightly larger class of solutions, considering successively the $\mathrm{sG}$ and $\mathrm{mKdV}$ cases. The details for the former case are presented in a format that enables us to dispose of the latter by simply listing some changes.

Firstly, we prove that for functions $\psi(t, y)$ associated to points in an open dense full measure subset $\hat{\Omega}_{l \neq}$ of $\hat{\Omega}_{l}$ one has 


$$
\begin{gathered}
\sup _{y \in \boldsymbol{R}}\left|\psi(t, y)-\sum_{i=1}^{k_{+}} \psi_{s_{i}}^{ \pm}(t, y)-\sum_{j=1}^{k-} \psi_{\bar{s}_{j}}^{ \pm}(t, y)-\sum_{n=1}^{l} \psi_{b_{n}}^{ \pm}(t, y)\right| \\
=O(\exp (-|t| r)), \quad t \rightarrow \pm \infty
\end{gathered}
$$

where $r>0$ will be specified below. In the $\mathrm{sG}$ case $4 \psi$ is a solution, and so are the soliton, antisoliton and breather functions $4 \psi_{s_{i}}^{ \pm}, 4 \psi_{\bar{s}_{j}}^{ \pm}$and $4 \psi_{b_{n}}^{ \pm}$; in the $\mathrm{mKdV}$ case the functions $\partial_{y} \psi, \partial_{y} \psi_{\ldots}^{ \pm}$are solutions. (Most likely, (7.117) holds true for the $y$-partials, too, but we have only proved this in the pure soliton case, cf. Section 7A.)

Secondly, we clarify the relation of the solutions corresponding to points in $\hat{\Omega}$ to the $\tau=\pi / 2 \tilde{\mathrm{I}}_{\mathrm{rel}}$ systems.

Thirdly, we use this relation to obtain and study a larger class of solutions associated to arbitrary points in the phase space $\Omega^{b}$.

Fourthly, we introduce soliton and antisoliton space-time trajectories and derive various properties therof.

Finally, in the sG case we add some comments, in particular on related literature.

Sine-Gordon. To take the first step in the program just delineated, we fix a point $\hat{P}_{\neq}$in the set

$$
\hat{\Omega}_{l \neq}=\left\{\hat{P} \in \hat{\Omega}_{l} \mid p_{1}^{s}, \cdots, p_{k_{+}}^{s}, p_{1}^{\bar{s}}, \cdots, p_{k_{-}}^{\bar{s}}, \frac{1}{2} p_{1}, \cdots, \frac{1}{2} p_{l} \text { distinct }\right\}
$$

(Note that $\hat{\Omega}_{0 \neq}=\hat{\Omega}_{0}$.) To determine the long-time asymptotics for the solution associated with $\hat{P}_{\neq}$, we follow a strategy that is an amalgam of the strategy in Section 7A, the asymptotic analysis in Chapter 6, and some new ingredients. We need some notation that will now be introduced and motivated.

First, note the bounds (7.50)-(7.52) in Th. 7.2 only involve properties of the functions (7.36)-(7.40). These functions depend solely on $s \in \boldsymbol{R}$ and on the positive numbers $\sigma_{1}, \cdots, \sigma_{N}$ and the distinct numbers $v_{N}<\cdots<v_{1}$. Here, we define numbers $\left(\sigma_{1}, v_{1}\right), \cdots,\left(\sigma_{k_{+}+l+k_{-}}, v_{k_{+}+l+k_{-}}\right)$with these properties by permuting the pairs $\left(\operatorname{ch} p_{1}^{s}, \operatorname{th} p_{1}^{s}\right), \cdots,\left(\operatorname{ch} \frac{1}{2} p_{1} \cos \frac{1}{2} \delta_{1}, \operatorname{th} \frac{1}{2} p_{1}\right), \cdots,\left(\operatorname{ch} p_{1}^{s}\right.$, th $\left.p_{1}^{s}\right), \cdots$ such that the velocities are in the desired order. Then the definitions (7.21), (7.36)-(7.40), (7.45), (7.46) make sense and the bounds (7.50)-(7.52) will entitle us to invoke Th. C1. 
Second, we denote by $\tau$ the permutation that reorders the actions $\left(a_{1}, \cdots, a_{N}\right)$ such that the associated velocities are decreasing from left to right, keeping the order of the two actions going with each breather. Thus we obtain a partition of the actions into subsets $J_{1}, \cdots, J_{k_{+}+l+k_{-}}$, such that $J_{\rho}$ contains the action(s) yielding velocity $v_{\rho}$. We define $J_{\rho}^{+}, J_{\rho}^{-}$via (C1), (C2), (C8) with $I \rightarrow J$.

Third, we define asymptotic single soliton/antisoliton/breather functions. To this end we fix $\lambda \in\left\{1, \cdots, k_{+}+l+k_{-}\right\}$and detail the three cases that may occur.

$$
\begin{aligned}
& \text { (s) } v_{\lambda}=\operatorname{th} p_{i}^{s} \\
& \psi_{s_{i}}^{ \pm} \equiv \operatorname{Arctg}\left(\exp \left[x_{i}^{s} \mp \frac{1}{2} \Delta_{i}^{s}+f_{i}(t, y)\right]\right) \\
& \Delta_{i}^{s} \equiv\left[\sum_{k \in J_{\lambda}^{+}}-\sum_{k \in J_{\bar{\lambda}}^{-}}\right] \operatorname{Re} \delta\left(p_{i}^{s}-a_{\tau^{-1}(k)}\right), \quad \delta(z) \equiv 2 \ln \left(\operatorname{cth} \frac{1}{2} z\right) \\
& f_{i} \equiv t \operatorname{sh} p_{i}^{s}-y \operatorname{ch} p_{i}^{s} \\
& (\bar{s}) \quad v_{\lambda}=\operatorname{th} p_{j}^{\bar{s}} \\
& \psi_{\bar{s}_{j}}^{ \pm} \equiv-\operatorname{Arctg}\left(\exp \left[x_{j}^{\bar{s}} \mp \frac{1}{2} \Delta_{j}^{\bar{s}}+\bar{f}_{j}(t, y)\right]\right) \\
& \Delta_{j}^{\tilde{s}} \equiv\left[\sum_{k \in J_{\lambda}^{+}}-\sum_{k \in J_{\lambda}^{-}}\right] \operatorname{Re} \delta\left(p_{j}^{\bar{s}}-a_{\tau}-1(k)\right) \\
& \bar{f}_{j} \equiv t \operatorname{sh} p_{j}^{\bar{s}}-y \operatorname{ch} p_{j}^{\bar{s}} \\
& \text { (b) } v_{\lambda}=\operatorname{th} \frac{1}{2} p_{n} .
\end{aligned}
$$

We first define real-valued functions $s_{n}^{ \pm}(t, y), \bar{s}_{n}^{ \pm}(t, y)$ by setting

$$
\begin{aligned}
& \frac{1}{2}\left[s_{n}^{ \pm}+\bar{s}_{n}^{ \pm}\right] \equiv x_{n} \mp \frac{1}{2} \Delta_{n}^{c}+f_{n}^{+}(t, y) \\
& \operatorname{sh} \frac{1}{2}\left[s_{n}^{ \pm}-\bar{s}_{n}^{ \pm}\right] \equiv\left|\cot \frac{1}{2} \delta_{n}\right| \sin \left(\gamma_{n} \mp \frac{1}{2} \Delta_{n}^{p}+f_{n}^{-}(t, y)\right)
\end{aligned}
$$


where $\Delta_{n}^{c}$ and $\Delta_{n}^{p}$ are real numbers such that

$$
\Delta_{n}^{c}+i \Delta_{n}^{p}=\left[\sum_{k \in J_{\lambda}^{+}}-\sum_{k \in J_{\lambda}^{-}}\right] \delta\left(\frac{1}{2}\left(p_{n}-i \delta_{n}\right)-a_{\tau}^{-1}(k)\right.
$$

and

$$
\begin{aligned}
& f_{n}^{+} \equiv\left(t \operatorname{sh} \frac{1}{2} p_{n}-y \operatorname{ch} \frac{1}{2} p_{n}\right) \cos \frac{1}{2} \delta_{n} \\
& f_{\mathrm{n}}^{-} \equiv\left(-t \operatorname{ch} \frac{1}{2} p_{n}+y \operatorname{sh} \frac{1}{2} p_{n}\right) \sin \frac{1}{2} \delta_{n}
\end{aligned}
$$

Then the asymptotic single breather functions read

$$
\psi_{b_{n}}^{ \pm} \equiv \operatorname{Arctg}\left(\exp \left[s_{n}^{ \pm}(t, y)\right]\right)-\operatorname{Arctg}\left(\exp \left[\bar{s}_{n}^{ \pm}(t, y)\right]\right)
$$

or, equivalently,

$$
\psi_{b_{n}}^{ \pm} \equiv \operatorname{Arctg}\left(\operatorname{sh} \frac{1}{2}\left[s_{n}^{ \pm}-\bar{s}_{n}^{ \pm}\right] / \operatorname{ch} \frac{1}{2}\left[s_{n}^{ \pm}+\bar{s}_{n}^{ \pm}\right]\right) .
$$

Note these functions correspond to points $\left(x_{n} \mp \frac{1}{2} \Delta_{n}^{c}, \gamma_{n} \mp \frac{1}{2} \Delta_{n}^{p}, p_{n}, \delta_{n}\right)$ in the $N_{+}=N_{-}=1$ manifold $\widetilde{B}_{1}$, cf. (4.10).

We are now in the position to state and prove the generalization of $(7.72)$.

Theorem 7.3. The functions $\psi_{s_{i}}^{\delta}, \psi_{\bar{s}_{j}}^{\delta}, \psi_{b_{n}}^{\delta}$ and

$$
\psi(t, y)=\sum_{i=1}^{N_{+}} \operatorname{Arctg}\left(\exp \left[x_{i}^{+}(t, y)\right]\right)-\sum_{j=1}^{N_{-}} \operatorname{Arctg}\left(\exp \left[x_{j}^{-}(t, y)\right]\right)
$$

associated to $\hat{P}_{\neq} \in \hat{\Omega}_{l \neq}$ via (7.120)-(7.122), (7.124)-(7.126), (7.128)-(7.133) and (7.112)-(7.116), resp., satisfy the decay estimates $(7.117)_{ \pm}$, the decay rate $r$ being given by

$$
r \equiv \min _{\rho . \lambda=1, \ldots, k_{+}+l+k_{-}} \sigma_{\rho}\left|v_{\rho}-v_{\lambda}\right|
$$


Proof. We only prove $(7.117)_{+}$, the proof of $(7.117)_{-}$being similar. In order to generalize the reasoning in Section $7 \mathrm{~A}$ we fix $\lambda \in\left\{1, \cdots, k_{+}+l+k_{-}\right\}$ and consider the eigenvalue(s) and asymptotic single particle function corresponding to the rays (7.35) with $j \rightarrow \lambda$. The real number $\zeta_{\lambda}$ now depends on whether one is dealing with a soliton, antisoliton or breather; specifically,

$$
\zeta_{\lambda} \equiv \frac{1}{\sigma_{\lambda}}\left\{\begin{array}{l}
\left(x_{i}^{s}-\frac{1}{2} \Delta_{i}^{s}\right) \\
\left(x_{j}^{\bar{s}}-\frac{1}{2} \Delta_{j}^{\bar{s}}\right) \\
\left(x_{n}-\frac{1}{2} \Delta_{n}^{c}\right) .
\end{array}\right.
$$

The definitions (7.21), (7.35)-(7.40), (7.42), (7.43), (7.45)-(7.49) will be in force from now on (taking $j \rightarrow \lambda$, of course), the numbers $C_{+, \lambda} / C_{-, \lambda}$ here denoting maximal/minimal eigenvalues of the positive matrix $M_{l}\left(0, \zeta_{\lambda}\right)$ defined via (6.14), (2.54).

We continue by specifying the generalization of (7.34). To this end we first set

$$
h(z) \equiv \operatorname{ch} z-\left(v_{\lambda}+s\right) \operatorname{sh} z
$$

Then we define a permutation $\sigma$ via the paragraph containing (6.22) (taking $\mu=1$ in (6.20)). Now we put

$$
E(t) \equiv \Pi_{\sigma} A_{s}\left(0, \zeta_{\lambda}\right) \operatorname{diag}\left(t h^{\prime}\left(a_{1}\right), \cdots, t h^{\prime}\left(a_{n}\right)\right) \Pi_{\sigma}^{-1} \sim \tilde{A}\left(t, y_{\lambda}(s, t)\right)
$$

and note that $E(t)$ is of the form (C4), with $M_{l}=M_{l}\left(0, \zeta_{\lambda}\right)$ in (6.24), $\mathscr{J}_{1}(t), \cdots, \mathscr{J}_{K}(t)$ of the form (6.25), and $d_{1}, \cdots, d_{K}$ defined through (6.20), (6.21).

Next, fixing $s \in\left[-s_{\lambda}, s_{\lambda}\right]$ and $t>\tilde{T}_{\lambda}$, the bounds (7.50)-(7.52) entail that Th. C1 applies, with $T_{\rho}, r_{\rho}, C_{\delta}$ and $d_{\rho}$ equal to $T_{\lambda}(s), r_{\lambda}(s), C_{\delta, \lambda}$ and $d_{\lambda \lambda}(s)=-\sigma_{\lambda} s$, resp. There are now three possibilities, namely the cases (7.119), (7.123) and (7.127), which correspond to (6.37), (6.40) and (6.43), resp.

In the first case (6.38) applies, yielding

$$
x_{i_{+}}^{+}\left(t, y_{\lambda}(s, t)\right)=-s t \operatorname{ch} p_{i}^{s}+O\left(\exp \left(-t r_{\lambda}(s)\right), \quad t \rightarrow \infty\right.
$$

(To check this, note that $\Delta_{i}^{s}$ defined by (6.39) and (6.119) coincides with $\Delta_{i}^{s}$ 
defined by (7.121), as anticipated by our notation. Indeed, one has

$$
I_{\rho}^{ \pm}=J_{\lambda}^{ \pm}, \quad \sigma^{-1}\left(I_{\rho}^{ \pm}\right)=\tau^{-1}\left(J_{\lambda}^{ \pm}\right)
$$

since $d_{\lambda i}(s) \lessgtr d_{\lambda \lambda}(s)$ for $i \lessgtr \lambda$.) Arguing now as in Section 7A we obtain

$$
\left|\operatorname{Arctg}\left(\exp \left[x_{i_{+}}^{+}\left(t, y_{\lambda}(s, t)\right)\right]\right)-\psi_{s_{i}}^{+}\left(t, y_{\lambda}(s, t)\right)\right|<C \exp \left(-t R_{\lambda}\right) .
$$

In the $(\bar{s})$ case $(6.141)$ can be used and we readily conclude

$$
\left|-\operatorname{Arctg}\left(\exp \left[x_{j_{+}}^{-}\left(t, y_{\lambda}(s, t)\right)\right]\right)-\psi_{\bar{s}_{j}}^{+}\left(t, y_{\lambda}(s, t)\right)\right|<C \exp \left(-t R_{\lambda}\right) .
$$

Finally, in the $(b)$ case we deduce by comparing (7.128) and (7.129) to (6.44) and (6.46) (with the substitutions (6.121) and $\gamma_{n} \rightarrow \gamma_{n}-\zeta_{\lambda} \operatorname{Im} h^{\prime}\left(\frac{1}{2}\left(p_{n}-i \delta_{n}\right)\right)$, of course)

$$
\begin{aligned}
& \mid \operatorname{Arctg}\left(\exp \left[x_{i_{+}^{+}}^{+}\left(t, y_{\lambda}(s, t)\right)\right]\right)-\operatorname{Arctg}\left(\exp \left[x_{j_{+}}^{-}\left(t, y_{\lambda}(s, t)\right)\right]\right) \\
& -\psi_{b_{n}}^{+}\left(t, y_{\lambda}(s, t)\right) \mid<C \exp \left(-t R_{\lambda}\right) .
\end{aligned}
$$

As before, the bound is uniform for $s \in\left[-s_{\lambda}, s_{\lambda}\right]$, since the constants $C_{\delta, \lambda}$ at the rhs of (C15) do not depend on $s$.

It remains to estimate the relevant differences for $y>y_{\lambda}^{+}$and $y<y_{\lambda}^{-}$. We claim that the functions $x_{k}^{\delta}(t, y), k=1, \cdots, N_{\delta}, \delta=+,-$, are all strictly decreasing in $y$. Accepting this claim for a while, it follows again that the differences satisfy uniform $O\left(\exp \left(-t R_{\lambda}\right)\right)$ tail bounds. For reasons of exposition we postpone the proof of the claim, cf. the paragraph containing (7.163).

We now come to the second step in the program sketched at the begining of this section. Fixing $\hat{P} \in \hat{\Omega}_{l}$, we define

$$
\mathscr{F}(t, y) \equiv \prod_{j<k}\left[\alpha_{j}(t, y)+\alpha_{k}(t, y)\right]^{2}
$$

where $\alpha_{1}, \cdots, \alpha_{N}$ are the eigenvalues of the matrix $\tilde{A}(t, y)$ associated with $\hat{P}$. We first assume $\hat{P}$ belongs to $\hat{\Omega}_{l}^{b}$. Then one has $\mathscr{F}(0,0) \neq 0$, so the zero locus of $\mathscr{F}$ is a real-analytic variety of dimension at most one. One can now repeat the reasoning leading up to $(6.103)$ to conclude 


$$
\tilde{A}(t, y) \sim A(t, y, P), \quad P \equiv \mathscr{E}_{l}(\hat{P}) \in \Omega_{l}^{b}
$$

where

$$
A(t, y, P) \equiv A(P) \exp \left[\frac{1}{2} t\left(L(P)-L(P)^{-1}\right)-\frac{1}{2} y\left(L(P)+L(P)^{-1}\right)\right] .
$$

(From here on it is convenient to make the $P$-dependence explicit.) Furthermore, we may and will view $\left(x^{+}(t, y), x^{-}(t, y)\right)$ as the position part of a piecewise orbit corresponding to the commuting Hamiltonians $H^{0}, H^{1}$. Denoting the zero locus of $\mathscr{F}$ by $C_{P}$ and the zero locus of

$$
\mathscr{D}(t, y) \equiv \prod_{j<k}\left[\alpha_{j}(t, y)-\alpha_{k}(t, y)\right]^{2}
$$

by $C_{P}^{m}$, it follows as in Section $6 \mathrm{C}$ that the positions are continuous on $\boldsymbol{R}^{2}$ and real-analytic off the multiple collision set $C_{P}^{m} \subset C_{P}$.

Consider next the non-generic case $\hat{P} \in \hat{\Omega}_{l} \backslash \hat{\Omega}_{l}^{p}$. Then $\mathscr{F}(0,0)=0$, so that $\mathscr{F}$ might vanish identically, a priori. We assert that this cannot happen. Taking this for granted, it follows that one may shift the space-time origin to some $\left(t_{0}, y_{0}\right)$ where $\mathscr{F}$ is non-zero; thus we may again view the sine-Gordon solution at hand as being associated with a point in the phase space $\Omega_{s}^{b}$. (Of course, the shift is not needed when one is willing to employ the extended phase space $\Omega_{s}^{\#}$, cf. the end of Section 6C.)

To prove the assertion, we choose $u \in R$ such that the $k_{+}+l+k_{-}$numbers $\operatorname{sh} p_{1}^{s}-u \operatorname{ch} p_{1}^{s}, \cdots,\left(\operatorname{sh} \frac{1}{2} p_{1}-u \operatorname{ch} \frac{1}{2} p_{1}\right) \cos \frac{1}{2} \delta_{1}, \cdots, \operatorname{sh} p_{1}^{\bar{s}}-u \operatorname{ch} p_{1}^{\bar{s}}, \cdots$ are distinct and such that the $l$ numbers $\operatorname{ch} \frac{1}{2} p_{1}-u \operatorname{sh} \frac{1}{2} p_{1}, \cdots$ are non-zero. Then we apply Th. C1 to (a similarity transform of) $\tilde{A}(t, u t)$ and deduce in a by now familiar way that the zero locus of $\mathscr{F}$ meets the ray $y=u t$ in a discrete set.

We proceed with the third step. To this end we observe that the definition (7.147) of $A(t, y, P)$ makes sense for any $P \in \Omega^{b}$; denoting the roots of $\left|A(t, y, P)-\alpha \mathbf{1}_{N}\right|$ by $\alpha_{1}, \cdots, \alpha_{N}$ we define functions $\mathscr{F}$ and $\mathscr{D}$ by (7.145) and (7.148), and we denote their zero loci by $C_{P}$ and $C_{P}^{m}$, resp. The arguments yielding Th. 6.7 can now be adapted to the 2-parameter flows at issue. Thus, through any $P \in \Omega^{b}$ there passes a piecewise orbit that is the limit of the global orbit $\boldsymbol{R}^{2} \rightarrow \Omega,(t, y) \mapsto \exp \left(t H^{0}(g)-y H^{1}(g)\right)(P)$ as $g \uparrow \pi$. (The Hamiltonians $H^{\mu}(g)$ are given by (7.16) with $L=L(g)$, of course.) Moreover, $A(t, y, P)$ has real spectrum 
on $\boldsymbol{R}^{2}$ and simple spectrum off $C_{P}^{m}$; for $(t, y) \in \boldsymbol{R}^{2} \backslash C_{P}$ its eigenvalues are related to the configuration part of the piecewise orbit via (7.116), and the position $x_{k}^{\delta}(t, y)$ has a continuous extension to $\boldsymbol{R}^{2}$ that is real-analytic wherever the corresponding eigenvalue is simple (in particular, off $C_{P}^{m}$ ). We are now in the position to state and prove our next theorem.

Theorem 7.4. The function $\varphi(t, y) \equiv 4 \psi(t, y)$ associated to $P \in \Omega^{b}$ via (7.135) is a real-valued and real-analytic solution to the sine-Gordon equation (7.1). It satisfies

$$
\lim _{y \rightarrow \infty} \varphi(t, y)=0, \quad \lim _{y \rightarrow-\infty} \varphi(t, y)=2 \pi\left(N_{+}-N_{-}\right)
$$

and

$$
\left(\partial_{y} \varphi\right)(t, \cdot) \in S(\boldsymbol{R})
$$

where $S(\boldsymbol{R})$ denotes Schwartz space. Moreover, the solution yields a reflectionless potential in the sense of the IST.

Proof. For $P \in \Omega_{s}^{b}$ all of these properties are well known, cf. the beginning of this section. Now let $P \in \Omega_{e}^{b}$. Since $\Omega_{s}^{b}$ is dense in $\Omega^{b}$, we can find a sequence $P_{n} \in \Omega_{s}^{b}$ converging to $P$. The corresponding solutions may be written

$$
\varphi_{n}(t, y)=-2 i \ln \left(\left|\mathbf{1}_{N}+i A\left(t, y, P_{n}\right)\right| / \text { c.c. }\right)
$$

or, equivalently,

$$
\varphi_{n}(t, y)=-2 i \ln \left(\left[1+\sum_{k=1}^{N} i^{k} S_{k}\left(A\left(t, y, P_{n}\right)\right)\right] / \text { c.c. }\right)
$$

cf. (7.112), (7.113). Since $A(\cdot, \cdot, \cdot)$ is real-analytic and has real spectrum on $\boldsymbol{R}^{2} \times \Omega^{b}$, these representations entail that $\varphi_{n}(t, y)$ converges (uniformly on compacts) to a real-valued real-analytic sG solution. This solution equals $\varphi(t, y)$ as defined by (7.135), since (7.135) entails that $\varphi$ may also be written

$$
\varphi(t, y)=4 \operatorname{Tr} \operatorname{Arctg} A(t, y, P)=-2 i \ln \left(\left|\mathbb{1}_{N}+i A(t, y, P)\right| / \text { c.c. }\right) .
$$

Next, we choose a closed contour $\Gamma$ in the open right half plane that encircles the spectrum of $L(P)$ counterclockwise. Then the functional calculus yields 


$$
M(y, P) \equiv \exp \left[-\frac{1}{2} y\left(L(P)+L(P)^{-1}\right)\right]=\frac{1}{2 \pi i} \int_{\Gamma} \exp \left[-\frac{1}{2} y\left(z+z^{-1}\right)\right] R(z, L(P)) d z
$$

where $R$ denotes the resolvent. Since $R$ is continuous on $\Gamma$, its norm is bounded on $\Gamma$. But now it readily follows that all $y$-derivatives of any matrix element $M(y, P)_{j k}$ have decay $\exp (-d y)$ for any $d$ smaller than

$$
\sigma_{-} \equiv \min _{\lambda \in \sigma(L(P))} \operatorname{Re}\left(\lambda+\lambda^{-1}\right) / 2
$$

In view of (7.147) the same holds true for $A(t, y, P)$. From (7.153) it is then clear that $\left(\partial_{y}^{k} \varphi\right)(t, y), k \geqslant 0$, has decay $\exp (-d y)$ for $y \rightarrow \infty$. Rewriting $\varphi$ as

$$
\begin{aligned}
\varphi(t, y) & =2 \pi\left(N_{+}-N_{-}\right)-4 \sum_{\delta=+,-} \delta \sum_{k=1}^{N_{\delta}} \operatorname{Arctg}\left(\exp \left[-x_{k}^{\delta}(t, y)\right]\right) \\
& =2 \pi\left(N_{+}-N_{-}\right)-4 \operatorname{Arctg}\left(A(t, y, P)^{-1}\right)
\end{aligned}
$$

and repeating the reasoning for $A(t, y, P)^{-1}$, we may now deduce (7.149) and (7.150).

Finally, we claim

$$
\lim _{n \rightarrow \infty} \int_{\boldsymbol{R}}\left|\varphi_{n}(t, y)-\varphi(t, y)\right| d y=0
$$

Taking this for granted, it is not hard to prove the last assertion. Indeed, the IST $S$-matrix can be defined via a Dyson series/Volterra expansion/variation of constants formula/product integral/time-ordered exponential (choose your favorite), $y$ playing the role of time. (Cf. in this connection [12], pp. 394-399, especially Eqs. (4.1)-(4.4), (4.49), (4.52).) The crux is now that (7.157) ensures $L^{1}(\boldsymbol{R})$-convergence of the associated potential difference $V_{n}(t, \cdot)-V(t, \cdot)$; in turn, this entails convergence of the $S$-matrix, as is readily verified. Since $V_{n}$ is reflectionless, so is $V$.

It remains to prove (7.157). Since the integrand converges to 0 pointwise for $n \rightarrow \infty$, we need only dominate $\left|\varphi_{n}-\varphi\right|$ by an $L^{1}$-function. To this end, choose $n_{0}$ large enough so that $\sigma\left(L\left(P_{n}\right)\right)$ is enclosed by $\Gamma$ for $n>n_{0}$. Then 
(7.154) holds true when $P$ is replaced by $P_{n}, n>n_{0}$. Fixing $d \in\left(0, \sigma_{-}\right)$, it now easily follows one can find $C_{+}>0$ such that $\left|\varphi_{n}(t, y)\right|<C_{+} \exp (-d y)$ for any $n>n_{0}$ and $y \geqslant 0$, say. Taking $y \rightarrow-y$ in (7.154) and using the representation (7.156), one infers in the same way $\left|\left(\varphi_{n}-\varphi\right)(t, y)\right|<C_{-} \exp (d y)$ for any $n>n_{0}$ and $y \leqslant 0$.

We have little doubt that the class of sine-Gordon solutions obtained via the $\tau=\pi / 2 \tilde{\mathrm{I}}_{\mathrm{rel}}$ systems coincides with the class of all real-valued reflectionless solutions satisfying (7.149) $(\bmod 2 \pi$, of course) and (7.150). More precisely, we believe that any solution from the latter class whose transmission coefficient has $N$ poles (counting multiplicities) in the open upper half plane can be obtained from a point in $\Omega^{b}$, possibly after a translation of the space-time origin. In particular, the multipole solutions [13] correspond to points in the exceptional set $\Omega_{e}^{b}$ in this scenario (which is easily verified for $N=2$ ).

However, having a proof is far better than having no doubt. We expect that a proof would reveal features of the exceptional set and of the multipole solutions that are hard to arrive at when one stays within one of the two contexts that are involved.

We now come to the fourth item announced above, viz., soliton and antisoliton space-time trajectories. To define these, we introduce the trajectory function

$$
\begin{aligned}
\mathscr{T}(t, y, P) & \equiv\left|\mathbb{1}_{N}-A(t, y, P)^{2}\right| \\
& =\prod_{i=1}^{N_{+}}\left(1-\exp \left[2 x_{i}^{+}(t, y)\right]\right) \prod_{j=1}^{N_{-}}\left(1-\exp \left[2 x_{j}^{-}(t, y)\right]\right) .
\end{aligned}
$$

Obviously, $\mathscr{T}$ is real-analytic on $\mathbb{R}^{2} \times \Omega^{b}$. Since $A(t, y, P) \rightarrow 0$ for $y \rightarrow \infty$, one has $\mathscr{T}(\cdot, \cdot, P) \neq 0$. Thus, the zero locus $T_{P}$ of $\mathscr{T}(\cdot, \cdot, P)$ is a real-analytic variety of dimension at most one, just as the zero loci $C_{P}$ and $C_{P}^{m}$ of the functions $\mathscr{F}$ and $\mathscr{D}$ defined by (7.145) and (7.148), resp.

We shall now study the trajectory set $T_{P}$ in more detail, in particular as concerns its relation to the collision sets $C_{P}$ and $C_{P}^{m}$. Before embarking on this, let us specify the local structure any of these varieties $V_{P}$ can have, a priori: A point $\left(t_{0}, y_{0}\right) \in V_{P}$ can be either isolated or belong to finitely many real-analytic curves, some of which may end at $\left(t_{0}, y_{0}\right)$. (This readily follows from the Weierstrass preparation theorem.)

We begin by noting that the trajectory sets are Lorentz equivariant. That is, we have actions of the Lorentz group $\boldsymbol{R}$ both on space-time $\boldsymbol{R}^{2}$ (sending $(t, y)$ to 
$\left.(t, y)_{\theta} \equiv(t \operatorname{ch} \theta-y \operatorname{sh} \theta, y \operatorname{ch} \theta-t \operatorname{sh} \theta)\right)$ and on $\Omega^{b}$ (sending $P=(x, p)$ to $P(\theta)$ $\left.\equiv\left(x, p_{1}^{+}+\theta, \cdots, p_{N_{-}}^{-}+\theta\right)\right)$, such that the image of $T_{P}$ under the former action equals $T_{P(-\theta)}$. (Indeed, this is clear from $\mathscr{T}\left((t, y)_{\theta}, P\right)=\mathscr{T}(t, y, P(\theta))$, cf. (7.158), (7.147), (2.70).) For space-time translations the situation is different, since $H^{0}$ and $H^{1}$ give rise to collisions. However, when we employ the extended phase space $\Omega^{\sharp}$, we do obtain an $\boldsymbol{R}^{2}$-action $Q \mapsto Q(a) \equiv \exp \left(a^{0} H^{0 \#}-a^{1} H^{1 \#}\right)(Q)$. For points in $\Omega_{s}^{\#} \simeq \hat{\Omega}$ it is then not hard to establish Poincaré equivariance, but for $Q \notin \Omega_{s}^{\#}$ we get trouble with Lorentz boosts. Indeed, it is clear how the latter should be extended from $\hat{\Omega}^{b}$ to $\hat{\Omega}$, and they also have an obvious (and compatible) definition on the pair set $\mathscr{P}$, viz., $(A, L) \mapsto\left(A, e^{\theta} L\right)$. But when $\sigma(L)$ is not simple, we cannot be sure that the latter definition gives rise to an action on $\Omega^{\sharp}$, cf. the open questions discussed at the end of Section $6 \mathrm{C}$. This is immaterial for what follows, however.

Next, we define

$$
v_{ \pm} \equiv \max _{\lambda \in \sigma(L(P))} \frac{\operatorname{Re} \lambda-\operatorname{Re} \lambda^{-1}}{\operatorname{Re} \lambda+\operatorname{Re} \lambda^{-1}} \in(-1,1)
$$

and consider rays $\left(t_{0}, y_{0}\right)+r(\cos \xi, \sin \xi), r \in[0, \infty)$, in the four regions complementing the two lines $\left(t_{0}, y_{0}\right)+\mu\left(1, v_{ \pm}\right), \mu \in \boldsymbol{R}$. For rays in the region containing the point $\left(t_{0}, y_{0}+1\right)$ we may use the functional calculus as in the proof of Th. 7.4 to conclude $A\left(t_{0}+r \cos \xi, y_{0}+r \sin \xi, P\right) \rightarrow 0$ for $r \rightarrow \infty$. Similarly, along rays in the region containing $\left(t_{0}, y_{0}-1\right)$, the matrix $A^{-1}$ vanishes. Therefore, as $t \rightarrow \pm \infty$, the set $T_{P} \cap\{(t, y) \mid y \in \boldsymbol{R}\}$ moves into any open timelike cone that contains the closed timelike cone spanned by the above two lines, and, a fortiori, into the forward and backward light cones as $t \rightarrow \infty$ and $t \rightarrow-\infty$, resp. Moreover, recalling (7.116), we deduce

$$
\lim _{y \rightarrow \pm \infty} x_{k}^{\delta}\left(t_{0}+u y, y\right)=\mp \infty, \quad u \in[-1,1]
$$

Fixing $\left(t_{0}, u\right) \in \boldsymbol{R} \times[-1,1]$, we now claim that the functions $y \mapsto x_{k}^{\delta}\left(t_{0}+u y, y\right)$ are strictly decreasing. We have already made this claim in a special case, cf. the end of the proof of Th. 7.3. Accepting it a little longer, we may invoke (7.160) to infer that the equations

$$
x_{i}^{+}(t, y)=0, \quad i=1, \cdots, N_{+}, \quad x_{j}^{-}(t, y)=0, \quad j=1, \cdots, N_{-}, \quad t \text { fixed }
$$

admit unique solutions $y_{i}^{+}(t)$ and $y_{j}^{-}(t)$, which we shall refer to as soliton and 
antisoliton space-time trajectories, resp. In addition, it now follows that we may write

$$
T_{P}=\bigcup_{\delta=+,-} \bigcup_{k=1, \cdots, N_{\delta}}\left\{\left(t, y_{k}^{\delta}(t)\right) \mid t \in \boldsymbol{R}\right\}
$$

and that trajectory tangents cannot be spacelike.

At last, we prove our claim. First, we note it suffices to show $x_{k}^{\delta}\left(t_{0}+u y, y\right)$ is decreasing in $y$. (Indeed, the assumption $x_{k}^{\delta}\left(t_{0}+u y, y\right)=c$ on a $y$-interval entails that the line $\left\{\left(\delta e^{c}, y\right) \mid y \in \boldsymbol{R}\right\}$ belongs to the zero locus of the function $(\alpha, y) \mapsto\left|\alpha \mathbb{1}_{N}-A\left(t_{0}+u y, y, P\right)\right|$, contradicting $A \rightarrow 0$ for $y \rightarrow \infty$.) Suppose $x_{k}^{\delta}\left(t_{0}\right.$ $\left.+u y_{1}, y_{1}\right)>x_{k}^{\delta}\left(t_{0}+u y_{2}, y_{2}\right)$ for $y_{1}>y_{2}$. Choose a sequence $t_{n} \rightarrow t_{0}$ such that each of the lines $\left\{\left(t_{n}+u y, y\right) \mid y \in \mathbb{R}\right\}$ meets $C_{P}$ in a discrete set. From Hamilton's equations one has

$$
\partial_{y}\left(x_{k}^{\delta}(t+u y, y)\right)=\left[\left(u \operatorname{sh} p_{k}^{\delta}-\operatorname{ch} p_{k}^{\delta}\right) V_{k}^{\delta}\right](t+u y, y)<0, \quad(t+u y, y) \in \mathbb{R}^{2} \backslash C_{P}
$$

so that $x_{k}^{\delta}\left(t_{n}+u y_{1}, y_{1}\right)<x_{k}^{\delta}\left(t_{n}+u y_{2}, y_{2}\right)$. But then it follows by continuity that $x_{k}^{\delta}\left(t_{0}+u y_{1}, y_{1}\right) \leqslant x_{k}^{\delta}\left(t_{0}+u y_{2}, y_{2}\right)$, a contradiction. Hence, the claim is finally proved.

Returning now to our study of the trajectories, consider the zero locus of the real-analytic example function

$$
E(t, y) \equiv y\left(\operatorname{sh}^{2} y+(t+T)^{3}\right)\left(\operatorname{sh}^{2} y-(t-T)^{3}\right), \quad T>0 .
$$

It has all of the properties of $T_{P}$ established thus far, so we need additional arguments to exclude the contingency that there are fewer than $N$ trajectories for a non-zero time interval (a phenomenon that would correspond to resonances, physically speaking).

We conjecture this behavior never occurs, but have not found a complete proof. As will be clear from what follows, it would suffice to show that the multiple collision set $C_{P}^{m}$ is discrete for any $P \in \Omega^{b}$. (We believe this is true.) Before studying the general case in some detail, let us mention that the special point $P$ given by (6.116) yields $C_{P}^{m}=\{(T, 0)\}$ for $P_{0}(x, p) \geqslant 3$ and a discrete $C_{P}^{m}$ on the $t$-axis for $P_{0}<3$. It is obvious that the antisoliton trajectory is given by the $t$-axis. Moreover, the three trajectories meet only in $C_{P}^{m}$ and the soliton trajectory tangents are lightlike in the triple collision point(s). (The verification 
of these assertions is straightforward.)

We shall now elucidate the local structure of the 'regular collision' set

$$
R_{P} \equiv C_{P} \backslash C_{P}^{m}, \quad P \in \Omega^{b}
$$

To this end, let $b=\left(t_{0}, y_{0}\right) \in R_{P}$. We assert that the intersection of $R_{P}$ with a sufficiently small disc around $b$ consists of $l$ spacelike curves meeting at $b$, where $l \in\{1, \cdots, m\}$. Taking this assertion for granted, it follows that $T_{P}$ can meet $R_{P}$ only in a discrete set. (Indeed, as shown above, trajectory tangents can only be timelike or lightlike.) In particular, it follows that whenever $C_{P}^{m}$ is discrete, there are $N$ distinct trajectories for any bounded time interval, except possibly for a finite set of times.

We now prove the assertion about $b \in R_{P}$. We begin by noting there are at least one pair and at most $m$ pairs of soliton and antisoliton positions coinciding at $b$. Let $x_{i}^{+}(t, y), x_{j}^{-}(t, y)$ be one of these pairs. Since $\sigma(A(b))$ is simple, $x_{i}^{+}-x_{j}^{-}$is real-analytic at $b$. Now choose a line segment

$$
S \equiv\left\{b+s(\cos \xi, \sin \xi) \equiv b_{s} \mid s \in(0, \delta)\right\}
$$

such that $S$ does not meet $C_{P}$. (Using real-analyticity of $\mathscr{F}(t, y)$ and $C_{P} \neq \boldsymbol{R}^{2}$ one sees that such an $S$ exists.) Then we have from Hamilton's equations

$$
\partial_{t}\left[x_{i}^{+}(t, y)-x_{j}^{-}(t, y)\right]=\left(\operatorname{sh} p_{i}^{+} V_{i}^{+}-\operatorname{sh} p_{j}^{-} V_{j}^{-}\right)\left(b_{s}\right), \quad(t, y)=b_{s} \in S .
$$

To get information on the limit of the $r h s$ as $s \rightarrow 0$ (which exists, since the lhs is smooth at $b$ ), we exploit Th. 6.7 with $h(z) \equiv \cos \xi \operatorname{ch} z-\sin \xi \operatorname{sh} z$. First, we use (6.112) to infer that the limit of the $r h s$ is finite. Thus the gradient of $x_{i}^{+}-x_{j}^{-}$does not vanish at $b$, entailing that the zero locus of $x_{i}^{+}-x_{j}^{-}$is a smooth curve through $b$ (sufficiently near $b$, of course).

It remains to prove that the tangent at $b$ to this curve is spacelike. To this end we rewrite (7.167) as

$$
\begin{aligned}
\partial_{t}\left(x_{i}^{+}-x_{j}^{-}\right)= & {\left[2 \operatorname{sh} \frac{1}{2}\left(p_{i}^{+}-p_{j}^{-}\right) \operatorname{ch} \frac{1}{2}\left(p_{i}^{+}+p_{j}^{-}\right) V_{i}^{+}\right.} \\
& \left.-\operatorname{sh} p_{j}^{-} V_{j}^{-}\left(1-V_{i}^{+} / V_{j}^{-}\right)\right]\left(b_{s}\right) .
\end{aligned}
$$

Now from (1.3) and (1.4) one reads off $V_{i}^{+} / V_{j}^{-} \rightarrow 1$ as $s \rightarrow 0$. Combining this with (6.112), it follows that the second term at the rhs goes to 0 as $s \rightarrow 0$. Telescoping $\partial_{y}\left(x_{i}^{+}-x_{j}^{-}\right)$in the same fashion, we readily deduce 


$$
\lim _{s \rightarrow 0} \partial_{y}\left(x_{i}^{+}-x_{j}^{-}\right) / \partial_{t}\left(x_{i}^{+}-x_{j}^{-}\right)=\lim _{s \rightarrow 0} \operatorname{th} \frac{1}{2}\left(p_{i}^{+}+p_{j}^{-}\right)\left(b_{s}\right) \equiv \operatorname{th} p_{0} \in(-1,1) .
$$

From this it is plain that the tangent at $b$ is spacelike, so our assertion is proved.

We are now prepared to study the local structure of $T_{p}$. Fixing $b \equiv\left(t_{0}, y_{k}^{\delta}\left(t_{0}\right)\right)$, there are three possibilities. First, no $x_{k^{\prime}}^{\delta^{\prime}}(t, y)$ other than $x_{k}^{\delta}$ vanishes at $b$. Then one can find $\epsilon>0$ such that no position but $x_{k}^{\delta}$ vanishes on the disc with radius $\epsilon$ around $b$, and such that $x_{k}^{\delta}$ stays away from the other positions on this disc. Since $x_{k}^{\delta}$ is strictly decreasing in $y$ and real-analytic on the disc, its zero locus $\left(t, y_{k}^{\delta}(t)\right)$ in the disc is a real-analytic curve. We claim that this curve is timelike.

To prove this, we assume first $b \notin C_{P}$. Then we may use Hamilton's equations

$$
\left(\partial_{t} x_{k}^{\delta}\right)(b)=\left(\operatorname{sh} p_{k}^{\delta} V_{k}^{\delta}\right)(b), \quad\left(\partial_{y} x_{k}^{\delta}\right)(b)=\left(-\operatorname{ch} p_{k}^{\delta} V_{k}^{\delta}\right)(b)
$$

to infer that $\left(d y_{k}^{\delta} / d t\right)\left(t_{0}\right)=$ th $p_{k}^{\delta}(b)$, so the curve is timelike at $b$. Next, assuming $b \in C_{P}$, let $S$ be a line segment of the form (7.166) which is not in $C_{P}$. On $S$ one has (7.170) with $b \rightarrow b_{s}$; since $x_{k}^{\delta}$ is real-analytic at $b$ and $V_{k}^{\delta}\left(x\left(b_{s}\right)\right)$ has the finite limit $V_{k}^{\delta}(x(b))$ as $s \rightarrow 0$, it follows that $p_{k}^{\delta}\left(b_{s}\right)$ has a limit $p_{k}^{\delta}(b) \in \boldsymbol{R}$; hence, we arrive at the same conclusion as for $b \notin C_{P}$.

The second possibility is that both $x_{k}^{\delta}$ and some $x_{k^{\prime}}^{-\delta}$ vanish at $b$, but no other position does. Then the corresponding eigenvalues are simple at $b$ and hence remain simple on a disc around $b$ with sufficiently small radius $\epsilon$. Eventually shrinking $\epsilon$, we may assume no other position vanishes on this disc. Thus we obtain two real-analytic curves $\left(t, y_{k}^{\delta}(t)\right),\left(t, y_{k^{\prime}}^{-\delta}(t)\right)$ in the disc that have at least one point in common, namely $b \in C_{P}$. Choosing a segment $S$ of the form (7.166) that is not in $C_{P}$, we have (7.170) with $b \rightarrow b_{s}$. Exploiting now Th. 6.7 in the same way as before, it follows that the curves have lightlike tangents at $b$ with opposite slopes. In particular, the curves separate as $t$ moves away from $t_{0}$.

The third case is that more than two trajectories meet at $b$, implying $b \in C_{P}^{m}$. Whenever $b$ is an isolated point of $C_{P}^{m}$, it follows from the above that all of these trajectories separate as $t$ moves away from $t_{0}$. (But we have no general information on slopes. Are there always lightlike tangents at $b$, just as for the point (6.116)?) However, since we have not succeeded in proving non-existence of non-spacelike curves in $C_{P}^{m}$, some trajectories might coincide as $t$ moves away from $t_{0}$.

We conclude our study of the trajectories by adding some observations 
that have a bearing on this 'multiple trajectory' phenomenon. Firstly, if it exists at all, it is non-generic. For instance, one can consider along with $P$ the points $P(d)$ obtained from $P$ by translating all positions over the distance $d$. Since the union of the sets $T_{P(d)}$ as $d$ varies over $\boldsymbol{R}$ is equal to $\boldsymbol{R}^{2}$ (recall (7.158) and (7.147) to see this), and since one clearly has $C_{P(d)}^{m}=C_{P}^{m}$, it follows that multiple trajectories can only occur for a discrete set of $d$.

Secondly, for $P \in \Omega_{s}^{b}$ with image $\hat{P} \in \hat{\Omega}_{l \neq}$ it is readily seen from the proof of Th. 7.3 that for $t \rightarrow \pm \infty$ one gets $N$ distinct trajectories, save for the times at which the soliton and antisoliton in any of the $l$ breathers collide. More specifically, one infers (cf. e.g. (7.140)) that for $t \rightarrow \delta \infty$ one gets $k_{+}+k_{-}$trajectories at the extremum of the functions $y \mapsto \partial_{y} \psi_{\ldots}^{\delta}(t, y)$ given by (7.120) and (7.124), and two trajectories associated with each $\psi_{b_{n}}^{\delta}(t, y)$, whose location follows from (7.128)-(7.133) (up to an exponentially small error).

An interesting (and puzzling) feature of the two breather trajectories is that their maximal distance has a finite limit as $\delta$ goes to its minimum $-\pi$. To be specific, for a breather with $p=0$ (corresponding to its rest frame) one easily checks that the maximal distance between the trajectories reads

$$
d_{\max }=\frac{2}{\cos (\delta / 2)} \operatorname{Arth}(\cos (\delta / 2)), \quad \delta \in(-\pi, 0), \quad p=0
$$

Thus, $d_{\max }$ decreases monotonically from $\infty$ to 2 as $\delta$ goes from 0 to $-\pi$. It is to be noted that $d_{\max } \cos \delta / 2$ equals the distance between the two underlying point particles at their turning point and that the oscillation periods of the breather solution and of its associated trajectories and particle motion are equal (viz., $2 \pi /|\sin \delta / 2|$ ).

We conclude our account of the sine-Gordon case with several remarks.

(i) The asymptotic behavior detailed in Th. 7.3 is in accordance with pp. 429-431 in [12]. More precisely, our decay bounds do not contradict 1.c. Eq. (5.151), the qualification being necessitated by the fact that the analytical meaning of Eq. (5.151) is left unspecified. (From the arguments presented on pp. 133-134 of [12] it does not even follow that the lhs of (1.14) goes to 0 for $t \rightarrow \pm \infty$.)

(ii) The above trajectories can also be viewed as trajectories associated to singular solutions of the sinh-Gordon equation, cf. the review [17] and references therein. (This was pointed out to us by Pogrebkov [20].) Indeed, taking $\hat{q}_{j} \rightarrow \hat{q}_{j}+i \pi / 2$ in $(7.4)-(7.6)$, one obtains $\tilde{A}(t, y) \rightarrow i \widetilde{A}(t, y)$. Combining this with 
(7.146) and (7.153), one infers that the function $2 \ln \left(\left|\mathbb{1}_{N}+A(t, y, P)\right| /\left|\mathbb{1}_{N}-A(t, y, P)\right|\right)$ solves the sinh-Gordon equation. Clearly, this solution is singular along isolated trajectories, cf. (7.158). (But singularities might cancel when multiple trajectories do occur.) Possibly this perspective is useful in answering the questions left open above.

(iii) Consider once more the representations (7.10), (7.112)-(7.115) of the pure soliton sG solutions, but now allow arbitrary analytic continuations in $\hat{q}$ and $\hat{\theta}$ (as opposed to continuing to the subsets $\tilde{\hat{\Omega}} \subset C^{2 N}$ with $N_{-}=0,1, \cdots, N$ ). Taking $\hat{\theta}_{1}, \cdots, \hat{\theta}_{N}$ distinct $(\bmod 2 \pi i)$, this yields $\mathrm{sG}$ solutions with non-real values and with singularities, in general. However, the analytic continuations to $\tilde{\hat{\Omega}}$ are not the only ones yielding real-valued real-analytic solutions, as will now be illustrated.

Start from $(\hat{q}, \hat{\theta}) \in \mathbb{R}^{N} \times G_{N}$ and take $\hat{\theta}_{k} \rightarrow \hat{\theta}_{k}+i \pi$ for $k=N_{+}+1, \cdots, N$. Then the resulting matrix $\tilde{A}(0,0)$ equals the Lax matrix (2.70) with $\beta=\mu=1, \tau=\pi / 2$, the real numbers $p_{1}^{+}, \cdots, p_{N_{-}}^{-}, x_{1}^{+}, \cdots, x_{N_{-}}^{-}$being given by $\hat{q}_{1}, \cdots, \hat{q}_{N}, \hat{\theta}_{1}, \cdots, \hat{\theta}_{N}$, resp. Moreover, the space-time dependence of $\tilde{A}(t, y)$ is governed by the Hamiltonians $\tilde{H}^{0} \equiv \operatorname{Tr}\left(A+A^{-1}\right) / 2, \tilde{H}^{1} \equiv \operatorname{tr}\left(A-A^{-1}\right) / 2$, cf. (2.6) with $\mu=1$. Since $\Omega^{b}$ is manifestly left invariant by the space-time flow $(t, y) \mapsto \exp \left(t \tilde{H}^{0}-y \tilde{H}^{1}\right)$, the spectrum of $\tilde{A}(t, y)$ stays in the right half plane. Thus it follows from (7.112) that one obtains a real-valued real-analytic sG solution in this way.

(iv) It is not obvious, though true, that the solutions described in the previous remark are not new. In particular, the solution we have detailed can also be obtained by taking $\hat{q}_{k} \rightarrow-\hat{q}_{k}+i \pi$ for $k=N_{+}+1, \cdots, N$ (which yields a point in $\tilde{\hat{\Omega}}_{0}$, cf. (4.20)-(4.22)). The crux is, that the rhs of (7.113) is left invariant (mod $\pi / 2)$ under the simultaneous continuations $\hat{q}_{j} \rightarrow-\hat{q}_{j}+i \pi, \hat{\theta}_{j} \rightarrow \hat{\theta}_{j}+i \pi$.

This invariance property seems to have escaped attention, so we add a proof, taking $j=1$ to ease the notation. First, rewrite the rhs of (7.113) as

$$
\operatorname{arctg}\left[\frac{S_{1}-S_{3}+S_{5} \cdots}{1-S_{2}+S_{4} \cdots}\right] \equiv \operatorname{arctg} X
$$

Second, rewrite $(7.114)$ as

$$
S_{l}=\sum_{|I|=l} \prod_{\substack{j \in I \\ e_{j}}} \prod_{\substack{j \in I \\ k \notin I}} c_{j k}, \quad e_{j} \neq 0, \quad c_{j k}=c_{k j} \neq 0 .
$$

Third, note the $j=1$ substitutions amount to 


$$
e_{1} \rightarrow-1 / e_{1}, \quad c_{1 k} \rightarrow 1 / c_{1 k}
$$

Fourth, verify that these replacements entail

$$
\begin{gathered}
S_{1}-S_{3}+S_{5} \cdots \rightarrow-\left(1-S_{2}+S_{4} \cdots\right) / C \\
1-S_{2}+S_{4} \cdots \rightarrow\left(S_{1}-S_{3}+S_{5} \cdots\right) / C
\end{gathered}
$$

where

$$
C \equiv e_{1} \prod_{k>1} c_{1 k}
$$

Hence,

$$
\operatorname{arctg} X \rightarrow \operatorname{arctg}(-1 / X)=\operatorname{arctg} X-\pi / 2 \quad(\bmod \pi)
$$

completing the proof.

(v) On $\Omega_{0}^{b}$ the Hamiltonians of remark (iii) are the pullbacks under $\Phi_{0}$ of the Hamiltonians

$$
\begin{aligned}
& \sum_{j=1}^{N_{+}} h\left(\theta_{j}\right) \prod_{k \neq j}\left|\operatorname{cth} \frac{1}{2}\left(q_{j}-q_{k}\right)\right|-\sum_{j=N_{+}+1}^{N} h\left(\theta_{j}\right) \prod_{k \neq j}\left|\operatorname{cth} \frac{1}{2}\left(q_{j}-q_{k}\right)\right|, \\
& h=\mathrm{ch}, \mathrm{sh}, \quad \theta \equiv\left(x^{s}, x^{\bar{s}}\right), \quad q \equiv\left(p^{s}, p^{\bar{s}}\right)
\end{aligned}
$$

on $\hat{\Omega}_{0}^{b}$, cf. (3.57)-(3.60), (4.20)-(4.22), (4.25), (4.26). (Note that the set of $(q, \theta)$ thus obtained may be viewed as $\Omega^{b}$.) From a phase diagram for the reduced $N=2$ case one reads off that the $\tilde{H}^{0}$ and $\tilde{H}^{1}$ flows do not leave $\Omega_{0}^{b}$ invariant: Generically, the trajectories pass through $\Omega_{1}^{b}$, where $\tilde{A} \simeq L$ has non-real spectrum. Correspondingly, the flows generated by the Hamiltonians (7.178) are incurably incomplete on $\hat{\Omega}_{0}^{b}$ for $N=2$.

It is highly plausible that the same incompleteness phenomenon occurs for $N>2$, too. (To prove that it does occur, one needs more information on the geometry of the connected components of $\Omega_{0}^{b}$ vs. those of $\Omega_{l}^{b}, l=1, \cdots, m$.) Note that from a physical point of view the instability is no great surprise, since (7.178) says one is dealing with negative rest mass particles.

All the same, our conclusion is, that sG solutions containing $N_{+}$solitons and $N_{-}$antisolitons can be related not only to $N_{+}$particles and $N_{-}$antiparticles (having the same positive rest mass), but also to $N_{+}$positive rest mass and $N_{-}$negative rest mass particles.

(vi) For distinct points $P_{1}, P_{2}$ in $\Omega^{b}$ one obtains distinct solutions from (7.153) 
and distinct trajectory sets from (7.158), provided that $P_{1}$ and $P_{2}$ belong to $\Omega_{s}^{b}$ and have images $\hat{P}_{1}, \hat{P}_{2}$ in $\hat{\Omega}_{\neq}$under the action-angle map $\Phi$. (Indeed, this follows from Th. 7.3 and its proof; specifically, one can read off $\hat{P}_{i}$ from the $t \rightarrow \infty$ asymptotics.) The natural conjecture is, that the proviso may be omitted, but a proof (or counterexample) has not materialized.

(vii) It should be stressed that the (eventual) pathologies and open questions encountered above all have to do with sets of measure zero. In classical mechanics such sets are among the most interesting ones and should not be ignored. Recall however, that as a rule of thumb measure-zero phenomena are washed away under quantization. In fact, there is mounting evidence that the classical particle-soliton correspondence (which is particularly transparent for the open dense full measure set $\hat{\Omega}_{\neq}$) turns into physical equivalence for the quantum version of the $\tau=\pi / 2 \tilde{\mathrm{I}}_{\mathrm{rel}}$ particle systems and the sine-Gordon quantum field theory, cf. [3] and papers to appear.

Modified KdV. As announced at the beginning of this section, the mKdV case can be handled in much the same way as the $\mathrm{sG}$ case. The analog of the distinct velocity subset (7.118) reads

$$
\begin{aligned}
& \hat{\Omega}_{l \neq}=\left\{\hat{P} \in \hat{\Omega}_{l} \mid \exp 2 p_{1}^{s}, \cdots, \exp 2 p_{k_{+}}^{s}, \exp 2 p_{1}^{\bar{s}}, \cdots, \exp 2 p_{k_{-}}^{\bar{s}}\right. \\
& \left.\quad \exp p_{1} \cos \frac{3}{2} \delta_{1} / \cos \frac{1}{2} \delta_{1}, \cdots, \exp p_{l} \cos \frac{3}{2} \delta_{l} / \cos \frac{1}{2} \delta_{l} \text { distinct }\right\}
\end{aligned}
$$

In this case, the positive numbers $\sigma_{j}$ and distinct numbers $v_{j}$ are obtained by permuting the pairs $\left(\exp p_{1}^{s}, \exp 2 p_{1}^{s}\right), \cdots,\left(\exp \frac{1}{2} p_{1} \cos \frac{1}{2} \delta_{1}, \exp p_{1} \cos \frac{3}{2} \delta_{1} / \cos \frac{1}{2} \delta_{1}\right)$ $\cdots,\left(\exp p_{1}^{\bar{s}}, \exp 2 p_{1}^{\bar{s}}\right), \cdots$. Note the curious fact that $b$ velocities vary over $\boldsymbol{R}$, whereas $s$ and $\bar{s}$ velocities vary over $(0, \infty)$.

To obtain the analog of Th. 7.3, we need the following changes in the formulas (7.119)-(7.134):

$$
\begin{aligned}
& (7.119) \rightarrow(s) v_{\lambda}=\exp 2 p_{i}^{s} \\
& (7.122) \rightarrow f_{i} \equiv t \exp 3 p_{i}^{s}-y \exp p_{i}^{s} \\
& (7.123) \rightarrow(\bar{s}) v_{\lambda}=\exp 2 p_{j}^{\bar{s}} \\
& (7.126) \rightarrow \bar{f}_{j} \equiv t \exp 3 p_{j}^{\bar{s}}-y \exp p_{j}^{\bar{s}}
\end{aligned}
$$


$(7.127) \rightarrow(b) v_{\lambda}=\exp p_{n} \cos \frac{3}{2} \delta_{n} / \cos \frac{1}{2} \delta_{n}$

(7.131) $\rightarrow f_{n}^{+} \equiv t \exp \frac{3}{2} p_{n} \cos \frac{3}{2} \delta_{n}-y \exp \frac{1}{2} p_{n} \cos \frac{1}{2} \delta_{n}$

(7.132) $\rightarrow f_{n}^{-} \equiv-t \exp \frac{3}{2} p_{n} \sin \frac{3}{2} \delta_{n}+y \exp \frac{1}{2} p_{n} \sin \frac{1}{2} \delta_{n}$

Theorem 7.5. With the above changes understood, the assertions of Th. 7.3 hold true.

Proof. Replacing (7.138) by

$$
h(z) \equiv \frac{1}{3} \exp 3 z-\left(v_{\lambda}+s\right) \exp z
$$

and taking $\operatorname{ch} p_{i}^{s} \rightarrow \exp p_{i}^{s}$ in (7.140), the proof of Th. 7.3 applies verbatim; the monotonicity claim will be proved below.

Following the reasoning of the $\mathrm{sG}$ case, the second item of the program can be disposed of when (7.147) is replaced by

$$
A(t, y, P) \equiv A(P) \exp \left[t L(P)^{3}-y L(P)\right]
$$

The third one amounts to the following analog of Th. 7.4.

Theorem 7.6. The function $v \equiv \partial_{y} \psi(t, y)$ corresponding to $P \in \Omega^{b}$ via (7.135) is a real-valued real-analytic solution to the modified KdV equation (7.2), satisfying $v \in S(R)$. The solution yields a reflectionless potential in the sense of the IST.

Proof. Replacing (7.154) and (7.155) by

$$
\begin{gathered}
M(y, P) \equiv \exp [-y L(P)]=\frac{1}{2 \pi i} \int_{\Gamma} \exp (-y z) R(z, L(P)) d z \\
\sigma_{-} \equiv \min _{\lambda \in \sigma(L(P))} \operatorname{Re} \lambda
\end{gathered}
$$


the reasoning in the poof of Th. 7.4 can be followed.

Finally, we come to the topic of space-time trajectories. Again, the trajectory set $T_{p}$ defined via (7.158) cannot be equal to $\boldsymbol{R}^{2}$, since $A(t, y, P) \rightarrow 0$ as $y \rightarrow \infty$. More generally, setting

$$
v_{ \pm} \equiv \min _{\lambda \in \sigma(L(P))} \frac{\operatorname{Re} \lambda^{3}}{\operatorname{Re} \lambda}
$$

the discussion below (7.159) applies, provided the adjective 'timelike' and the remark on light cones are omitted. In particular, it follows that (7.160) holds true for $u=0$.

Replacing (7.163) by

$$
\partial_{y} x_{k}^{\delta}(t, y)=\left[-\exp \left(p_{k}^{\delta}\right) V_{k}^{\delta}\right](t, y)<0, \quad(t, y) \in \boldsymbol{R}^{2} \backslash C_{p}
$$

it now follows as before that the functions $y \mapsto x_{k}^{\delta}\left(t_{0}, y\right)$ are strictly decreasing. Then (7.160) with $u=0$ suffices to conclude that (7.161) yields unique solutions $y_{k}^{\delta}(t)$, and as a consequence (7.162) holds true.

We leave a study of the local structure of the trajectories to the interested reader. To conclude our account of the $\mathrm{mKdV}$ case, we observe that, for points in the open dense full measure distinct velocity subset of $\Omega^{b}$, the trajectory asymptotics for $t \rightarrow \pm \infty$ agrees with a plot of the solution, in the same sense as for the $\mathrm{sG}$ trajectories.

\section{Appendix A. Pseudo-self-adjoint and Pseudo-unitary Matrices}

Throughout this appendix $\mathscr{J}$ denotes an operator on an $\mathrm{N}$-dimensional (complex) Hilbert space $\mathscr{H}$, which is both unitary and self-adjoint. Thus $\mathscr{H}$ and $\mathscr{J}$ can be written

$$
\begin{aligned}
& \mathscr{H}=\mathscr{H}_{+} \oplus \mathscr{H}_{-}, \quad \mathscr{H}_{\delta}=P_{\delta} \mathscr{H}, \quad \delta=+,- \\
& \mathscr{J}=P_{+}-P_{-},
\end{aligned}
$$

where $P_{+}, P_{-}$are uniquely determined orthogonal projections. We denote $\operatorname{dim} \mathscr{H}_{\delta}$ by $N_{\delta}$. By definition, an operator $L$ on $\mathscr{H}$ is pseudo-self-adjoint w.r.t. $\mathscr{J}$ (abbreviated $\mathscr{J}$-s.a.) if it satisfies 


$$
L^{*}=\mathscr{J} L \mathscr{J}
$$

(where * denotes the Hilbert space adjoint), and an operator $U$ is pseudo-unitary w.r.t. $\mathscr{J}$ (abbreviated $\mathscr{J}$-unitary) if it satisfies

$$
U^{*} \mathscr{J} U=\mathscr{J} .
$$

In this appendix we collect some material concerning $\mathscr{J}$-s.a. and $\mathscr{J}$-unitary operators. In essence, all of what follows has been known for many decades, cf. e.g. [21] and references given there. However, we present simple proofs for three results we have occasion to use, since we have not found a reference from which a proof can be gleaned without undue effort.

We begin with a few Facts that are easily verified.

(1) $\mathscr{J}$-unitaries form a subgroup of $G L(\mathscr{H})$;

(2) If $L$ is $\mathscr{J}$-s.a. and $U$ is $\mathscr{J}$-unitary, then $U^{-1} L U$ is $\mathscr{J}$-s.a.;

(3) $L$ is $\mathscr{J}$-s.a. if and only if $L \mathscr{J}$ is self-adjoint;

(4) The spectrum of a $\mathscr{J}$-s.a. operator is symmetric w.r.t. the real axis;

(5) If $\varphi_{1}, \varphi_{2}$ are eigenvectors of a $\mathscr{J}$-s.a. operator with eigenvalues $\lambda_{1}, \lambda_{2}$ such that $\lambda_{2} \neq \bar{\lambda}_{1}$, then $\varphi_{1}$ and $\varphi_{2}$ are $\mathscr{J}$-orthogonal (i.e., $\left(\varphi_{1}, \mathscr{J} \varphi_{2}\right)=0$ );

(6) If $\varphi \in \mathscr{H}_{\delta}$ and $L$ is $\mathscr{J}$-s.a., then $(\varphi, L \varphi) \in \boldsymbol{R}$;

(7) If $S \in G L(\mathscr{H})$, then $S^{*} \mathscr{J} S$ has $N_{+}$positive and $N_{-}$negative eigenvalues ('Sylvester').

We continue with some results whose proofs are less immediate.

Proposition A1. Suppose $A$ is $\mathscr{J}$-s.a. and such that $B \equiv A \mathscr{J}$ is positive. Then $A$ is diagonalizable and has $N_{+} / N_{-}$eigenvalues in $\left[C_{-}, C_{+}\right] /\left[-C_{+},-C_{-}\right]$, where $C_{+} / C_{-}$denotes a maximal/minimal eigenvalue of $B$. Moreover, any non-zero vector $\varphi_{+} / \varphi_{-}$in the positive/negative spectral subspace of A satisfies $\delta\left(\varphi_{\delta}, \mathscr{J} \varphi_{\delta}\right)>0$, $\delta=+,-$.

Proof. Since $A$ equals $B \mathscr{J}, A$ is similar to the self-adjoint operator $C \equiv B^{1 / 2} \mathscr{J} B^{1 / 2}$ and hence diagonalizable. Also, $C$ has $N_{+}$positive and $N_{-}$ negative eigenvalues by virtue of Fact (7). These lie in the intervals specified above, since $\|C\| \leqslant\|B\|=C_{+}$and $\left\|C^{-1}\right\| \leqslant\left\|B^{-1}\right\|=1 / C_{-}$. Finally, if $A \varphi=\lambda \varphi$ with $\varphi \neq 0$, then $\lambda(\varphi, \mathscr{J} \varphi)=(\varphi, \mathscr{J} B \mathscr{J} \varphi)>0$, from with the last assertion follows.

Proposition A2. Suppose $L$ is $\mathscr{J}$-s.a. and suppose a $\mathscr{J}$-unitary $U$ exists such that $U^{-1} L U$ commutes with $\mathscr{J}$. Then $L$ is diagonalizable and has real spectrum. 
Proof. The assumptions entail

$$
\left(U^{-1} L U\right)^{*}=\left(\mathscr{J} U^{-1} \mathscr{J}\right)(\mathscr{J} L \mathscr{J})(\mathscr{J} U \mathscr{J})=\mathscr{J}\left(U^{-1} L U\right) \mathscr{J}=U^{-1} L U
$$

so that $L$ is similar to a self-adjoint operator.

From now on we identify $\mathscr{H}$ with $C^{N}$ equipped with its standard scalar product, and we take

$$
\mathscr{J} \equiv\left[\begin{array}{cc}
\mathbf{1}_{N_{+}} & 0 \\
0 & -\mathbf{1}_{N_{-}}
\end{array}\right], \quad N_{+}, N_{-} \geqslant 1
$$

The third and last proposition is concerned with a $\mathscr{J}$-s.a. operator $L$ whose spectrum is simple. By virtue of Fact (4) this implies

$$
\sigma(L)=\left\{\boldsymbol{r}_{1}, \cdots, r_{k}, \rho_{1} \pm i \sigma_{1}, \cdots, \rho_{l} \pm i \sigma_{l}\right\}, \quad r_{i}, \rho_{j} \in \boldsymbol{R}, \sigma_{j} \in(0, \infty), k+2 l=N
$$

Proposition A3. In (A6) one has

$$
k \geqslant\left|N_{+}-N_{-}\right|, \quad l \leqslant \min \left(N_{+}, N_{-}\right)
$$

There exists a $\mathscr{J}$-unitary $U$ such that

$$
U^{-1} L U=\left[\begin{array}{cccc}
D_{+} & & & 0 \\
& R & \Sigma & \\
& \Sigma & R & \\
& & & D_{-}
\end{array}\right]
$$

Here, one has

$$
\begin{aligned}
& D_{+} \equiv \operatorname{diag}\left(p_{1}, \cdots, p_{k_{+}}\right), \quad D_{-} \equiv \operatorname{diag}\left(n_{1}, \cdots, n_{k_{-}}\right) \\
& R \equiv \operatorname{diag}\left(\rho_{1}, \cdots, \rho_{l}\right), \quad \Sigma \equiv \operatorname{diag}\left(i \sigma_{1}, \cdots, i \sigma_{l}\right)
\end{aligned}
$$

and

$$
\begin{gathered}
k_{+}+k_{-}=k, \quad k_{+}+l=N_{+}, \quad l+k_{-}=N_{-} \\
\left\{p_{1}, \cdots, p_{k_{+}}\right\} \cup\left\{n_{1}, \cdots, n_{k_{-}}\right\}=\left\{r_{1}, \cdots, r_{k}\right\} .
\end{gathered}
$$

Proof. Let $\varphi_{i}$ and $\psi_{j}^{\delta}$ denote eigenvectors corresponding to the eigenvalues 
$r_{i}$ and $\rho_{j}+i \delta \sigma_{j}$, resp., and set

$$
S \equiv \operatorname{Col}\left(\varphi_{1}, \cdots, \varphi_{k}, \psi_{1}^{+}, \psi_{1}^{-}, \cdots, \psi_{l}^{+}, \psi_{l}^{-}\right) .
$$

Since $\sigma(L)$ is nondegenerate, the above Fact (5) implies

$$
S^{*} \mathscr{J} S=\operatorname{diag}\left(\left(\varphi_{1}, \mathscr{J} \varphi_{1}\right), \cdots,\left(\varphi_{k}, \mathscr{J} \varphi_{k}\right), M_{1}, \cdots, M_{l}\right)
$$

where

$$
M_{j} \equiv\left[\begin{array}{cc}
0 & \left(\psi_{j}^{+}, \mathscr{J} \psi_{j}^{-}\right) \\
\left(\psi_{j}^{-}, \mathscr{J} \psi_{j}^{+}\right) & 0
\end{array}\right] .
$$

Moreover, since $S$ is regular, one has $\left(\varphi_{i}, \mathscr{J} \varphi_{i}\right),\left(\psi_{j}^{\delta}, \mathscr{J} \psi_{j}^{-\delta}\right) \neq 0$. Via a scaling we can therefore arrange

$$
\begin{aligned}
& \left(\varphi_{i}, \mathscr{J} \varphi_{i}\right)=s_{i}, \quad s_{i} \in\{1,-1\}, \quad i=1, \cdots, k \\
& \left(\psi_{j}^{\delta}, \mathscr{J} \psi_{j}^{-\delta}\right)=\frac{1}{2}, \quad j=1, \cdots, l, \quad \delta=+,-
\end{aligned}
$$

Next, we introduce

$$
T \equiv \operatorname{Col}\left(\varphi_{1}, \cdots, \varphi_{k}, \chi_{1}^{+}, \chi_{1}^{-}, \cdots, \chi_{l}^{+}, \chi_{l}^{-}\right)
$$

where

$$
\chi_{j}^{\delta} \equiv \psi_{j}^{+}+\delta \psi_{j}^{-}, \quad j=1, \cdots, l, \quad \delta=+,-
$$

Then (A16) implies

$$
T^{*} \mathscr{J} T=\operatorname{diag}\left(s_{1}, \cdots, s_{k}, 1,-1, \cdots, 1,-1\right) .
$$

By virtue of Fact (7) the sign $+(-)$ must occur $N_{+}\left(N_{-}\right)$times at the $r h s$, so that (A7) follows.

Now we partition the real eigenvalues as in (A12), such that the eigenvectors $\varphi_{1}^{+}, \cdots, \varphi_{k_{+}}^{+}$and $\varphi_{1}^{-}, \cdots, \varphi_{k_{-}}^{-}$corresponding to $p_{1}, \cdots, p_{k_{+}}$and $n_{1}, \cdots, n_{k_{-}}$, resp., satisfy $\left(\varphi_{j}^{ \pm}, \mathscr{J} \varphi_{j}^{ \pm}\right)= \pm 1$. Then (A11) follows from the above sign count, and setting

$$
U \equiv \operatorname{Col}\left(\varphi_{1}^{+}, \cdots, \varphi_{k_{+}}^{+}, \chi_{1}^{+}, \cdots, \chi_{l}^{+}, \chi_{1}^{-}, \cdots, \chi_{l}^{-}, \varphi_{1}^{-}, \cdots, \varphi_{k_{-}}^{-}\right)
$$

it follows that $U$ is $\mathscr{J}$-unitary. Moreover, (A8) is readily verified, using 


$$
U^{-1}=\mathscr{J} U^{*} \mathscr{J}, \quad L \chi_{j}^{\delta}=\rho_{j} \chi_{j}^{\delta}+i \sigma_{j} \chi_{j}^{-\delta}
$$

cf. (A18).

Note that it follows from Prop. A2 that $L$ cannot be diagonalized by a $\mathscr{J}$-unitary whenever $l>0$. The normal form derived here may be viewed as 'next best'; it appears to date back to the $19^{\text {th }}$ century. Note it implies that for $\mathscr{J}$-s.a. operators with simple spectrum any eigenvector $\varphi$ corresponding to an eigenvalue $r \in \boldsymbol{R}$ satisfies either $(\varphi, \mathscr{J} \varphi)>0$ or $(\varphi, \mathscr{J} \varphi)<0$. We shall refer to $r$ as a positive/negative signature eigenvalue in the first/second case.

\section{Appendix B. The Cauchy Matrix Revisited}

In this paper it is convenient to work with the Cauchy matrix

$$
C(\beta, \mu, g ; p, q)_{j k} \equiv \exp \left(-\frac{1}{2} \beta p_{j}\right) \frac{\operatorname{sh}\left(\frac{1}{2} i \beta \mu g\right)}{\operatorname{sh} \frac{1}{2} \beta\left(p_{j}-q_{k}+i \mu g\right)} \exp \left(-\frac{1}{2} \beta q_{k}\right), \quad j, k=1, \cdots, M
$$

where the $r h s$ is defined in the obvious way when $\beta=0$. Compared to our previous paper $\mathrm{I}$, this amounts to adding exponentials, a reparametrization and a slight generalization: Here, the case $q \neq p$ naturally arises. Setting

$$
x_{j} \equiv \exp \left[\beta\left(p_{j}+\frac{1}{2} i \mu g\right)\right], \quad y_{k} \equiv \exp \left[\beta\left(q_{k}-\frac{1}{2} i \mu g\right)\right]
$$

in Cauchy's identity

$$
\left|\left\lceil\frac{1}{x_{j}-y_{k}}\right]\right|=\prod_{j} \frac{1}{x_{j}-y_{j}} \prod_{j<k} \frac{\left(x_{j}-x_{k}\right)\left(y_{j}-y_{k}\right)}{\left(x_{j}-y_{k}\right)\left(y_{j}-x_{k}\right)}
$$

one obtains

$$
|C|=\exp \left[-\frac{1}{2} \beta \sum_{j}\left(p_{j}+q_{j}\right)\right] \prod_{j} \frac{\operatorname{sh}\left(\frac{1}{2} i \beta \mu g\right)}{\operatorname{sh} \frac{1}{2} \beta\left(p_{j}-q_{j}+i \mu g\right)}
$$




$$
\prod_{j<k} \frac{\operatorname{sh} \frac{1}{2} \beta\left(p_{j}-p_{k}\right) \operatorname{sh} \frac{1}{2} \beta\left(q_{j}-q_{k}\right)}{\operatorname{sh} \frac{1}{2} \beta\left(p_{j}-q_{k}+i \mu g\right) \operatorname{sh} \frac{1}{2} \beta\left(q_{j}-p_{k}-i \mu g\right)}
$$

This entails a positivity property that plays a crucial role in this paper.

Lemma B1. Let $\mu, g>0, \beta \in[0,2 \pi / \mu g)$ and let $p_{j}-p_{k} \neq 0, j \neq k,\left|\operatorname{Im} p_{j}\right|<\mu g / 2$, $j, k=1, \cdots, M$. Then $C(\beta, \mu, g ; p, p)$ is positive.

Proof. The assumptions entail not only that the matrix $C \equiv C(\beta, \mu, g ; p, \bar{p})$ is self-adjoint, but also that $C$ has positive principal minors; indeed, the latter property can be read off from (B4). Thus, the polynomial $\left|\lambda \mathbf{1}_{M}+C\right|$ has positive coefficients, so $\sigma(C) \subset(0, \infty)$.

We continue with an observation that will be exploited in Chapter 6: Up to left and right multiplication by diagonal matrices, the inverse and the transpose of $C$ are equal. This fact also plays a key role in the treatment of Sutherland type systems [22].

Lemma B2. Let $(\beta, \mu, g, p, q) \in C^{3+2 M}$ be such that $C \equiv C(\beta, \mu, g ; p, q)$ belongs to $G L(M, C)$. Then one has

$$
C^{-1 t}=\operatorname{diag}\left(l_{1}, \cdots, l_{M}\right) C \operatorname{diag}\left(r_{1}, \cdots, r_{M}\right)
$$

where

$$
\begin{gathered}
l_{j} \equiv \frac{\exp \left[\frac{1}{2} \beta\left(p_{j}-q_{j}\right)\right]}{C_{j j}} \prod_{n \neq j} \frac{\operatorname{sh} \frac{1}{2} \beta\left(p_{j}-q_{n}+i \mu g\right)}{\operatorname{sh} \frac{1}{2} \beta\left(p_{j}-p_{n}\right)} \\
r_{j} \equiv \frac{\exp \left[\frac{1}{2} \beta\left(q_{j}-p_{j}\right)\right]}{C_{j j}} \prod_{n \neq j} \frac{\operatorname{sh} \frac{1}{2} \beta\left(q_{j}-p_{n}-i \mu g\right)}{\operatorname{sh} \frac{1}{2} \beta\left(q_{j}-q_{n}\right)} .
\end{gathered}
$$


Proof. Setting

$$
f(x) \equiv \operatorname{sh} \frac{1}{2} \beta x / \operatorname{sh} \frac{1}{2} i \beta \mu g
$$

and using (B1) we may rewrite (B4) as

$$
|C|=\exp \left[\frac{1}{2} \beta(M-1) \sum_{i}\left(p_{i}+q_{i}\right)\right] \prod_{i, j} C_{i j} \prod_{i<j} f\left(p_{i}-p_{j}\right) f\left(q_{j}-q_{i}\right)
$$

From this we obtain

$$
\begin{aligned}
\left(C^{-1 t}\right)_{k l}= & \left(\text { cofactor of } C_{k l}\right) /|C| \\
= & (-)^{k+1} \exp \left[\frac{1}{2} \beta(M-2)\left(\sum_{i \neq k} p_{i}+\sum_{i \neq l} q_{i}\right)-\frac{1}{2} \beta(M-1)\left(\sum_{i} p_{i}+\sum_{i} q_{i}\right)\right] \\
& \cdot\left[\frac{1}{C_{k l}} \prod_{i} C_{k i} C_{i l} \prod_{i \neq k} \operatorname{sign}(i-k) f\left(p_{k}-p_{i}\right) \prod_{i \neq l} \operatorname{sign}(i-l) f\left(q_{i}-q_{l}\right)\right]^{-1} \\
= & \exp \left[-\frac{1}{2} \beta\left(\sum_{i \neq k} p_{i}+\sum_{i \neq l} q_{i}\right)-\frac{1}{2} \beta(M-1)\left(p_{k}+q_{l}\right)\right] \\
& \cdot\left[C_{k k} \prod_{i \neq k} C_{k i} f\left(p_{k}-p_{i}\right)\right]^{-1} C_{k l}\left[C_{l l} \prod_{i \neq l} C_{i l} f\left(q_{i}-q_{l}\right)\right]^{-1} .
\end{aligned}
$$

Using (B1) once more, this yields (B5) -(B7).

\section{Appendix C. Spectral Asymptotics Revisited}

Partition the integers $\{1, \cdots, N\}$ into $K$ subsets $I_{1}, \cdots, I_{K}$ by setting

$$
I_{\rho} \equiv\left\{n_{\rho-1}+1, \cdots, n_{\rho}\right\}, \quad \rho=1, \cdots, K
$$

where

$$
0 \equiv n_{0}<n_{1}<\cdots<n_{K} \equiv N, \quad 1<K \leqslant N
$$

and let $\mathscr{J}_{\rho}(t)$ be $\left|I_{\rho}\right| \times\left|I_{\rho}\right|$ matrices satisfying 


$$
\mathscr{J}_{\rho}(t) \in U\left(\left|I_{\rho}\right|\right), \quad \mathscr{J}_{\rho}(t)^{*}=\mathscr{J}_{\rho}(t), \quad \forall t \in R, \quad \forall \rho \in\{1, \cdots, K\}
$$

This appendix is concerned with the spectral asymptotics for $|t| \rightarrow \infty$ of $N \times N$ matrices of the form

$$
E(t)=M \operatorname{diag}\left(\exp \left(t d_{1}\right) \mathscr{J}_{1}(t), \cdots, \exp \left(t d_{K}\right) \mathscr{J}_{K}(t)\right)
$$

where

$$
\begin{aligned}
& M>0 \\
& d_{K}<\cdots<d_{1}, \quad 1<K \leqslant N .
\end{aligned}
$$

Introducing

$$
C_{+} \equiv\|M\|, \quad C_{-} \equiv\left\|M^{-1}\right\|^{-1},
$$

one clearly has $\sigma(M) \subset\left[C_{-}, C_{+}\right]$. Setting

$$
I_{\rho}^{+} \equiv\left\{1, \cdots, n_{\rho-1}\right\}, \quad I_{\rho}^{-} \equiv\left\{n_{\rho}+1, \cdots, N\right\}, \quad \rho=1, \cdots, K
$$

we denote by $M_{\rho}^{+} / M_{\rho}^{-}$the matrices obtained from $M$ by discarding the rows and columns whose indices belong to $I_{\rho}^{-} / I_{\rho}^{+}$. Then $\sigma\left(M_{\rho}^{\delta}\right) \subset\left[C_{-}, C_{+}\right]$, so that $M_{\rho}^{\delta}$ is invertible and positive. We denote by $\left(\left(M_{\rho}^{\delta}\right)^{-1}\right)_{\rho}$ the $\left|I_{\rho}\right| \times\left|I_{\rho}\right|$ matrix obtained from $\left(M_{\rho}^{\delta}\right)^{-1}$ by retaining only the $\left|I_{\rho}\right|$ last/first rows and columns when $\delta$ equals $+/$. Setting now

$$
m_{\rho}^{\delta} \equiv\left(\left(\left(M_{\rho}^{\delta}\right)^{-1}\right)_{\rho}\right)^{-1}, \quad \rho=1, \cdots, K, \quad \delta=+,-
$$

it follows that $m_{\rho}^{ \pm}$are positive $\left|I_{\rho}\right| \times\left|I_{\rho}\right|$ matrices with spectrum in $\left[C_{-}, C_{+}\right]$.

In the special case $K=N, \mathscr{J}_{\rho}(t)=1$, the asymptotics of $\sigma(E(t))$ follows from Theorem $\mathrm{A} 2$ in I. Returning to the general case, we introduce the cluster matrices

$$
c_{\rho}^{\delta}(t) \equiv m_{\rho}^{\delta} \mathscr{J}_{\rho}(t), \quad \rho=1, \cdots, K, \quad \delta=+,-
$$

By virtue of Prop. A1 these matrices are diagonalizable and have real eigenvalues $c_{\rho 1}^{\delta}(t) \geqslant \cdots \geqslant c_{\rho\left|I_{\rho}\right|}^{\delta}(t)$ satisfying

$$
c_{\rho j}^{\delta}(t) \in\left[-C_{+},-C_{-}\right] \cup\left[C_{-}, C_{+}\right], \quad j=1, \cdots,\left|I_{\rho}\right|, \quad \rho=1, \cdots, K, \quad \delta=+,-.
$$

Likewise, $E(t)$ is similar to a self-adjoint and invertible matrix and hence has real and non-zero eigenvalues $\alpha_{1}(t), \cdots, \alpha_{N}(t)$. Setting 


$$
r_{1} \equiv d_{1}-d_{2}, r_{K} \equiv d_{K-1}-d_{K}, r_{\rho} \equiv \min \left(d_{\rho-1}-d_{\rho}, d_{\rho}-d_{\rho+1}\right), \rho=2, \cdots, K-1
$$

we are prepared for the following theorem, which reduces the spectral asymptotics of $E(t)$ for $t \rightarrow \pm \infty$ to that of the cluster matrices $c_{\rho}^{ \pm}(t)$.

Theorem C1. Fix $\rho \in\{1, \cdots, K\}$ and define $T_{\rho}>0$ by

$$
\exp \left(-T_{\rho} r_{\rho}\right)=C_{-} / 3 C_{+} .
$$

Choosing $\pm t>T_{\rho}$, the matrix E(t) has $\left|I_{\rho}\right|$ eigenvalues $\alpha_{\rho 1}^{ \pm}(t) \geqslant \cdots \geqslant \alpha_{\rho\left|I_{\rho}\right|}^{ \pm}(t)$ obeying

$$
\left|\alpha_{\rho j}^{ \pm}(t)\right| \in\left(2 C_{-} / 3,2 C_{+}\right) \exp \left(t d_{\rho}\right), \quad j=1, \cdots,\left|I_{\rho}\right|
$$

$\left|I_{\rho}^{\mp}\right|$ eigenvalues with moduli $\leqslant C_{+} \exp \left(t d_{\rho \pm 1}\right)<\frac{1}{3} C_{-} \exp \left(t d_{\rho}\right)$ and $\left|I_{\rho}^{ \pm}\right|$eigenvalues with moduli $\geqslant C_{-} \exp \left(t d_{\rho \mp 1}\right)-C_{+} \exp \left(t d_{\rho \pm 1}\right)>\frac{8}{3} C_{+} \exp \left(t d_{\rho}\right)$. Moreover, one has

$$
\begin{array}{r}
\left|\exp \left(-t d_{\rho}\right) \alpha_{\rho j}^{\delta}(t)-c_{\rho j}^{\delta}(t)\right|<5 C_{+}^{2} / 2 C_{-} \exp \left(|t| r_{\rho}\right), \\
j=1, \cdots,\left|I_{\rho}\right|, \quad \delta t>T_{\rho}, \quad \delta=+,-
\end{array}
$$

Proof. Using the reversal permutation matrix, the case $-t>T_{\rho}$ is easily reduced to the case $t>T_{\rho}$. Therefore, we take $t>0$ from now on. Consider the auxiliary matrix

$$
A(t) \equiv \exp \left(-t d_{\rho}\right) E(t)
$$

Setting

$$
e_{\tau}(t) \equiv \exp \left(t d_{\tau}-t d_{\rho}\right), \quad \tau=1, \cdots, K
$$

it satisfies (recall $\sim$ denotes similarity)

$$
\begin{aligned}
A(t) & \sim M^{1 / 2} \operatorname{diag}\left(e_{1}(t) \mathscr{J}_{1}(t), \cdots, \mathscr{J}_{\rho}(t), 0, \cdots, 0\right) M^{1 / 2} \\
& +M^{1 / 2} \operatorname{diag}\left(0, \cdots, 0, e_{\rho+1}(t) \mathscr{J}_{\rho+1}(t), \cdots, e_{K}(t) \mathscr{J}_{K}(t)\right) M^{1 / 2} \equiv B(t)+S(t) .
\end{aligned}
$$

In order to study the spectrum of the 'big' (for $t \rightarrow \infty$ ) matrix $B(t)$ we introduce the $n_{\rho} \times n_{\rho}$ matrix

$$
F(t) \equiv \operatorname{diag}\left(e_{1}(t)^{-1} \mathscr{J}_{1}(t), \cdots, \mathscr{J}_{\rho}(t)\right)\left(M_{\rho}^{+}\right)^{-1}
$$


and observe that

$$
B(t) \sim\left[\begin{array}{cc}
F(t)^{-1} & 0 \\
* & 0
\end{array}\right] .
$$

Therefore, $B(t)$ has $N-n_{\rho}$ zero eigenvalues, the remaining eigenvalues being those of $F(t)^{-1}$. To obtain information on the latter, we first note

$$
\begin{aligned}
& F(t) \sim\left(M_{\rho}^{+}\right)^{-1 / 2} \operatorname{diag}\left(0, \cdots, 0, \mathscr{J}_{\rho}(t)\right)\left(M_{\rho}^{+}\right)^{-1 / 2} \\
& +\left(M_{\rho}^{+}\right)^{-1 / 2} \operatorname{diag}\left(e_{1}(t)^{-1} \mathscr{J}_{1}(t), \cdots, e_{\rho-1}(t)^{-1} \mathscr{J}_{\rho-1}(t), 0\right)\left(M_{\rho}^{+}\right)^{-1 / 2} \equiv H(t)+V(t)
\end{aligned}
$$

and then observe

$$
H(t) \sim\left[\begin{array}{cc}
0 & * \\
0 & \left(m_{\rho}^{+}\right)^{-1} \mathscr{J}_{\rho}(t)
\end{array}\right] .
$$

Therefore, $H(t)$ has $n_{\rho-1}$ zero eigenvalues, the remaining $\left|I_{\rho}\right|$ eigenvalues being those of $\left(m_{\rho}^{+}\right)^{-1} \mathscr{J}_{\rho}(t)$. But the latter matrix is similar to the inverse of the cluster matrix $c_{\rho}^{+}(t)$, so that

$$
\sigma(H(t)) \backslash\{0\}=\left\{c_{\rho 1}^{+}(t)^{-1}, \cdots, c_{\rho\left|I_{\rho}\right|}^{+}(t)^{-1}\right\} .
$$

Having disposed of the algebra, we are prepared to handle the analysis. First, we observe that the definitions (C19) and (C21) of $F(t)$ and $V(t)$ entail

$$
\begin{gathered}
\|F(t)\| \leqslant 1 / C_{-} \\
\|V(t)\| \leqslant 1 / C_{-} e_{\rho-1}(t)
\end{gathered}
$$

resp. Choosing $T^{-}$such that

$$
e_{\rho-1}\left(T^{-}\right)^{-1}=C_{-} / 3 C_{+}
$$

the latter estimate implies

$$
\|V(t)\|<1 / 3 C_{+}, \quad \forall t>T^{-} .
$$

Now from $(\mathrm{C} 11)$ we have

$$
\left|c_{\rho j}^{+}(t)^{-1}\right| \geqslant 1 / C_{+}, \quad j=1, \cdots,\left|I_{\rho}\right| .
$$


Combining this with (C23), (C21) and (C27), and recalling the bounds (C24) and (C25), we deduce that for any $t>T^{-}$the matrix $F(t)$ has $n_{\rho-1}$ eigenvalues with modulus $\leqslant 1 / C_{-} e_{\rho-1}(t)<1 / 3 C_{+}$, the remaining ones having modulus in the interval $\left(2 / 3 C_{+}, 1 / C_{-}\right]$. Moreover, denoting the latter by $\varphi_{\rho j}(t)$ and requiring $\varphi_{\rho 1}(t)^{-1} \geqslant \cdots \geqslant \varphi_{\rho\left|I_{\rho}\right|}(t)^{-1}$, we may combine (C21), (C23) and (C25) with the min-max principle to infer

$$
\left|\varphi_{\rho j}(t)-c_{\rho j}^{+}(t)^{-1}\right| \leqslant 1 / C_{-} e_{\rho-1}(t), \quad \forall t>T^{-} .
$$

Clearly, these bounds entail

$$
\begin{aligned}
\left|\varphi_{\rho j}(t)^{-1}-c_{\rho j}^{+}(t)\right| & \leqslant\left|\varphi_{\rho j}(t)^{-1} c_{\rho j}^{+}(t)\right| / C_{-} e_{\rho-1}(t) \\
& <3 C_{+}^{2} / 2 C_{-} e_{\rho-1}(t), \quad \forall t>T^{-} .
\end{aligned}
$$

Recalling now (C20), we deduce that for any $t>T^{-}$the matrix $B(t)$ has $\left|I_{\rho}^{-}\right|$ zero eigenvalues, $\left|I_{\rho}^{+}\right|$eigenvalues with moduli $\geqslant C_{-} e_{\rho-1}(t)>3 C_{+}$, and $\left|I_{\rho}\right|$ eigenvalues $\varphi_{\rho j}(t)^{-1}$ having moduli in $\left[C_{-}, 3 C_{+} / 2\right)$ and obeying (C30). Next, we consider the matrix $S(t)$ defined by (C18). It satisfies

$$
\|S(t)\| \leqslant C_{+} e_{\rho+1}(t)
$$

so that we obtain

$$
\|S(t)\|<C_{-} / 3, \quad \forall t>T^{+}
$$

provided $T^{+}$is defined by

$$
e_{\rho+1}\left(T^{+}\right)=C_{-} / 3 C_{+} .
$$

Since we have (cf. (C13))

$$
T_{\rho}=\max \left(T^{-}, T^{+}\right),
$$

it follows that for any $t>T_{\rho}$ the matrix $A(t)$ has $\left|I_{\rho}^{-}\right|$eigenvalues with moduli $\leqslant C_{+} e_{\rho+1}(t)<C_{-} / 3,\left|I_{\rho}^{+}\right|$eigenvalues with moduli $\geqslant C_{-} e_{\rho-1}(t)-C_{+} e_{\rho+1}(t)>$ $8 C_{+} / 3$, and $\left|I_{\rho}\right|$ eigenvalues with moduli in $\left(2 C_{-} / 3,2 C_{+}\right)$. Moreover, denoting the latter by $\gamma_{\rho j}(t)$ and requiring $\gamma_{\rho 1}(t) \geqslant \cdots \geqslant \gamma_{\rho\left|I_{\rho}\right|}(t)$, a second application of the min-max principle yields 


$$
\begin{array}{rlrl}
\left|\gamma_{\rho j}(t)-c_{\rho j}^{+}(t)\right| & \leqslant\left|\gamma_{\rho j}(t)-\varphi_{\rho j}(t)^{-1}\right|+\left|\varphi_{\rho j}(t)^{-1}-c_{\rho j}^{+}(t)\right| \\
& <C_{+} e_{\rho+1}(t)+3 C_{+}^{2} / 2 C_{-} e_{\rho-1}(t) & \\
& <5 C_{+}^{2} / 2 C_{-} \exp \left(t r_{\rho}\right) \quad \forall t>T_{\rho} .
\end{array}
$$

Recalling (C16), the theorem now follows.

The result just obtained can be rephrased as follows. For long times the matrix $E(t)$ has $K$ disjoint clusters of eigenvalues associated with the distinct numbers $d_{1}, \cdots, d_{K}$ via (C15). The cluster corresponding to $d_{\rho}$ is isolated for $T \leqslant-T_{\rho}$ and $T \geqslant T_{\rho}$, but will collide with the remaining eigenvalues for $|t|<T_{\rho}$, in general. (For instance, collisions clearly occur for diagonal $M$.)

Notice that the upper bound $2 T_{\rho}$ on the collision period is not optimal, the reason being that all collisions may take place long before or long after $t=0$. To take this into account, consider the matrix

$$
M(t) \equiv M \operatorname{diag}\left(\exp \left(t d_{1}\right) \mathbf{1}_{\left|I_{1}\right|}, \cdots, \exp \left(t d_{K}\right) \mathbf{1}_{\left|I_{\mathbf{K}}\right|} .\right.
$$

Denoting the maximal and minimal eigenvalues of $M(t)$ by $C_{+}(t)$ and $C_{-}(t)$, resp. (so that $C_{\delta}(0)=C_{\delta}$, cf. (C7)) we may use Theorem $\mathrm{C} 1$ to infer $C_{-}(t) / C_{+}(t) \rightarrow 0$ for $|t| \rightarrow \infty$. Hence the function $t \mapsto C_{-}(t) / 3 C_{+}(t)$ attains its supremum $s \in\left(0, \frac{1}{3}\right]$ on a compact set $B$. If $0 \notin B$, then the upper bound $2 T_{\rho}$ can be decreased to $-2 r_{\rho}^{-1} \ln s$ by changing the time origin to some $t \in B$, cf. (C13).

\section{References}

[1] Ruijsenaars, S.N.M., Action-angle maps and scattering theory for some finite-dimensional integrable systems. I. The pure soliton case, Commun. Math. Phys., 115 (1988), 127-165.

[2] - Relativistic Calogero-Moser systems and solitons, In: Topics in soliton theory and exactly solvable nonlinear equations, Ablowitz, M., Fuchssteiner, B., Kruskal, M. (eds.), 182-190, World Scientific, Singapore, 1987.

[3] - Finite-dimensional soliton systems, In: Integrable and superintegrable systems, Kupershmidt, B. (ed.), 165-206, World Scientific, Singapore, 1990.

[4] Olshanetsky, M.A., Rogov, V.-B.K., Bound states in completely integrable systems with two types of particles, Ann. Inst. H. Poincaré, 29 (1978), 169-177.

[5] Olshanetsky, M.A., Perelomov, A.M., Classical integrable finite-dimensional systems related to Lie algebras, Phys. Reps., 71 (1981), 313-400.

[6] Ruijsenaars, S.N.M., Schneider, H., A new class of integrable systems and its relation to solitons, Ann. Phys. (NY), 170 (1986), 370-405.

[7] Calogero, F., A sequence of Lax matrices for certain integrable Hamiltonian systems, Lett. Nuovo Cim., 16 (1976), 22-24.

[ 8 ] Nicolaenko, B., Foias, C., Temam, R. (eds.), The connection between infinite dimensional 
and finite dimensional dynamical systems, Contemp. Math. 99, Amer. Math. Soc., Providence, 1987.

[9] Tanaka, S., On the $N$-tuple wave solutions of the Korteweg-de Vries equation, Publ. RIMS, Kyoto Univ., 8 (1972/73), 419-427.

[10] Ohmiya, M., On the generalized soliton solutions of the modified Korteweg-de Vries equation, Osaka J. Math., 11 (1974), 61-71.

[11] Ablowitz, M.J., Segur, H., Solitons and the Inverse Scattering Transform, SIAM Stud. Appl. Math., 4, SIAM, Philadelphia, 1981.

[12] Faddeev,L.D., Takhtajan, L.A., Hamiltonian methods in the theory of solitons, Springer, Berlin, Heidelberg, New York, 1987.

[13] Pöppe, C., Multipole solutions of the sine-Gordon equation, Heidelberg preprint, 1980.

[14] Tanaka, S., Non-linear Schrödinger equation and modified Korteweg-de Vries equation; construction of solutions in terms of scatering data, Publ. RIMS, Kyoto Univ., 10 (1975), 329-357.

[15] Wadati, M., Ohkuma, K., Multiple-pole solutions of the modified Korteweg-de Vries equation, J. Phys. Soc. Japan, 51 (1982), 2029-2035.

[16] Blaszak, M., On interacting solitons, Acta Phys. Polonica, A74 (1988), 439-444.

[17] Pogrebkov, A.K., Polivanov, M.K., The Liouville and sinh-Gordon equations. Singular solutions, dynamics of singularities and the inverse problem method, Sov. Sci. Rev. C Math. Phys, 5 (1985), 197-272.

[18] Guillemin, V., Sternberg, S., Symplectic techniques in physics, Cambridge University Press, Cambridge, 1984.

[19] Abraham, R., Marsden, J.E., Foundations of mechanics, Reading, Mass., Benjamin/ Cummings, 1978.

[20] Pogrebkov, A.K., Private communication.

[21] Gohberg, I., Lancaster, P., Rodman, L., Matrices and indefinite scalar products, Operator theory: advances and applications, 8, Birkhäuser, Basel, 1983.

[22] Ruijsenaars, S.N.M., Action-angle maps and scattering theory for some finite-dimensional integrable systems. III. Sutherland type systems and their duals, to appear in Publ. RIMS. Kyoto Univ., 31 (1995), no.2. 